\title{
Microwave-Assisted Branching Cascades: A Route to Diverse 3,4-Dihydroquinazolinone- Embedded Polyheterocyclic Scaffolds
}

\author{
Rajiv T. Sawant ${ }^{a}$, Marc Y. Stevens ${ }^{a}$ Christian Sköld $^{a}$ and Luke R. Odell*a \\ ${ }^{a}$ Organic Pharmaceutical Chemistry, Department of Medicinal Chemistry, Uppsala \\ Biomedical Center, Uppsala University, P.O. Box 574, SE-751 23 Uppsala, Sweden
}

luke.odell@orgfarm.uu.se

SUPPORTING INFORMATION 


\section{Contents}

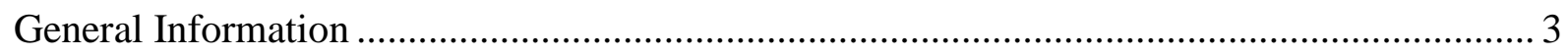

Optimization of reaction parameters for formation of 16a from $1 \mathbf{a}$ and $\mathbf{2 a}$............................ 4

General procedure A for preparation of 3,4-dihydroquinazolinones 16a-16h, 17-19, 20a-20b, 21a-21c, 22-24, 27a-27b, 28a-28c, 29a-29b, 30a-30b, 31a-31c, 32a/b/c-33a/b/c, 34a-34b, 36a-36b, exemplified by 2-Bromo-11,12-dimethoxy-5,8,9,13b-tetrahydro- $6 \mathrm{H}$ -

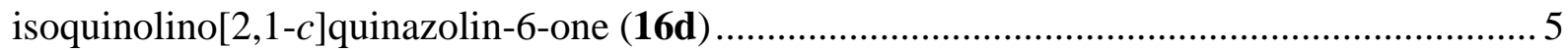

General procedure B for preparation of 3,4-dihydroquinazolinones 17, 23, 25, 35a, 35b, exemplified by 11-chloro-8,9,14,14b-tetrahydroindolo[2',3':3,4]pyrido[1,2-c]quinazolin6(5H)-one (23). 5

General procedure $C$ for preparation of pyridoquinazolinone methyl acetates 26a, 26a', 26b and $\mathbf{2 6} \mathbf{b}^{\prime}$, exemplified by $\mathbf{2 6 a} / \mathbf{2 6 a}$ '

General procedure $\mathrm{D}$ for one-pot, two-step preparation of C4-substituted 3,4dihydroquinazolinones 37a-37c, exemplified by 3-(3,4-dimethoxyphenethyl)-4-(nitromethyl)-

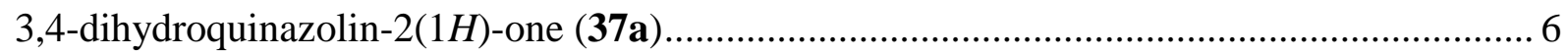

General procedure $\mathrm{E}$ for one-pot, two-step preparation of C4-substituted 3,4dihydroquinazolinones $38 \mathrm{a}-38 \mathrm{j}, 39 \mathrm{a}-39 \mathrm{~g}, 40 \mathrm{a}-40 \mathrm{c}$ and $41 \mathrm{a}-41 \mathrm{c}$, exemplified by $4-(1 \mathrm{H}$-Indol-

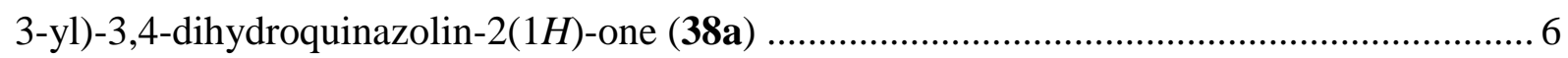

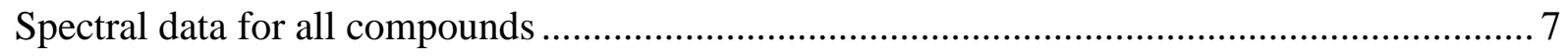

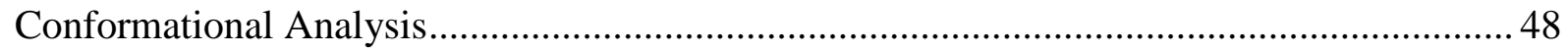

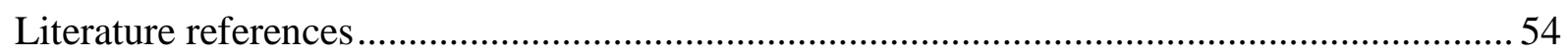

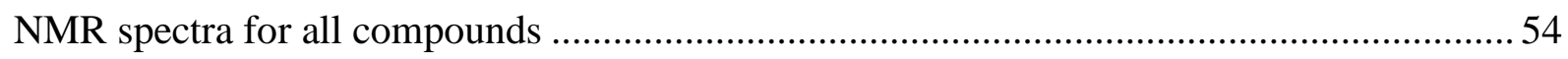




\section{General Information}

All reagents were purchased at the highest commercial quality and used without further purification. Yields refer to isolated, homogenous and spectroscopically pure material, unless otherwise stated. Reaction outcome was determined using EI-MS. Crude reaction mixtures were purified by silica gel chromatography (E. Merck silica gel, particle size 0.043-0.063 $\mathrm{mm})$. Thin layer chromatography was carried out using E. Merck silica plates (60F-254) with UV light $(254 \mathrm{~nm})$ and/or iodine vapor as the visualization agent. Preparative thin-layer chromatography was carried out using $2 \mathrm{~mm}$ thick E. Merck glass-backed silica plates (60F254). Microwave reactions were carried out in an Initiator single-mode reactor producing controlled radiation at $2450 \mathrm{MHz}$, and temperature was monitored via the built-in online IR sensor. ${ }^{1} \mathrm{H}$ NMR spectra were recorded at $400 \mathrm{MHz}$ and ${ }^{13} \mathrm{C}\left\{{ }^{1} \mathrm{H}\right\}$ NMRspectra at $100 \mathrm{MHz}$. The chemical shifts for ${ }^{1} \mathrm{H}$ NMR and ${ }^{13} \mathrm{C}\left\{{ }^{1} \mathrm{H}\right\}$ NMR spectra were referenced to tetramethylsilane via residual solvent signals $\left({ }^{1} \mathrm{H}, \mathrm{CDCl}_{3}\right.$ at $7.26 \mathrm{ppm}$, DMSO- $d_{6}$ at $2.50 \mathrm{ppm}$; ${ }^{13} \mathrm{C}, \mathrm{CDCl}_{3}$ at $77.16 \mathrm{ppm}$, DMSO- $d_{6}$ at $39.5 \mathrm{ppm}$ ). LC/MS was performed on an instrument equipped with a C18 column $(50 \times 3.0 \mathrm{~mm}$, particle size $2.6 \mu \mathrm{m}$, pore size $100 \AA)$. Accurate mass values were determined on a mass spectrometer equipped with an electrospray ion source and TOF detector. Electrophilic precursors $\mathbf{1 a}-\mathbf{1} \mathbf{j}$ were prepared following the literature procedure, ${ }^{[1]}$ and amines or carbon nucleophiles 2a-15e were purchased from commercial sources. 
Optimization of reaction parameters for formation of $16 a$ from $1 \mathrm{a}$ and $2 \mathrm{a}$ Table S1: Optimization of reaction parameters ${ }^{[\mathrm{a}]}$

\begin{tabular}{|c|c|c|c|c|c|}
\hline Entry & Acid (equiv.) & Solvent & $\begin{array}{c}\text { Temp } \\
\left({ }^{\circ} \mathrm{C}\right)\end{array}$ & $\begin{array}{l}\text { Time } \\
(\min )\end{array}$ & $\begin{array}{c}\text { Yield } \\
(\mathbf{1 6 a})(\%)\end{array}$ \\
\hline 1 & - & $\mathrm{EtOH}$ & 120 & 20 & - \\
\hline 2 & $\mathrm{AcOH}(10)$ & $\mathrm{EtOH}$ & 120 & 20 & - \\
\hline 3 & $\mathrm{HCl}(10)$ & $\mathrm{EtOH}$ & 130 & 20 & - \\
\hline 4 & $\begin{array}{c}\mathrm{Sc}(\mathrm{OTf})_{3} \\
\quad(0.1)\end{array}$ & $\mathrm{EtOH}$ & 120 & 20 & - \\
\hline 5 & $\begin{array}{c}\mathrm{Sc}(\mathrm{OTf})_{3} \\
\quad(0.1)\end{array}$ & $\mathrm{CH}_{2} \mathrm{Cl}_{2}$ & 100 & 20 & - \\
\hline 6 & $\begin{array}{c}\mathrm{HCO}_{2} \mathrm{H} \\
\text { (neat) }\end{array}$ & - & 130 & 20 & 36 \\
\hline 7 & $\mathrm{AcOH}$ (neat) & - & 130 & 10 & 88 \\
\hline 8 & $\mathrm{AcOH}$ (neat) & - & 120 & 20 & 88 \\
\hline 9 & $\mathrm{AcOH}$ (neat) & - & 100 & 20 & 84 \\
\hline 10 & AcOH (neat) & - & 140 & 20 & 95 \\
\hline 11 & AcOH (neat) & - & 140 & 10 & 93 \\
\hline
\end{tabular}

[a] Isolated yields. All reactions carried out on $0.22 \mathrm{mmol}$ scale. 
General procedure A for preparation of 3,4-dihydroquinazolinones 16a16h, 17-19, 20a-20b, 21a-21c, 22-24, 27a-27b, 28a-28c, 29a-29b, 30a-30b, 31a-31c, 32a/b/c-33a/b/c, 34a-34b, 36a-36b, exemplified by 2-Bromo-11,12dimethoxy-5,8,9,13b-tetrahydro-6H-isoquinolino[2,1-c]quinazolin-6-one (16d)

A $0.5-2 \mathrm{~mL}$ Pyrex process vial equipped with a stirring bar was charged with aldehyde 1a (40 $\mathrm{mg}, 155 \mu \mathrm{mol})$, amine $2 \mathrm{a}(37 \mathrm{mg}, 204 \mu \mathrm{mol})$ and $\mathrm{AcOH}(1 \mathrm{~mL})$. The vial was capped and subjected to microwave irradiation at $140{ }^{\circ} \mathrm{C}$ for $20 \mathrm{~min}$. After cooling to ambient temperature, the reaction mixture was concentrated in vacuo. Silica gel chromatography (30$100 \%$ EtOAc in $n$-pentane) provided the title compound as a white solid (56 mg, $144 \mu \mathrm{mol}$, $93 \%)$.

General procedure B for preparation of 3,4-dihydroquinazolinones 17, 23, 25, 35a, 35b, exemplified by 11-chloro-8,9,14,14btetrahydroindolo[2',3':3,4]pyrido[1,2-c]quinazolin-6(5H)-one (23)

Amine 3c $(34 \mathrm{mg}, 147 \mu \mathrm{mol}$, as the hydrochloride salt) was dissolved in EtOH $(1 \mathrm{~mL})$ at ambient temperature. $\mathrm{NaOH}(6 \mathrm{mg}, 150 \mu \mathrm{mol})$ was added, and the resulting mixture was stirred vigorously for $10 \mathrm{~min}$. The volatiles were evaporated and the residue was dissolved in acetic acid and transferred to a Pyrex process vial. Thereafter, aldehyde 1a (20 mg, $112 \mu \mathrm{mol})$ was added and general procedure $\mathbf{A}$ was followed.

General procedure $C$ for preparation of pyridoquinazolinone methyl acetates 26a, 26a', 26b and 26b', exemplified by 26a/26a'

A $0.5-2 \mathrm{~mL}$ Pyrex process vial was charged with aldehyde $\mathbf{1 a}(40 \mathrm{mg}, 224 \mu \mathrm{mol})$, amine $\mathbf{3 f}$ (55 mg, $289 \mu \mathrm{mol})$ and $\mathrm{AcOH}(1 \mathrm{~mL})$ and the resulting mixture was subjected to microwave irradiation at $140{ }^{\circ} \mathrm{C}$ for $1 \mathrm{~h}$. The reaction mixture was concentrated in vacuo and thereafter the residue was partitioned between $\mathrm{NaHCO}_{3}$ (sat., $4 \mathrm{~mL}$ ) and EtOAc $(20 \mathrm{~mL}$ ). The aqueous phase was extracted into EtOAc $(2 \times 20 \mathrm{~mL})$ and the combined organics were washed with brine, dried over $\mathrm{Na}_{2} \mathrm{SO}_{4}$ and concentrated in vacuo. The residue was used without purification in the next step. ESI $+=m / z 320.17$. 
To a stirred solution of the above residue in anhydrous dichloromethane at ambient temperature was added acetic anhydride $(25 \mathrm{mg}, 245 \mu \mathrm{mol})$ and DMAP (29 mg, $237 \mu \mathrm{mol})$. The resulting mixture was stirred for $1 \mathrm{~h}$, until full consumption of the alcohol was confirmed (LC/MS). The reaction mixture was concentrated under reduced pressure and the residue was purified by silica gel chromatography (35-50\% EtOAc in $n$-pentane), to give two diastereomers, syn (26a) and anti (26a').

\section{General procedure D for one-pot, two-step preparation of C4-substituted} 3,4-dihydroquinazolinones 37a-37c, exemplified by 3-(3,4dimethoxyphenethyl)-4-(nitromethyl)-3,4-dihydroquinazolin-2(1H)-one

A $0.5-2 \mathrm{~mL}$ Pyrex process vial was charged with aldehyde $\mathbf{1 a}(20 \mathrm{mg}, 112 \mu \mathrm{mol})$, amine $\mathbf{2 a}$ (26 mg, $181 \mu \mathrm{mol})$ and ethanol/acetic acid $(9: 1,1 \mathrm{~mL})$. The vial was sealed and subjected to microwave irradiation at $130{ }^{\circ} \mathrm{C}$ for $10 \mathrm{~min}$, after which nitromethane (13a, $\left.20 \mathrm{mg}, 328 \mu \mathrm{mol}\right)$ was added through the septum. The vial was heated by microwave at $130{ }^{\circ} \mathrm{C}$ for $10 \mathrm{~min}$, and thereafter the reaction mixture was concentrated in vacuo. Silica gel chromatography (55\% EtOAc in $n$-pentane) provided the title compound as a white solid (38 $\mathrm{mg}, 91 \%$ ).

General procedure $\mathrm{E}$ for one-pot, two-step preparation of $\mathrm{C} 4$-substituted 3,4-dihydroquinazolinones 38a-38j, 39a-39g, 40a-40c and 41a-41c, exemplified by 4-(1H-Indol-3-yl)-3,4-dihydroquinazolin-2(1H)-one (38a)

A $0.5-2 \mathrm{~mL}$ Pyrex process vial was charged with aldehyde $\mathbf{1 a}(40 \mathrm{mg}, 224 \mu \mathrm{mol}), \mathrm{NH}_{4} \mathrm{OAc}$ 15a $(34 \mathrm{mg}, 441 \mu \mathrm{mol})$ and acetic acid $(1 \mathrm{~mL})$. The vial was sealed and subjected to microwave irradiation at $130{ }^{\circ} \mathrm{C}$ for $10 \mathrm{~min}$, after which indole $(\mathbf{1 4 a}, 38 \mathrm{mg}, 290 \mu \mathrm{mol})$. The vial was re-sealed and heated by microwave at $130{ }^{\circ} \mathrm{C}$ for $20 \mathrm{~min}$, and thereafter the reaction mixture was concentrated in vacuo. Silica gel chromatography $(2-5 \% \mathrm{MeOH}$ in DCM or 30$85 \%$ EtOAc in $n$-pentane) provided the title compound as a white solid (56 mg, $212 \mu \mathrm{mol}$, $95 \%)$. 


\section{Spectral data for all compounds}

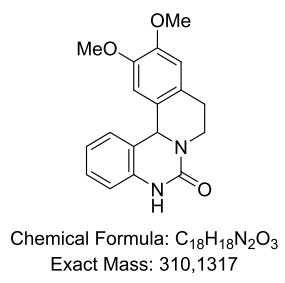

11,12-dimethoxy-5,8,9,13b-tetrahydro- $6 H$-isoquinolino[2,1-c]quinazolin-6-one 56162-13-1) (16a) ${ }^{[2]}$

Prepared following the general procedure (A), starting from aldehyde $\mathbf{1 a}$ (40 mg, $224 \mu \mathrm{mol}$ ) and $\mathbf{2 a}(52 \mathrm{mg}, 284 \mu \mathrm{mol})$.

Physical state and yield: white solid (65 mg, $209 \mu \mathrm{mol}, 95 \%)$

${ }^{1}$ H NMR (400 MHz, CDCl3): $\delta 8.36(\mathrm{~s}, 1 \mathrm{H}), 7.36-7.21(\mathrm{~m}, 1 \mathrm{H}), 7.20-7.12(\mathrm{~m}, 1 \mathrm{H}), 7.07$ $6.98(\mathrm{~m}, 1 \mathrm{H}), 6.89-6.79(\mathrm{~m}, 1 \mathrm{H}), 6.66(\mathrm{~s}, 1 \mathrm{H}), 6.53(\mathrm{~s}, 1 \mathrm{H}), 5.56(\mathrm{~s}, 1 \mathrm{H}), 4.72-4.28(\mathrm{~m}, 1 \mathrm{H})$, $3.85(\mathrm{~s}, 3 \mathrm{H}), 3.71(\mathrm{~s}, 3 \mathrm{H}), 3.32-3.02(\mathrm{~m}, 2 \mathrm{H}), 2.71(\mathrm{~d}, J=15.2 \mathrm{~Hz}, 1 \mathrm{H})$.

${ }^{13}$ C NMR (100 MHz, CDCl3): $\delta$ 155.4, 148.3, 147.1, 137.2, 128.6, 127.6, 127.0, 126.7, $121.6,120.0,114.4,112.1,109.4,57.6,55.9,42.0,27.0$.

Accurate mass (ESI, $\boldsymbol{m} / z)$ : calc' $d$ for $\mathrm{C}_{18} \mathrm{H}_{19} \mathrm{~N}_{2} \mathrm{O}_{3}\left(\left[\mathrm{M}+\mathrm{H}^{+}\right]\right): 311.1396$, found m/z 311.1393.

TLC (SiO $): R_{\mathrm{f}}=0.21$ (50\% EtOAc in $n$-pentane).

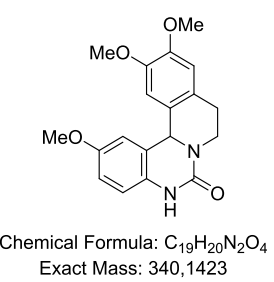

2,11,12-Trimethoxy-5,8,9,13b-tetrahydro-6H-isoquinolino[2,1-c]quinazolin-6-one (16b) Prepared following the general procedure (A), starting from aldehyde $\mathbf{1 b}$ (40 mg, $191 \mu \mathrm{mol}$ ) and $2 \mathbf{a}(45 \mathrm{mg}, 248 \mu \mathrm{mol})$.

Physical state and yield: white solid (61 mg, $179 \mu \mathrm{mol}, 94 \%)$ 
${ }^{1}$ H NMR (400 MHz, CDCl $): \delta 7.77$ (s, 1H), 6.79 (dd, J = 8.6, $\left.2.6 \mathrm{~Hz}, 1 \mathrm{H}\right), 6.74(\mathrm{~s}, 1 \mathrm{H})$, 6.73-6.71 (m, 1H), $6.65(\mathrm{~s}, 1 \mathrm{H}), 6.57(\mathrm{~s}, 1 \mathrm{H}), 5.51(\mathrm{~s}, 1 \mathrm{H}), 4.69-4.37(\mathrm{~m}, 1 \mathrm{H}), 3.85(\mathrm{~s}, 3 \mathrm{H})$, $3.76(\mathrm{~s}, 3 \mathrm{H}), 3.73(\mathrm{~s}, 3 \mathrm{H}), 3.35-2.99(\mathrm{~m}, 2 \mathrm{H}), 2.85-2.56(\mathrm{~m}, 1 \mathrm{H})$.

${ }^{13}$ C NMR (100 MHz, CDCl 3$): \delta 155.5,154.7,148.3,147.1,130.9,127.8,126.5,121.5$, $115.0,113.8,112.6,112.2,109.5,57.6,56.0,55.9,55.8,41.9,27.1$.

Accurate mass (ESI, $\boldsymbol{m} / z)$ : calc' $d$ for $\mathrm{C}_{19} \mathrm{H}_{19} \mathrm{~N}_{2} \mathrm{O}_{4}$ ([M- $\left.\left.\mathrm{H}^{+}\right]\right)$: 339.1345, found m/z 339.1355.

TLC $\left(\mathbf{S i O}_{2}\right): R_{\mathrm{f}}=0.21(50 \%$ EtOAc in $n$-pentane $)$

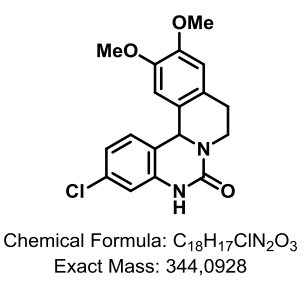

3-Chloro-11,12-dimethoxy-5,8,9,13b-tetrahydro-6H-isoquinolino[2,1-c]quinazolin-6-one (16c)

Prepared following the general procedure (A) starting from aldehyde 1c (40 mg, $187 \mu \mathrm{mol})$ and amine $\mathbf{2 a}(44 \mathrm{mg}, 243 \mu \mathrm{mol})$.

Physical state and yield: white solid (62 mg, $180 \mu \mathrm{mol}, 96 \%)$.

${ }^{1}$ H NMR (400 MHz, DMSO-d $): \delta 9.63(\mathrm{~s}, 1 \mathrm{H}), 7.41(\mathrm{~d}, J=8.1 \mathrm{~Hz}, 1 \mathrm{H}), 7.10(\mathrm{dd}, J=8.1$, $2.1 \mathrm{~Hz}, 1 \mathrm{H}), 6.92(\mathrm{~d}, J=2.1 \mathrm{~Hz}, 1 \mathrm{H}), 6.82(\mathrm{~s}, 1 \mathrm{H}), 6.44(\mathrm{~s}, 1 \mathrm{H}), 5.68(\mathrm{~s}, 1 \mathrm{H}), 4.31(\mathrm{ddd}, J=$ 12.9, 6.6, $2.0 \mathrm{~Hz}, 1 \mathrm{H}), 3.76(\mathrm{~s}, 3 \mathrm{H}), 3.60(\mathrm{~s}, 3 \mathrm{H}), 3.22(\mathrm{ddd}, J=12.9,11.3,5.0 \mathrm{~Hz}, 1 \mathrm{H}), 3.05$ (ddd, $J=17.4,11.2,6.6 \mathrm{~Hz}, 1 \mathrm{H}), 2.74-2.65(\mathrm{~m}, 1 \mathrm{H})$.

${ }^{13}$ C NMR (100 MHz, DMSO-d6): $\delta$ 153.8, 148.1, 146.6, 138.9, 132.7, 129.2, 127.4, 127.2, $120.6,118.6,113.3,112.8,109.2,56.4,55.2,55.1,41.6,25.6$.

Accurate mass (ESI, $\boldsymbol{m} / \boldsymbol{z}$ ): calc' $\mathrm{d}$ for $\mathrm{C}_{20} \mathrm{H}_{21} \mathrm{ClN}_{3} \mathrm{O}_{3}\left(\left[\mathrm{M}+\mathrm{MeCN}+\mathrm{H}^{+}\right]\right): 386.1271$, found $\mathrm{m} / \mathrm{z}$ 386.1281. 
TLC (SiO $): R_{\mathrm{f}}=0.13(50 \%$ EtOAc in $n$-pentane)

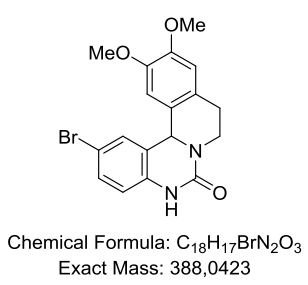

\section{2-Bromo-11,12-dimethoxy-5,8,9,13b-tetrahydro- $6 H$-isoquinolino[2,1-c]quinazolin-6-one} (16d)

Prepared following the general procedure (A) starting from aldehyde 1d (40 mg, $155 \mu \mathrm{mol}$ ) and amine $\mathbf{2 a}(44 \mathrm{mg}, 243 \mu \mathrm{mol})$.

Physical state and yield: white solid (56 mg, $143 \mu \mathrm{mol}, 93 \%)$.

${ }^{1}$ H NMR (400 MHz, DMSO-d d $_{\text {) }} \delta 9.54(\mathrm{~s}, 1 \mathrm{H}), 7.47$ (d, $\left.J=2.2 \mathrm{~Hz}, 1 \mathrm{H}\right), 7.37(\mathrm{dd}, J=8.5$, $2.2 \mathrm{~Hz}, 1 \mathrm{H}), 6.83-6.69(\mathrm{~m}, 2 \mathrm{H}), 6.39(\mathrm{~s}, 1 \mathrm{H}), 5.57(\mathrm{~s}, 1 \mathrm{H}), 4.32-4.14(\mathrm{~m}, 1 \mathrm{H}), 3.68(\mathrm{~s}, 3 \mathrm{H})$, $3.53(\mathrm{~s}, 3 \mathrm{H}), 3.17-3.04(\mathrm{~m}, 1 \mathrm{H}), 3.02-2.87(\mathrm{~m}, 1 \mathrm{H}), 2.61(\mathrm{dd}, J=16.2,3.5 \mathrm{~Hz}, 1 \mathrm{H})$.

${ }^{13}$ C NMR (100 MHz, DMSO-d6): $\delta$ 154.0, 148.2, 146.6, 136.9, 131.2, 129.7, 127.4, 127.0, 122.2, 115.9, 112.8, 112.2, 109.5, 56.3, 55.6, 55.6, 41.5, 25.7.

Accurate mass (ESI, $\boldsymbol{m} / \boldsymbol{z}$ ): calc'd for $\mathrm{C}_{20} \mathrm{H}_{21} \mathrm{BrN}_{3} \mathrm{O}_{3}\left([\mathrm{M}+\mathrm{MeCN}+\mathrm{H}]^{+}\right): 430.0766$, found $\mathrm{m} / \mathrm{z}$ 430.0782 .

TLC (SiO2): $R_{\mathrm{f}}=0.13(50 \%$ EtOAc in $n$-pentane $)$.

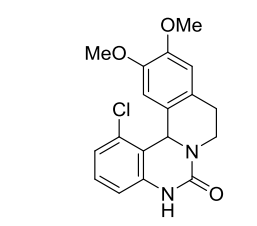

Chemical Formula: $\mathrm{C}_{18} \mathrm{H}_{17} \mathrm{ClN}_{2} \mathrm{O}_{3}$ Exact Mass: 344,0928 


\section{1-Chloro-11,12-dimethoxy-5,8,9,13b-tetrahydro-6H-isoquinolino[2,1-c]quinazolin-6-one}

(16e)

Prepared following the general procedure (A) starting from aldehyde 1e $(41 \mathrm{mg}, 193 \mu \mathrm{mol})$ and amine $\mathbf{2 a}(46 \mathrm{mg}, 247 \mu \mathrm{mol})$.

Physical state and yield: white solid (56 mg, $155 \mu \mathrm{mol}, 81 \%)$.

${ }^{1}$ H NMR (400 MHz, CDCl 3$): \delta 9.21(\mathrm{~s}, 1 \mathrm{H}), 6.73(\mathrm{~d}, J=7.9 \mathrm{~Hz}, 1 \mathrm{H}), 6.59(\mathrm{~s}, 1 \mathrm{H}), 6.31(\mathrm{~s}$, $1 \mathrm{H}), 5.80(\mathrm{~s}, 1 \mathrm{H}), 4.70-4.40(\mathrm{~m}, 1 \mathrm{H}), 3.78(\mathrm{~s}, 3 \mathrm{H}), 3.62(\mathrm{~s}, 3 \mathrm{H}), 3.50-3.12(\mathrm{~m}, 2 \mathrm{H}), 2.95-2.46$ $(\mathrm{m}, 1 \mathrm{H})$.

${ }^{13}$ C NMR (100 MHz, CDCl 3$): \delta 155.3,148.4,146.9,138.5,132.6,129.7,127.3,127.0$, $122.4,117.7,113.2,112.2,108.7,56.3,55.9,55.8,42.9,25.6$.

Accurate mass $($ ESI, $\boldsymbol{m} / \boldsymbol{z})$ : calc' $\mathrm{d}$ for $\mathrm{C}_{20} \mathrm{H}_{21} \mathrm{ClN}_{3} \mathrm{O}_{3}\left([\mathrm{M}+\mathrm{MeCN}+\mathrm{H}]^{+}\right): 386.1271$, found $\mathrm{m} / \mathrm{z}$ 386.1279 .

TLC (SiO2): $R_{\mathrm{f}}=0.35$ (30\% EtOAc in $n$-pentane).

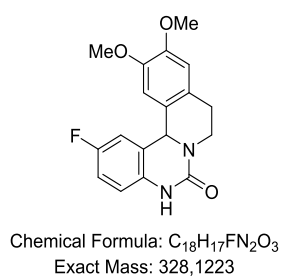

\section{2-Fluoro-11,12-dimethoxy-5,8,9,13b-tetrahydro-6H-isoquinolino[2,1-c]quinazolin-6-one} (16f)

Prepared following the general procedure (A) starting from aldehyde 1f (30 mg, $155 \mu \mathrm{mol})$ and amine $2 \mathbf{a}(42 \mathrm{mg}, 224 \mu \mathrm{mol})$.

Physical state and yield: white solid (45 mg, $137 \mu \mathrm{mol}, 89 \%)$. 
${ }^{1}$ H NMR (400 MHz, CDCl $): \delta 8.95(\mathrm{~s}, 1 \mathrm{H}), 6.95(\mathrm{td}, J=8.5,2.8 \mathrm{~Hz}, 1 \mathrm{H}), 6.89-6.79(\mathrm{~m}$, 2H), $6.67(\mathrm{~s}, 1 \mathrm{H}), 6.54(\mathrm{~s}, 1 \mathrm{H}), 5.52(\mathrm{~s}, 1 \mathrm{H}), 4.52(\mathrm{ddd}, J=12.1,5.6,2.4 \mathrm{~Hz}, 1 \mathrm{H}), 3.86(\mathrm{~s}$, 3H), $3.76(\mathrm{~s}, 3 \mathrm{H}), 3.25-3.16(\mathrm{~m}, 1 \mathrm{H}), 3.16-3.05(\mathrm{~m}, 1 \mathrm{H}), 2.75-2.66(\mathrm{~m}, 1 \mathrm{H})$.

${ }^{13}$ C NMR (100 MHz, CDCl $): \delta 157.9\left(\mathrm{~d},{ }^{1} J_{\mathrm{CF}}=240.1 \mathrm{~Hz}\right), 155.8,148.4,147.2,133.7(\mathrm{~d}$, $\left.{ }^{4} J_{\mathrm{CF}}=2.2 \mathrm{~Hz}\right), 127.8,125.8,121.9\left(\mathrm{~d},{ }^{3} J_{\mathrm{CF}}=7.3 \mathrm{~Hz}\right), 115.4\left(\mathrm{~d},{ }^{3} J_{\mathrm{CF}}=7.9 \mathrm{~Hz}\right), 115.2\left(\mathrm{~d},{ }^{2} J_{\mathrm{CF}}\right.$ $=22.9 \mathrm{~Hz}), 113.2\left(\mathrm{~d},{ }^{2} J_{\mathrm{CF}}=24.2 \mathrm{~Hz}\right), 112.2,109.4,57.1,57.1,56.0,55.9,41.7,27.2$.

Accurate mass (ESI, $\boldsymbol{m} / \boldsymbol{z})$ : calc'd for $\mathrm{C}_{20} \mathrm{H}_{21} \mathrm{FN}_{3} \mathrm{O}_{3}\left([\mathrm{M}+\mathrm{MeCN}+\mathrm{H}]^{+}\right): 370.1595$, found $\mathrm{m} / \mathrm{z}$ 370.1571.

TLC (SiO 2$): R_{\mathrm{f}}=0.1$ (30\% EtOAc in $n$-pentane).

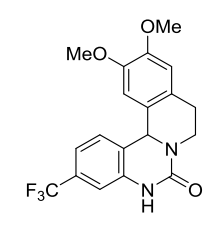

Chemical Formula: $\mathrm{C}_{19} \mathrm{H}_{17} \mathrm{~F}_{3} \mathrm{~N}_{2} \mathrm{O}_{3}$ Exact Mass: 378,1191

11,12-Dimethoxy-3-(trifluoromethyl)-5,8,9,13b-tetrahydro-6H-isoquinolino[2,1c]quinazolin-6-one (16g)

Prepared following the general procedure (A) starting from aldehyde $1 \mathrm{~g}$ (40 mg, $162 \mu \mathrm{mol}$ ) and amine $\mathbf{2 a}(38 \mathrm{mg}, 210 \mu \mathrm{mol})$.

Physical state and yield: white solid (55 mg, $145 \mu \mathrm{mol}, 90 \%)$.

${ }^{1}$ H NMR (400 MHz, CDCl3): $\delta 9.79(\mathrm{~s}, 1 \mathrm{H}), 7.60(\mathrm{~d}, J=8.0 \mathrm{~Hz}, 1 \mathrm{H}), 7.40(\mathrm{dt}, J=8.0,1.8$, $0.8 \mathrm{~Hz}, 1 \mathrm{H}), 7.20(\mathrm{~d}, J=1.8 \mathrm{~Hz}, 1 \mathrm{H}), 6.85(\mathrm{~s}, 1 \mathrm{H}), 6.46(\mathrm{~s}, 1 \mathrm{H}), 5.78(\mathrm{~s}, 1 \mathrm{H}), 4.32$ (ddd, $J=$ 12.9, 6.6, $2.0 \mathrm{~Hz}, 1 \mathrm{H}), 3.77$ (s, 3H), 3.60 (s, 3H), 3.23 (ddd, $J=12.8,11.4,4.9 \mathrm{~Hz}, 1 \mathrm{H}), 3.05$ (ddd, $J=17.4,11.4,6.5 \mathrm{~Hz}, 1 \mathrm{H}), 2.77-2.68(\mathrm{~m}, 1 \mathrm{H})$. 
${ }^{13}$ C NMR (100 MHz, CDCl $): \delta 153.4,147.8,146.2,137.9,128.7\left(q,{ }^{2} J_{\mathrm{CF}}=31.8 \mathrm{~Hz}\right), 128.0$, $127.0,126.2,123.61\left(\mathrm{q},{ }^{1} J_{\mathrm{CF}}=272.1 \mathrm{~Hz}\right), 123.59\left(\mathrm{q},{ }^{4} J_{\mathrm{CF}}=1.0 \mathrm{~Hz}\right), 117.00\left(\mathrm{q},{ }^{3} J_{\mathrm{CF}}=4.1 \mathrm{~Hz}\right)$, $112.5,109.7\left(\mathrm{q},{ }^{3} J_{\mathrm{CF}}=3.9 \mathrm{~Hz}\right), 109.0,56.1,55.2,55.1,41.1,25.3$.

Accurate mass (ESI, m/z): calc'd for $\mathrm{C}_{19} \mathrm{H}_{18} \mathrm{~F}_{3} \mathrm{~N}_{2} \mathrm{O}_{3}\left([\mathrm{M}+\mathrm{H}]^{+}\right)$: 379.1270, found $\mathrm{m} / \mathrm{z}$ 379.1274.

TLC (SiO) $): R_{\mathrm{f}}=0.15(60 \%$ EtOAc in $n$-pentane $)$.

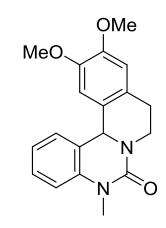

Chemical Formula: $\mathrm{C}_{19} \mathrm{H}_{20} \mathrm{~N}_{2} \mathrm{O}_{3}$

Exact Mass: 324,1474

11,12-Dimethoxy-5-methyl-5,8,9,13b-tetrahydro-6H-isoquinolino[2,1-c]quinazolin-6-one (16h)

Prepared following the general procedure (A) starting from aldehyde $\mathbf{1 h}$ (40 $\mathrm{mg}, 207 \mu \mathrm{mol})$ and amine $\mathbf{2 a}(49 \mathrm{mg}, 269 \mu \mathrm{mol})$.

Physical state and yield: white solid (62 mg, $191 \mu \mathrm{mol}, 92 \%)$.

${ }^{1}$ H NMR (400 MHz, DMSO-d6): $\delta$ 7.44-7.38 (m, 1H), 7.37-7.33 (m, 1H), 7.19-7.13 (m, 1H), 7.08-7.04 (m, 1H), $6.83(\mathrm{~s}, 1 \mathrm{H}), 6.51(\mathrm{~s}, 1 \mathrm{H}), 5.64(\mathrm{~s}, 1 \mathrm{H}), 4.40-4.21(\mathrm{~m}, 1 \mathrm{H}), 3.77(\mathrm{~s}$, $3 \mathrm{H}), 3.60(\mathrm{~s}, 3 \mathrm{H}), 3.29-3.21(\mathrm{~m}, 1 \mathrm{H}), 3.21(\mathrm{~s}, 3 \mathrm{H}), 3.09-2.98(\mathrm{~m}, 1 \mathrm{H}), 2.75-2.66(\mathrm{~m}, 1 \mathrm{H})$.

${ }^{13}$ C NMR (100 MHz, DMSO-d6): $\delta$ 155.7, 148.3, 147.1, 139.7, 128.5, 127.9, 126.21, 126.17, 122.9, 121.6, 113.0, 112.1, 109.7, 56.7, 56.0, 55.9, 42.7, 30.4, 27.1.

Accurate mass (ESI, $\boldsymbol{m} / z)$ : calc' $d$ for $\mathrm{C}_{19} \mathrm{H}_{21} \mathrm{~N}_{2} \mathrm{O}_{3}\left([\mathrm{M}+\mathrm{H}]^{+}\right)$: 325.1552 , found $m / z$ 325.1555.

TLC $\left(\mathrm{SiO}_{2}\right): R_{\mathrm{f}}=0.26(50 \%$ EtOAc in $n$-pentane) 


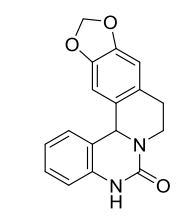

Chemical Formula: $\mathrm{C}_{17} \mathrm{H}_{14} \mathrm{~N}_{2} \mathrm{O}_{3}$ Exact Mass: 294,1004

5,8,9,14b-Tetrahydro-6H-[1,3]dioxolo[4',5':6,7]isoquinolino[2,1-c]quinazolin-6-one (17) Prepared following the general procedure (B) starting from aldehyde 1a (20 mg, $112 \mu \mathrm{mol})$ and amine $\mathbf{2 b}(29 \mathrm{mg}, 145 \mu \mathrm{mol})$.

Physical state and yield: white solid (27 mg, $92 \mu \mathrm{mol}, 82 \%)$. A yield of 54\% was obtained when using the hydrochloride salt of the amine directly following general procedure (A).

${ }^{1}$ H NMR (400 MHz, DMSO-d6): $\delta 9.42(\mathrm{~s}, 1 \mathrm{H}), 7.36-7.27(\mathrm{~m}, 1 \mathrm{H}), 7.24(\mathrm{td}, J=7.9,1.5 \mathrm{~Hz}$, 1H), $7.02(\mathrm{td}, J=7.5,1.2 \mathrm{~Hz}, 1 \mathrm{H}), 6.84(\mathrm{dd}, J=7.9,1.2 \mathrm{~Hz}, 1 \mathrm{H}), 6.76(\mathrm{~s}, 1 \mathrm{H}), 6.28(\mathrm{~s}, 1 \mathrm{H})$, $5.93(\mathrm{~d}, J=1.0 \mathrm{~Hz}, 1 \mathrm{H}), 5.88(\mathrm{~d}, J=1.0 \mathrm{~Hz}, 1 \mathrm{H}), 5.58(\mathrm{~s}, 1 \mathrm{H}), 4.22(\mathrm{ddd}, J=12.9,6.9,2.8$ $\mathrm{Hz}, 1 \mathrm{H}), 3.24-3.16(\mathrm{~m}, 1 \mathrm{H}), 3.02(\mathrm{ddd}, J=17.1,10.5,6.9 \mathrm{~Hz}, 1 \mathrm{H}), 2.67$ (ddd, $J=17.1,5.7$, $2.8 \mathrm{~Hz}, 1 \mathrm{H})$.

${ }^{13}$ C NMR (100 MHz, DMSO-d $): \delta$ 154.1, 146.2, 145.1, 137.3, 129.9, 128.5, 128.4, 127.7, 120.9, 119.1, 113.8, 109.1, 105.2, 100.8, 56.9, 41.5, 26.0.

Accurate mass (ESI, $\boldsymbol{m} / \mathbf{z})$ : calc'd for $\mathrm{C}_{19} \mathrm{H}_{18} \mathrm{~N}_{3} \mathrm{O}_{3}\left([\mathrm{M}+\mathrm{MeCN}+\mathrm{H}]^{+}\right)$: 336.1348 , found $\mathrm{m} / z$ 336.1363.

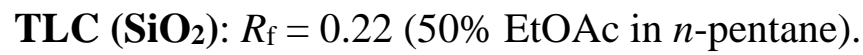




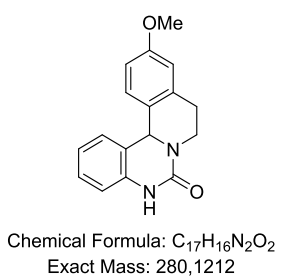

11-Methoxy-5,8,9,13b-tetrahydro-6H-isoquinolino[2,1-c]quinazolin-6-one (18)

Prepared following the general procedure (A) starting from aldehyde 1a (40 mg, $224 \mu \mathrm{mol}$ ) and amine $2 \mathrm{c}$ (44 mg, $290 \mu \mathrm{mol})$.

Physical state and yield: yellow solid (52 mg, $185 \mu \mathrm{mol}, 83 \%)$.

${ }^{1}$ H NMR (400 MHz, CDCl3): $\delta 8.04(\mathrm{~s}, 1 \mathrm{H}), 7.24(\mathrm{dd}, J=7.7,1.4 \mathrm{~Hz}, 1 \mathrm{H}), 7.15$ (ddd, $J=$ 7.7, 1.4, $0.7 \mathrm{~Hz}, 1 \mathrm{H}), 7.02(\mathrm{td}, J=7.5,1.1 \mathrm{~Hz}, 1 \mathrm{H}), 6.92(\mathrm{dd}, J=8.5 \mathrm{~Hz}, 1 \mathrm{H}), 6.80(\mathrm{dd}, J=$ 7.9, $1.1 \mathrm{~Hz}, 1 \mathrm{H}), 6.72(\mathrm{~d}, J=2.7 \mathrm{~Hz}, 1 \mathrm{H}), 6.68(\mathrm{dd}, J=8.5,2.7 \mathrm{~Hz}, 1 \mathrm{H}), 5.58(\mathrm{~s}, 1 \mathrm{H}), 4.58-$ $4.46(\mathrm{~m}, 1 \mathrm{H}), 3.78(\mathrm{~s}, 3 \mathrm{H}), 3.34-3.16(\mathrm{~m}, 2 \mathrm{H}), 2.86-2.74(\mathrm{~m}, 1 \mathrm{H})$.

${ }^{13}$ C NMR (100 MHz, CDCl3): $\delta$ 158.7, 155.2, 137.0, 136.8, 128.5, 127.6, 127.1, 126.9, $121.6,119.8,114.23,114.18,111.8,57.5,55.3,41.8,27.7$.

Accurate mass (ESI, $\boldsymbol{m} / \boldsymbol{z}$ ): calc'd for $\mathrm{C}_{17} \mathrm{H}_{17} \mathrm{~N}_{2} \mathrm{O}_{2}\left([\mathrm{M}+\mathrm{H}]^{+}\right)$: 281.1290, found $m / z$ 280.1280.

TLC (SiO2): $R_{\mathrm{f}}=0.23(50 \%$ EtOAc in $n$-pentane)

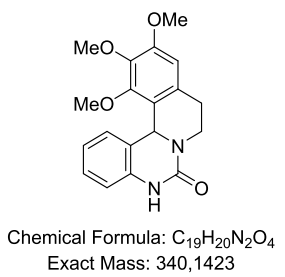

11,12,13-Trimethoxy-5,8,9,13b-tetrahydro-6H-isoquinolino[2,1-c]quinazolin-6-one (19). Prepared following the general procedure (A) but with 40 min of heating, starting from aldehyde 1a (20 mg, $112 \mu \mathrm{mol})$ and amine $\mathbf{2 d}(31 \mathrm{mg}, 145 \mu \mathrm{mol})$.

Physical state and yield: yellow solid (23 mg, $68 \mu \mathrm{mol}, 61 \%)$. 
${ }^{1}$ H NMR (400 MHz, DMSO-d $): \delta 9.47$ (s, 1H), 7.19-7.09 (m, 1H), 6.97-6.89 (m, 1H), 6.86-6.80 (m, 1H), $6.75(\mathrm{~s}, 1 \mathrm{H}), 6.51-6.47(\mathrm{~m}, 1 \mathrm{H}), 5.42(\mathrm{~s}, 1 \mathrm{H}), 4.20(\mathrm{ddd}, J=12.1,4.6,2.1$ $\mathrm{Hz}, 1 \mathrm{H}), 3.83(\mathrm{~s}, 3 \mathrm{H}), 3.81(\mathrm{~s}, 3 \mathrm{H}), 3.71(\mathrm{~s}, 3 \mathrm{H}), 2.85(\mathrm{td}, J=12.1,2.5 \mathrm{~Hz}, 1 \mathrm{H}), 2.71(\mathrm{dd}, J=$ $15.8,2.5 \mathrm{~Hz}, 1 \mathrm{H}), 2.60-2.53(\mathrm{~m}, 1 \mathrm{H})$.

${ }^{13}$ C NMR (100 MHz, DMSO-d6): $\delta$ 155.7, 152.5, 150.7, 139.7, 139.1, 132.1, 127.5, 124.1, $123.4,120.9,116.7,113.5,108.1,60.5,60.4,55.8,51.6,42.8,29.7$.

Accurate mass (ESI, $\boldsymbol{m} / z)$ : calc'd for $\mathrm{C}_{19} \mathrm{H}_{20} \mathrm{~N}_{2} \mathrm{O}_{4}\left([\mathrm{M}+\mathrm{H}]^{+}\right)$: 341.1496 , found $m / z$ 341.1490.

TLC (SiO2): $R_{\mathrm{f}}=0.22(50 \%$ EtOAc in $n$-pentane)

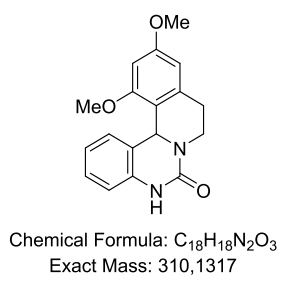

11,13-Dimethoxy-5,8,9,13b-tetrahydro-6H-isoquinolino[2,1-c]quinazolin-6-one (20a) Prepared following the general procedure (A) but with $40 \mathrm{~min}$ of heating, starting from aldehyde 1a (40 mg, $224 \mu \mathrm{mol})$ and amine $2 \mathbf{e}(44 \mathrm{mg}, 248 \mu \mathrm{mol})$.

Physical state and yield: yellow solid (66 mg, $214 \mu \mathrm{mol}, 96 \%)$.

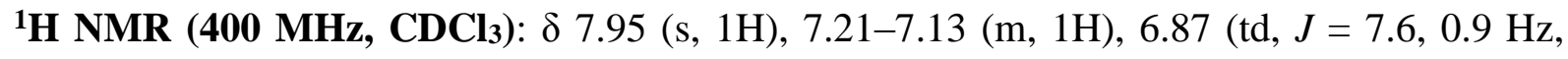
2H), 6.58-6.52 (m, 1H), $6.49(\mathrm{~d}, J=2.3 \mathrm{~Hz}, 1 \mathrm{H}), 6.40(\mathrm{~d}, J=2.3 \mathrm{~Hz}, 1 \mathrm{H}), 5.64(\mathrm{~s}, 1 \mathrm{H}), 4.44$ (ddd, $J=12.2,4.3,2.4 \mathrm{~Hz}, 1 \mathrm{H}), 3.86(\mathrm{~s}, 3 \mathrm{H}), 3.76(\mathrm{~s}, 3 \mathrm{H}), 3.05(\mathrm{td}, J=11.9,3.3 \mathrm{~Hz}, 1 \mathrm{H})$, $2.77(\mathrm{td}, J=4.3,0.8 \mathrm{~Hz}, 1 \mathrm{H}), 2.70(\mathrm{dt}, J=15.3,2.9 \mathrm{~Hz}, 1 \mathrm{H})$.

${ }^{13}$ C NMR (100 MHz, CDCl3): $\delta$ 160.1, 158.2, 157.1, 139.1, 138.8, 127.8, 124.4, 123.5, $121.9,113.8,112.7,105.1,96.9,55.5,55.4,51.7,39.8,30.9$.

Accurate mass (ESI, $\boldsymbol{m} / z)$ : calc'd for $\mathrm{C}_{18} \mathrm{H}_{19} \mathrm{~N}_{2} \mathrm{O}_{3}\left([\mathrm{M}+\mathrm{H}]^{+}\right): 311.1396$, found $m / z$ 311.1391. 
TLC (SiO $): R_{\mathrm{f}}=0.22(50 \%$ EtOAc in $n$-pentane).

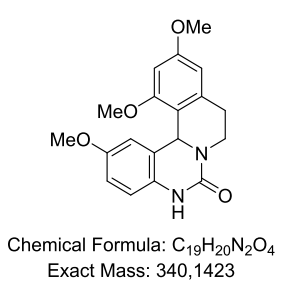

2,11,13-Trimethoxy-5,8,9,13b-tetrahydro-6H-isoquinolino[2,1-c]quinazolin-6-one (20b) Prepared following the general procedure (A) but with $40 \mathrm{~min}$ of heating, starting from aldehyde $\mathbf{1 b}$ (40 mg, $191 \mu \mathrm{mol})$ and amine $2 \mathbf{e}(45 \mathrm{mg}, 248 \mu \mathrm{mol})$.

Physical state and yield: white solid (61 mg, $179 \mu \mathrm{mol}, 94 \%)$.

${ }^{1}$ H NMR (400 MHz, CDCl3): $\delta 7.20(\mathrm{~s}, 1 \mathrm{H}), 6.84-6.69(\mathrm{~m}, 2 \mathrm{H}), 6.50(\mathrm{~d}, J=2.3 \mathrm{~Hz}, 1 \mathrm{H})$, $6.41(\mathrm{~d}, J=2.3 \mathrm{~Hz}, 1 \mathrm{H}), 6.25-6.06(\mathrm{~m}, 1 \mathrm{H}), 5.64(\mathrm{~s}, 1 \mathrm{H}), 4.48-4.29(\mathrm{~m}, 1 \mathrm{H}), 3.88(\mathrm{~s}, 3 \mathrm{H})$, $3.79(\mathrm{~s}, 3 \mathrm{H}), 3.68(\mathrm{~s}, 3 \mathrm{H}), 3.05(\mathrm{td}, J=11.8,3.4 \mathrm{~Hz}, 1 \mathrm{H}), 2.87-2.60(\mathrm{~m}, 2 \mathrm{H})$.

${ }^{13}$ C NMR (100 MHz, CDCl3): $\delta$ 160.1, 158.1, 157.2, 155.2, 139.2, 132.3, 125.2, 114.1, $112.5,112.4,111.2,105.2,96.9,55.8,55.6,55.4,51.8,39.9,30.9$.

Accurate mass (ESI, $\boldsymbol{m} / z)$ : calc'd for $\mathrm{C}_{21} \mathrm{H}_{24} \mathrm{~N}_{3} \mathrm{O}_{4}\left([\mathrm{M}+\mathrm{MeCN}+\mathrm{H}]^{+}\right)$: 382.1767 , found $\mathrm{m} / \mathrm{z}$ 382.1777 .

TLC (SiO2): $R_{\mathrm{f}}=0.22(50 \%$ EtOAc in $n$-pentane).

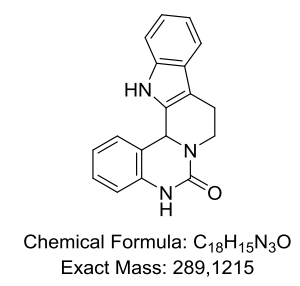


8,9,14,14b-Tetrahydroindolo[2',3':3,4]pyrido[1,2-c]quinazolin-6(5H)-one (21a).

Prepared following the general procedure (A) starting from aldehyde 1a (40 mg, $224 \mu \mathrm{mol}$ ) and amine 3a (46 mg, $287 \mu \mathrm{mol})$.

Physical state and yield: white solid (58 mg, $200 \mu \mathrm{mol}, 90 \%)$.

${ }^{1}$ H NMR (400 MHz, DMSO-d $)$ ): $\delta 10.39$ (s, 1H), 9.47 (s, 1H), 7.54-7.46 (m, 1H), 7.42-7.38 (m, 1H), 7.35-7.30 (m, 1H), 7.28-7.19 (m, 1H), 7.08-7.01 (m, 2H), 7.00-6.95 (m, 1H), 6.83$6.79(\mathrm{~m}, 1 \mathrm{H}), 6.02(\mathrm{~s}, 1 \mathrm{H}), 4.49(\mathrm{dd}, J=12.9,5.5 \mathrm{~Hz}, 1 \mathrm{H}), 3.21-3.06(\mathrm{~m}, 1 \mathrm{H}), 3.06-2.89(\mathrm{~m}$, $1 \mathrm{H}), 2.72-2.57(\mathrm{~m}, 1 \mathrm{H})$.

${ }^{13}$ C NMR (100 MHz, DMSO-d6): $\delta$ 154.0, 136.9, 136.0, 132.7, 128.7, 127.0, 126.8, 121.5, $121.1,118.7,117.8,117.6,113.5,111.6,107.8,55.3,42.4,19.4$.

Accurate mass (ESI, $\boldsymbol{m} / z)$ : calc'd for $\mathrm{C}_{18} \mathrm{H}_{16} \mathrm{~N}_{3} \mathrm{O}\left([\mathrm{M}+\mathrm{H}]^{+}\right)$: 290.1293, found $m / z$ 290.1287.

TLC (SiO2): $R_{\mathrm{f}}=0.22(50 \%$ EtOAc in $n$-pentane)

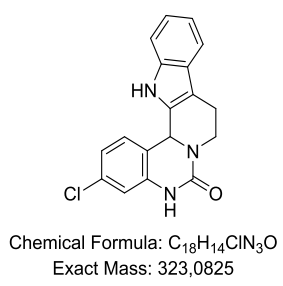

3-Chloro-8,9,14,14b-tetrahydroindolo[2',3':3,4]pyrido[1,2-c]quinazolin-6(5H)-one (21b) Prepared following the general procedure (A) starting from aldehyde $1 \mathbf{c}(20 \mathrm{mg}, 94 \mu \mathrm{mol})$ and amine 3a $(23 \mathrm{mg}, 143 \mu \mathrm{mol})$.

Physical state and yield: white solid (23 mg, $71 \mu \mathrm{mol}, 75 \%)$.

${ }^{1}$ H NMR (400 MHz, DMSO-d6): $\delta 10.43(\mathrm{~s}, 1 \mathrm{H}), 9.64(\mathrm{~s}, 1 \mathrm{H}), 7.52(\mathrm{~d}, J=8.2 \mathrm{~Hz}, 1 \mathrm{H})$, 7.57-7.48 (m, 1H), 7.35-7.29 (m, 1H), $7.10(\mathrm{dd}, J=8.2,2.1 \mathrm{~Hz}, 1 \mathrm{H}), 7.05(\mathrm{ddd}, J=8.2,7.0$, $1.3 \mathrm{~Hz}, 1 \mathrm{H}), 6.97$ (ddd, $J=8.0,7.0,1.1 \mathrm{~Hz}, 1 \mathrm{H}), 6.85(\mathrm{~d}, J=2.1 \mathrm{~Hz}, 1 \mathrm{H}), 6.15-5.76(\mathrm{~m}, 1 \mathrm{H})$, $4.48(\mathrm{dd}, J=13.0,5.6 \mathrm{~Hz}, 1 \mathrm{H}), 3.20-3.09(\mathrm{~m}, 1 \mathrm{H}), 3.03-2.90(\mathrm{~m}, 1 \mathrm{H}), 2.69-2.60(\mathrm{~m}, 1 \mathrm{H})$. 
${ }^{13}$ C NMR (100 MHz, DMSO-d $)$ : $\delta$ 153.6, 138.5, 136.0, 133.0, 132.3, 128.9, 126.7, 121.2, 121.1, 118.8, 117.7, 116.7, 112.9, 111.6, 107.9, 54.8, 42.5, 19.3.

Accurate mass (ESI, $\boldsymbol{m} / \boldsymbol{z})$ : calc'd for $\mathrm{C}_{20} \mathrm{H}_{18} \mathrm{ClN} \mathrm{N}_{4} \mathrm{O}\left([\mathrm{M}+\mathrm{MeCN}+\mathrm{H}]^{+}\right): 365.1169$, found $\mathrm{m} / \mathrm{z}$ 365.1183.

TLC $\left(\mathbf{S i O}_{2}\right): R_{\mathrm{f}}=0.21(40 \%$ EtOAc in $n$-pentane $)$.

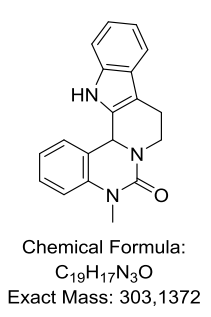

5-Methyl-8,9,14,14b-tetrahydroindolo[ $\left.2^{\prime}, 3^{\prime}: 3,4\right]$ pyrido[1,2-c]quinazolin-6(5H)-one (21c) Prepared following the general procedure (A) starting from aldehyde $\mathbf{1 h}$ (30 mg, $155 \mu \mathrm{mol})$ and amine $\mathbf{3 a}(32 \mathrm{mg}, 200 \mu \mathrm{mol})$.

Physical state and yield: red solid (44 mg, $145 \mu \mathrm{mol}, 93 \%)$.

${ }^{1}$ H NMR (400 MHz, DMSO-d $): \delta 10.58(\mathrm{~s}, 1 \mathrm{H}), 7.58-7.52(\mathrm{~m}, 1 \mathrm{H}), 7.49-7.40(\mathrm{~m}, 2 \mathrm{H})$, $7.38(\mathrm{~d}, J=8.0 \mathrm{~Hz}, 1 \mathrm{H}), 7.25-7.18(\mathrm{~m}, 1 \mathrm{H}), 7.12-7.07(\mathrm{~m}, 1 \mathrm{H}), 7.07-6.98(\mathrm{~m}, 2 \mathrm{H}), 5.99(\mathrm{~s}$, $1 \mathrm{H}), 4.52(\mathrm{dd}, J=12.9,5.5 \mathrm{~Hz}, 1 \mathrm{H}), 3.24(\mathrm{~s}, 4 \mathrm{H}), 3.12-2.93(\mathrm{~m}, 1 \mathrm{H}), 2.81-2.62(\mathrm{~m}, 1 \mathrm{H})$.

${ }^{13}$ C NMR (100 MHz, DMSO-d $): \delta$ 154.7, 138.4, 136.0, 132.3, 128.9, 126.7, 126.7, 122.0, $121.1,120.0,118.7,117.7,113.0,111.6,107.9,54.4,43.4,29.9,19.3$.

Accurate mass (ESI, $\boldsymbol{m} / z)$ : calc'd for $\mathrm{C}_{19} \mathrm{H}_{18} \mathrm{~N}_{3} \mathrm{O}\left([\mathrm{M}+\mathrm{H}]^{+}\right)$: 304.1450, found $\mathrm{m} / \mathrm{z}$ 304.1456.

TLC (SiO $): R_{\mathrm{f}}=0.57(40 \%$ EtOAc in $n$-pentane). 


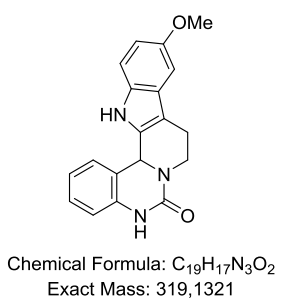

\section{1-Methoxy-8,9,14,14b-tetrahydroindolo[ $\left.2^{\prime}, 3^{\prime}: 3,4\right]$ pyrido[1,2-c]quinazolin-6(5H)-one}

Prepared following the general procedure (A) starting from aldehyde 1a (25 mg, $140 \mu \mathrm{mol})$ and amine $3 \mathbf{b}$ (35 mg, $184 \mu \mathrm{mol})$.

Physical state and yield: yellow solid (34 mg, $106 \mu \mathrm{mol}, 75 \%)$.

${ }^{1}$ H NMR (400 MHz, DMSO-d $)$ ): $\delta 10.20$ (s, 1H), 9.45 (s, 1H), 7.49-7.44 (m, 1H), 7.25 (dd, $J$ $=7.7,1.4 \mathrm{~Hz}, 1 \mathrm{H}), 7.21(\mathrm{~d}, J=8.8 \mathrm{~Hz}, 1 \mathrm{H}), 7.04(\mathrm{td}, J=7.5,1.2 \mathrm{~Hz}, 1 \mathrm{H}), 6.89(\mathrm{~d}, J=2.5 \mathrm{~Hz}$, 1H), $6.81(\mathrm{dd}, J=7.9,1.2 \mathrm{~Hz}, 1 \mathrm{H}), 6.68(\mathrm{dd}, J=8.7,2.5 \mathrm{~Hz}, 1 \mathrm{H}), 5.99(\mathrm{~s}, 1 \mathrm{H}), 4.57-4.37$ (m, $1 \mathrm{H}), 3.74(\mathrm{~s}, 3 \mathrm{H}), 3.13(\mathrm{td}, J=12.5,4.5 \mathrm{~Hz}, 1 \mathrm{H}), 3.01-2.89(\mathrm{~m}, 1 \mathrm{H}), 2.66-2.56(\mathrm{~m}, 1 \mathrm{H})$.

${ }^{13}$ C NMR (100 MHz, DMSO-d $): \delta$ 154.0, 153.2, 136.9, 133.3, 131.0, 128.6, 127.1, 126.9, $121.5,117.8,113.5,112.3,111.0,107.6,99.7,79.2,55.4,55.3,42.4,19.5$.

Accurate mass (ESI, $\boldsymbol{m} / \boldsymbol{z}$ ): calc'd for $\mathrm{C}_{19} \mathrm{H}_{18} \mathrm{~N}_{3} \mathrm{O}_{2}\left([\mathrm{M}+\mathrm{H}]^{+}\right)$: 320.1399 , found $\mathrm{m} / \mathrm{z} 320.1396$.

TLC $\left(\mathrm{SiO}_{2}\right): R_{\mathrm{f}}=0.28(50 \%$ EtOAc in $n$-pentane $)$.

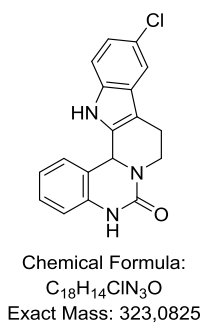

11-Chloro-8,9,14,14b-tetrahydroindolo[ $\left.2^{\prime}, 3^{\prime}: 3,4\right]$ pyrido[1,2-c]quinazolin-6(5H)-one (23)

Prepared following the general procedure (B), starting from aldehyde $\mathbf{1 a}(20 \mathrm{mg}, 112 \mu \mathrm{mol})$ and amine 3c (34 mg, $147 \mu \mathrm{mol}$, as the hydrochloride salt). 
Physical state and yield: white solid (32 mg, $100 \mu \mathrm{mol}, 89 \%)$.

${ }^{1}$ H NMR (400 MHz, CDCl3): $\delta 10.58(\mathrm{~s}, 1 \mathrm{H}), 9.49$ (s, 1H), 7.52-7.43 (m, 2H), 7.33 (d, $J=$ $8.6 \mathrm{~Hz}, 1 \mathrm{H}), 7.30-7.21(\mathrm{~m}, 1 \mathrm{H}), 7.10-7.01(\mathrm{~m}, 2 \mathrm{H}), 6.83-6.79(\mathrm{~m}, 1 \mathrm{H}), 6.03(\mathrm{~s}, 1 \mathrm{H}), 4.48$ $(\mathrm{dd}, J=13.0,5.6 \mathrm{~Hz}, 1 \mathrm{H}), 3.14(\mathrm{td}, J=12.4,4.5 \mathrm{~Hz}, 1 \mathrm{H}), 3.02-2.87(\mathrm{~m}, 1 \mathrm{H}), 2.70-2.54(\mathrm{~m}$, $1 \mathrm{H})$.

${ }^{13}$ C NMR (100 MHz, CDCl3): $\delta$ 153.9, 151.0, 136.9, 134.7, 134.4, 128.8, 127.9, 127.0, $123.4,121.5,121.0,117.4,117.0,113.5,113.1,107.8,55.2,42.3,19.2$.

Accurate mass (ESI, $\boldsymbol{m} / \boldsymbol{z})$ : calc'd for $\mathrm{C}_{20} \mathrm{H}_{18} \mathrm{ClN} \mathrm{N}_{4} \mathrm{O}\left([\mathrm{M}+\mathrm{MeCN}+\mathrm{H}]^{+}\right): 365.1169$, found $\mathrm{m} / \mathrm{z}$ 365.1169.

TLC (SiO $): R_{\mathrm{f}}=0.20(50 \%$ EtOAc in $n$-pentane).

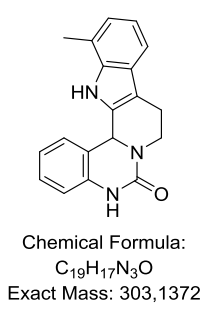

13-Methyl-8,9,14,14b-tetrahydroindolo[2',3':3,4]pyrido[1,2-c]quinazolin-6(5H)-one (24) Prepared following the general procedure (A) but with $40 \mathrm{~min}$ of heating, starting from aldehyde 1a (25 mg, $140 \mu \mathrm{mol})$ and amine 3d (32 mg, $184 \mu \mathrm{mol})$.

Physical state and yield: yellow solid (32 mg, $106 \mu \mathrm{mol}, 75 \%)$.

${ }^{1}$ H NMR (400 MHz, CDCl3): $\delta 10.27$ (s, 1H), 9.52 (s, 1H), 7.55 (d, J = 7.6 Hz, 1H), 7.30 $7.18(\mathrm{~m}, 2 \mathrm{H}), 7.02(\mathrm{t}, J=7.5 \mathrm{~Hz}, 1 \mathrm{H}), 6.94-6.83(\mathrm{~m}, 3 \mathrm{H}), 5.90(\mathrm{~s}, 1 \mathrm{H}), 4.47(\mathrm{dd}, J=12.8,5.2$ $\mathrm{Hz}, 1 \mathrm{H}), 3.12-3.02(\mathrm{~m}, 1 \mathrm{H}), 2.91-2.81(\mathrm{~m}, 1 \mathrm{H}), 2.72-2.64(\mathrm{~m}, 1 \mathrm{H}), 2.45$ (s, 3H).

${ }^{13}$ C NMR (100 MHz, CDCl3): $\delta$ 154.7, 137.4, 135.6, 131.7, 126.5, 126.5, 122.1, 121.3, 120.7, 119.3, 119.1, 115.4, 113.4, 109.3, 54.5, 41.7, 19.9, 17.1 . 
Accurate mass (ESI, $\boldsymbol{m} / z)$ : calc' $d$ for $\mathrm{C}_{19} \mathrm{H}_{17} \mathrm{~N}_{3} \mathrm{O}\left([\mathrm{M}+\mathrm{H}]^{+}\right)$: 304.1450, found $\mathrm{m} / z$ 304.1449.

TLC (SiO $): R_{\mathrm{f}}=0.24(40 \%$ EtOAc in $n$-pentane).

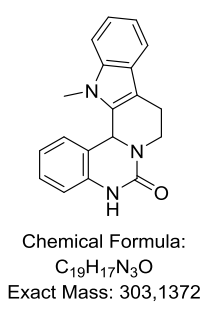

14-Methyl-8,9,14,14b-tetrahydroindolo[2',3':3,4]pyrido[1,2-c]quinazolin-6(5H)-one (25) Prepared following the general procedure $(\mathbf{B})$ but with $30 \mathrm{~min}$ of heating, starting from aldehyde 1a (20 mg, $112 \mu \mathrm{mol})$ and amine 3e (36 mg, $146 \mu \mathrm{mol})$.

Physical state and yield: yellow solid (29 mg, $95 \mu \mathrm{mol}, 86 \%$ ). A yield of 35\% was obtained when using the hydrochloride salt of the amine directly following general procedure (A).

${ }^{1}$ H NMR (400 MHz, CDCl $): \delta 9.63(\mathrm{~s}, 1 \mathrm{H}), 7.56-7.51(\mathrm{~m}, 1 \mathrm{H}), 7.51-7.46(\mathrm{~m}, 1 \mathrm{H}), 7.27-$ $7.17(\mathrm{~m}, 2 \mathrm{H}), 7.12-7.05(\mathrm{~m}, 1 \mathrm{H}), 7.02-6.97(\mathrm{~m}, 1 \mathrm{H}), 6.96-6.88(\mathrm{~m}, 2 \mathrm{H}), 5.79(\mathrm{~s}, 1 \mathrm{H}), 4.46-$ $4.26(\mathrm{~m}, 1 \mathrm{H}), 3.60(\mathrm{~s}, 3 \mathrm{H}), 3.02-2.90(\mathrm{~m}, 1 \mathrm{H}), 2.89-2.76(\mathrm{~m}, 1 \mathrm{H}), 2.74-2.59(\mathrm{~m}, 1 \mathrm{H})$.

${ }^{13}$ C NMR (100 MHz, CDCl 3$): \delta 156.1,138.8,137.6,131.5,128.3,126.1,124.3,122.0$, 121.43, 121.41, 119.1, 118.1, 113.8, 109.8, 109.5, 51.7, 40.4, 31.0, 20.6.

Accurate mass (ESI, $\boldsymbol{m} / z)$ : calc' $d$ for $\mathrm{C}_{19} \mathrm{H}_{18} \mathrm{~N}_{3} \mathrm{O}\left([\mathrm{M}+\mathrm{H}]^{+}\right)$: 304.1450, found $\mathrm{m} / \mathrm{z}$ 304.1438.

TLC (SiO2): $R_{\mathrm{f}}=0.20(50 \%$ EtOAc in $n$-pentane).

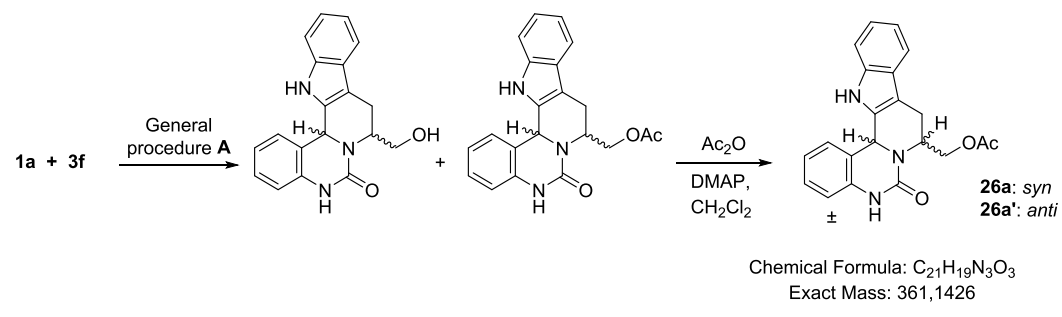




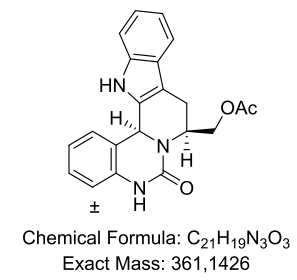

(syn)-6-Oxo-5,6,8,9,14,14b-hexahydroindolo[2',3' :3,4]pyrido[1,2-c]quinazolin-8yl)methyl acetate (26a)

Prepared following the general procedure (C), starting from aldehyde 1a (40 mg, $224 \mu \mathrm{mol}$ ) and amine $\mathbf{3 f}$ (55 mg, $295 \mu \mathrm{mol})$.

Physical state and yield: yellow solid (4 mg, $11 \mu \mathrm{mol}, 5 \%)$.

${ }^{1}$ H NMR (400 MHz, CDCl3): $\delta 7.69(\mathrm{~s}, 1 \mathrm{H}), 7.50-7.44(\mathrm{~m}, 2 \mathrm{H}), 7.38-7.34(\mathrm{~m}, 1 \mathrm{H}), 7.34-$ $7.27(\mathrm{~m}, 1 \mathrm{H}), 7.24-7.19(\mathrm{~m}, 1 \mathrm{H}), 7.16-7.03(\mathrm{~m}, 3 \mathrm{H}), 6.81-6.70(\mathrm{~m}, 1 \mathrm{H})$, 5.96-5.69 (m, 1H), $5.00(\mathrm{dd}, J=11.2,5.5 \mathrm{~Hz}, 1 \mathrm{H}), 4.86(\mathrm{dd}, J=11.3,7.1 \mathrm{~Hz}, 1 \mathrm{H}), 3.95-3.70(\mathrm{~m}, 1 \mathrm{H}), 3.50-3.26$ (m, 1H), 3.07-2.72 (m, 1H), $2.09(\mathrm{~s}, 3 \mathrm{H})$.

${ }^{13}$ C NMR (100 MHz, CDCl 3$): \delta 170.9,155.7,136.0,135.9,131.9,129.6,127.4,125.4$, $122.5,122.4,120.0,118.4,117.6,114.3,111.1,109.9,67.0,59.4,59.1,22.9,21.0$.

Accurate mass (ESI, $\boldsymbol{m} / z)$ : calc' $d$ for $\mathrm{C}_{21} \mathrm{H}_{20} \mathrm{~N}_{3} \mathrm{O}_{3}\left([\mathrm{M}+\mathrm{H}]^{+}\right)$: 362.1505 , found $m / z$ 362.1499.

TLC (SiO2): $R_{\mathrm{f}}=0.30(50 \%$ EtOAc in $n$-pentane $)$.

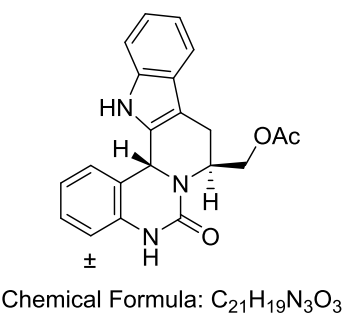

$$
\text { Exact Mass: } 361,1426
$$

(anti)-6-Oxo-5,6,8,9,14,14b-hexahydroindolo[2',3':3,4]pyrido[1,2-c]quinazolin-8yl)methyl acetate (26a').

Physical state and yield: white solid (45 mg, $125 \mu \mathrm{mol}, 56 \%)$. 
${ }^{1}$ H NMR (400 MHz, CDCl $)$ ) $\delta 8.52$ (s, 1H), 7.95 (s, 1H), 7.50-7.43 (m, 1H), 7.39-7.32 (m, 1H), 7.33-7.28 (m, 1H), 7.25-7.21 (m, 1H), 7.19-7.13 (m, 1H), 7.13-7.03 (m, 2H), 6.79 (dd, $J=7.9,1.1 \mathrm{~Hz}, 1 \mathrm{H}), 5.99-5.58(\mathrm{~m}, 1 \mathrm{H}), 5.44-5.14(\mathrm{~m}, 1 \mathrm{H}), 4.42(\mathrm{dd}, J=11.5,8.9 \mathrm{~Hz}, 1 \mathrm{H})$, $4.21(\mathrm{dd}, J=11.5,6.3 \mathrm{~Hz}, 1 \mathrm{H}), 3.25(\mathrm{ddd}, J=16.2,6.7,2.4 \mathrm{~Hz}, 1 \mathrm{H}), 2.84-2.56(\mathrm{~m}, 1 \mathrm{H})$, $2.08-1.93(\mathrm{~m}, 3 \mathrm{H})$.

${ }^{13}$ C NMR (100 MHz, CDCl 3$): \delta 171.0,155.2,136.3,136.0,129.8,129.3,127.5,125.3$, $122.4,119.9,118.3,117.7,114.5,111.2,107.6,62.4,50.9,48.4,21.2,20.9$.

Accurate mass (ESI, $\boldsymbol{m} / \boldsymbol{z}$ ): calc' $\mathrm{d}$ for $\mathrm{C}_{21} \mathrm{H}_{20} \mathrm{~N}_{3} \mathrm{O}_{3}\left([\mathrm{M}+\mathrm{H}]^{+}\right)$: 362.1505 , found $\mathrm{m} / z$ 362.1507.

TLC $\left(\mathrm{SiO}_{2}\right): R_{\mathrm{f}}=0.12(50 \%$ EtOAc in $n$-pentane)
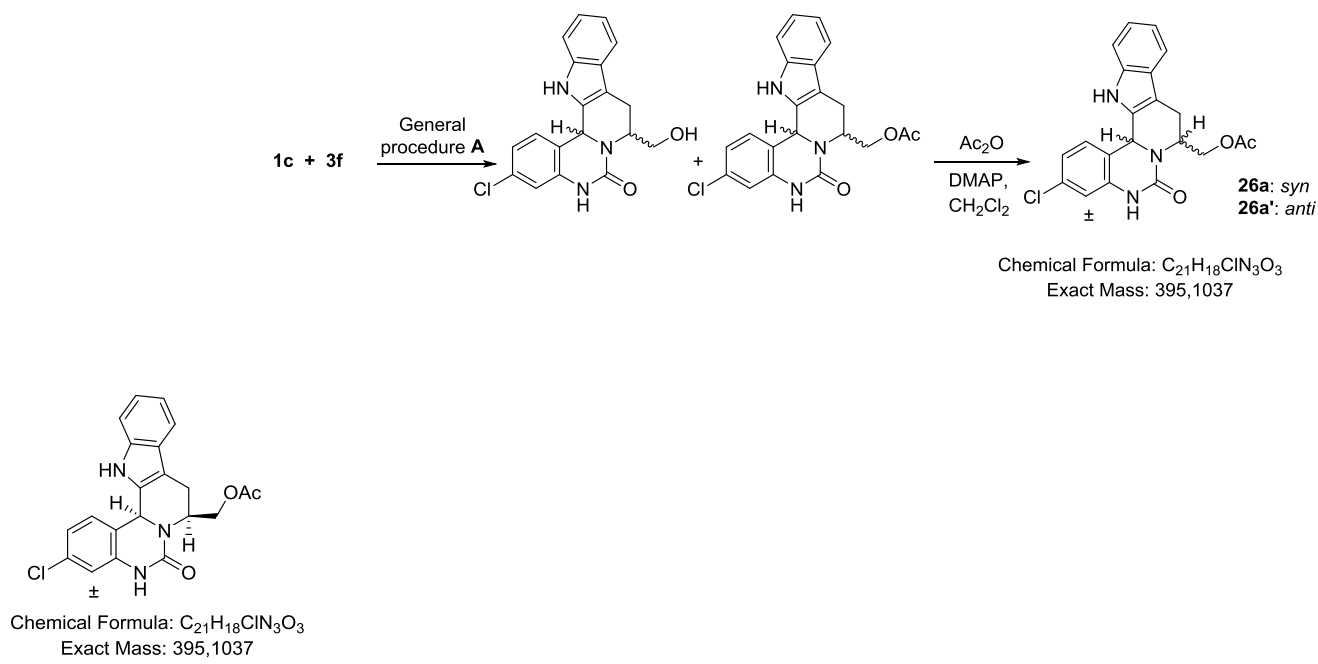

(syn)-3-Chloro-6-oxo-5,6,8,9,14,14b-hexahydroindolo[2',3':3,4]pyrido[1,2-c]quinazolin8-yl)methyl acetate (26b).

Prepared following general procedure $\mathbf{C}$, starting from aldehyde $1 \mathbf{c}(40 \mathrm{mg}, 187 \mu \mathrm{mol})$ and amine 3f (46 mg, $242 \mu \mathrm{mol})$.

Physical state and yield: yellow solid (4 mg, $10 \mu \mathrm{mol}, 6 \%)$.

${ }^{1}$ H NMR (400 MHz, CDCl3): $\delta$ 7.83-7.68 (m, 1H), 7.50-7.43 (m, 2H), 7.31-7.27 (m, 1H), 7.25-7.21 (m, 1H), 7.17-7.06 (m, 3H), $6.74(\mathrm{~d}, J=2.0 \mathrm{~Hz}, 1 \mathrm{H}), 5.85-5.73(\mathrm{~m}, 1 \mathrm{H}), 4.98(\mathrm{dd}$, 
$J=11.3,5.6 \mathrm{~Hz}, 1 \mathrm{H}), 4.82(\mathrm{dd}, J=11.3,7.0 \mathrm{~Hz}, 1 \mathrm{H}), 3.81(\mathrm{dt}, J=11.2,5.6 \mathrm{~Hz}, 1 \mathrm{H}), 3.34$ (ddd, $J=15.5,11.2,2.5 \mathrm{~Hz}, 1 \mathrm{H}), 2.94$ (ddd, $J=15.5,4.4,2.0 \mathrm{~Hz}, 1 \mathrm{H}), 2.08(\mathrm{~s}, 3 \mathrm{H})$.

${ }^{13}$ C NMR (100 MHz, CDCl3): $\delta$ 171.0, 155.4, 137.4, 136.1, 135.5, 131.4, 127.4, 126.7, 122.7, 122.6, 120.3, 118.6, 116.3, 114.5, 111.3, 110.3, 66.9, 58.8, 29.8, 23.0.

Accurate mass (ESI, $\boldsymbol{m} / z)$ : calc'd for $\mathrm{C}_{21} \mathrm{H}_{19} \mathrm{ClN}_{3} \mathrm{O}_{3}\left([\mathrm{M}+\mathrm{H}]^{+}\right)$: 396.1115, found $\mathrm{m} / \mathrm{z}$ 396.1102.

TLC (SiO $): R_{\mathrm{f}}=0.48(40 \%$ EtOAc in $n$-pentane)

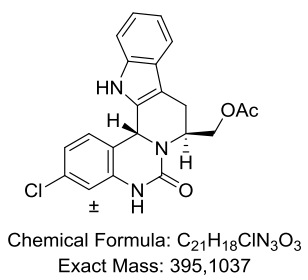

(anti)-3-Chloro-6-oxo-5,6,8,9,14,14b-hexahydroindolo[2',3':3,4]pyrido[1,2-c]quinazolin8-yl)methyl acetate (26b').

Prepared following general procedure C, starting from aldehyde 1c (40 mg, $187 \mu \mathrm{mol})$ and amine 3f (46 mg, $242 \mu \mathrm{mol})$.

Physical state and yield: yellow solid (32 mg, $81 \mu \mathrm{mol}, 43 \%)$.

${ }^{1}$ H NMR (400 MHz, CDCl $)$ ) $\delta 8.70(\mathrm{~s}, 1 \mathrm{H}), 7.95$ (s, 1H), 7.51-7.45 (m, 1H), 7.33-7.24 (m, 2H), 7.20-7.15 (m, 1H), 7.14-7.08 (m, 1H), $7.03(\mathrm{dd}, J=8.1,2.0 \mathrm{~Hz}, 1 \mathrm{H}), 6.81(\mathrm{~d}, J=2.0$ $\mathrm{Hz}, 1 \mathrm{H}), 5.82(\mathrm{~d}, J=1.9 \mathrm{~Hz}, 1 \mathrm{H}), 5.36-5.22(\mathrm{~m}, 1 \mathrm{H}), 4.42(\mathrm{dd}, J=11.5,8.9 \mathrm{~Hz}, 1 \mathrm{H}), 4.18$ (dd, $J=11.6,6.2 \mathrm{~Hz}, 1 \mathrm{H}), 3.23$ (ddd, $J=16.3,6.7,2.3 \mathrm{~Hz}, 1 \mathrm{H}), 2.79-2.63(\mathrm{~m}, 1 \mathrm{H}), 2.03$ (s, $3 \mathrm{H})$.

${ }^{13}$ C NMR (100 MHz, CDCl3): $\delta$ 171.0, 155.0, 137.6, 136.1, 135.0, 129.2, 127.4, 126.4, $122.6,122.4,120.0,118.4,116.4,114.5,111.2,107.8,62.4,50.6,48.6,21.2,20.9$. 
Accurate mass (ESI, m/z): calc'd for $\mathrm{C}_{21} \mathrm{H}_{17} \mathrm{ClN}_{3} \mathrm{O}_{3}\left([\mathrm{M}-\mathrm{H}]^{-}\right)$: 394.0958 , found $\mathrm{m} / \mathrm{z}$ 394.0946.

TLC (SiO2): $R_{\mathrm{f}}=0.26(40 \%$ EtOAc in $n$-pentane).

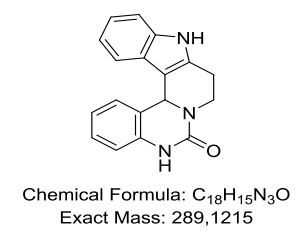

8,9,10,14c-Tetrahydroindolo[3',2':3,4]pyrido[1,2-c]quinazolin-6(5H)-one (27a).

Prepared following the general procedure (A) starting from aldehyde $1 \mathbf{a}(20 \mathrm{mg}, 112 \mu \mathrm{mol})$ and amine 4 (23 mg, $144 \mu \mathrm{mol})$.

Physical state and yield: yellow solid (14 mg, $48 \mu \mathrm{mol}, 43 \%)$.

${ }^{1}$ H NMR (400 MHz, DMSO-d6): $\delta 11.15$ (s, 1H), 9.50 (s, 1H), 7.48-7.44 (m, 1H), 7.36-7.32 (m, 1H), 7.28-7.22 (m, 1H), 7.18 (d, J = 8.0 Hz, 1H), 7.08-6.98 (m, 2H), 6.92-6.86 (m, 2H), $5.86(\mathrm{~s}, 1 \mathrm{H}), 4.46(\mathrm{dd}, J=12.4,5.3 \mathrm{~Hz}, 1 \mathrm{H}), 3.25-3.02(\mathrm{~m}, 2 \mathrm{H}), 2.82-2.67(\mathrm{~m}, 1 \mathrm{H})$.

${ }^{13}$ C NMR (100 MHz, DMSO-d6): $\delta$ 156.0, 138.1, 136.0, 134.5, 128.7, 127.0, 125.5, 121.7, $121.3,120.7,119.0,118.7,114.0,111.5,107.7,55.2,41.6,22.3$.

Accurate mass (ESI, $\boldsymbol{m} / z)$ : calc'd for $\mathrm{C}_{20} \mathrm{H}_{19} \mathrm{~N}_{4} \mathrm{O}\left([\mathrm{M}+\mathrm{MeCN}+\mathrm{H}]^{+}\right): 331.1559$, found $\mathrm{m} / z$ 331.1555 .

TLC (SiO2): $R_{\mathrm{f}}=0.10(50 \%$ EtOAc in $n$-pentane)

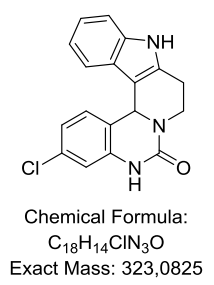


3-Chloro-8,9,10,14c-tetrahydroindolo[3',2':3,4]pyrido[1,2-c]quinazolin-6(5H)-one (27b). Prepared following the general procedure (A) starting from aldehyde 1c (20 mg, $94 \mu \mathrm{mol})$ and amine 4 (20 mg, $125 \mu \mathrm{mol})$.

Physical state and yield: yellow solid (18 mg, $56 \mu \mathrm{mol}, 59 \%)$.

${ }^{1}$ H NMR (400 MHz, DMSO-d6): $\delta 11.17$ (s, 1H), 9.65 (s, 1H), 7.48-7.39 (m, 1H), 7.36-7.30 (m, 1H), 7.18-7.13 (m, 1H), $7.07(\mathrm{dd}, J=8.1,2.1 \mathrm{~Hz}, 1 \mathrm{H}), 7.05-6.99(\mathrm{~m}, 1 \mathrm{H}), 6.94-6.85$ (m, $3 \mathrm{H}), 5.86(\mathrm{~s}, 1 \mathrm{H}), 4.60-4.21(\mathrm{~m}, 1 \mathrm{H}), 3.21-3.12(\mathrm{~m}, 1 \mathrm{H}), 3.11-3.01(\mathrm{~m}, 1 \mathrm{H}), 2.80-2.71(\mathrm{~m}$, $1 \mathrm{H})$.

${ }^{13}$ C NMR (100 MHz, DMSO-d $): \delta$ 155.1, 139.3, 135.6, 134.2, 132.5, 128.3, 125.0, 120.6, $120.4,120.4,118.8,118.2,113.1,111.2,106.7,54.2,41.1,21.9$.

Accurate mass (ESI, $\boldsymbol{m} / \boldsymbol{z})$ : calc'd for $\mathrm{C}_{20} \mathrm{H}_{18} \mathrm{ClN} \mathrm{N}_{4} \mathrm{O}\left([\mathrm{M}+\mathrm{MeCN}+\mathrm{H}]^{+}\right): 365.1169$, found $\mathrm{m} / \mathrm{z}$ 365.1149.

TLC (SiO2): $R_{\mathrm{f}}=0.15(50 \%$ EtOAc in $n$-pentane).

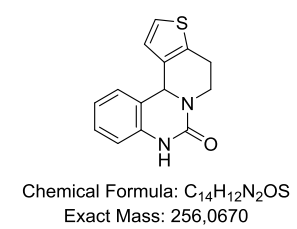

4,5,8,12b-Tetrahydro-7H-thieno[ $\left.2^{\prime}, 3 ': 3,4\right]$ pyrido[1,2-c]quinazolin-7-one (CAS 69103-365) $(\mathbf{2 8 a})^{[2]}$

Prepared following the general procedure (A) starting from aldehyde 1a (40 mg, $224 \mu \mathrm{mol}$ ) and amine 5 (40 mg, $250 \mu \mathrm{mol})$.

Physical state and yield: yellow solid (40 mg, $156 \mu \mathrm{mol}, 73 \%)$.

${ }^{1}$ H NMR (400 MHz, DMSO-d6): $\delta 9.46$ (s, 1H), 7.44-7.37 (m, 1H), 7.24 (d, $\left.J=5.2 \mathrm{~Hz}, 1 \mathrm{H}\right)$, $7.22-7.17(\mathrm{~m}, 1 \mathrm{H}), 7.02-6.97(\mathrm{~m}, 1 \mathrm{H}), 6.82-6.77(\mathrm{~m}, 1 \mathrm{H}), 6.62(\mathrm{~d}, J=5.2 \mathrm{~Hz}, 1 \mathrm{H}), 6.00$ 
$5.57(\mathrm{~m}, 1 \mathrm{H}), 4.43(\mathrm{dd}, J=13.0,5.3 \mathrm{~Hz}, 1 \mathrm{H}), 3.21-3.11(\mathrm{~m}, 1 \mathrm{H}), 3.11-2.99(\mathrm{~m}, 1 \mathrm{H}), 2.83-$ $2.70(\mathrm{~m}, 1 \mathrm{H})$.

${ }^{13}$ C NMR (100 MHz, DMSO-d6): $\delta$ 153.7, 136.7, 135.8, 134.5, 128.4, 127.0, 124.7, 122.7, $121.3,119.2,113.6,56.9,42.0,22.7$.

Accurate mass (ESI, $\boldsymbol{m} / z)$ : calc'd for $\mathrm{C}_{14} \mathrm{H}_{13} \mathrm{~N}_{2} \mathrm{OS}\left([\mathrm{M}+\mathrm{H}]^{+}\right)$: 257.0749 , found $m / z, 257.0751$.

TLC (SiO2): $R_{\mathrm{f}}=0.15(50 \%$ EtOAc in $n$-pentane).

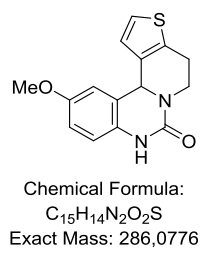

11-Methoxy-4,5,8,12b-tetrahydro-7H-thieno[ $\left[3^{\prime}, 2^{\prime}: 3,4\right]$ pyrido[1,2-c]quinazolin-7-one (28b)

Prepared following the general procedure (A) starting from aldehyde $\mathbf{1 b}(20 \mathrm{mg}, 96 \mu \mathrm{mol})$ and amine 5 (16 mg, $126 \mu \mathrm{mol})$.

Physical state and yield: yellow solid (18 mg, $59 \mu \mathrm{mol}, 62 \%)$.

${ }^{1}$ H NMR (400 MHz, DMSO-d6): $\delta 9.32(\mathrm{~s}, 1 \mathrm{H}), 7.28(\mathrm{~d}, J=5.2 \mathrm{~Hz}, 1 \mathrm{H}), 7.05$ (d, $J=2.7 \mathrm{~Hz}$, $1 \mathrm{H}), 6.86(\mathrm{dd}, J=8.7,2.7 \mathrm{~Hz}, 1 \mathrm{H}), 6.76(\mathrm{~d}, J=8.6 \mathrm{~Hz}, 1 \mathrm{H}), 6.72(\mathrm{~d}, J=5.2 \mathrm{~Hz}, 1 \mathrm{H}), 4.57-$ $4.35(\mathrm{~m}, 1 \mathrm{H}), 3.79(\mathrm{~s}, 3 \mathrm{H}), 3.29-3.01(\mathrm{~m}, 2 \mathrm{H}), 2.87-2.71(\mathrm{~m}, 1 \mathrm{H})$.

${ }^{13}$ C NMR (100 MHz, DMSO-d $): \delta$ 154.0, 153.8, 135.6, 134.6, 130.2, 124.9, 122.6, 120.2, 114.6, 114.1, 112.3, 57.1, 55.4, 42.0, 22.7.

Accurate mass (ESI, $\boldsymbol{m} / \boldsymbol{z})$ : calc'd for $\mathrm{C}_{17} \mathrm{H}_{18} \mathrm{~N}_{3} \mathrm{O}_{2} \mathrm{~S}\left([\mathrm{M}+\mathrm{MeCN}+\mathrm{H}]^{+}\right): 328.1120$, found $\mathrm{m} / z$ 328.1124 .

TLC $\left(\mathbf{S i O}_{2}\right): R_{\mathrm{f}}=0.20(50 \%$ EtOAc in $n$-pentane $)$. 


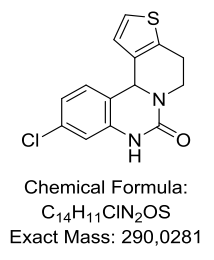

10-Chloro-4,5,8,12b-tetrahydro-7H-thieno[3' $2^{\prime}$ ':3,4]pyrido[1,2-c]quinazolin-7-one (28c)

Prepared following the general procedure (A) starting from aldehyde $1 \mathbf{c}(20 \mathrm{mg}, 94 \mu \mathrm{mol})$ and amine 5 (15 mg, $118 \mu \mathrm{mol})$.

Physical state and yield: white solid (22 mg, $76 \mu \mathrm{mol}, 81 \%)$.

${ }^{1}$ H NMR (400 MHz, DMSO-d6): $\delta 9.67$ (s, 1H), 7.48 (d, $\left.J=8.2 \mathrm{~Hz}, 1 \mathrm{H}\right), 7.30$ (d, $J=5.3 \mathrm{~Hz}$, 1H), $7.08(\mathrm{dd}, J=8.2,2.1 \mathrm{~Hz}, 1 \mathrm{H}), 6.87(\mathrm{~d}, J=2.1 \mathrm{~Hz}, 1 \mathrm{H}), 6.66(\mathrm{~d}, J=5.3 \mathrm{~Hz}, 1 \mathrm{H}), 6.14-$ $5.65(\mathrm{~m}, 1 \mathrm{H}), 3.25-3.12(\mathrm{~m}, 1 \mathrm{H}), 3.11-2.98(\mathrm{~m}, 1 \mathrm{H}), 2.87-2.72(\mathrm{~m}, 1 \mathrm{H})$.

${ }^{13}$ C NMR (100 MHz, DMSO-d6): $\delta$ 153.3, 138.3, 135.4, 134.7, 132.7, 128.8, 124.6, 123.0, 121.0, 118.2, 113.1, 56.4, 42.0, 22.7.

Accurate mass (ESI, $\boldsymbol{m} / \boldsymbol{z})$ : calc'd for $\mathrm{C}_{16} \mathrm{H}_{15} \mathrm{ClN}_{3} \mathrm{OS}\left([\mathrm{M}+\mathrm{MeCN}+\mathrm{H}]^{+}\right): 332.0624$, found $\mathrm{m} / z$ 336.0636.

TLC (SiO2): $R_{\mathrm{f}}=0.30(50 \%$ EtOAc in $n$-pentane)

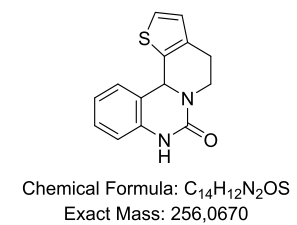

4,5,8,12b-Tetrahydro-7H-thieno[2',3':3,4]pyrido[1,2-c]quinazolin-7-one (CAS 96120-717) $(29 a)^{[2]}$

Prepared following the general procedure (A) but with 40 min of heating, starting from aldehyde 1a (20 mg, $112 \mu \mathrm{mol})$ and amine 6 (18 mg, $141 \mu \mathrm{mol})$.

Physical state and yield: white solid (23 mg, $109 \mu \mathrm{mol}, 80 \%)$. 
${ }^{1}$ H NMR (400 MHz, DMSO-d $)$ ): $\delta 9.49$ (s, 1H), 7.37-7.33 (m, 1H), 7.31 (d, $\left.J=5.1 \mathrm{~Hz}, 1 \mathrm{H}\right)$, 7.25-7.16 (m, 1H), 7.05-6.97 (m, 1H), $6.86(\mathrm{~d}, J=5.1 \mathrm{~Hz}, 1 \mathrm{H}), 6.83-6.76(\mathrm{~m}, 1 \mathrm{H}), 5.97(\mathrm{~s}$, $1 \mathrm{H}), 4.40(\mathrm{dd}, J=13.3,6.2 \mathrm{~Hz}, 1 \mathrm{H}), 3.24-3.07(\mathrm{~m}, 1 \mathrm{H}), 3.03-2.79(\mathrm{~m}, 1 \mathrm{H}), 2.71-2.54(\mathrm{~m}$, $1 \mathrm{H})$.

${ }^{13}$ C NMR (100 MHz, DMSO-d6): $\delta$ 153.2, 136.7, 136.4, 134.5, 128.9, 128.0, 126.1, 123.8, $121.4,119.4,113.7,56.6,41.9,23.4$.

Accurate mass (ESI, $\boldsymbol{m} / z)$ : calc'd for $\mathrm{C}_{14} \mathrm{H}_{13} \mathrm{~N}_{2} \mathrm{OS}\left([\mathrm{M}+\mathrm{H}]^{+}\right)$: 257.0749 , found $m / z$ 257.0753.

TLC (SiO2): $R_{\mathrm{f}}=0.33(50 \%$ EtOAc in $n$-pentane)

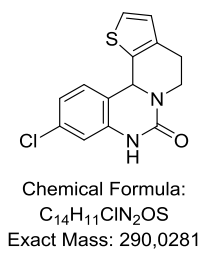

10-Chloro-4,5,8,12b-tetrahydro-7H-thieno[2',3' :3,4]pyrido[1,2-c]quinazolin-7-one (29b)

Prepared following the general procedure (A) but with 40 min of heating, starting from aldehyde 1c (20 mg, $94 \mu \mathrm{mol}$ ) and amine 6 (hydrochloride salt, $20 \mathrm{mg}, 122 \mu \mathrm{mol}$ ).

Physical state and yield: white solid (17 mg, $59 \mu \mathrm{mol}, 63 \%)$.

${ }^{1}$ H NMR (400 MHz, DMSO-d6): 9.71 (s, 1H), 7.44 (d, $\left.J=8.1 \mathrm{~Hz}, 1 \mathrm{H}\right), 7.37$ (d, $J=5.1 \mathrm{~Hz}$, 1H), $7.11(\mathrm{dd}, J=8.1,2.1 \mathrm{~Hz}, 1 \mathrm{H}), 6.91(\mathrm{~d}, J=5.1 \mathrm{~Hz}, 1 \mathrm{H}), 6.87(\mathrm{~d}, J=2.1 \mathrm{~Hz}, 1 \mathrm{H}), 6.04(\mathrm{~s}$, 1H), $4.43(\mathrm{dd}, J=13.3,6.1 \mathrm{~Hz}, 1 \mathrm{H}), 3.30-3.09(\mathrm{~m}, 1 \mathrm{H}), 3.05-2.86(\mathrm{~m}, 1 \mathrm{H}), 2.70-2.60(\mathrm{~m}$, $1 \mathrm{H})$.

${ }^{13}$ C NMR (100 MHz, DMSO-d6): $\delta$ 152.8, 138.0, 136.1, 134.6, 133.1, 128.1, 127.9, 124.0, $121.1,118.4,113.2,56.1,42.0,23.4$.

Accurate mass (ESI, $\boldsymbol{m} / \boldsymbol{z})$ : calc'd for $\mathrm{C}_{16} \mathrm{H}_{15} \mathrm{ClN}_{3} \mathrm{OS}\left([\mathrm{M}+\mathrm{MeCN}+\mathrm{H}]^{+}\right): 332.0624$, found $\mathrm{m} / \mathrm{z}$ 332.0626. 
TLC (SiO $): R_{\mathrm{f}}=0.30(50 \%$ EtOAc in $n$-pentane)

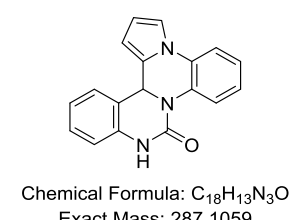

Exal Formula: $\mathrm{C}_{18} \mathrm{H}_{13} \mathrm{~N}_{3} \mathrm{C}$

\section{7,11b-Dihydro-6H-pyrrolo[2',1':3,4]quinoxalino[1,2-c]quinazolin-6-one (30a)}

Prepared following the general procedure (A) but with $120 \mathrm{~min}$ of heating, starting from aldehyde 1a (40 mg, $224 \mu \mathrm{mol})$ and amine 7 (46 mg, $291 \mu \mathrm{mol})$.

Physical state and yield: white solid (51 mg, $178 \mu \mathrm{mol}, 80 \%)$.

${ }^{1}$ H NMR (400 MHz, CDCl3): $\delta$ 7.74-7.65 (m, 1H), 7.48-7.43 (m, 1H), 7.36-7.29 (m, 2H), 7.29-7.24 (m, 2H), 7.22-7.19 (m, 1H), $7.07(\mathrm{td}, J=7.5,1.1 \mathrm{~Hz}, 1 \mathrm{H}), 6.77(\mathrm{dd}, J=8.0,1.1$ $\mathrm{Hz}, 1 \mathrm{H}), 6.38-6.07(\mathrm{~m}, 1 \mathrm{H}), 5.83(\mathrm{~s}, 1 \mathrm{H}), 5.76(\mathrm{dt}, J=3.5,1.4 \mathrm{~Hz}, 1 \mathrm{H})$.

${ }^{13}$ C NMR (100 MHz, CDCl3): $\delta$ 151.1, 135.4, 131.7, 130.1, 129.3, 128.9, 127.5, 127.1, $126.9,124.1,122.3,116.2,115.9,115.4,114.5,110.2,105.1,55.5$.

Accurate mass (ESI, $\boldsymbol{m} / \boldsymbol{z})$ : calc'd for $\mathrm{C}_{18} \mathrm{H}_{14} \mathrm{~N}_{3} \mathrm{O}\left([\mathrm{M}+\mathrm{H}]^{+}\right)$: 288.1137 , found $m / z 288.1142$.

TLC (SiO2): $R_{\mathrm{f}}=0.33(40 \%$ EtOAc in $n$-pentane).

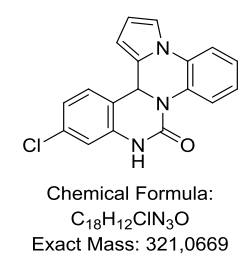

9-Chloro-7,11b-dihydro-6H-pyrrolo[2',1':3,4]quinoxalino[1,2-c]quinazolin-6-one (30b) Prepared following the general procedure (A) but with $120 \mathrm{~min}$ of heating, starting from aldehyde 1b (40 mg, $187 \mu \mathrm{mol})$ and amine 7 (39 mg, $246 \mu \mathrm{mol})$.

Physical state and yield: yellow solid (43 mg, $134 \mu \mathrm{mol}, 71 \%)$. 
${ }^{1}$ H NMR (400 MHz, DMSO-d6): $\delta 10.07$ (s, 1H), 7.78 (dd, $\left.J=8.1,1.3 \mathrm{~Hz}, 1 \mathrm{H}\right), 7.62$ - 7.59 (m, 1H), 7.59-7.55 (m, 1H), 7.46-7.39 (m, 2H), 7.32-7.23 (m, 1H), $7.15(\mathrm{dd}, J=8.1,2.1 \mathrm{~Hz}$ 1H), $6.99(\mathrm{~d}, J=2.1 \mathrm{~Hz}, 1 \mathrm{H}), 6.39-6.15(\mathrm{~m}, 1 \mathrm{H}), 5.99(\mathrm{~s}, 1 \mathrm{H}), 5.73-5.49(\mathrm{~m}, 1 \mathrm{H})$.

${ }^{13}$ C NMR (100 MHz, DMSO-d $)$ : $\delta$ 150.2, 137.7, 133.3, 131.3, 129.6, 129.1, 128.6, 127.8, 127.0, 123.8, 121.4, 116.9, 116.1, 114.4, 113.7, 110.1, 104.6, 53.9.

Accurate mass (ESI, m/z): calc'd for $\mathrm{C}_{18} \mathrm{H}_{13} \mathrm{ClN}_{3} \mathrm{O}\left([\mathrm{M}+\mathrm{H}]^{+}\right)$: 322.0747, found $\mathrm{m} / \mathrm{z}$ 322.0763.

TLC (SiO $): R_{\mathrm{f}}=0.21(25 \%$ EtOAc in $n$-pentane).

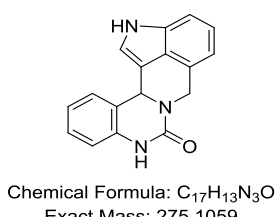

2,6,9,13b-Tetrahydro-8H-pyrrolo[4',3',2':4,5]isoquinolino[2,3-c]quinazolin-8-one (31a) Prepared following the general procedure (A) but with $40 \mathrm{~min}$ of heating, starting from aldehyde 1a (40 mg, $224 \mu \mathrm{mol})$ and amine 7 (42 mg, $287 \mu \mathrm{mol})$.

Physical state and yield: white solid (45 mg, $164 \mu \mathrm{mol}, 73 \%)$.

${ }^{1}$ H NMR (400 MHz, DMSO-d6): $\delta 10.76$ (s, 1H), 9.33 (s, 1H), 7.45-7.35 (m, 1H), 7.25-7.14 (m, 2H), 7.09-6.98 (m, 2H), 6.87-6.77 (m, 2H), 6.68-6.61 (m, 1H), $6.17(\mathrm{~d}, J=1.5 \mathrm{~Hz}, 1 \mathrm{H})$, $5.44(\mathrm{~d}, J=15.8 \mathrm{~Hz}, 1 \mathrm{H}), 4.30(\mathrm{~d}, J=15.7 \mathrm{~Hz}, 1 \mathrm{H})$.

${ }^{13}$ C NMR (100 MHz, DMSO-d $): \delta$ 153.7, 136.2, 134.0, 128.7, 128.2, 125.8, 124.9, 122.2, $121.3,119.3,117.4,113.9,113.6,113.4,109.6,56.0,44.7$.

Accurate mass (ESI, $\boldsymbol{m} / \mathbf{z})$ : calc'd for $\mathrm{C}_{17} \mathrm{H}_{14} \mathrm{~N}_{3} \mathrm{O}\left([\mathrm{M}+\mathrm{H}]^{+}\right)$: 276.1137, found $\mathrm{m} / z$ 276.1140.

TLC ( $\left(\mathbf{S i O}_{2}\right): R_{\mathrm{f}}=0.21(25 \%$ EtOAc in $n$-pentane) 


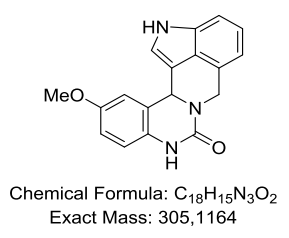

12-Methoxy-2,6,9,13b-tetrahydro-8H-pyrrolo $\left[4^{\prime}, 3^{\prime}, 2^{\prime}: 4,5\right]$ isoquinolino[2,3-c]quinazolin8-one (31b)

Prepared following the general procedure (A) but with 40 min of heating, starting from aldehyde $\mathbf{1 b}(20 \mathrm{mg}, 112 \mu \mathrm{mol})$ and amine $\mathbf{8}(18 \mathrm{mg}, 123 \mu \mathrm{mol})$.

Physical state and yield: white solid (15 mg, $164 \mu \mathrm{mol}, 51 \%)$.

${ }^{1}$ H NMR (400 MHz, DMSO-d6): $\delta$ 11.09-10.75 (m, 1H), 9.19 (s, 1H), 7.24-7.18 (m, 1H), 7.12-7.06 (m, 1H), 7.05-7.01 (m, 1H), 6.89-6.84 (m, 2H), 6.79-6.75 (m, 1H), 6.75-6.72 (m, 1H), $6.16(\mathrm{~s}, 1 \mathrm{H}), 5.46(\mathrm{~d}, J=15.7 \mathrm{~Hz}, 1 \mathrm{H}), 4.31(\mathrm{~d}, J=15.7 \mathrm{~Hz}, 1 \mathrm{H}), 3.82(\mathrm{~d}, J=1.1 \mathrm{~Hz}$, $3 \mathrm{H})$.

${ }^{13}$ C NMR (100 MHz, DMSO-d6): $\delta$ 154.1, 153.7, 133.9, 129.7, 128.8, 124.9, 122.2, 120.3, $117.5,114.5,113.9,113.6,113.4,111.1,109.6,56.2,55.4,44.7$.

Accurate mass (ESI, $\boldsymbol{m} / \boldsymbol{z})$ : calc'd for $\mathrm{C}_{18} \mathrm{H}_{16} \mathrm{~N}_{3} \mathrm{O}_{2}\left([\mathrm{M}+\mathrm{H}]^{+}\right)$: 306.1243 , found $m / z$ 306.1257.

TLC (SiO2): $R_{\mathrm{f}}=0.16(50 \%$ EtOAc in $n$-pentane).

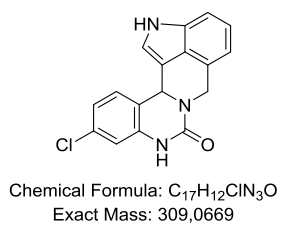

11-Chloro-2,6,9,13b-tetrahydro-8H-pyrrolo[ $\left[4^{\prime}, 3^{\prime}, 2^{\prime}: 4,5\right]$ isoquinolino[2,3-c]quinazolin-8one (31c)

Prepared following the general procedure (A) starting from aldehyde 1c (20 mg, $94 \mu \mathrm{mol})$ and amine 8 (18 mg, $123 \mu \mathrm{mol})$. 
Physical state and yield: yellow solid (25 mg, $81 \mu \mathrm{mol}, 86 \%)$.

${ }^{1}$ H NMR (400 MHz, DMSO-d6): $\delta 10.86$ (s, 1H), 9.56 (s, 1H), 7.52-7.46 (m, 1H), 7.27-7.21 (m, 1H), 7.15-7.07 (m, 2H), 6.94-6.85 (m, 2H), $6.73(\mathrm{~s}, 1 \mathrm{H}), 6.25(\mathrm{~s}, 1 \mathrm{H}), 5.46(\mathrm{~d}, J=15.8$ $\mathrm{Hz}, 1 \mathrm{H}), 4.37(\mathrm{~d}, J=15.8 \mathrm{~Hz}, 1 \mathrm{H})$.

${ }^{13}$ C NMR (100 MHz, DMSO-d6): $\delta$ 153.2, 137.8, 134.0, 132.4, 128.5, 127.7, 124.8, 122.3, $120.9,118.3,117.5,113.5,113.4,113.0,109.7,55.6,44.8$.

Accurate mass (ESI, $\boldsymbol{m} / \mathbf{z})$ : calc'd for $\mathrm{C}_{19} \mathrm{H}_{16} \mathrm{ClN}_{4} \mathrm{O}\left([\mathrm{M}+\mathrm{MeCN}+\mathrm{H}]^{+}\right): 351.1013$, found $\mathrm{m} / \mathrm{z}$ 351.1009 .

TLC (SiO $): R_{\mathrm{f}}=0.28(50 \%$ EtOAc in $n$-pentane).

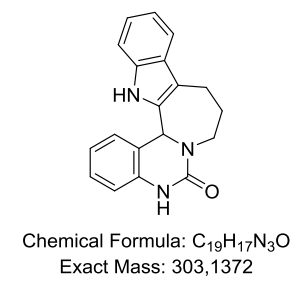

$5,8,9,10,15,15 b-H e x a h y d r o-6 H$-indolo[2',3' :3,4]azepino[1,2-c]quinazolin-6-one (32a)

Prepared following the general procedure (A) starting from aldehyde $1 \mathbf{a}(20 \mathrm{mg}, 112 \mu \mathrm{mol})$ and amine 9 (25 mg, $143 \mu \mathrm{mol})$.

Physical state and yield: yellow solid (32 mg, $106 \mu \mathrm{mol}, 94 \%)$.

${ }^{1}$ H NMR (400 MHz, DMSO-d6): 9.61 (s, 1H), 9.33 (s, 1H), 7.46-7.40 (m, 1H), 7.38-7.22 (m, 3H), 7.09-7.03 (m, 1H), 6.99-6.91 (m, 3H), $5.99(\mathrm{~s}, 1 \mathrm{H}), 4.69-4.30(\mathrm{~m}, 1 \mathrm{H}), 3.22-3.09$ (m, 1H), 3.09-2.91 (m, 1H), 2.93-2.73 (m, 1H), 1.96-1.83 (m, 2H).

${ }^{13}$ C NMR (100 MHz, DMSO-d $)$ : $\delta$ 153.6, 137.9, 136.8, 134.3, 128.8, 128.0, 126.6, 121.5, $120.4,118.5,118.4,117.5,113.9,112.0,111.8,56.7,48.5,27.2,22.1$.

Accurate mass (ESI, $\boldsymbol{m} / z)$ : calc'd for $\mathrm{C}_{19} \mathrm{H}_{18} \mathrm{~N}_{3} \mathrm{O}\left([\mathrm{M}+\mathrm{H}]^{+}\right)$: 304.1450, found $\mathrm{m} / z$ 304.1461. 
TLC (SiO $): R_{\mathrm{f}}=0.14(50 \%$ EtOAc in $n$-pentane)

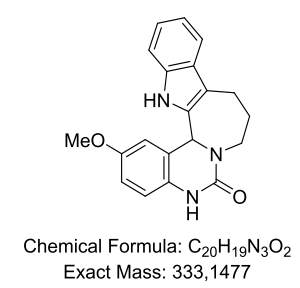

2-Methoxy-5,8,9,10,15,15b-hexahydro-6H-indolo[2',3' :3,4]azepino[1,2-c]quinazolin-6one (32b)

Prepared following the general procedure but with 40 min of heating, starting from aldehyde 1b $(20 \mathrm{mg}, 96 \mu \mathrm{mol})$ and amine $9(22 \mathrm{mg}, 126 \mu \mathrm{mol})$.

Physical state and yield: white solid (29 mg, $87 \mu \mathrm{mol}, 91 \%)$.

${ }^{1}$ H NMR (400 MHz, DMSO-d6): 9.71 (s, 1H), 9.18 (s, 1H), $7.54-7.42$ (m, 1H), 7.35-7.22 (m, 1H), 7.06-6.92 (m, 4H), $6.89(\mathrm{~d}, J=8.6 \mathrm{~Hz}, 1 \mathrm{H}), 5.98(\mathrm{~s}, 1 \mathrm{H}), 4.46(\mathrm{dt}, J=13.9,4.8 \mathrm{~Hz}$, $1 \mathrm{H}), 3.81(\mathrm{~s}, 3 \mathrm{H}), 3.21-3.14(\mathrm{~m}, 1 \mathrm{H}), 3.08-3.00(\mathrm{~m}, 1 \mathrm{H}), 2.91-2.81(\mathrm{~m}, 1 \mathrm{H}), 2.11-1.63(\mathrm{~m}$, 2H).

${ }^{13}$ C NMR (100 MHz, DMSO-d $)$ : $\delta$ 154.1, 153.8, 136.8, 134.3, 131.4, 128.0, 120.4, 119.3, $118.5,117.5,114.8,114.6,112.1,112.0,111.8,56.7,55.3,48.6,27.2,22.2$.

Accurate mass (ESI, $\boldsymbol{m} / z)$ : calc' $d$ for $\mathrm{C}_{20} \mathrm{H}_{20} \mathrm{~N}_{3} \mathrm{O}_{2}\left([\mathrm{M}+\mathrm{H}]^{+}\right)$: 334.1550 , found $m / z$ 334.1550.

TLC (SiO2): $R_{\mathrm{f}}=0.15$ (50\% EtOAc in $n$-pentane).

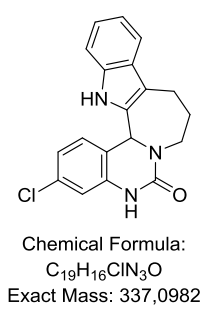




\section{3-Chloro-5,8,9,10,15,15b-hexahydro-6H-indolo[2',3':3,4]azepino[1,2-c]quinazolin-6-one}

(32c).

Prepared following the general procedure (A) starting from aldehyde 1c (20 mg, $94 \mu \mathrm{mol})$ and amine $9(21 \mathrm{mg}, 121 \mu \mathrm{mol})$.

Physical state and yield: white solid (30 mg, $89 \mu \mathrm{mol}, 95 \%)$.

${ }^{1}$ H NMR (400 MHz, DMSO-d f $_{\text {): }} 9.79$ (s, 1H), 9.54 (s, 1H), 7.50-7.45 (m, 1H), 7.41 (d, $J=$ 8.1 Hz, 1H), 7.31-7.24 (m, 1H), $7.15(\mathrm{dd}, J=8.1,2.1 \mathrm{~Hz}, 1 \mathrm{H}), 7.07-6.93(\mathrm{~m}, 3 \mathrm{H}), 6.08(\mathrm{~s}$, $1 \mathrm{H}), 4.45(\mathrm{dt}, J=13.9,4.4 \mathrm{~Hz}, 1 \mathrm{H}), 3.29-3.16(\mathrm{~m}, 1 \mathrm{H}), 3.12-2.99(\mathrm{~m}, 1 \mathrm{H}), 2.93-2.83(\mathrm{~m}$, $1 \mathrm{H}), 2.03-1.84(\mathrm{~m}, 2 \mathrm{H})$.

${ }^{13}$ C NMR (100 MHz, DMSO-d6): $\delta$ 153.1, 139.5, 136.6, 134.3, 133.1, 128.4, 128.0, 121.4, $120.6,118.6,117.6,117.3,113.5,112.2,111.7,56.0,48.9,27.1,22.3$.

Accurate mass (ESI, $\boldsymbol{m} / \boldsymbol{z})$ : calc'd for $\mathrm{C}_{21} \mathrm{H}_{20} \mathrm{ClN} 4 \mathrm{O}\left([\mathrm{M}+\mathrm{MeCN}+\mathrm{H}]^{+}\right): 379.1326$, found $\mathrm{m} / \mathrm{z}$ 379.1325 .

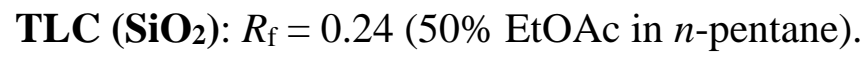

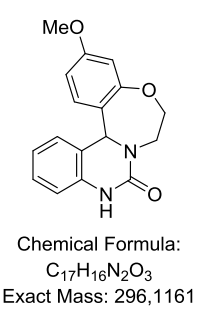

12-Methoxy-5,8,9,14b-tetrahydro-6H-benzo[6,7][1,4]oxazepino[4,5-c]quinazolin-6-one (33a)

Prepared following the general procedure but with 90 min of heating, starting from aldehyde $1 \mathrm{a}(20 \mathrm{mg}, 112 \mu \mathrm{mol})$ and amine $10(24 \mathrm{mg}, 144 \mu \mathrm{mol})$. Crystal structure available for download from the Cambridge Structural Database (CCDC 1473149).

Physical state and yield: white solid (24 mg, $81 \mu \mathrm{mol}, 72 \%)$. Single crystal for x-ray analysis prepared by vapour diffusion, $\mathrm{CHCl}_{3} /$ pentane. 
${ }^{1}$ H NMR (400 MHz, DMSO-d6): 9.47 (s, 1H), 7.36-7.22 (m, 1H), 7.22-7.12 (m, 1H), 7.06$6.99(\mathrm{~m}, 1 \mathrm{H}), 6.98-6.94(\mathrm{~m}, 1 \mathrm{H}), 6.65(\mathrm{~d}, J=2.6 \mathrm{~Hz}, 1 \mathrm{H}), 6.47(\mathrm{dd}, J=8.6,2.6 \mathrm{~Hz}, 1 \mathrm{H})$, $6.00(\mathrm{~d}, J=8.6 \mathrm{~Hz}, 1 \mathrm{H}), 5.81(\mathrm{~s}, 1 \mathrm{H}), 4.49-4.41(\mathrm{~m}, 1 \mathrm{H}), 4.36-4.28(\mathrm{~m}, 1 \mathrm{H}), 3.68(\mathrm{~s}, 3 \mathrm{H})$.

${ }^{13}$ C NMR (100 MHz, DMSO-d $)$ : $\delta$ 159.9, 159.7, 153.1, 137.9, 128.5, 127.6, 126.9, 126.8, $121.4,119.7,113.9,108.7,107.5,72.4,57.6,55.3,48.1$.

Accurate mass (ESI, $\boldsymbol{m} / z)$ : calc' $d$ for $\mathrm{C}_{17} \mathrm{H}_{16} \mathrm{~N}_{2} \mathrm{O}_{3}\left([\mathrm{M}+\mathrm{H}]^{+}\right)$: 297.1239, found $m / z$ 297.1246.

TLC (SiO2): $R_{\mathrm{f}}=0.11(50 \%$ EtOAc in $n$-pentane).

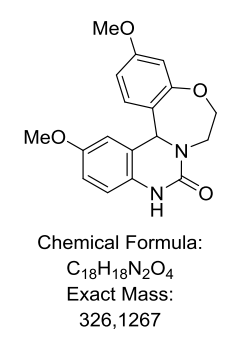

2,12-Dimethoxy-5,8,9,14b-tetrahydro-6H-benzo[6,7][1,4]oxazepino[4,5-c]quinazolin-6one (33b)

Prepared following the general procedure but with 40 min of heating, starting from aldehyde 1b $(20 \mathrm{mg}, 96 \mu \mathrm{mol})$ and amine $\mathbf{1 0}(24 \mathrm{mg}, 126 \mu \mathrm{mol})$.

Physical state and yield: white solid (14 mg, $43 \mu \mathrm{mol}, 45 \%)$.

${ }^{1} \mathbf{H}$ NMR (400 MHz, CDCl3): 7.77 (s, 1H), $6.89(\mathrm{dd}, J=8.7,2.7 \mathrm{~Hz}, 1 \mathrm{H}), 6.81(\mathrm{~d}, J=8.6 \mathrm{~Hz}$, $1 \mathrm{H}), 6.67(\mathrm{dd}, J=8.6,2.6 \mathrm{~Hz}, 2 \mathrm{H}), 6.42(\mathrm{dd}, J=8.7,2.6 \mathrm{~Hz}, 1 \mathrm{H}), 6.22(\mathrm{~d}, J=8.6 \mathrm{~Hz}, 1 \mathrm{H})$, $5.71(\mathrm{~s}, 1 \mathrm{H}), 4.59(\mathrm{dt}, J=14.5,1.8 \mathrm{~Hz}, 1 \mathrm{H}), 4.51-4.43(\mathrm{~m}, 1 \mathrm{H}), 3.89(\mathrm{ddd}, J=12.8,11.5,1.8$ $\mathrm{Hz}, 1 \mathrm{H}), 3.78$ (s, 3H), 3.75 (s, 3H), 3.40 (ddd, $J=14.5,11.5,2.7 \mathrm{~Hz}, 1 \mathrm{H})$.

${ }^{13}$ C NMR (100 MHz, CDCl 3$): \delta 160.5,160.3,155.3,154.2,131.1,128.3,126.8,120.7$, $115.3,114.9,111.9,109.1,107.9,73.2,59.3,55.8,55.6,49.1$.

Accurate mass (ESI, $\boldsymbol{m} / z)$ : calc'd for $\mathrm{C}_{20} \mathrm{H}_{22} \mathrm{~N}_{3} \mathrm{O}_{4}\left([\mathrm{M}+\mathrm{H}]^{+}\right)$: 368.1610 , found $m / z$ 368.1619. 
TLC (SiO $): R_{\mathrm{f}}=0.15(50 \%$ EtOAc in $n$-pentane)

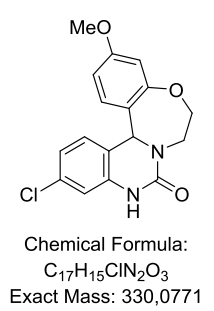

3-Chloro-12-methoxy-5,8,9,14b-tetrahydro-6H-benzo[6,7][1,4] oxazepino[4,5c]quinazolin-6-one (33c).

Prepared following the general procedure but with 40 min of heating, starting from aldehyde 1b $(20 \mathrm{mg}, 94 \mu \mathrm{mol})$ and amine $\mathbf{1 0}(20 \mathrm{mg}, 120 \mu \mathrm{mol})$.

Physical state and yield: white solid (19 mg, $58 \mu \mathrm{mol}, 61 \%)$.

${ }^{1}$ H NMR (400 MHz, DMSO-d6, one aliphatic signal obscured by solvent peak): $\delta 9.68$ (s, 1H), $7.26(\mathrm{~d}, J=8.1 \mathrm{~Hz}, 1 \mathrm{H}), 7.10(\mathrm{dd}, J=8.1,2.1 \mathrm{~Hz}, 1 \mathrm{H}), 7.03(\mathrm{~d}, J=2.1 \mathrm{~Hz}, 1 \mathrm{H}), 6.70(\mathrm{~d}$, $J=2.6 \mathrm{~Hz}, 1 \mathrm{H}), 6.53(\mathrm{dd}, J=8.6,2.6 \mathrm{~Hz}, 1 \mathrm{H}), 6.06(\mathrm{dd}, J=8.6,0.7 \mathrm{~Hz}, 1 \mathrm{H}), 5.90(\mathrm{~s}, 1 \mathrm{H})$, 4.67-4.22 (m, 1H), $3.72(\mathrm{~s}, 3 \mathrm{H}), 3.69-3.65(\mathrm{~m}, 1 \mathrm{H})$.

${ }^{13}$ C NMR (100 MHz, DMSO-d $\left.\mathbf{d}\right): \delta$ 159.9, 159.8, 152.7, 139.5, 132.8, 128.6, 127.5, 126.5, 121.2, 118.7, 113.4, 108.8, 107.7, 72.4, 57.1, 55.3, 48.1.

Accurate mass (ESI, $\boldsymbol{m} / \boldsymbol{z}$ ): calc' $\mathrm{d}$ for $\mathrm{C}_{19} \mathrm{H}_{19} \mathrm{ClN}_{3} \mathrm{O}_{4}\left([\mathrm{M}+\mathrm{MeCN}+\mathrm{H}]^{+}\right): 372.1115$, found $\mathrm{m} / \mathrm{z}$ 372.1123 .

TLC (SiO2): $R_{\mathrm{f}}=0.23$ (50\% EtOAc in $n$-pentane).

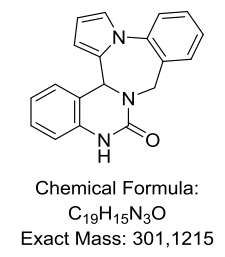




\section{5,16b-Dihydro-6H,8H-benzo[5,6]pyrrolo[2',1':3,4][1,4]diazepino[1,2-c]quinazolin-6-one}

(34a)

Prepared following the general procedure (A) starting from aldehyde 1a (20 mg, $112 \mu \mathrm{mol})$ and amine 11 (25 mg, $145 \mu \mathrm{mol})$.

Physical state and yield: white solid (26 mg, $86 \mu \mathrm{mol}, 77 \%)$.

${ }^{1}$ H NMR (400 MHz, DMSO-d6): $\delta 9.56$ (s, 1H), 7.72-7.48 (m, 3H), 7.43-7.33 (m, 1H), 7.31-7.25 (m, 1H), 7.24-7.16 (m, 1H), 7.02-6.94 (m, 1H), 6.89-6.80 (m, 2H), 6.31-6.11 (m, 1H), 5.60-5.53 (m, 1H), $5.49(\mathrm{~s}, 1 \mathrm{H}), 4.75(\mathrm{~d}, J=13.8 \mathrm{~Hz}, 1 \mathrm{H}), 3.97(\mathrm{~d}, J=13.8 \mathrm{~Hz}, 1 \mathrm{H})$.

${ }^{13}$ C NMR (100 MHz, DMSO-d6): $\delta$ 152.3, 139.6, 137.2, 133.0, 131.5, 130.0, 128.7, 127.8, $127.4,126.9,122.7,120.9,120.7,116.8,113.5,109.1,107.0,52.6,47.7$.

Accurate mass (ESI, $\boldsymbol{m} / z)$ : calc'd for $\mathrm{C}_{19} \mathrm{H}_{15} \mathrm{~N}_{3} \mathrm{O}\left([\mathrm{M}+\mathrm{H}]^{+}\right)$: 302.1293, found $\mathrm{m} / z$ 302.1290.

TLC (SiO2): $R_{\mathrm{f}}=0.26(50 \%$ EtOAc in $n$-pentane).

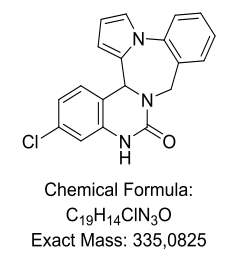

3-Chloro-5,16b-dihydro-6H,8H-benzo[5,6]pyrrolo[2',1':3,4][1,4]diazepino[1,2c]quinazolin-6-one (34b)

Prepared following the general procedure (A) starting from aldehyde 1c (20 mg, $94 \mu \mathrm{mol})$ and amine $11(21 \mathrm{mg}, 122 \mu \mathrm{mol})$.

Physical state and yield: yellow solid (22 mg, $66 \mu \mathrm{mol}, 70 \%)$.

${ }^{1}$ H NMR (400 MHz, DMSO-d6): $\delta 9.78$ (s, 1H), 7.71-7.58 (m, 3H), 7.48-7.40 (m, 1H), 7.38-7.26 (m, 1H), 7.14-7.00 (m, 1H), $7.0-6.89(\mathrm{~m}, 2 \mathrm{H}), 6.29-6.13(\mathrm{~m}, 1 \mathrm{H})$, 5.68-5.62 (m, 1H), $5.57(\mathrm{~s}, 1 \mathrm{H}), 4.80(\mathrm{~d}, J=13.9 \mathrm{~Hz}, 1 \mathrm{H}), 4.01(\mathrm{~d}, J=13.8 \mathrm{~Hz}, 1 \mathrm{H})$. 
${ }^{13}$ C NMR (100 MHz, DMSO-d $)$ : $\delta$ 151.7, 139.6, 138.7, 132.8, 132.6, 131.5, 130.1, 129.4, 127.6, 126.9, 122.8, 120.9, 120.7, 115.8, 112.9, 109.2, 107.0, 52.1, 47.7.

Accurate mass (ESI, $\boldsymbol{m} / z)$ : calc'd for $\mathrm{C}_{21} \mathrm{H}_{18} \mathrm{ClN} \mathrm{N}_{4} \mathrm{O}\left([\mathrm{M}+\mathrm{MeCN}+\mathrm{H}]^{+}\right): 377.1169$, found $m / z$ 377.1188 .

TLC $\left(\mathrm{SiO}_{2}\right): R_{\mathrm{f}}=0.30(50 \%$ EtOAc in $n$-pentane $)$.

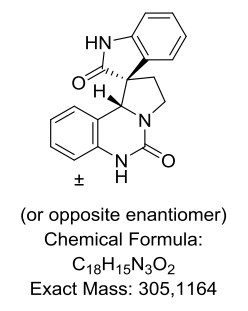

(syn)-2',3',6',10b'-Tetrahydro-5' $H$-spiro[indoline-3,1'-pyrrolo[1,2-c]quinazoline]-2,5'dione (35a, minor isomer)

Prepared following the general procedure (B) but with heating at $130^{\circ} \mathrm{C}$ for $20 \mathrm{~min}$, starting from aldehyde 1a $(20 \mathrm{mg}, 112 \mu \mathrm{mol})$ and amine 12 (hydrochloride salt $31 \mathrm{mg}, 146 \mu \mathrm{mol})$. A diastereomeric mixture (35a/35a') was obtained, data shown is for minor (isolated) diastereomer.

Physical state and yield: yellow solid ( $8 \mathrm{mg}, 26 \mu \mathrm{mol}, 23 \%)$.

${ }^{1}$ H NMR (400 MHz, DMSO-d6): $\delta 10.31$ (s, 1H), 9.25 (s, 1H), 7.64-7.52 (m, 1H), 7.36-7.27 (m, 1H), 7.17-7.12 (m, 1H), 7.11-7.05 (m, 1H), 6.93-6.85 (m, 1H), 6.80-6.74 (m, 1H), 6.62$6.55(\mathrm{~m}, 1 \mathrm{H}), 6.17-5.95(\mathrm{~m}, 1 \mathrm{H}), 5.27(\mathrm{~s}, 1 \mathrm{H}), 4.22-4.03(\mathrm{~m}, 1 \mathrm{H}), 3.63-3.51(\mathrm{~m}, 2 \mathrm{H}), 2.47-$ $2.33(\mathrm{~m}, 1 \mathrm{H}), 2.27-2.18(\mathrm{~m}, 1 \mathrm{H})$.

${ }^{13}$ C NMR (100 MHz, DMSO-d6): $\delta$ 178.7, 151.9, 142.7, 137.7, 129.9, 128.9, 128.7, 123.5, $123.4,122.2,120.7,116.6,113.9,109.7,65.0,57.3,43.8,33.1$.

Accurate mass (ESI, $\boldsymbol{m} / \boldsymbol{z})$ : calc'd for $\mathrm{C}_{18} \mathrm{H}_{16} \mathrm{~N}_{3} \mathrm{O}_{2}\left([\mathrm{M}+\mathrm{H}]^{+}\right)$: 306.1243 , found $m / z$ 306.1254. 
TLC (SiO $): R_{\mathrm{f}}=0.02(60 \%$ EtOAc in $n$-pentane $)$.

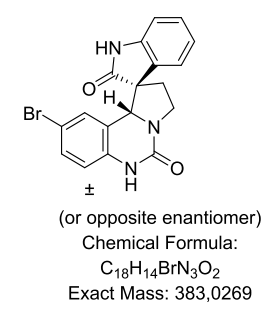

(syn)-9'-Bromo-2',3',6',10b'-tetrahydro-5' $H$-spiro[indoline-3,1'-pyrrolo[1,2-

c]quinazoline]-2,5'-dione (35b, minor isomer)

Prepared following the general procedure (B), starting from aldehyde 1d (20 mg, $77 \mu \mathrm{mol})$ and amine 12 (hydrochloride salt $21 \mathrm{mg}, 99 \mu \mathrm{mol}$ ). Silica gel chromatography was carried out using $3-5 \% \mathrm{MeOH}$ in DCM).

Physical state and yield: white solid (10 mg, $26 \mu \mathrm{mol}, 33 \%)$.

${ }^{1} \mathrm{H}$ NMR (400 MHz, CD3OD): $\delta$ 7.38-7.30 (m, 2H), 7.19-7.11 (m, 2H), 6.97-6.92 (m, 1H), $6.61(\mathrm{~d}, J=8.5 \mathrm{~Hz}, 1 \mathrm{H}), 6.30-5.89(\mathrm{~m}, 1 \mathrm{H}), 5.12(\mathrm{~s}, 1 \mathrm{H}), 4.26(\mathrm{dt}, J=11.4,8.6 \mathrm{~Hz}, 1 \mathrm{H}), 3.65$ (ddd, $J=11.4,9.1,3.9 \mathrm{~Hz}, 1 \mathrm{H}), 2.66-2.22(\mathrm{~m}, 2 \mathrm{H})$.

${ }^{13}$ C NMR (100 MHz, CD $\mathbf{3 O D ) : ~} \delta$ 180.2, 153.5, 136.4, 132.3, 129.9, 129.2, 127.3, 123.6, $123.2,118.7,116.3,114.1,110.9,65.8,58.5,44.5,33.2$.

Accurate mass (ESI, $\boldsymbol{m} / \boldsymbol{z}$ ): calc'd for $\mathrm{C}_{20} \mathrm{H}_{18} \mathrm{BrN}_{4} \mathrm{O}_{2}\left([\mathrm{M}+\mathrm{MeCN}+\mathrm{H}]^{+}\right): 425.0613$, found $\mathrm{m} / \mathrm{z}$ 425.0616 .

TLC (SiO) $): R_{\mathrm{f}}=0.13(8 \% \mathrm{MeOH}$ in DCM).

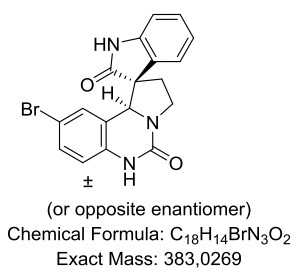


(anti)-9'-Bromo-2',3',6',10b'-tetrahydro-5' H-spiro[indoline-3,1'-pyrrolo[1,2c]quinazoline]-2,5'-dione (35b', major isomer)

Prepared following the general procedure (B), starting from aldehyde 1d (20 mg, $77 \mu \mathrm{mol})$ and amine 12 (hydrochloride salt, $21 \mathrm{mg}, 99 \mu \mathrm{mol}$ ). Silica gel chromatography was carried out using 3-5\% $\mathrm{MeOH}$ in DCM). Crystal structure available for download from the Cambridge Structural Database (CCDC 1473153).

Physical state and yield: white solid (13 mg, $34 \mu \mathrm{mol}, 43 \%)$. Single crystal for x-ray analysis prepared by vapour diffusion, THF/pentane.

${ }^{1} \mathrm{H}$ NMR (400 MHz, CD3OD): $\delta$ 7.13-7.08 (m, 1H), 7.07-7.03 (m, 1H), 6.90-6.86 (m, 1H), 6.84-6.83 (m, 1H), 6.83-6.82 (m, 1H), 6.77-6.74 (m, 1H), 6.61-6.47 (m, 1H), 4.14 (dt, $J=$ 11.8, $9.0 \mathrm{~Hz}, 1 \mathrm{H}), 3.74(\mathrm{ddd}, J=11.8,10.3,2.7 \mathrm{~Hz}, 1 \mathrm{H}), 2.57(\mathrm{ddd}, J=12.9,10.3,9.0 \mathrm{~Hz}$, $1 \mathrm{H}), 2.11(\mathrm{ddd}, J=12.9,8.6,2.7 \mathrm{~Hz}, 1 \mathrm{H})$.

${ }^{13}$ C NMR (100 MHz, CD3OD): $\delta$ 178.9, 153.2, 141.5, 136.0, 132.2, 130.7, 129.1, 127.4, $123.5,123.3,118.8,116.3,114.4,110.7,65.8,59.1,44.2,34.4$.

Accurate mass (ESI, $\boldsymbol{m} / \boldsymbol{z}$ ): calc'd for $\mathrm{C}_{20} \mathrm{H}_{18} \mathrm{BrN}_{4} \mathrm{O}_{2}\left([\mathrm{M}+\mathrm{MeCN}+\mathrm{H}]^{+}\right): 425.0613$, found $\mathrm{m} / z$ 425.0622 .

TLC (SiO) $): R_{\mathrm{f}}=0.23(8 \% \mathrm{MeOH}$ in DCM).

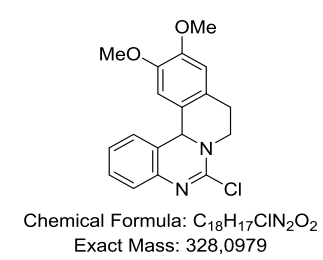

6-Chloro-11,12-dimethoxy-9,13b-dihydro-8H-isoquinolino[2,1-c]quinazoline (36a)

Compound 16a (200 mg, $0.64 \mathrm{mmol})$ in $\mathrm{POCl}_{3}(2 \mathrm{~mL})$ was heated at $110{ }^{\circ} \mathrm{C}$ for $4 \mathrm{~h}$. The volatiles were evaporated in vacuo and the residue was partitioned between saturated aqueous solution of $\mathrm{NaHCO}_{3}$ and DCM (20 mL of each). The aqueous layer was extracted into DCM 
$(2 \times 20 \mathrm{~mL})$ and the combined organics were washed with brine, dried over MgSO 4 and concentrated in vacuo. The residue was purified by silica gel chromatography (35\% EtOAc in $n$-pentane).

Physical state and yield: yellow solid (117 mg, $357 \mu \mathrm{mol}, 55 \%)$.

${ }^{1}$ H NMR (400 MHz, CDCl3): $\delta 7.30(\mathrm{td}, J=7.6,1.6 \mathrm{~Hz}, 1 \mathrm{H}), 7.22-7.15$ (m, 2H), 7.14-7.10 (m, 1H), $6.66(\mathrm{~s}, 1 \mathrm{H}), 6.53(\mathrm{~d}, J=0.7 \mathrm{~Hz}, 1 \mathrm{H}), 5.72(\mathrm{~s}, 1 \mathrm{H}), 4.40(\mathrm{ddd}, J=13.6,6.5,2.2 \mathrm{~Hz}$, 1H), $3.87(\mathrm{~s}, 3 \mathrm{H}), 3.70(\mathrm{~s}, 3 \mathrm{H}), 3.54(\mathrm{ddd}, J=13.6,11.7,5.1 \mathrm{~Hz}, 1 \mathrm{H}), 3.43-3.09(\mathrm{~m}, 1 \mathrm{H})$, $2.80(\mathrm{ddd}, J=16.4,5.1,2.1 \mathrm{~Hz}, 1 \mathrm{H})$.

${ }^{13}$ C NMR (100 MHz, CDCl 3$): \delta 148.6,148.5,147.4,142.4,129.0,127.6,126.4,126.0$, 125.0, 124.1, 122.2, 112.0, 109.1, 59.6, 56.0, 55.9, 47.7, 27.4.

Accurate mass (ESI, $\boldsymbol{m} / z)$ : calc'd for $\mathrm{C}_{18} \mathrm{H}_{18} \mathrm{ClN}_{2} \mathrm{O}_{2}\left([\mathrm{M}+\mathrm{H}]^{+}\right): 329.1057$, found $\mathrm{m} / z$ 329.1068.

TLC (SiO $): R_{\mathrm{f}}=0.36(50 \%$ EtOAc in $n$-pentane)

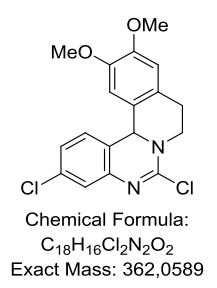

\section{3,6-Dichloro-11,12-dimethoxy-9,13b-dihydro-8H-isoquinolino[2,1-c]quinazoline (36b)}

Crude compound 16c (115 mg, prepared following general procedure A (but without purification) from aldehyde 1c, $70 \mathrm{mg}, 328 \mu \mathrm{mol})$ in $\mathrm{POCl}_{3}(2 \mathrm{~mL})$ was heated at $110{ }^{\circ} \mathrm{C}$ for 4 h. The volatiles were evaporated in vacuo and the residue was partitioned between saturated aqueous solution of $\mathrm{NaHCO}_{3}$ and DCM (20 mL of each). The aqueous layer was extracted into DCM (2 x $20 \mathrm{~mL})$ and the combined organics were washed with brine, dried over 
$\mathrm{MgSO} 4$ and concentrated in vacuo. The residue was purified by silica gel chromatography (40\% EtOAc in $n$-pentane).

Physical state and yield: yellow solid (80 mg, $221 \mu \mathrm{mol}, 66 \%)$.

${ }^{1}$ H NMR (400 MHz, CDCl3): $\delta$ 7.11-7.03 (m, 2H), 6.97-6.93 (m, 1H), 6.58 (s, 1H), 6.38 (d, $J=0.7 \mathrm{~Hz}, 1 \mathrm{H}), 5.64(\mathrm{~s}, 1 \mathrm{H}), 4.29$ (ddd, $J=13.6,6.4,2.5 \mathrm{~Hz}, 1 \mathrm{H}), 3.79(\mathrm{~s}, 3 \mathrm{H}), 3.64(\mathrm{~s}, 3 \mathrm{H})$, 3.47 (ddd, $J=13.6,11.4,5.1 \mathrm{~Hz}, 1 \mathrm{H}), 3.19-3.06(\mathrm{~m}, 1 \mathrm{H}), 2.74$ (ddd, $J=16.4,5.2,2.5 \mathrm{~Hz}$, $1 \mathrm{H})$.

${ }^{13}$ C NMR (100 MHz, CDCl3): $\delta$ 149.6, 148.7, 147.5, 142.6, 134.6, 127.5, 126.9, 126.0, $125.1,123.4,112.0,108.7,59.3,56.0,47.9,27.4$.

Accurate mass (ESI, m/z): calc'd for $\mathrm{C}_{18} \mathrm{H}_{17} \mathrm{ClN}_{2} \mathrm{O}_{2}\left([\mathrm{M}+\mathrm{H}]^{+}\right)$: 363.0667 , found $\mathrm{m} / \mathrm{z}$ 363.0670.

TLC (SiO $): R_{\mathrm{f}}=0.6(50 \%$ EtOAc in $n$-pentane)

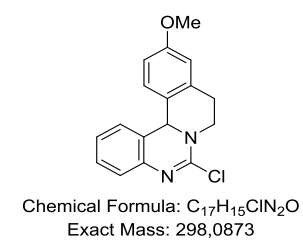

6-Chloro-11-methoxy-9,13b-dihydro-8H-isoquinolino[2,1-c]quinazoline (36c)

Compound $18(400 \mathrm{mg}, 1.4 \mathrm{mmol})$ in $\mathrm{POCl}_{3}(2 \mathrm{~mL})$ was heated at $110{ }^{\circ} \mathrm{C}$ for $4 \mathrm{~h}$. The volatiles were evaporated in vacuo and the residue was partitioned between saturated aqueous solution of $\mathrm{NaHCO}_{3}$ and DCM (20 mL of each). The aqueous layer was extracted into DCM (2 $\times 20 \mathrm{~mL}$ ) and the combined organics were washed with brine, dried over $\mathrm{MgSO}_{4}$ and concentrated in vacuo. The residue was purified by silica gel chromatography (30-35\% EtOAc in $n$-pentane).

Physical state and yield: yellow solid (235 mg, $0.79 \mathrm{mmol}, 55 \%)$. 
${ }^{1}$ H NMR (400 MHz, CDCl $)$ ) $\delta$ 7.22-7.16 (m, 1H), 7.11-7.05 (m, 2H), 7.01-6.98 (m, 1H), $6.81(\mathrm{dd}, J=8.5,0.9 \mathrm{~Hz}, 1 \mathrm{H}), 6.68-6.48(\mathrm{~m}, 3 \mathrm{H}), 5.64(\mathrm{~s}, 1 \mathrm{H}), 4.25(\mathrm{ddd}, J=13.6,6.6,2.6$ Hz, 1H), 3.68 (s, 3H), 3.45 (ddd, $J=13.6,11.3,5.3 \mathrm{~Hz}, 1 \mathrm{H}), 3.24-3.07$ (m, 1H), 2.77 (ddd, $J$ $=16.6,5.3,2.6 \mathrm{~Hz}, 1 \mathrm{H})$.

${ }^{13}$ C NMR (100 MHz, CDCl 3$): \delta 158.9,148.6,142.0,135.3,129.0,128.1,127.0,126.9$, $125.2,123.9,122.0,114.3,112.1,59.6,55.3,47.6,28.1$.

Accurate mass (ESI, m/z): calc'd for $\mathrm{C}_{17} \mathrm{H}_{16} \mathrm{ClN}_{2} \mathrm{O}\left([\mathrm{M}+\mathrm{H}]^{+}\right)$: 299.0951, found $\mathrm{m} / \mathrm{z}$ 299.0962.

TLC (SiO) $): R_{\mathrm{f}}=0.7$ (40\% EtOAc in $n$-pentane).

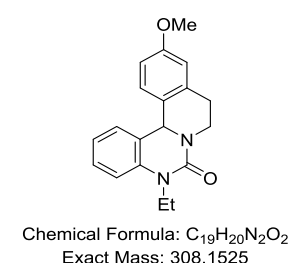

\section{5-Ethyl-11-methoxy-5,8,9,13b-tetrahydro-6H-isoquinolino[2,1-c]quinazolin-6-one (37)}

To a stirred solution of $\mathbf{1 8}(47 \mathrm{mg}, 168 \mu \mathrm{mol})$ in DMF $(1 \mathrm{~mL})$ under nitrogen atmosphere at ambient temperature was added $\mathrm{NaH}$ (60\% dispersion in mineral oil, $13 \mathrm{mg}, 325 \mu \mathrm{mol})$. After $5 \mathrm{~min}$, EtI $(38 \mathrm{mg}, 247 \mu \mathrm{mol})$ was added and the resulting mixture stirred for $22 \mathrm{~h}$, after which the reaction mixture was partitioned between EtOAc $(10 \mathrm{~mL})$ and $\mathrm{H}_{2} \mathrm{O}(10 \mathrm{~mL})$. The aqueous phase was extracted into EtOAc $(3 \times 10 \mathrm{~mL})$ and the combined organics were washed with brine, dried over $\mathrm{MgSO}_{4}$ and concentrated in vacuo. The residue was purified by preparative TLC (30\% EtOAc in $n$-pentane).

Physical state and yield: white solid (36 mg, $116 \mu \mathrm{mol}, 70 \%)$.

${ }^{1}$ H NMR (400 MHz, CDCl3): $\delta$ 7.34-7.28 (m, 1H), 7.18-7.09 (m, 1H), $7.04(\mathrm{td}, \mathrm{J}=7.4,1.1$ $\mathrm{Hz}, 1 \mathrm{H}), 6.97-6.84(\mathrm{~m}, 2 \mathrm{H}), 6.74-6.61(\mathrm{~m}, 2 \mathrm{H}), 5.47(\mathrm{~s}, 1 \mathrm{H}), 4.61-4.44(\mathrm{~m}, 1 \mathrm{H}), 4.04(\mathrm{dd}, \mathrm{J}=$ 
14.4, 7.2 Hz, 1H), 3.78 (s, 3H), $3.73(\mathrm{dd}, \mathrm{J}=14.4,7.1 \mathrm{~Hz}, 1 \mathrm{H}), 3.38-3.00$ (m, 2H), 2.99-2.59 $(\mathrm{m}, 1 \mathrm{H}), 1.25(\mathrm{t}, \mathrm{J}=7.0 \mathrm{~Hz}, 3 \mathrm{H})$.

${ }^{13}$ C NMR (100 MHz, CDCl3): $\delta$ 158.8, 155.1, 138.5, 137.1, 128.6, 127.4, 127.3, 127.2, $122.6,121.4,114.3,113.3,111.9,57.0,55.4,42.7,38.2,27.7,12.8$.

Accurate mass (ESI, $\boldsymbol{m} / z)$ : calc'd for $\mathrm{C}_{19} \mathrm{H}_{21} \mathrm{~N}_{2} \mathrm{O}_{2}\left([\mathrm{M}+\mathrm{H}]^{+}\right)$: 309.1603 , found $m / z$ 309.1598.

TLC (SiO) $): R_{\mathrm{f}}=0.8$ (30\% EtOAc in $n$-pentane).

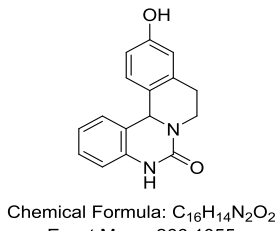

Exact Mass: 266,1055

\section{1-Hydroxy-5,8,9,13b-tetrahydro-6H-isoquinolino[2,1-c]quinazolin-6-one (38)}

To a stirred solution of $18(30 \mathrm{mg}, 107 \mu \mathrm{mol})$ in anhydrous DCM $(5 \mathrm{~mL})$ under nitrogen atmosphere at $-78{ }^{\circ} \mathrm{C}$ was added $\mathrm{BBr}_{3}(1 \mathrm{M}$ in hexanes, $0.2 \mathrm{~mL})$. The temperature was allowed to rise slowly to ambient temperature, after which the reaction mixture was stirred for $16 \mathrm{~h}$, after which the reaction mixture was slowly quenched with water. The reaction mixture was diluted with diethyl ether $(40 \mathrm{~mL})$ and $\mathrm{H}_{2} \mathrm{O}(15 \mathrm{~mL})$ and the precipitate was removed by filtration. The ether layer was extracted with $2 \mathrm{~N} \mathrm{NaOH}(30 \mathrm{~mL})$ and alkaline extract was then acidified with dil $\mathrm{HCl}$. The acidic extracts was extracted with diethyl ether (2 x $25 \mathrm{~mL})$ and the combined organics were washed with brine, dried over $\mathrm{MgSO}_{4}$ and concentrated in vacuo. The residue was used without further purification.

Physical state and yield: yellow solid (15 mg, $56 \mu \mathrm{mol}, 52 \%)$.

${ }^{1}$ H NMR (400 MHz, CD3OD): $\delta$ 7.38-7.16 (m, 2H), 7.05 (td, J = 7.5, 1.2 Hz, 1H), 6.85 (dd, J $=8.3,1.2 \mathrm{~Hz}, 1 \mathrm{H}), 6.73(\mathrm{dd}, \mathrm{J}=8.4,1.0 \mathrm{~Hz}, 1 \mathrm{H}), 6.61(\mathrm{~d}, \mathrm{~J}=2.5 \mathrm{~Hz}, 1 \mathrm{H}), 6.51(\mathrm{dd}, \mathrm{J}=8.4$, 
$2.5 \mathrm{~Hz}, 1 \mathrm{H}), 5.61(\mathrm{~s}, 1 \mathrm{H}), 4.36(\mathrm{ddd}, \mathrm{J}=13.0,6.7,2.9 \mathrm{~Hz}, 1 \mathrm{H}), 3.13(\mathrm{ddd}, \mathrm{J}=17.1,10.5,6.7$

$\mathrm{Hz}, 1 \mathrm{H}), 2.92-2.57(\mathrm{~m}, 2 \mathrm{H})$.

${ }^{13}$ C NMR (100 MHz, CD3OD): $\delta$ 157.7, 157.1, 138.2, 137.6, 129.6, 128.8, 128.5, 127.5, $122.8,121.0,116.6,115.2,113.9,58.9,43.2,28.1$.

Accurate mass (ESI, $\boldsymbol{m} / \boldsymbol{z}$ ): calc'd for $\mathrm{C}_{16} \mathrm{H}_{15} \mathrm{~N}_{2} \mathrm{O}_{2}\left([\mathrm{M}+\mathrm{H}]^{+}\right)$: 267.1134, found $m / z$ 267.1146.

TLC (SiO) $): R_{\mathrm{f}}=0.3$ (30\% EtOAc in $n$-pentane).

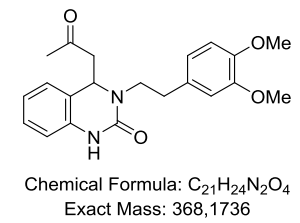

3-(3,4-Dimethoxyphenethyl)-4-(2-oxopropyl)-3,4-dihydroquinazolin-2(1H)-one (39a)

Prepared following the general procedure (D) starting from aldehyde $1 \mathbf{a}(20 \mathrm{mg}, 112 \mu \mathrm{mol})$, amine $\mathbf{2 a}(26 \mathrm{mg}, 143 \mu \mathrm{mol})$ and nucleophile $\mathbf{1 3}$ (64 mg, $1.1 \mathrm{mmol})$.

Physical state and yield: white solid (39 mg, $106 \mu \mathrm{mol}, 95 \%)$.

${ }^{1}$ H NMR (400 MHz, CDCl $): \delta 8.28$ (s, 1H), 7.20-7.13 (m, 1H), 6.96-6.85 (m, 2H), 6.81 $6.77(\mathrm{~m}, 1 \mathrm{H}), 6.74-6.70(\mathrm{~m}, 2 \mathrm{H}), 6.64-6.59(\mathrm{~m}, 1 \mathrm{H}), 4.65(\mathrm{t}, J=6.4 \mathrm{~Hz}, 1 \mathrm{H}), 4.22(\mathrm{dt}, J=$ 13.6, $6.7 \mathrm{~Hz}, 1 \mathrm{H}), 3.81(\mathrm{~s}, 3 \mathrm{H}), 3.62(\mathrm{~s}, 3 \mathrm{H}), 3.10(\mathrm{dt}, J=14.1,7.4 \mathrm{~Hz}, 1 \mathrm{H}), 2.93-2.72(\mathrm{~m}$, 4H), $1.99(\mathrm{~s}, 3 \mathrm{H})$.

${ }^{13}$ C NMR (100 MHz, CDCl 3$): \delta$ 206.2, 154.8, 148.7, 147.4, 136.5, 131.4, 128.3, 125.6, 122.1, 120.6, 113.9, 112.1, 111.3, 55.8, 55.5, 55.3, 48.5, 48.1, 34.4, 31.2.

Accurate mass (ESI, $\boldsymbol{m} / z)$ : calc'd for $\mathrm{C}_{21} \mathrm{H}_{25} \mathrm{~N}_{2} \mathrm{O}_{4}\left([\mathrm{M}+\mathrm{H}]^{+}\right)$: 369.1814 , found $m / z$ 369.1816.

TLC (SiO2): $R_{\mathrm{f}}=0.20(50 \%$ EtOAc in $n$-pentane) 


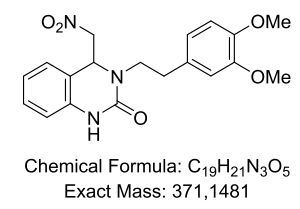

3-(3,4-Dimethoxyphenethyl)-4-(nitromethyl)-3,4-dihydroquinazolin-2(1H)-one (39b)

Prepared following the general procedure (D) starting from aldehyde $\mathbf{1 a}(20 \mathrm{mg}, 112 \mu \mathrm{mol})$, amine 2a (26 mg, $143 \mu \mathrm{mol})$ and nucleophile 14 (64 mg, $330 \mu \mathrm{mol})$.

Physical state and yield: white solid (38 mg, $102 \mu \mathrm{mol}, 90 \%)$.

${ }^{1}$ H NMR (400 MHz, CDCl3): $\delta 8.72(\mathrm{~s}, 1 \mathrm{H}), 7.33-7.19$ (m, 1H), 7.00-6.90 (m, 1H), 6.88 $6.78(\mathrm{~m}, 2 \mathrm{H}), 6.75-6.65(\mathrm{~m}, 2 \mathrm{H}), 6.54(\mathrm{~d}, J=1.7 \mathrm{~Hz}, 1 \mathrm{H}), 4.72(\mathrm{t}, J=6.7 \mathrm{~Hz}, 1 \mathrm{H}), 4.52(\mathrm{dd}$, $J=11.9,6.8 \mathrm{~Hz}, 1 \mathrm{H}), 4.44-4.19(\mathrm{~m}, 2 \mathrm{H}), 3.80(\mathrm{~s}, 3 \mathrm{H}), 3.57(\mathrm{~s}, 3 \mathrm{H}), 3.10(\mathrm{dt}, J=14.2,7.3 \mathrm{~Hz}$, $1 \mathrm{H}), 2.82(\mathrm{t}, J=6.8 \mathrm{~Hz}, 2 \mathrm{H})$.

${ }^{13}$ C NMR (100 MHz, CDCl 3$): \delta 154.2,148.8,147.6,136.8,131.0,129.7,125.5,122.7$, $120.7,117.2,114.4,112.0,111.4,58.7,55.9,55.5,49.1,48.0,34.6$.

Accurate mass (ESI, $\boldsymbol{m} / z)$ : calc'd for $\mathrm{C}_{19} \mathrm{H}_{22} \mathrm{~N}_{3} \mathrm{O}_{5}\left([\mathrm{M}+\mathrm{H}]^{+}\right)$: 372.1559 , found $m / z$ 372.1576.

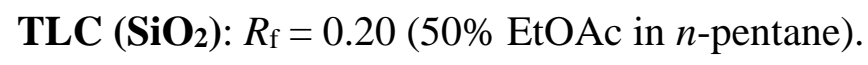

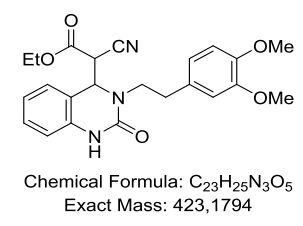

Ethyl 2-cyano-2-(3-(3,4-dimethoxyphenethyl)-2-oxo-1,2,3,4-tetrahydroquinazolin-4yl)acetate (39c)

Prepared following the general procedure (D) starting from aldehyde 1a (40 mg, $224 \mu \mathrm{mol}$ ), amine $\mathbf{2 a}(53 \mathrm{mg}, 292 \mu \mathrm{mol})$ and nucleophile $\mathbf{1 5}(51 \mathrm{mg}, 450 \mu \mathrm{mol})$.

Physical state and yield: white solid (67 mg, $158 \mu \mathrm{mol}, 71 \%)$. 
${ }^{1} \mathrm{H}$ NMR (400 MHz, CDCl3, 1.1:1 diastereomeric mixture, diagnostic peaks): $\delta 8.45$ (s, N$H$ ), $4.17\left(\mathrm{q}, J=7.2 \mathrm{~Hz}, 2 \mathrm{H}, \mathrm{CH}_{2}-\mathrm{CH}_{3}\right), 1.22\left(\mathrm{t}, \mathrm{J}=7.2 \mathrm{~Hz}, 1 \mathrm{H}, \mathrm{CH}_{2}-\mathrm{CH}_{3}\right)$.

${ }^{13}$ C NMR (100 MHz, CDCl3, data for major isomer): $\delta 164.2,153.9,148.9,147.7,137.0$, 131.1, 130.0, 126.0, 122.5, 120.7, 120.7, 114.6, 114.5, 112.1, 112.0, 111.5, 63.6, 61.3, 56.0, 49.6, 43.6, 34.5, 14.0 .

Accurate mass (ESI, $\boldsymbol{m} / z)$ : calc' $d$ for $\mathrm{C}_{23} \mathrm{H}_{26} \mathrm{~N}_{3} \mathrm{O}_{5}\left([\mathrm{M}+\mathrm{H}]^{+}\right)$: 424.1872, found $m / z$ 424.1880.

TLC (SiO $): R_{\mathrm{f}}=0.15(30 \%$ EtOAc in $n$-pentane)

\section{Conformational Analysis}

Conformational analysis was performed using Mixed torsional/Low-mode sampling in MacroModel, ${ }^{5}$ employing the OPLS3 force field ${ }^{6,7}$ and the Generalized-Born/Surface-Area (GB/SA) model for water as implemented in MacroModel. The analysis was set up with 2000 steps per searched torsion angle and Enhanced torsion sampling. For energy minimization a maximum of 2500 steps of Truncated Newton Conjugate Gradient was performed with a convergence criterion of $0.05 \mathrm{~kJ}$ mol- $1 \AA$ - 1 .
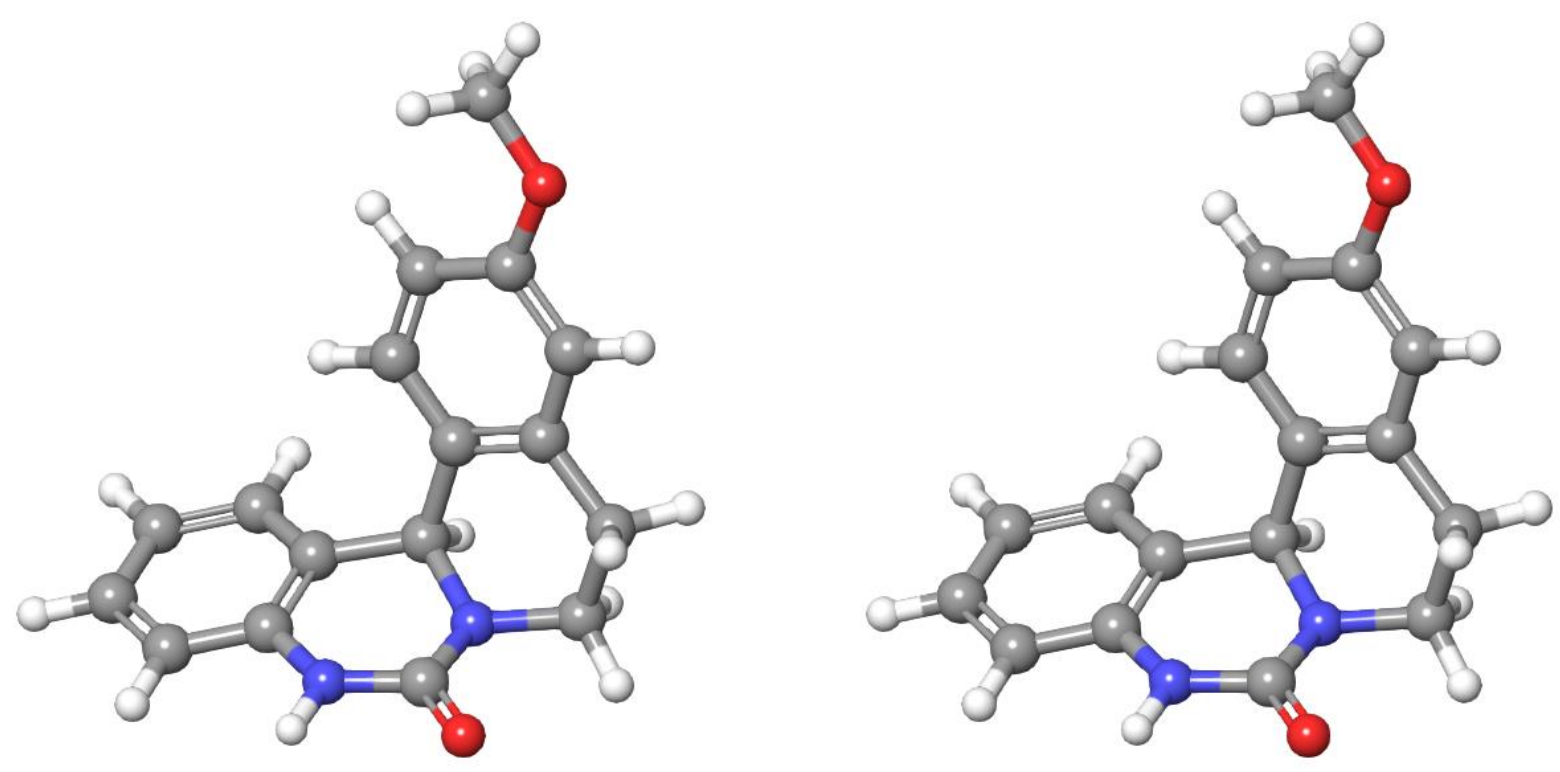

Figure S1. Lowest energy conformation found of $\mathbf{1 8}$ in cross-eyed stereo representation. 

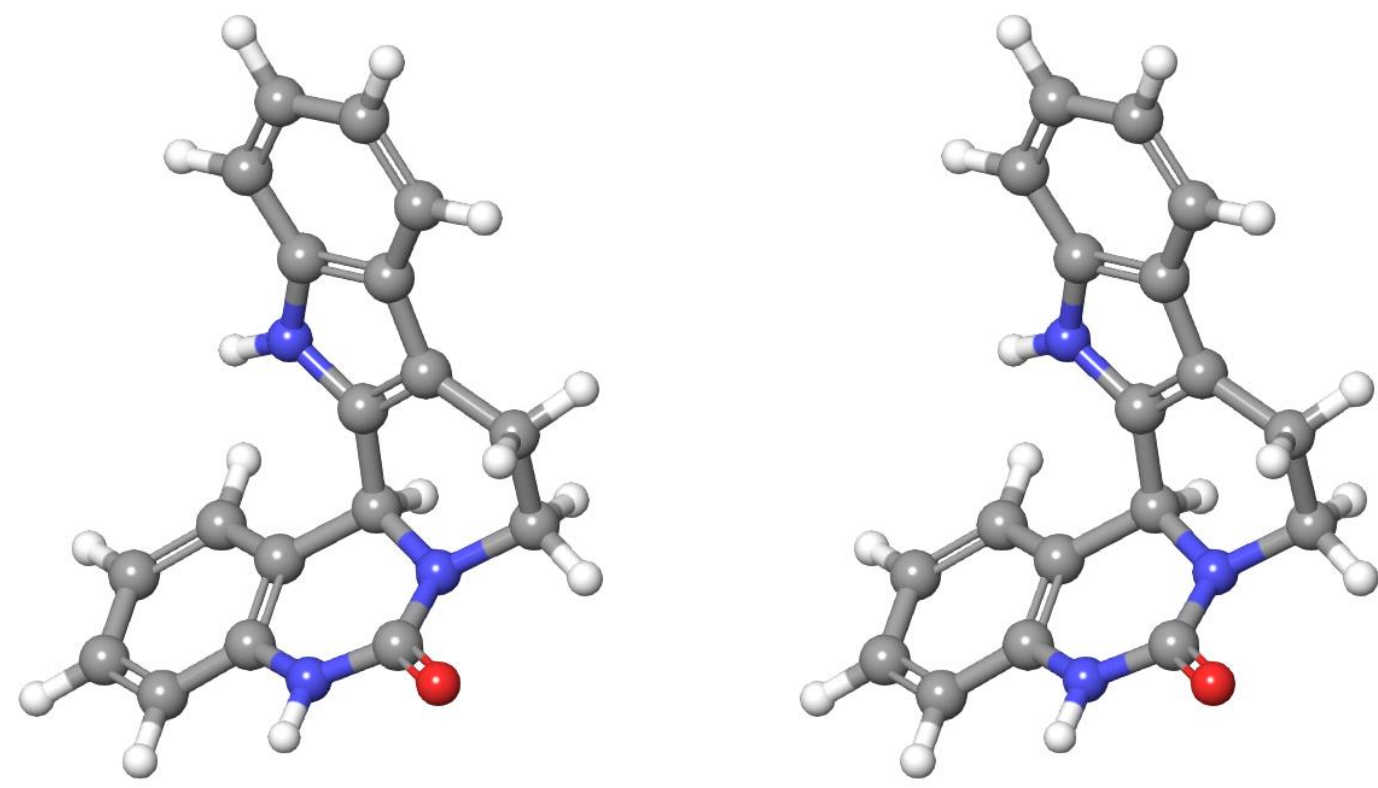

Figure S2. Lowest energy conformation found of 21a in cross-eyed stereo representation.
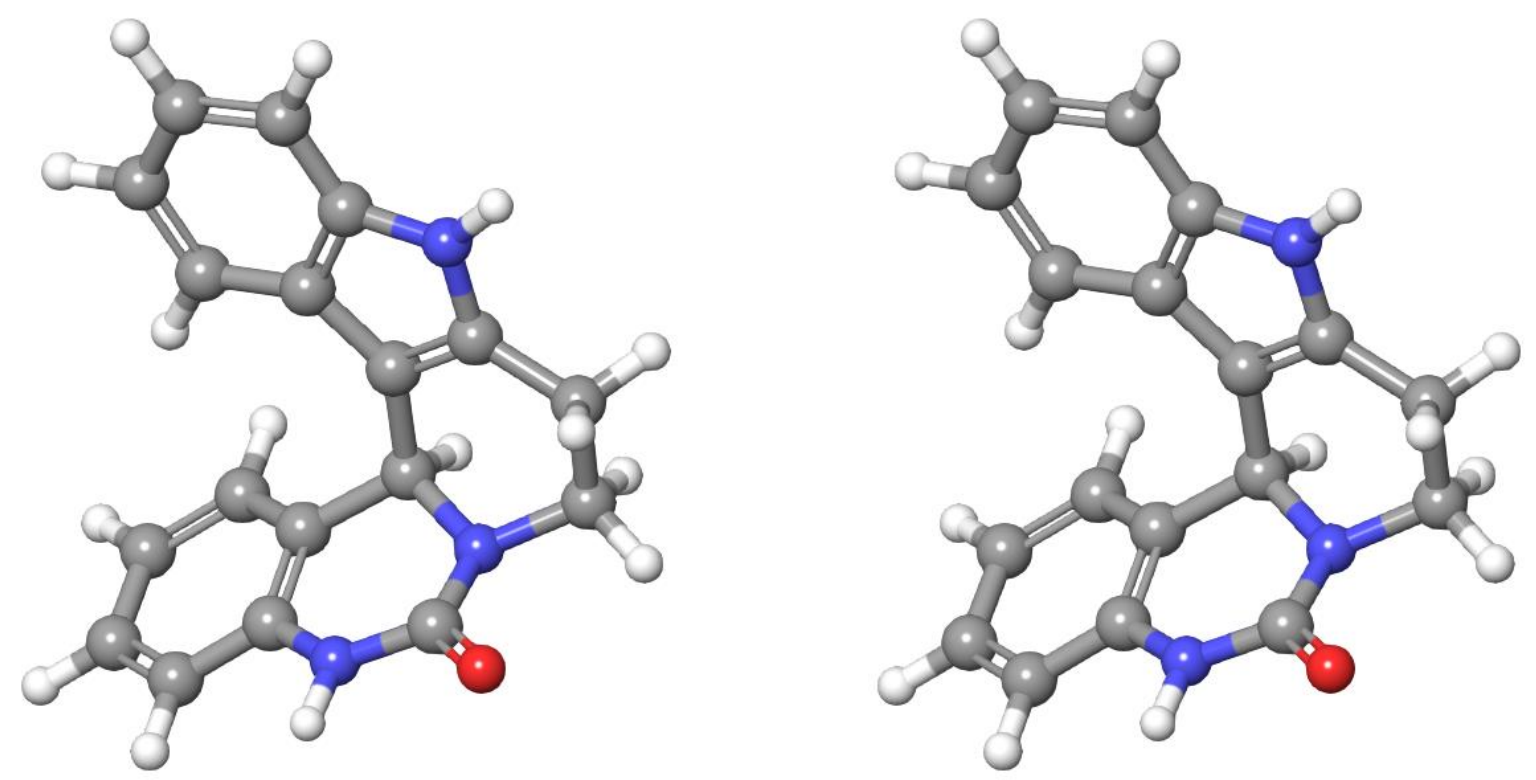

Figure S3. Lowest energy conformation found of $\mathbf{2 7 a}$ in cross-eyed stereo representation. 

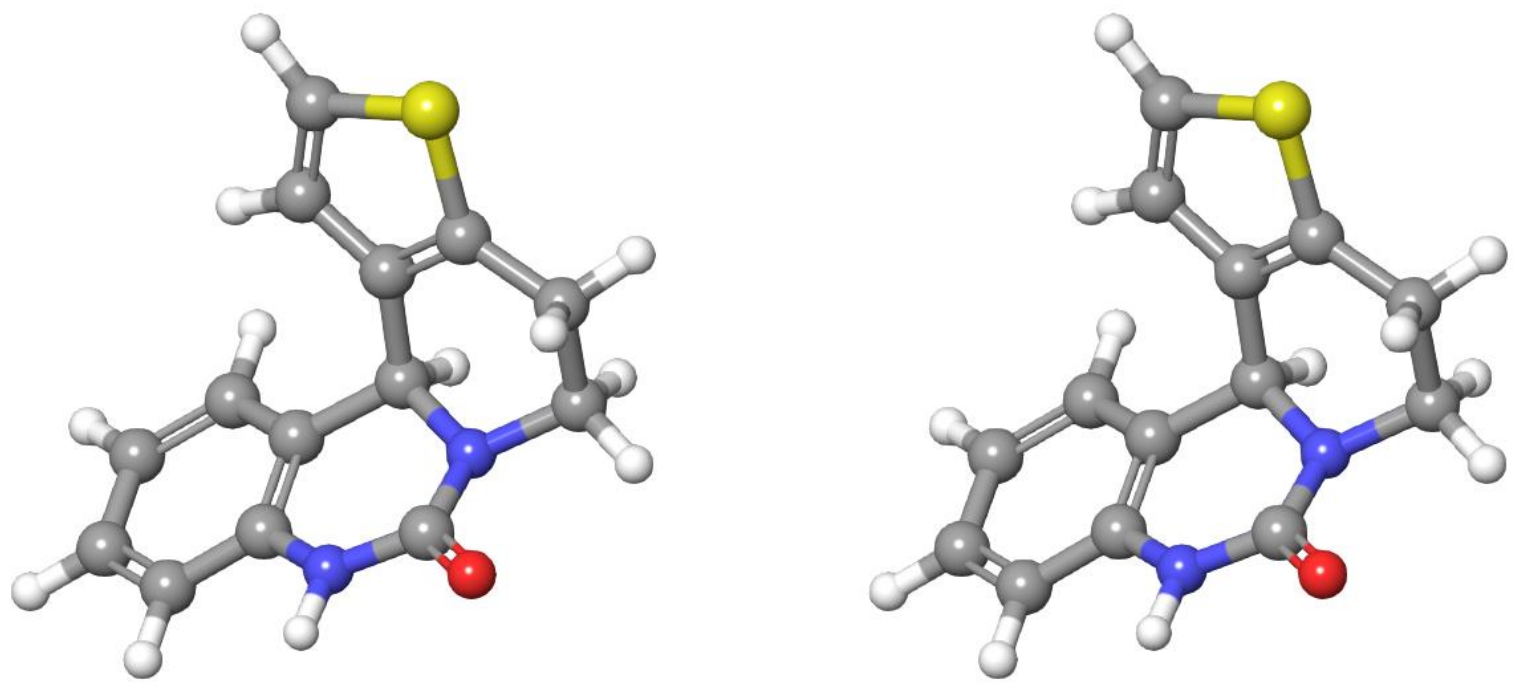

Figure S4. Lowest energy conformation found of $\mathbf{2 8 a}$ in cross-eyed stereo representation.
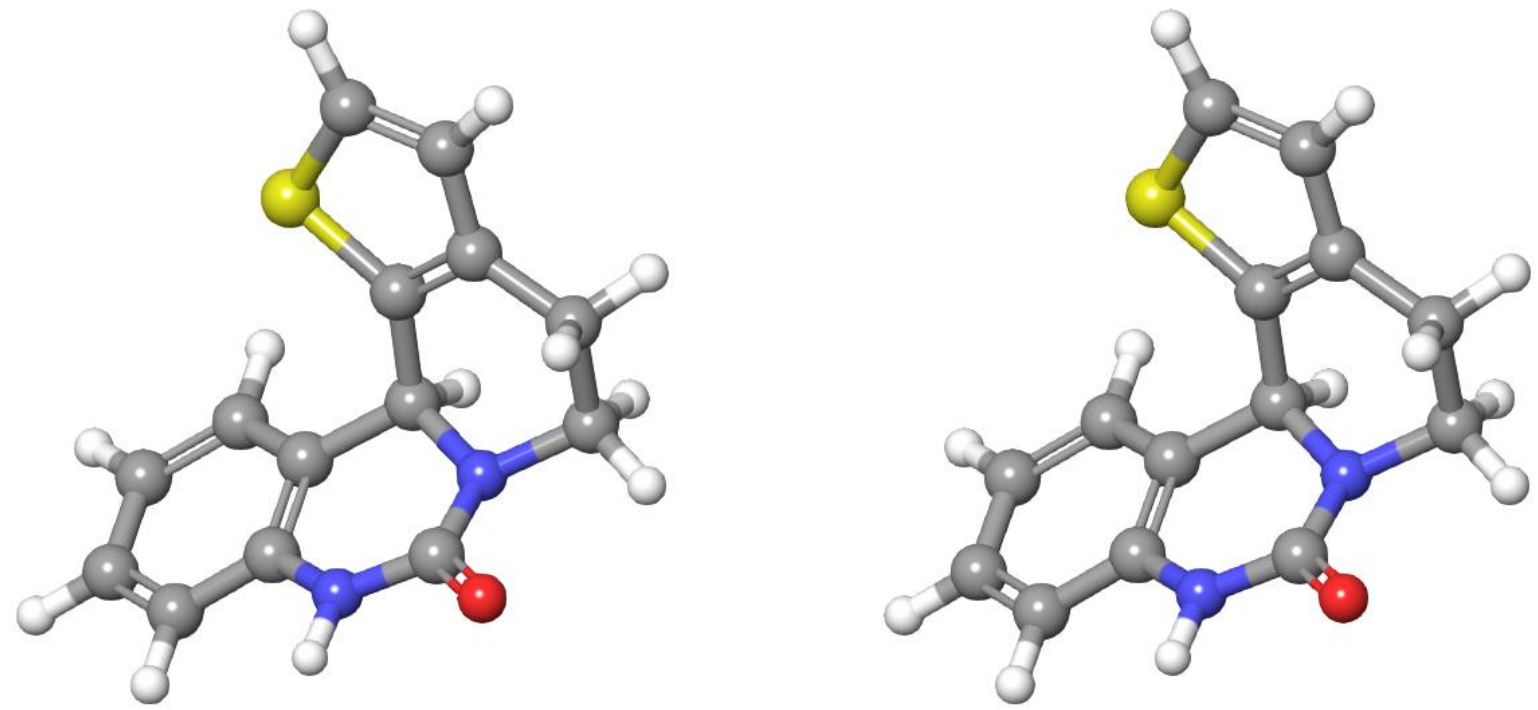

Figure S5. Lowest energy conformation found of 29a in cross-eyed stereo representation.
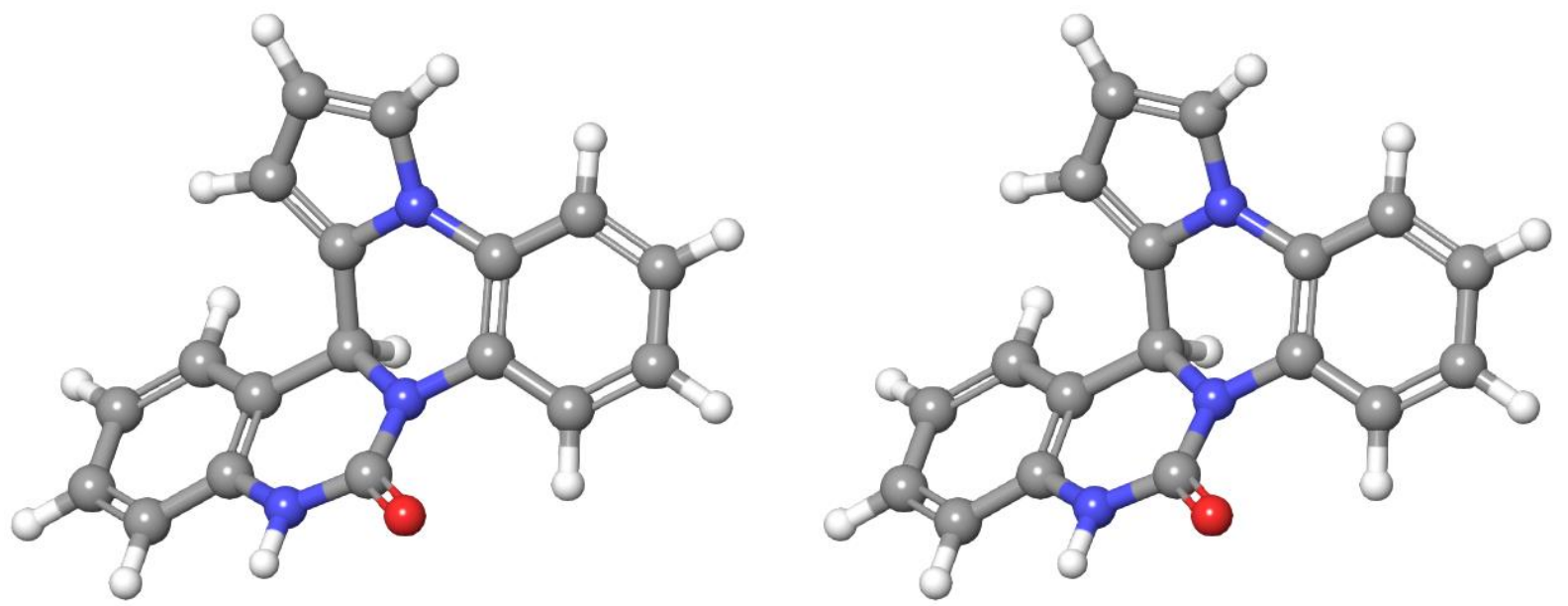
Figure S6. Lowest energy conformation found of $\mathbf{3 0 a}$ in cross-eyed stereo representation.
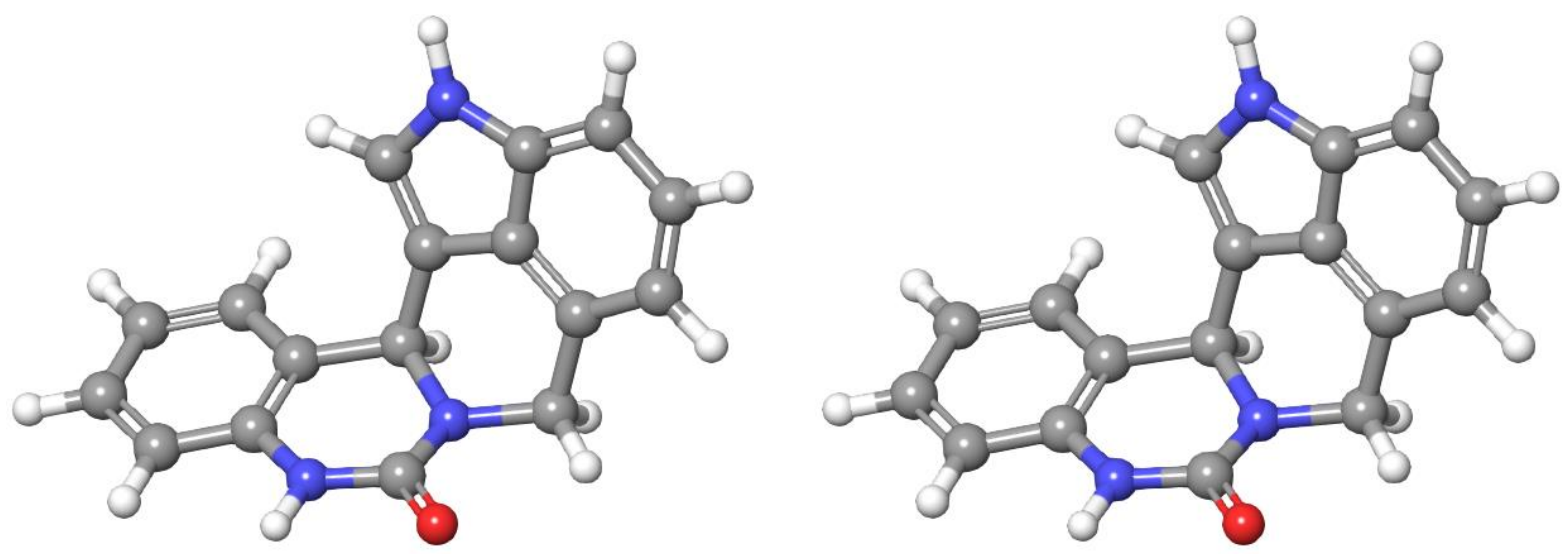

Figure S7. Lowest energy conformation found of 31a in cross-eyed stereo representation.
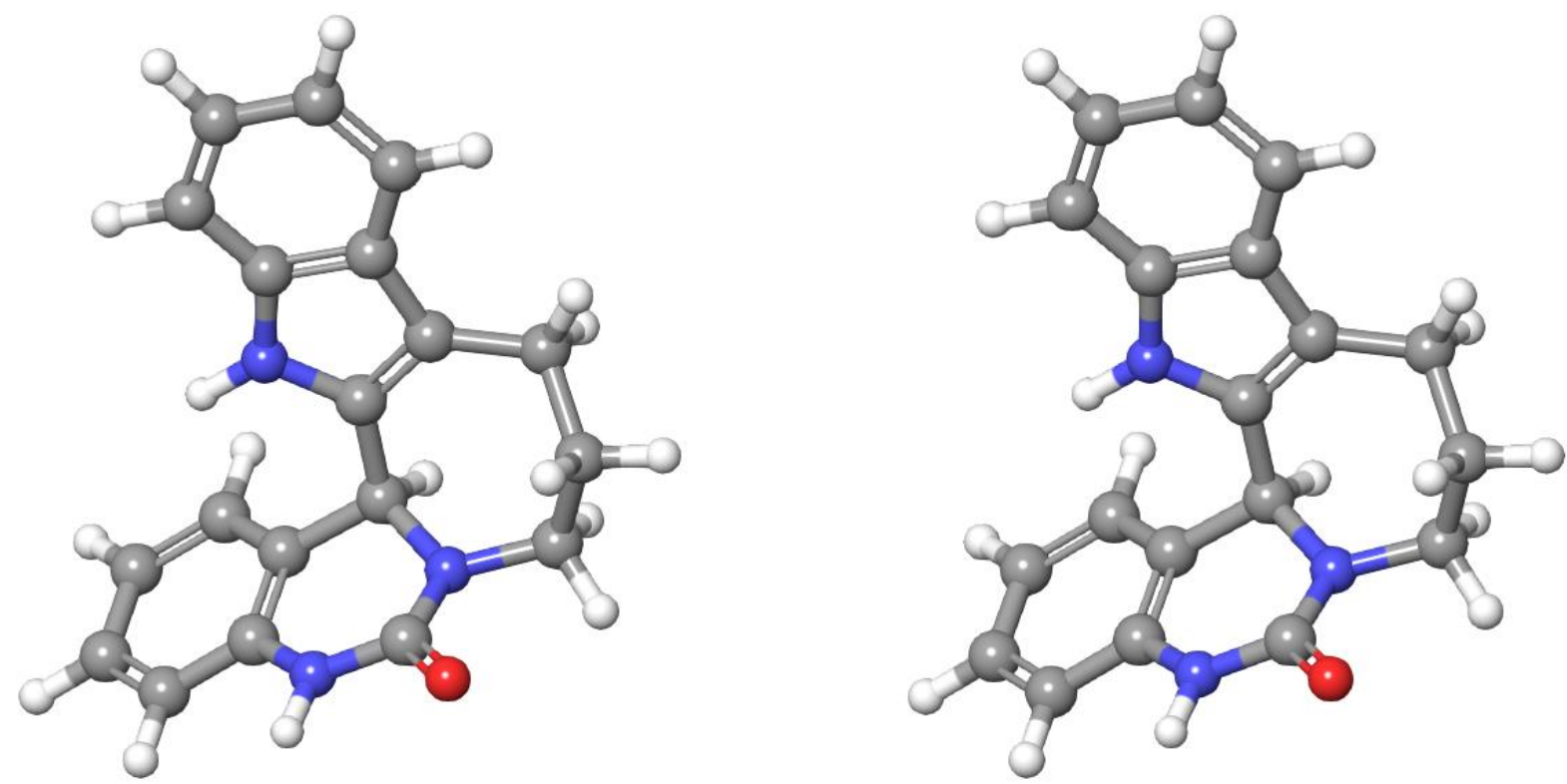

Figure S8. Lowest energy conformation found of 32a in cross-eyed stereo representation. 

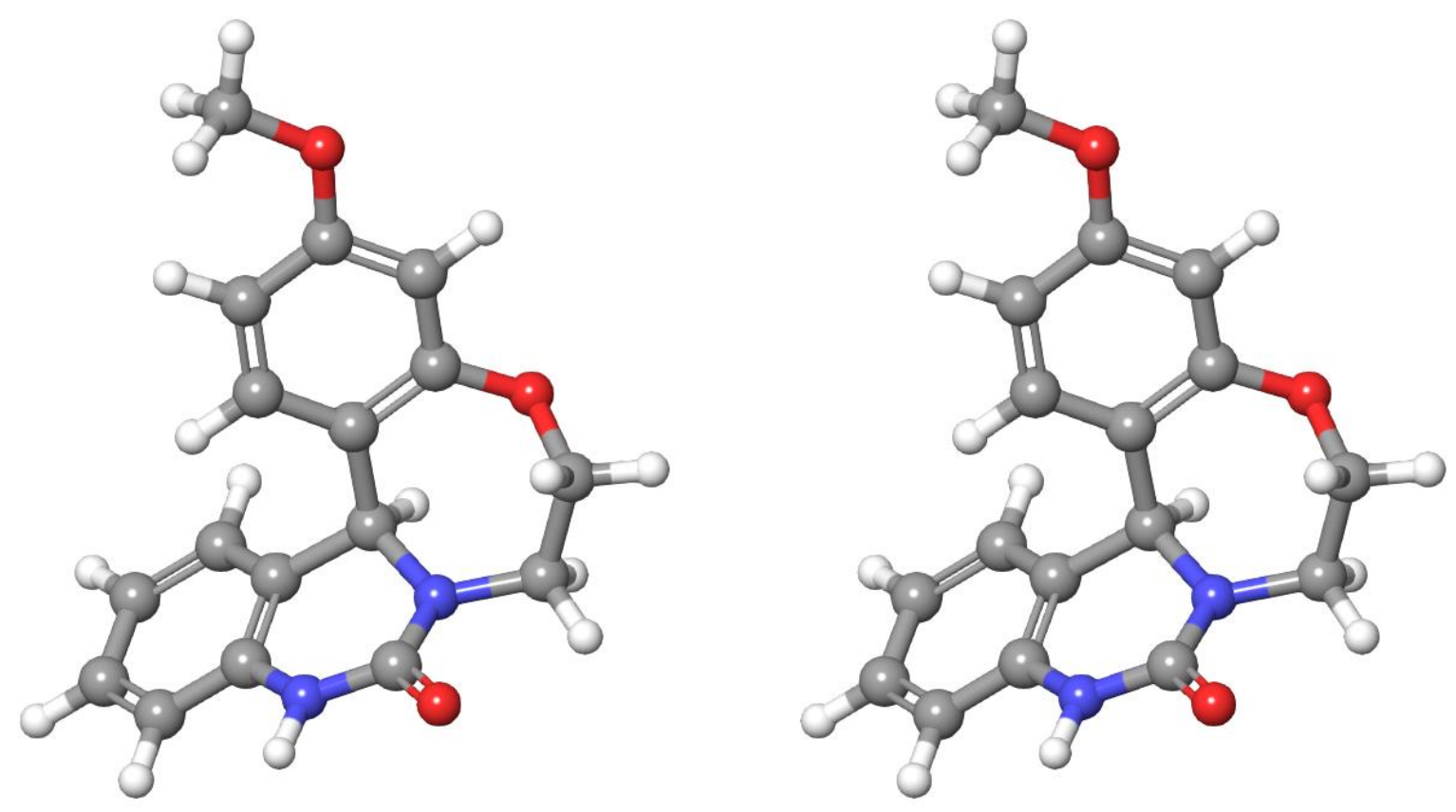

Figure S9. Lowest energy conformation found of 33a in cross-eyed stereo representation.
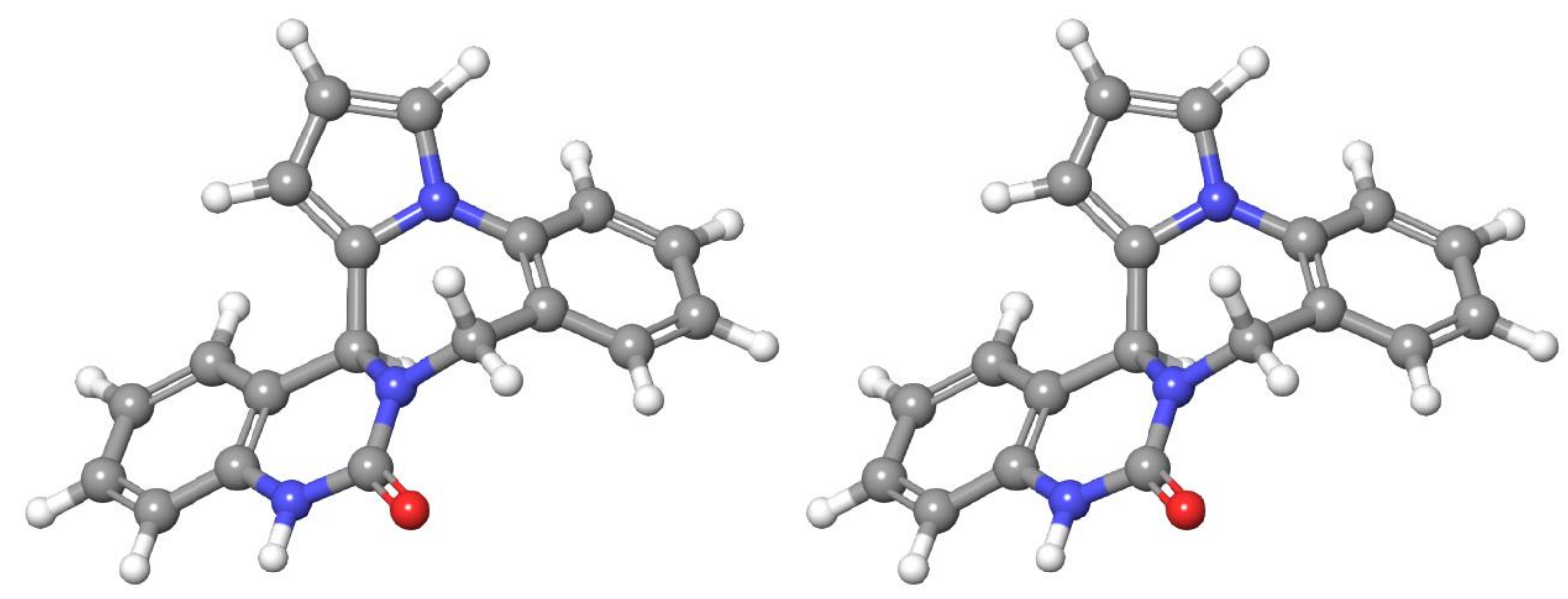

Figure S10. Lowest energy conformation found of 34a in cross-eyed stereo representation. 

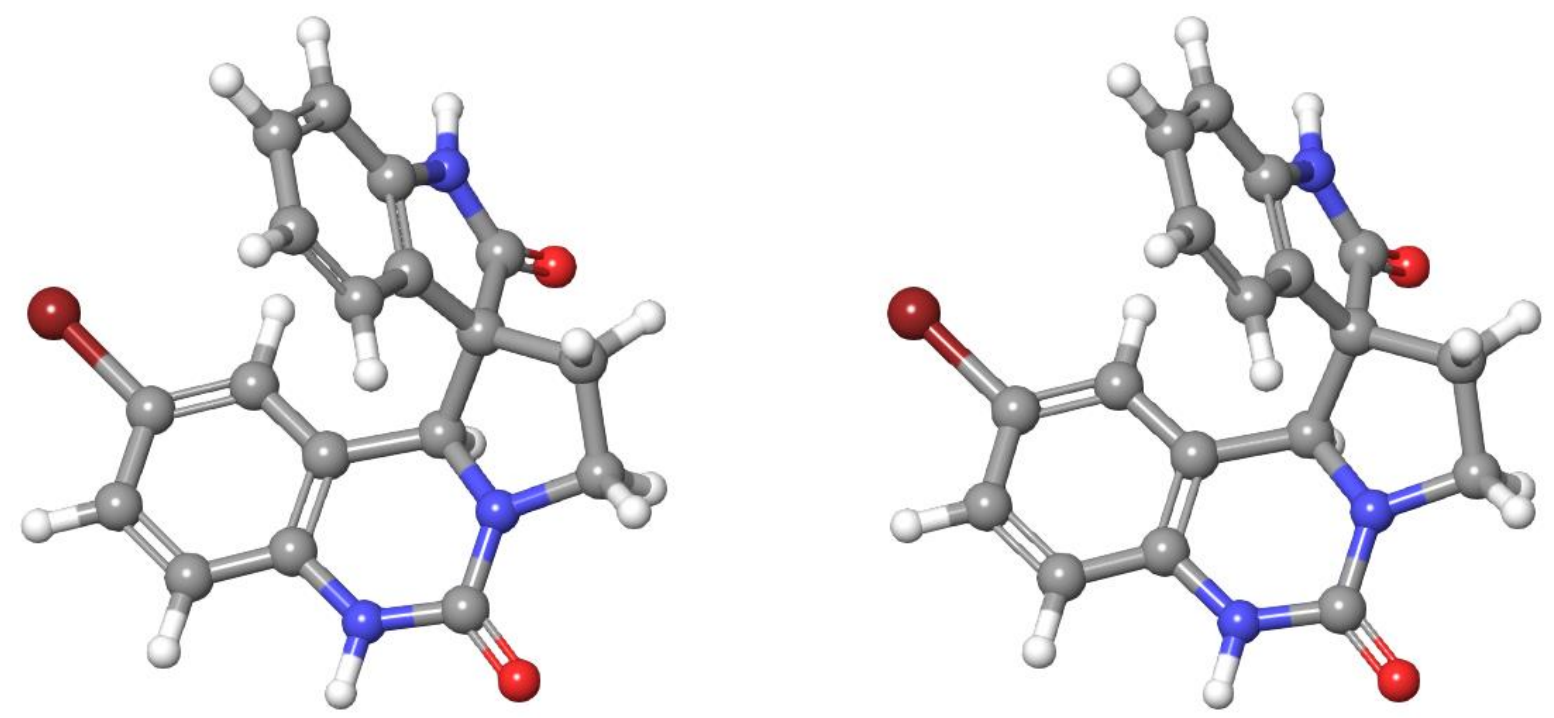

Figure S11. Lowest energy conformation found of $\mathbf{3 5 b}$ ' in cross-eyed stereo representation. 


\section{Literature references}

[1] M. Y. Stevens, K. Wieckowski, P. Wu, R. T. Sawant, L. R. Odell, Org. Biomol. Chem. 2015, 13, 2044-2054.

[2] S. Kano, Y. Yuasa, S. Shibuya, Synthesis 1984, 1984, 1071-1073.

[3] Commercially available (CAS 61894-74-4)

[4] O. N. Chupakhin, T. L. Pilicheva, I. Y. Postovskii, N. A. Klyuev, Khimiya Geterotsiklicheskikh Soedin. 1975, 708-712.

[5] Schrödinger Release 2016-2: MacroModel, version 11.2, Schrödinger, LLC, New York, NY, 2016.

[6] OPLS3, Schrödinger, Inc., New York, NY, 2013

[7] Shivakumar, D.; Harder, E; Damm, W.; Friesner, R.A.; Sherman, W. Improving the prediction of absolute solvation free energies using the Next Generation OPLS force field, J. Chem. Theory Comput. 2012, 8, 2553

\section{NMR spectra for all compounds}
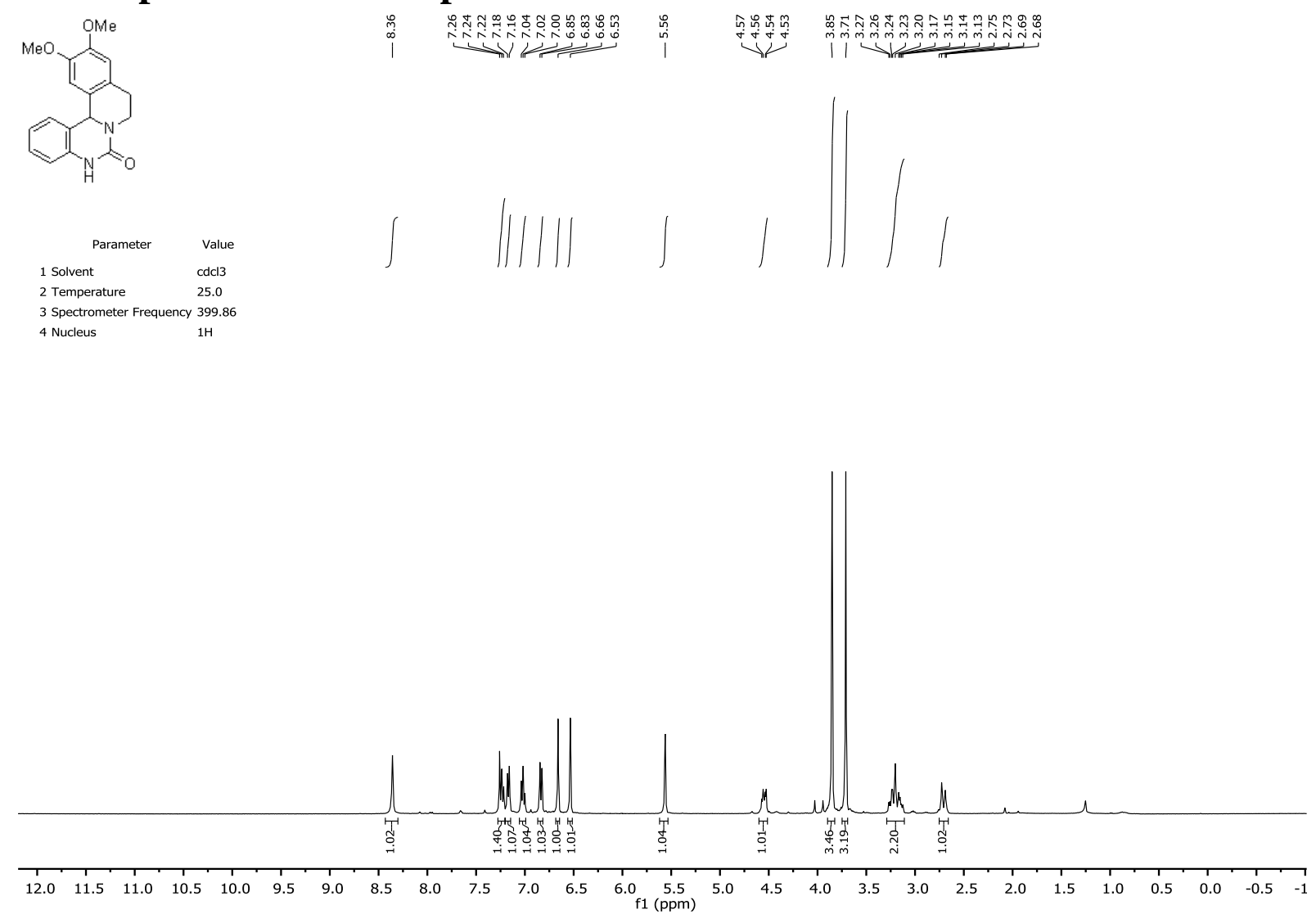


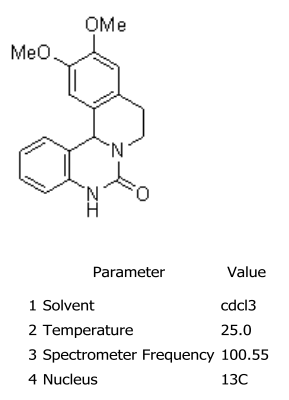

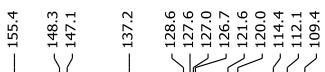

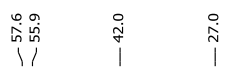

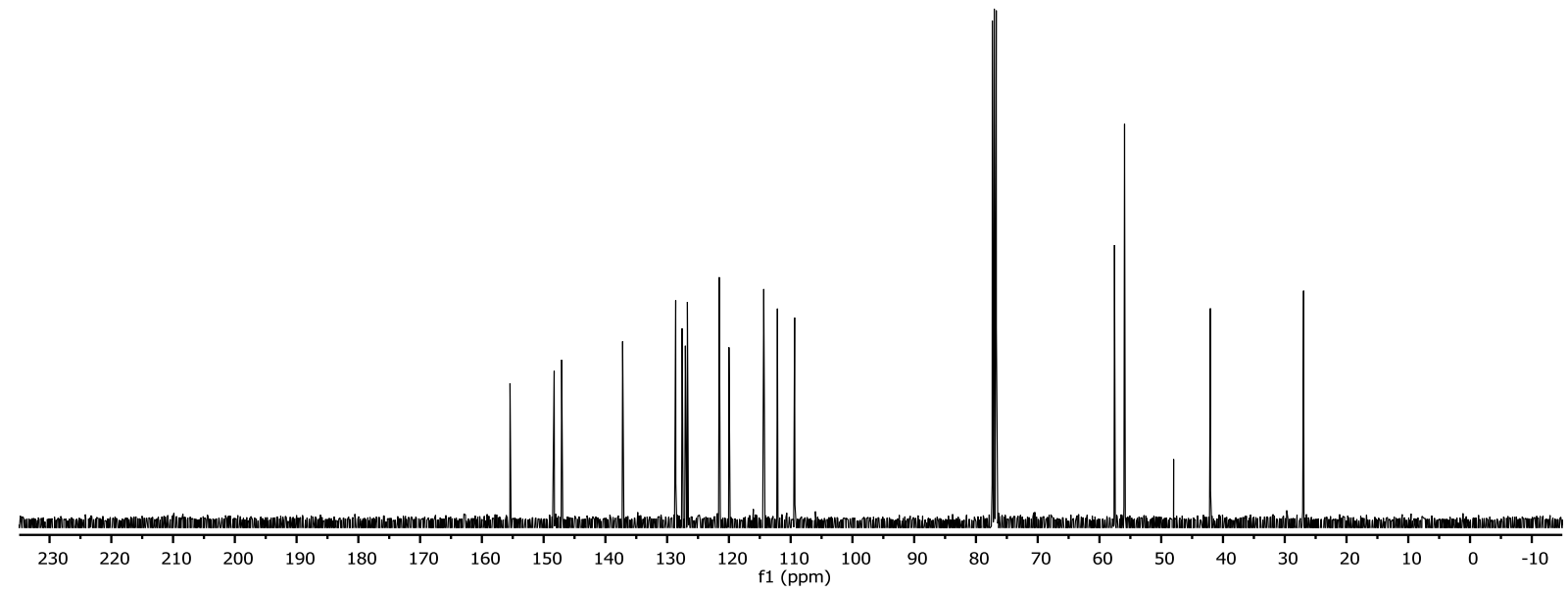

$16 a$

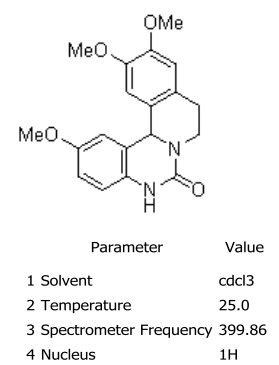

贷
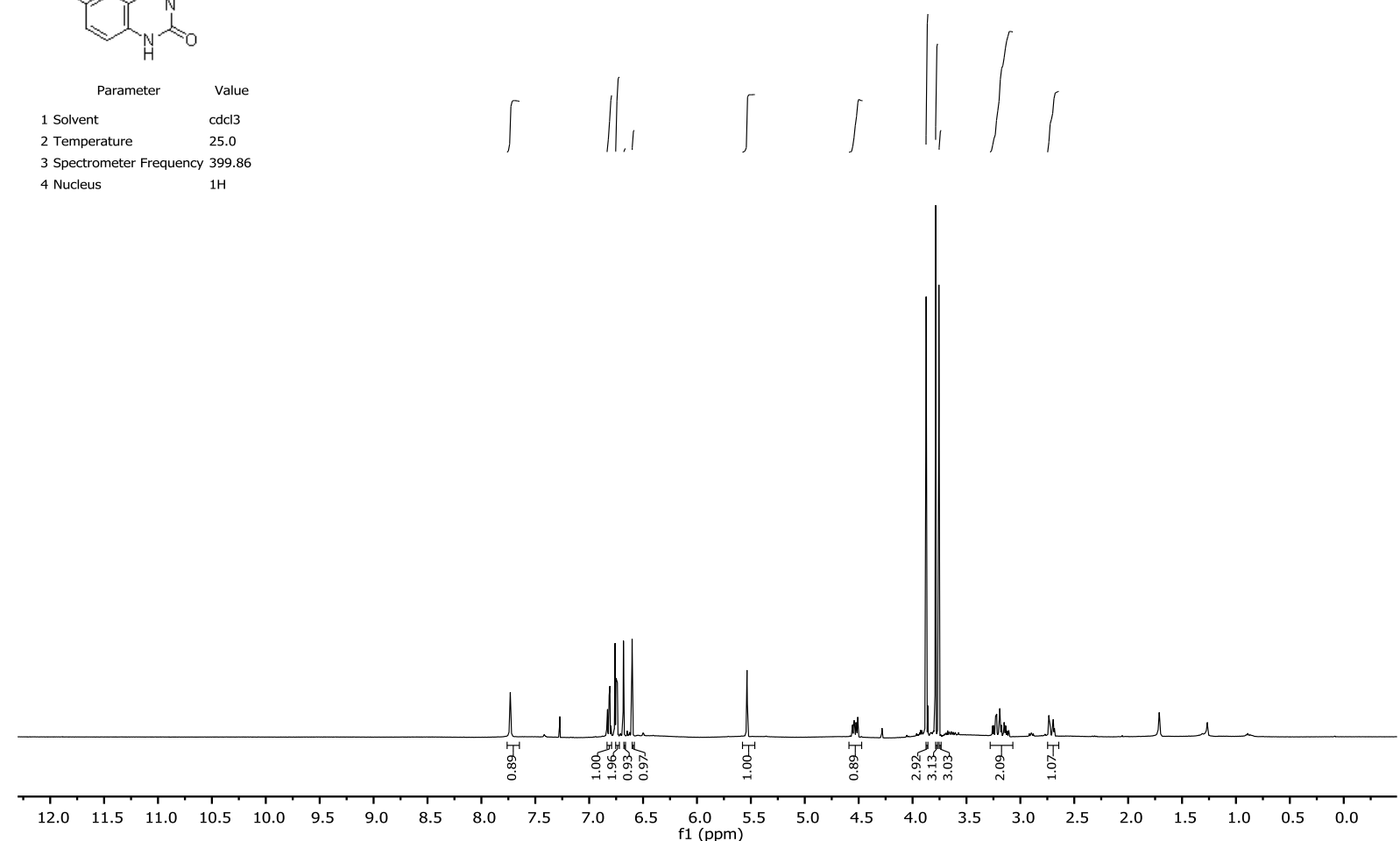


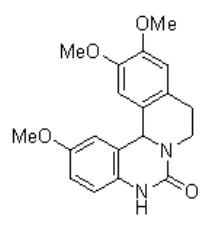

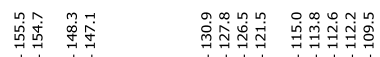

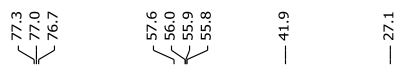

\begin{tabular}{ll}
\multicolumn{1}{c}{ Parameter } & \multicolumn{1}{c}{ Value } \\
1 Solvent & cdc13 \\
2 Temperature & 25.0 \\
3 Spectrometer Frequency & 100.55 \\
4 Nucleus & $13 \mathrm{C}$
\end{tabular}

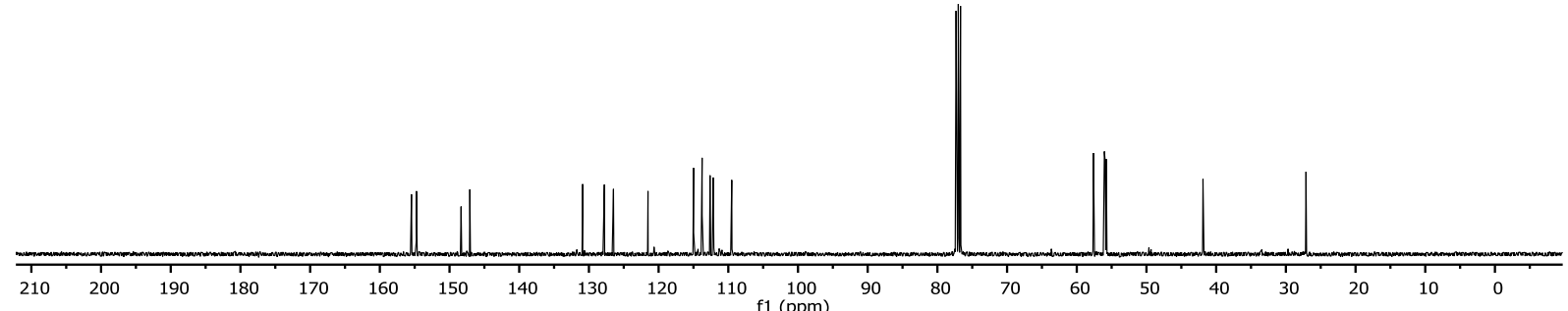

$16 b$ 

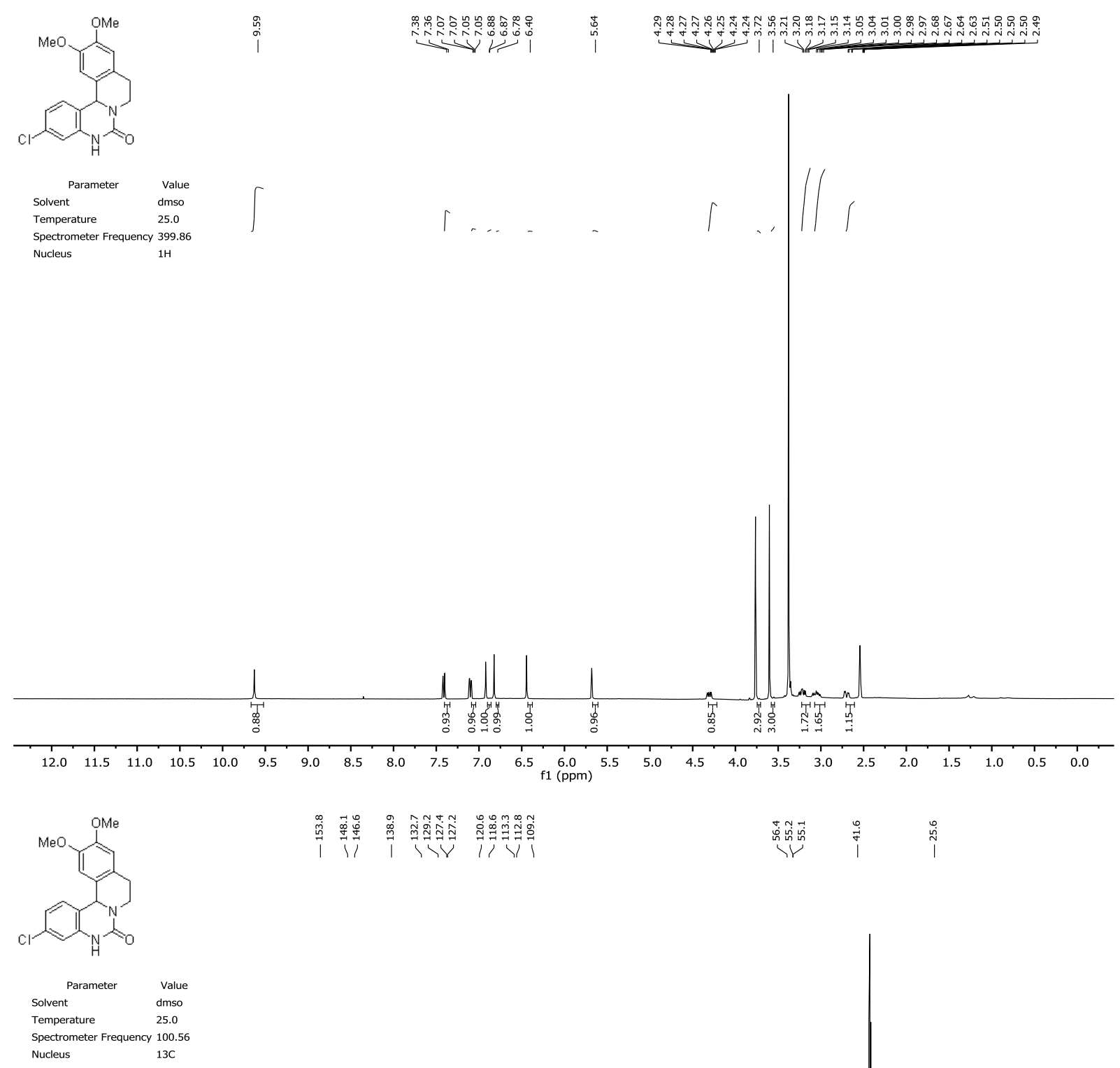

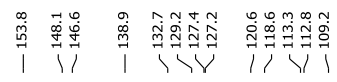

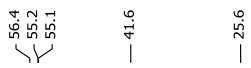

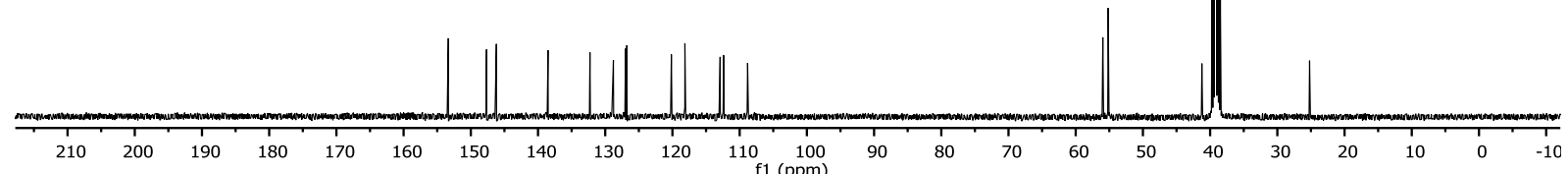



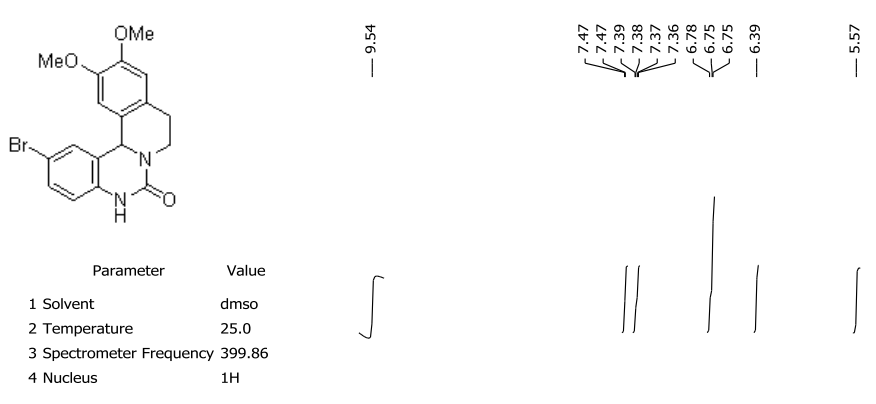

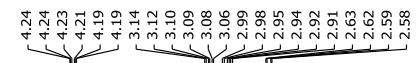
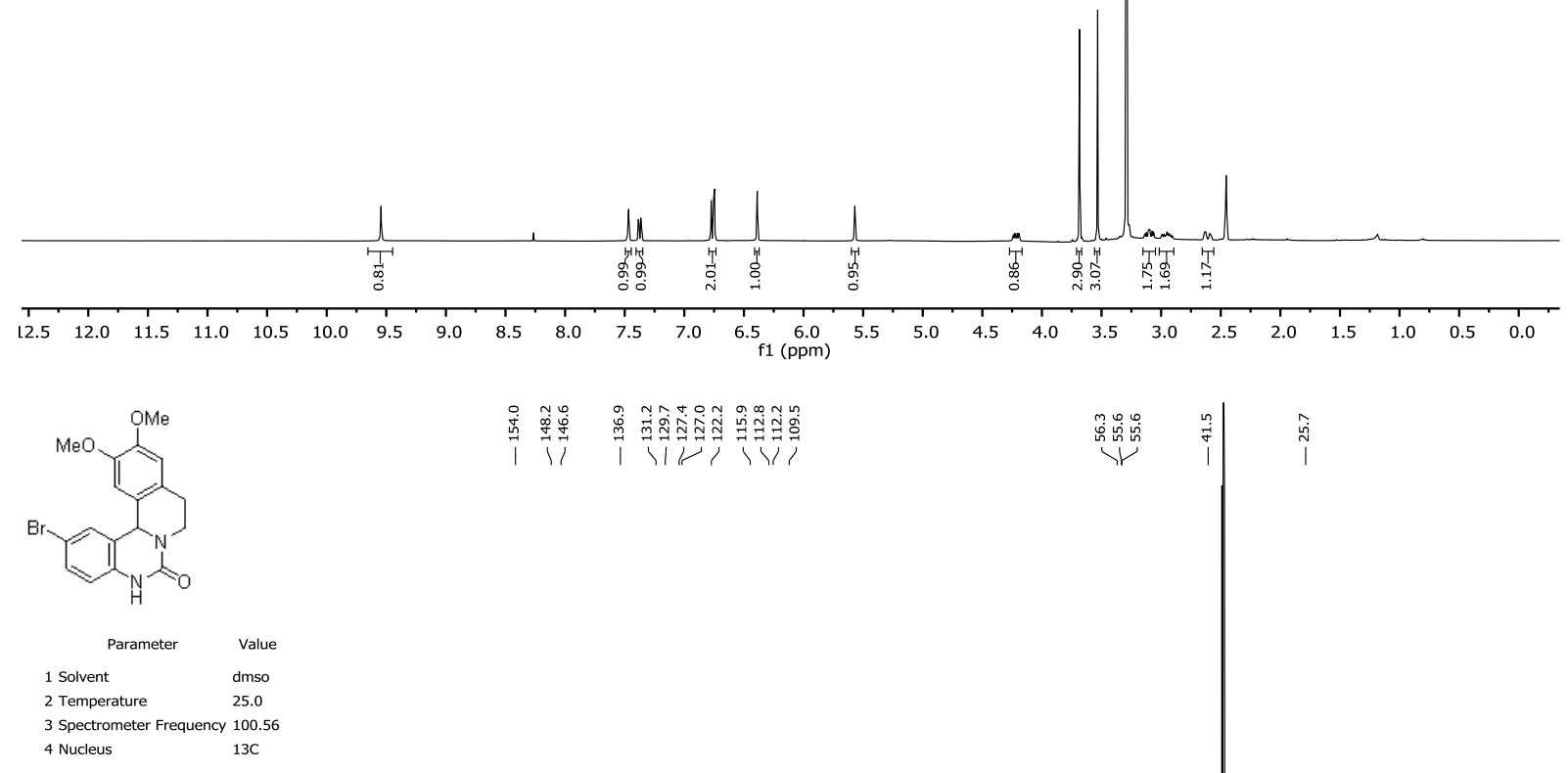

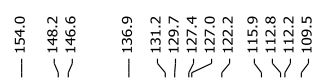 \\ मा}

16d 

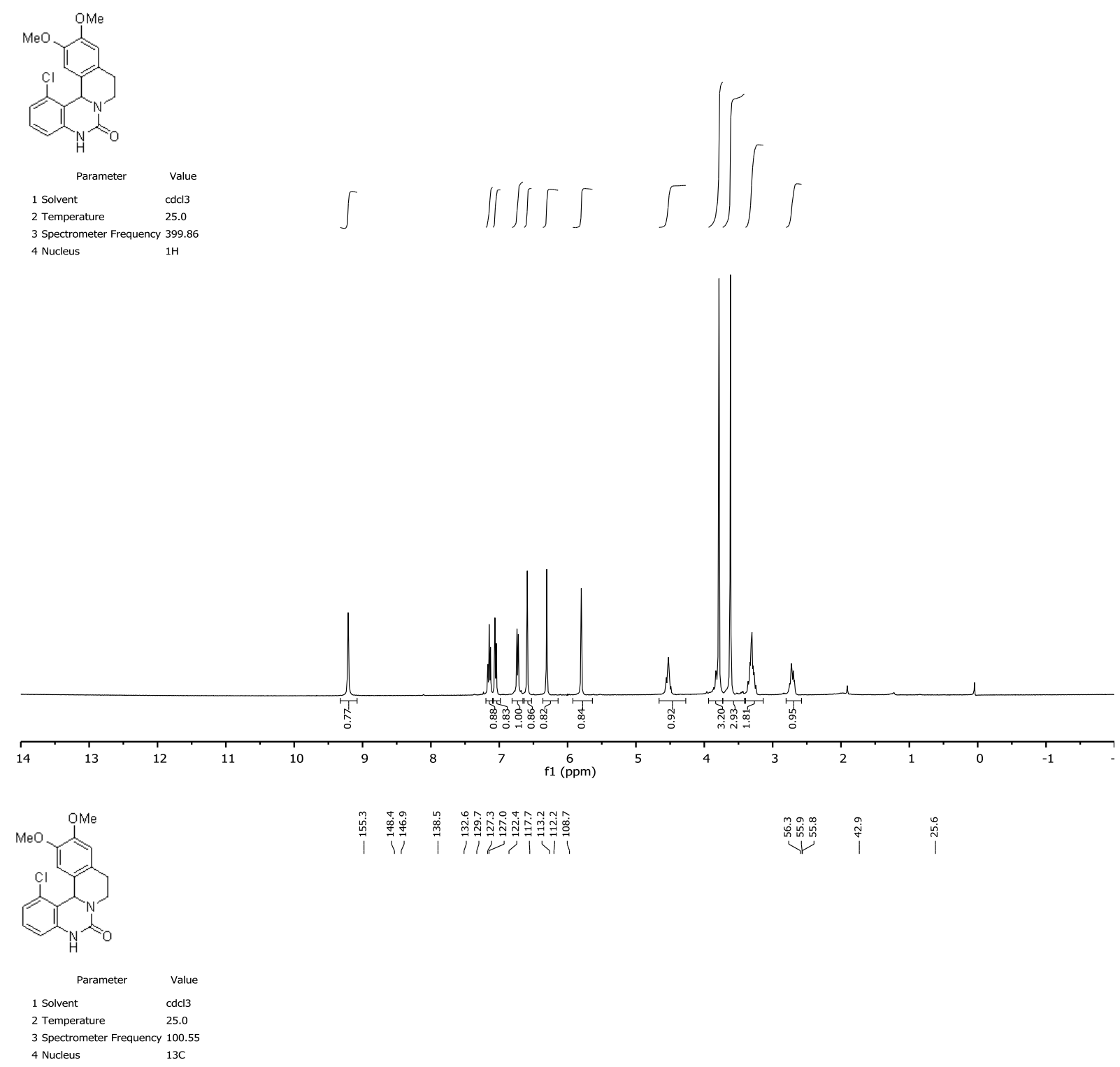

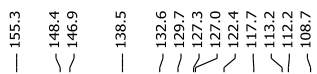

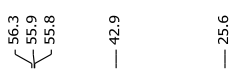

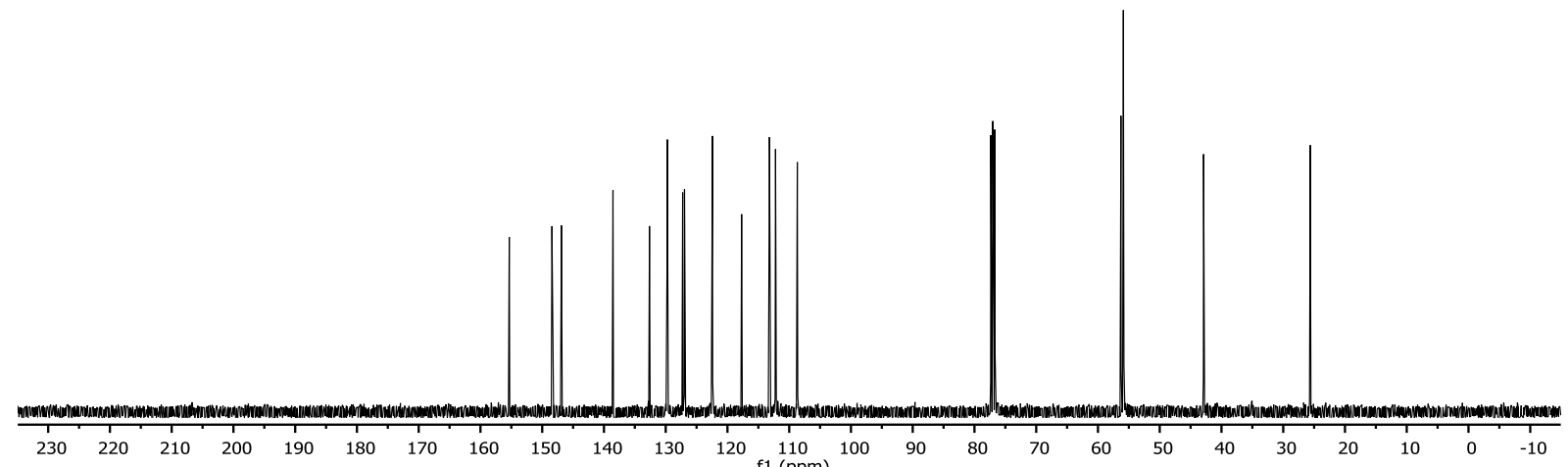

$16 e$ 

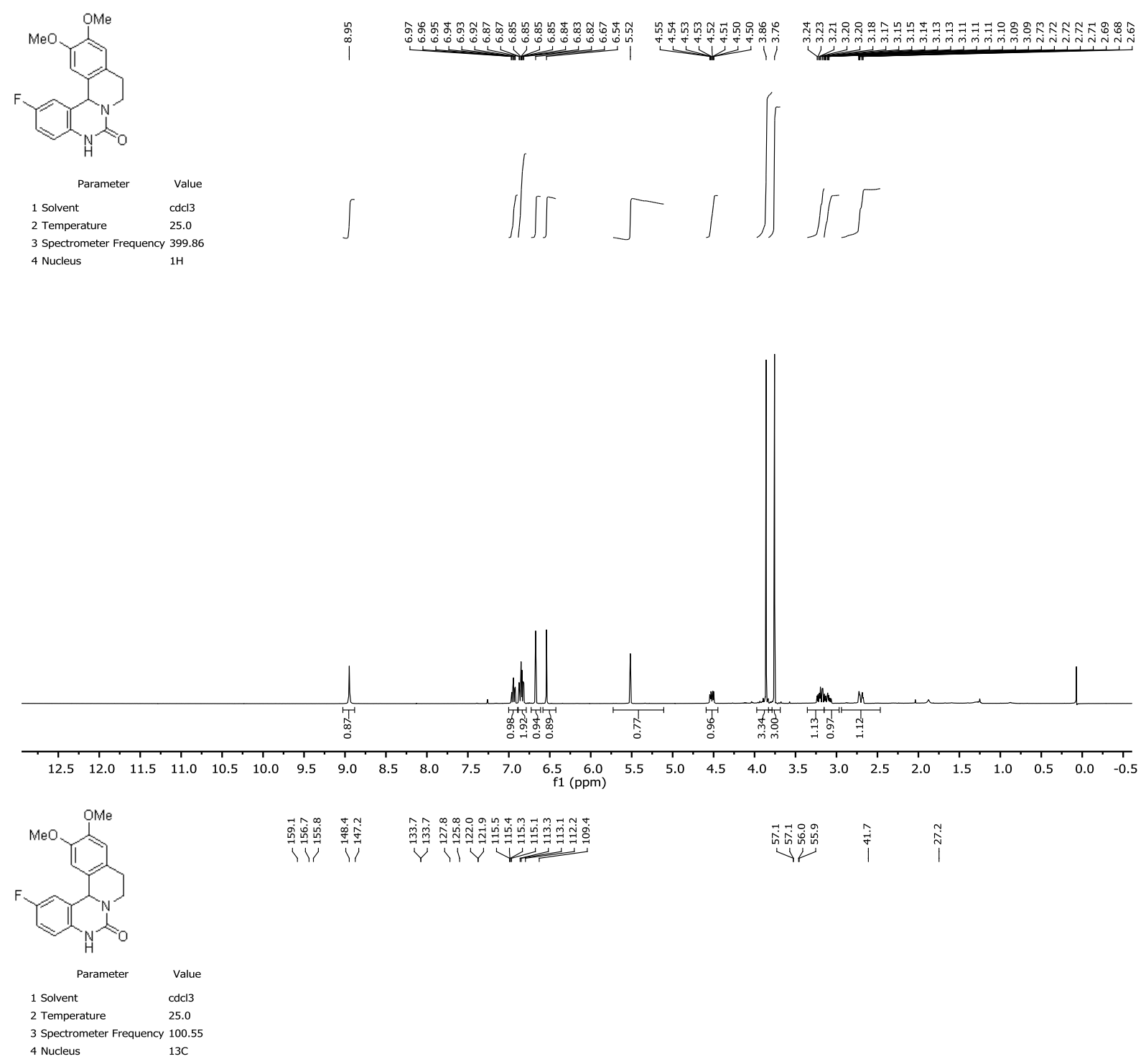

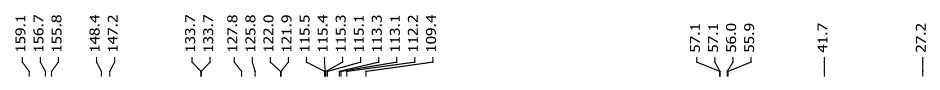

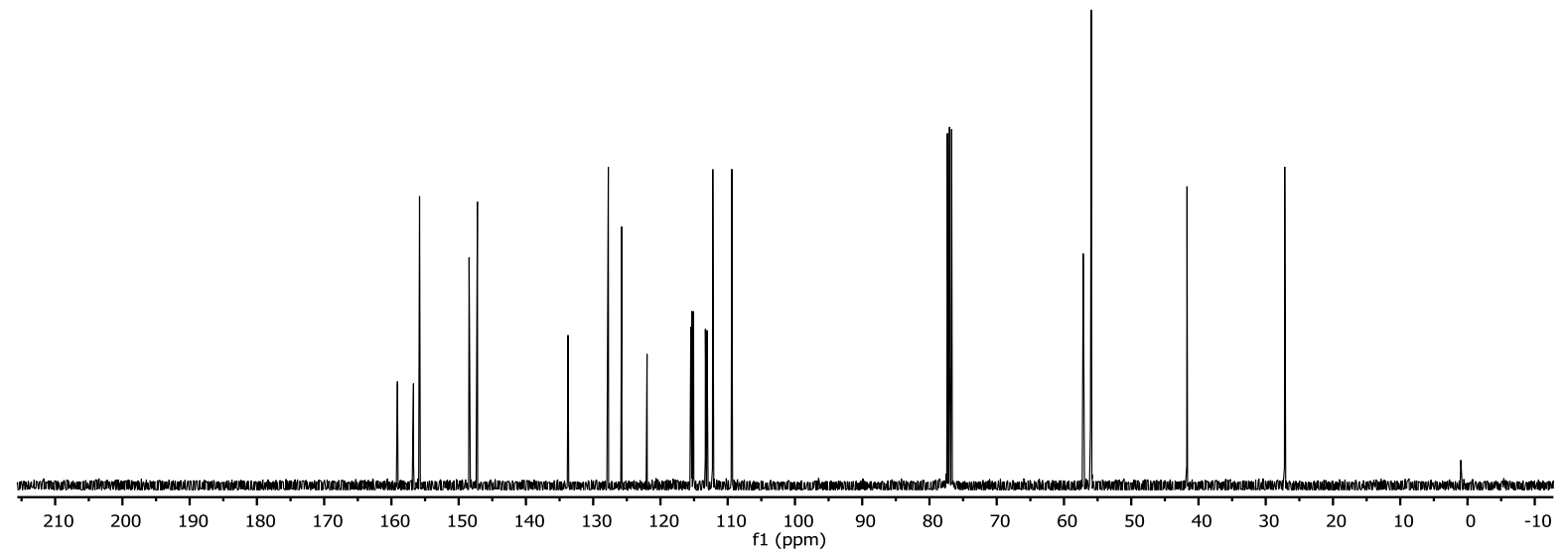




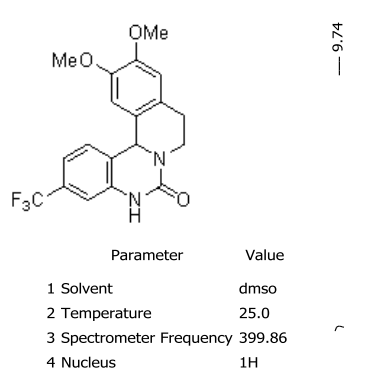

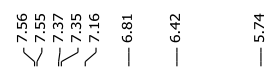
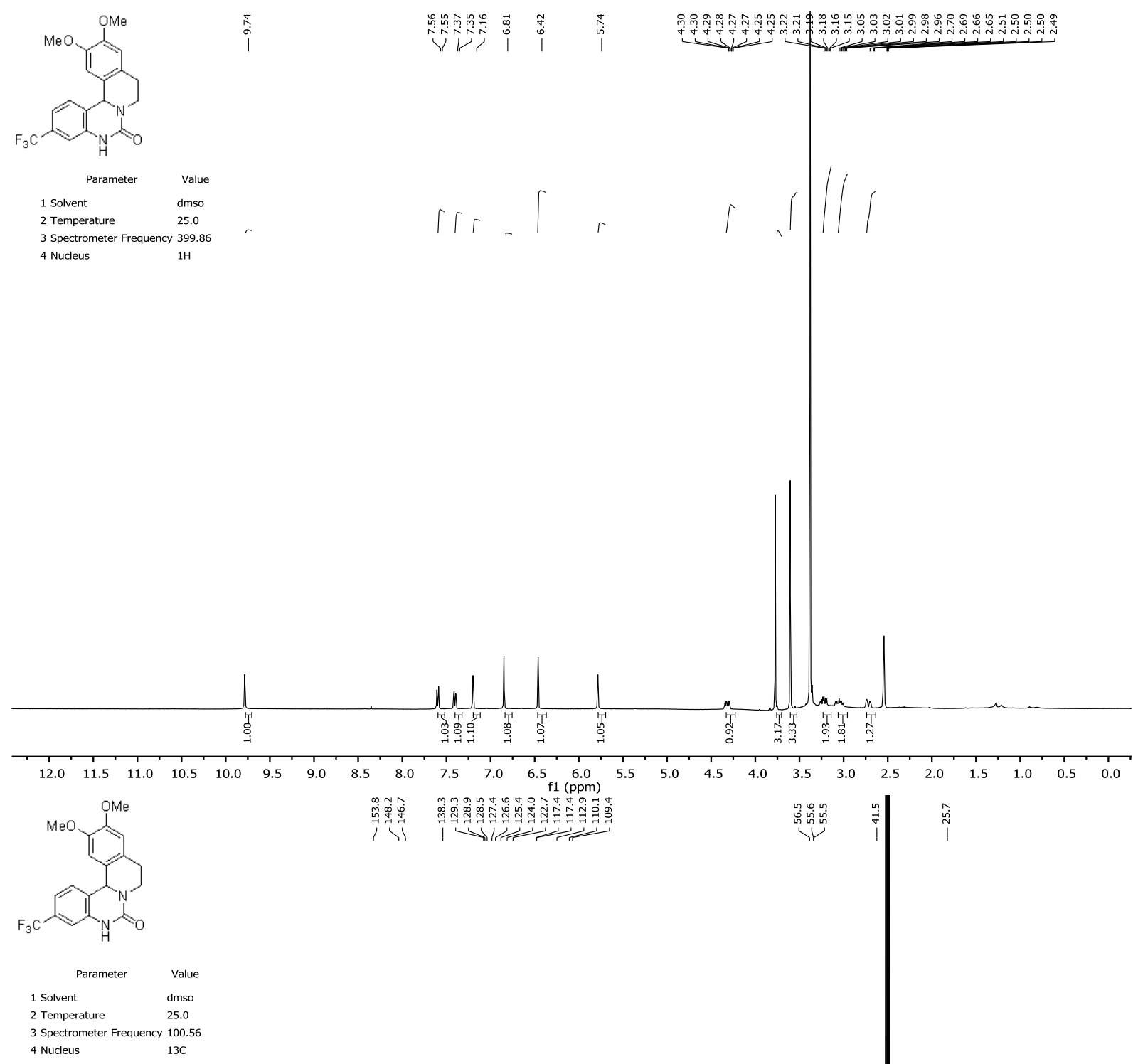

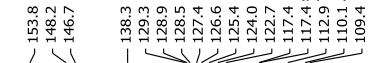
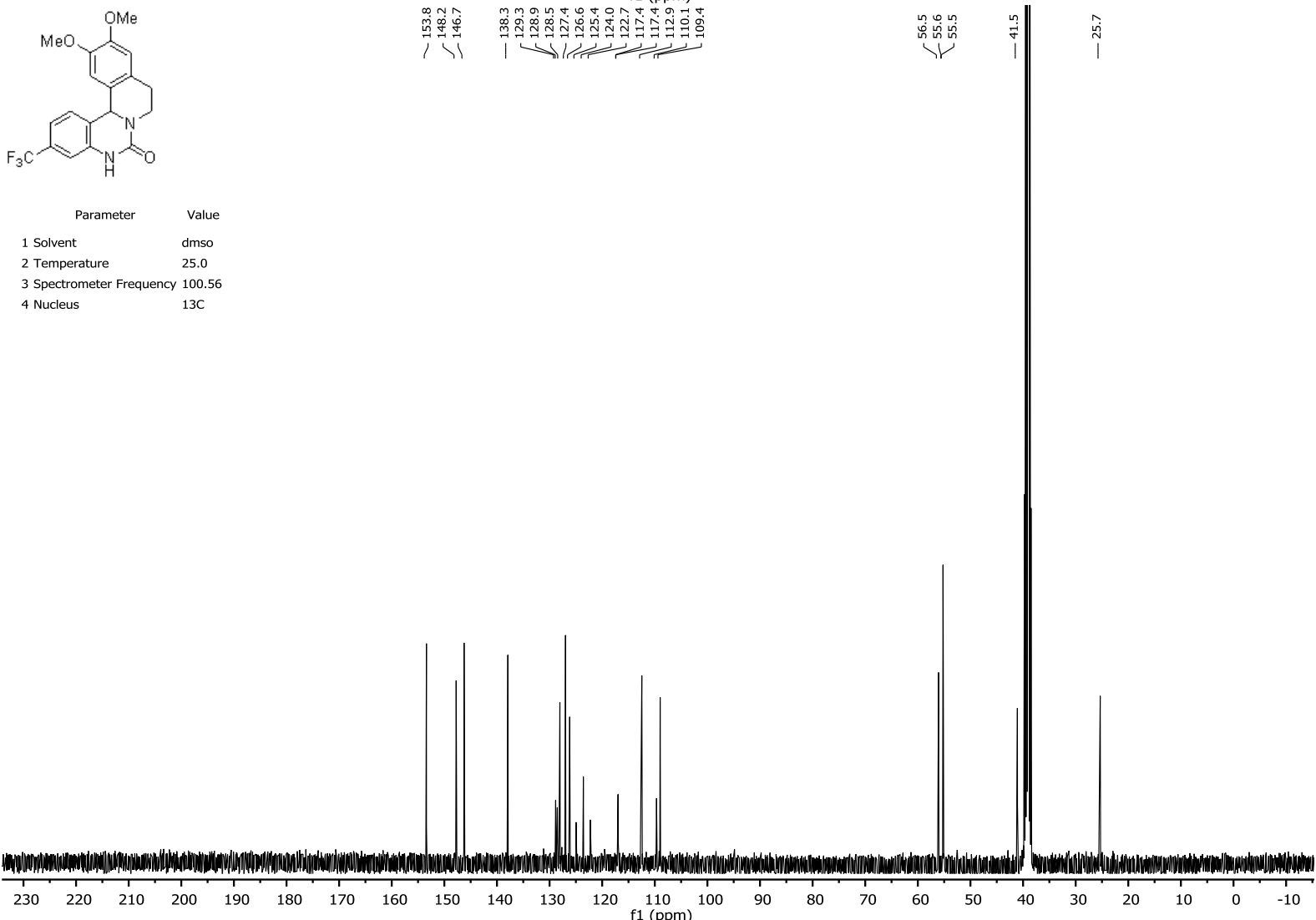

$16 \mathrm{~g}$ 


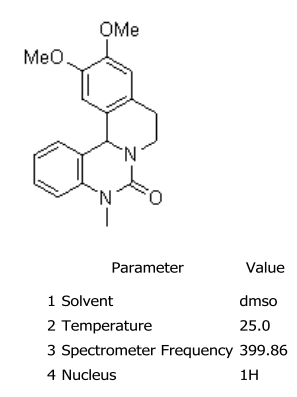

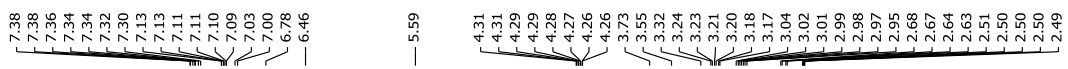

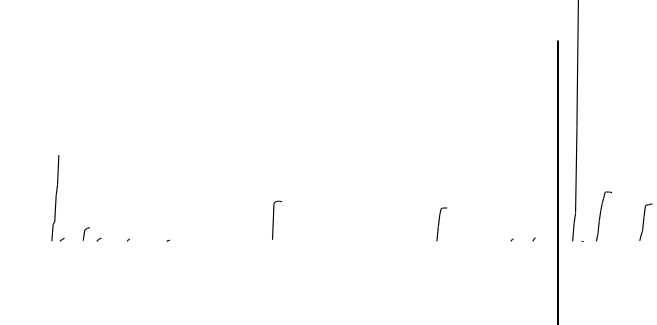

Nill

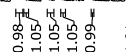

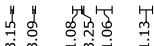

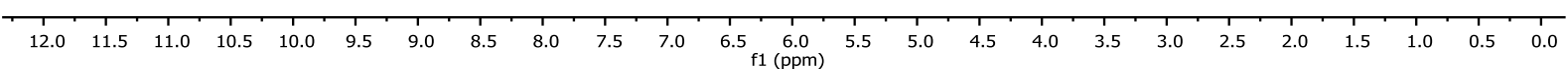

(1)

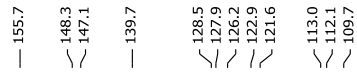

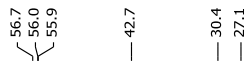
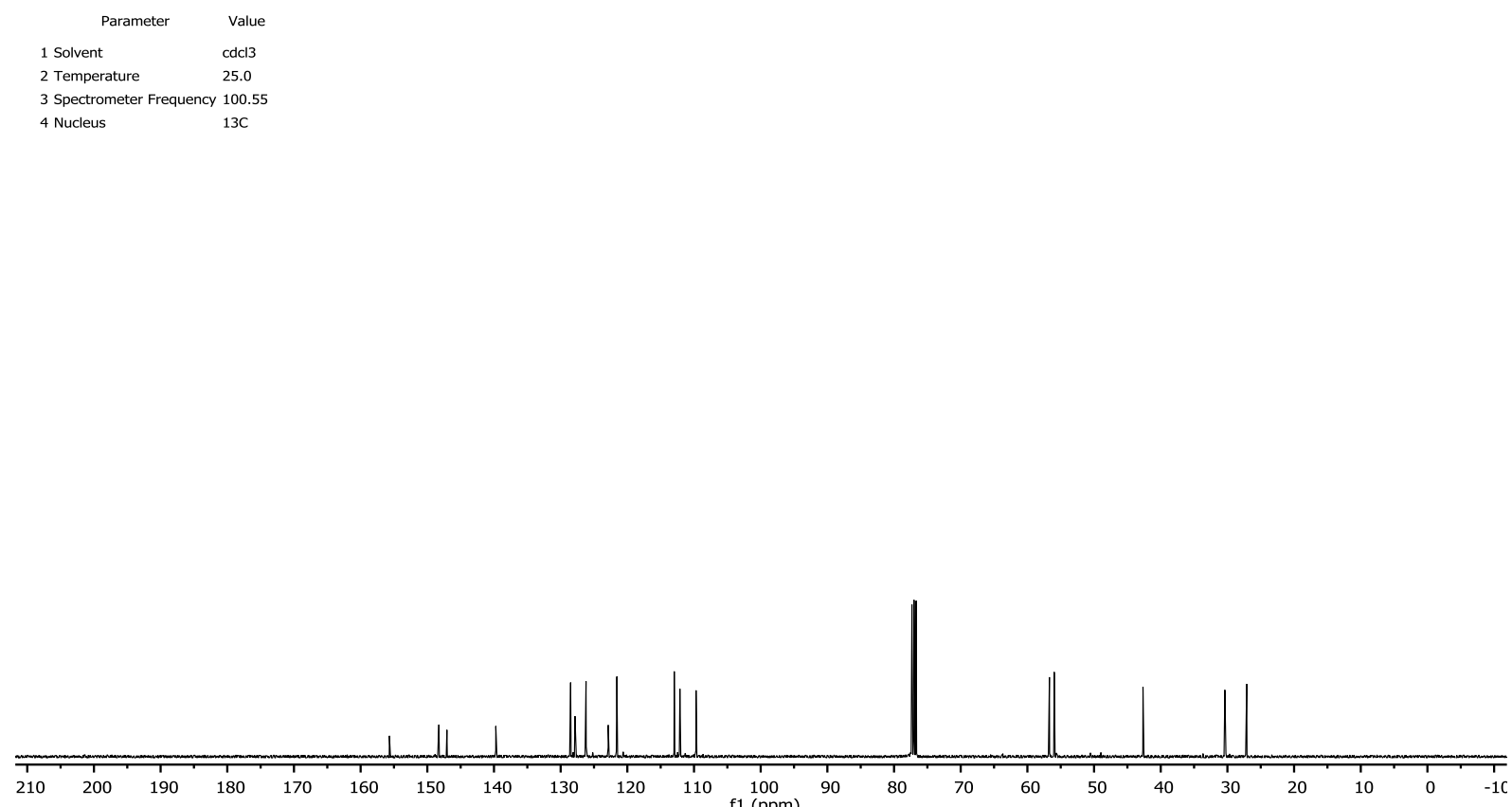

$16 h$ 


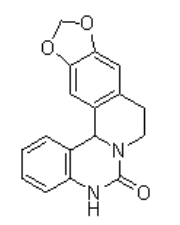

$\underbrace{7}$
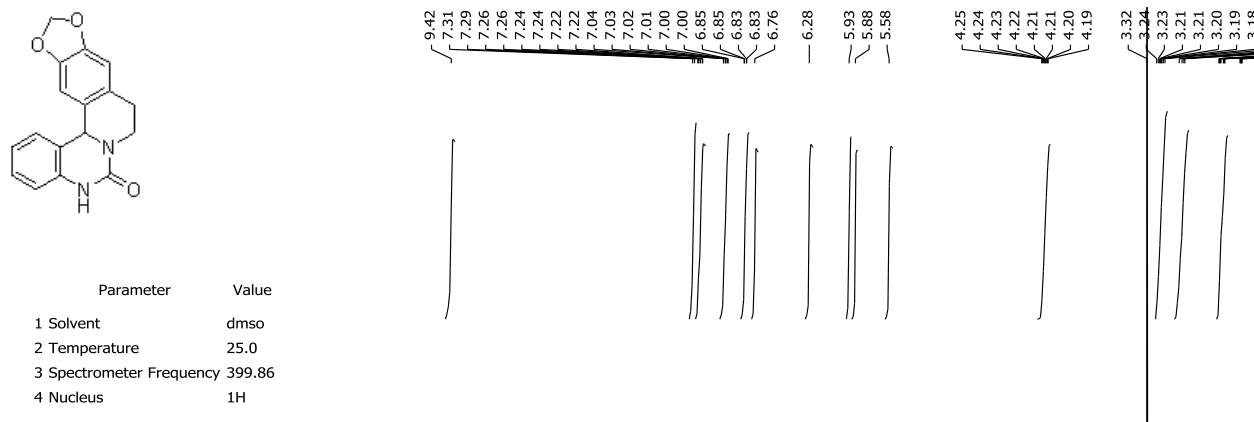

2 Temperature
3 Spectrometer Frequency 399.86

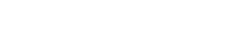




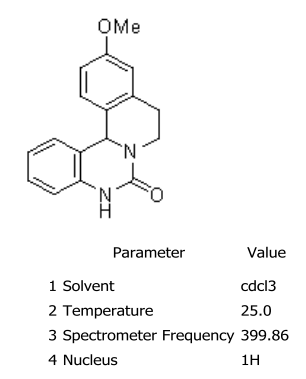

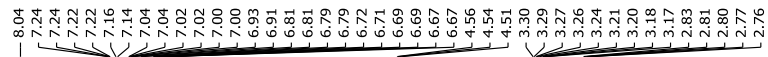
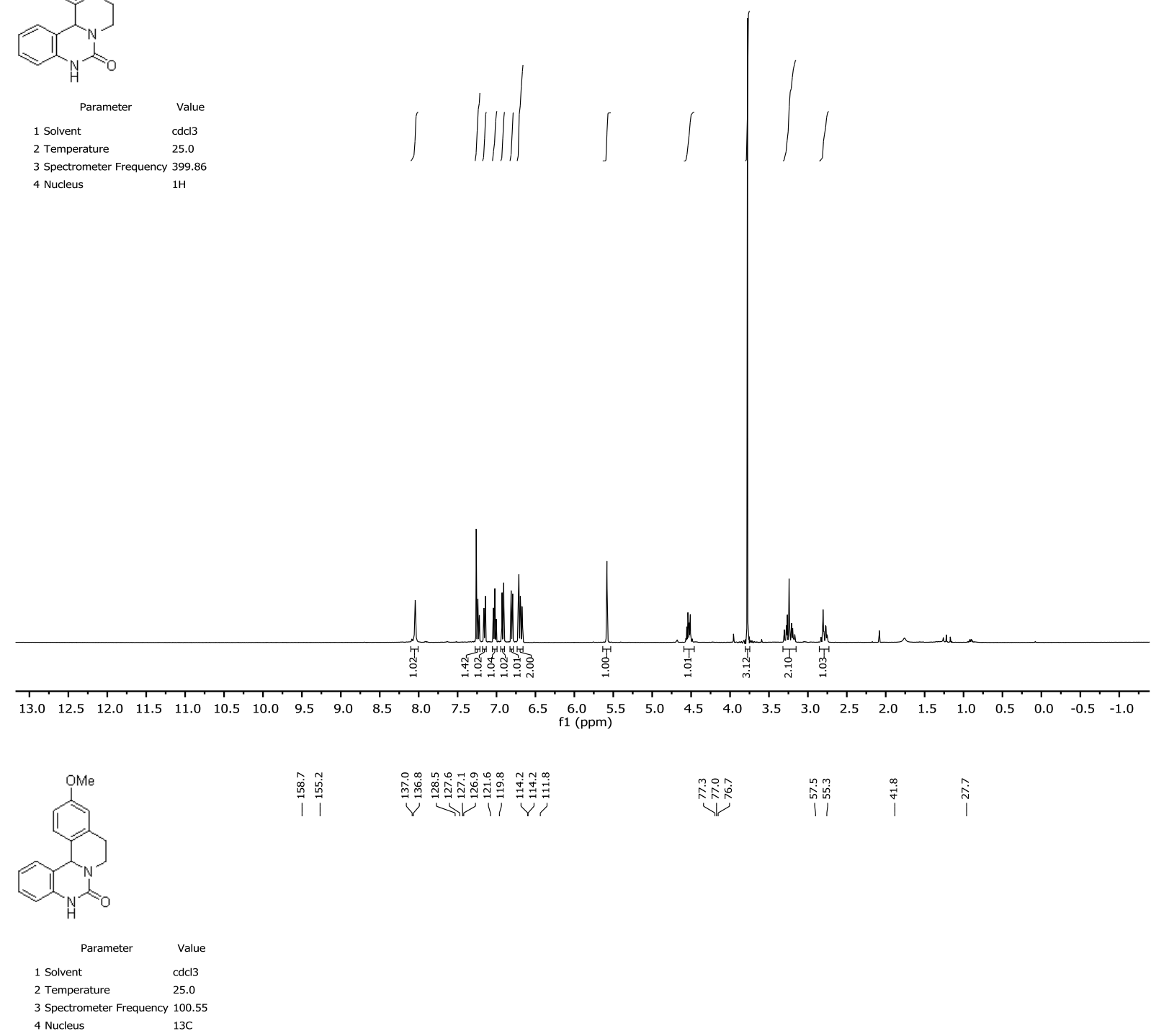

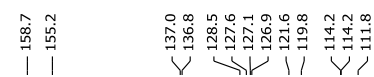

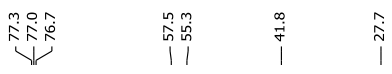

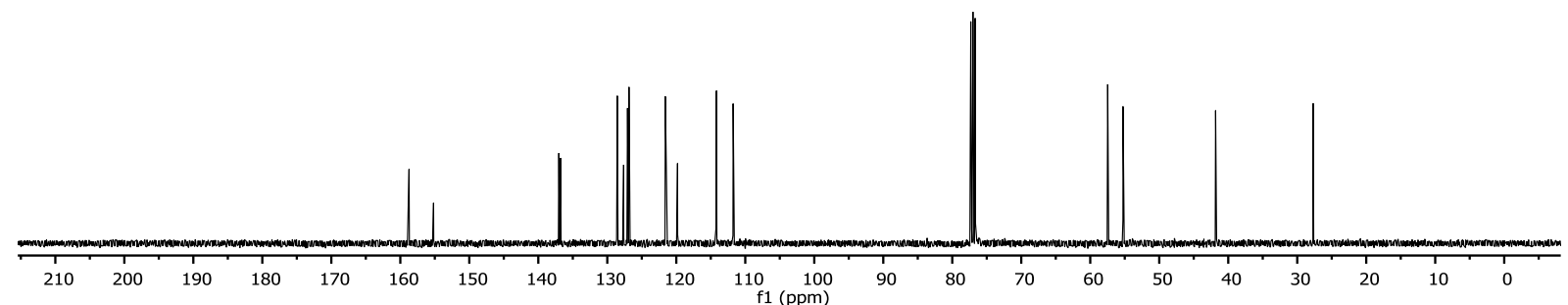



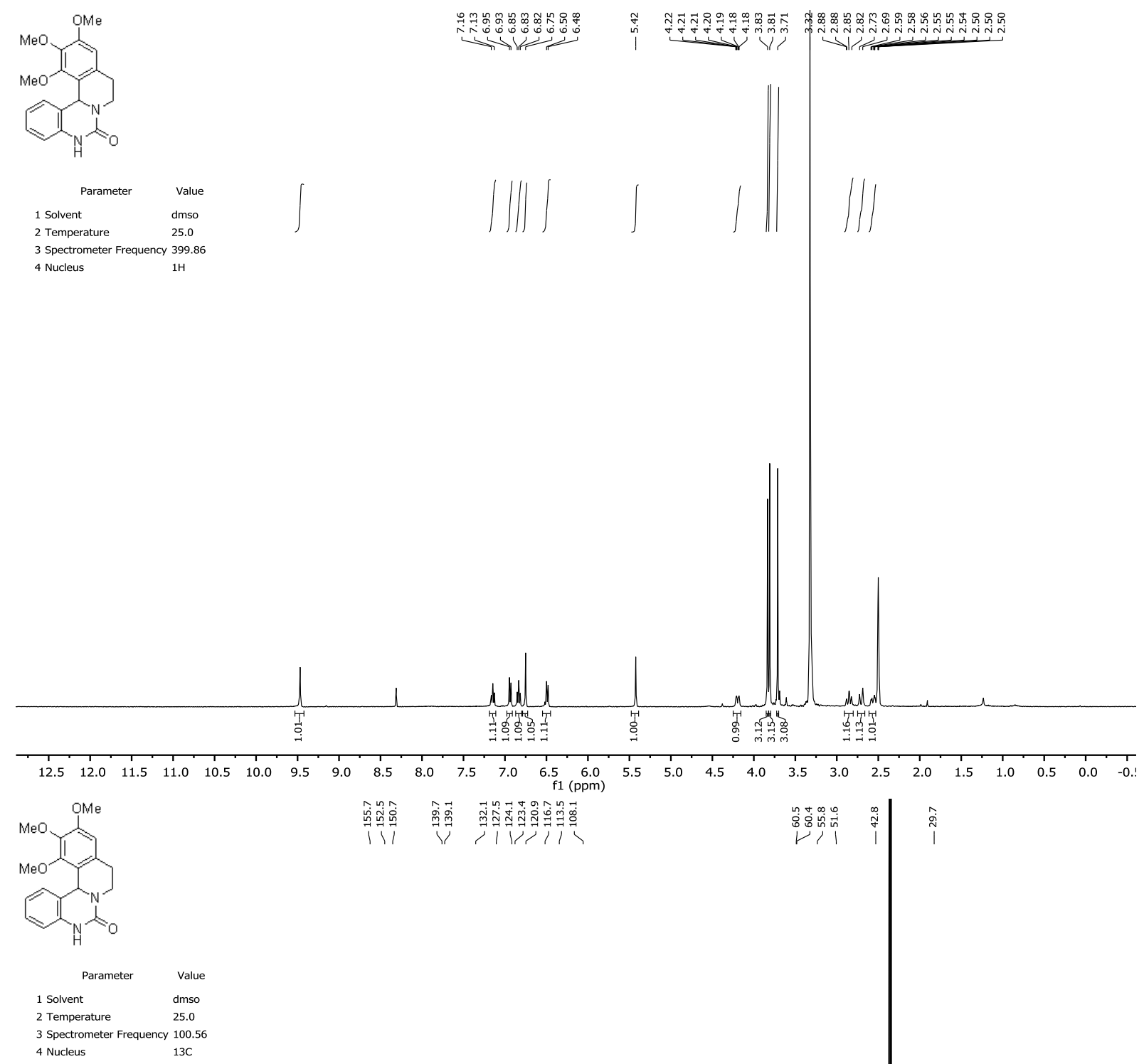

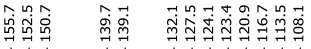

111 Y । |l|।

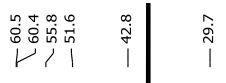

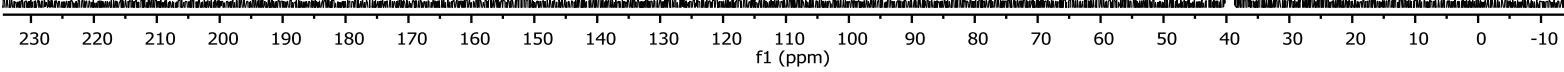




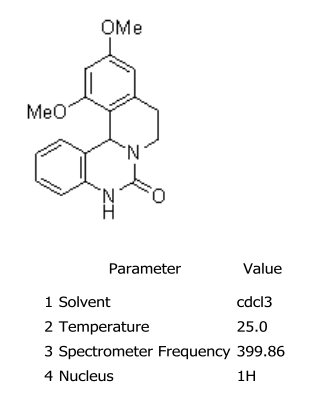

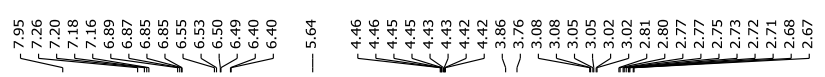
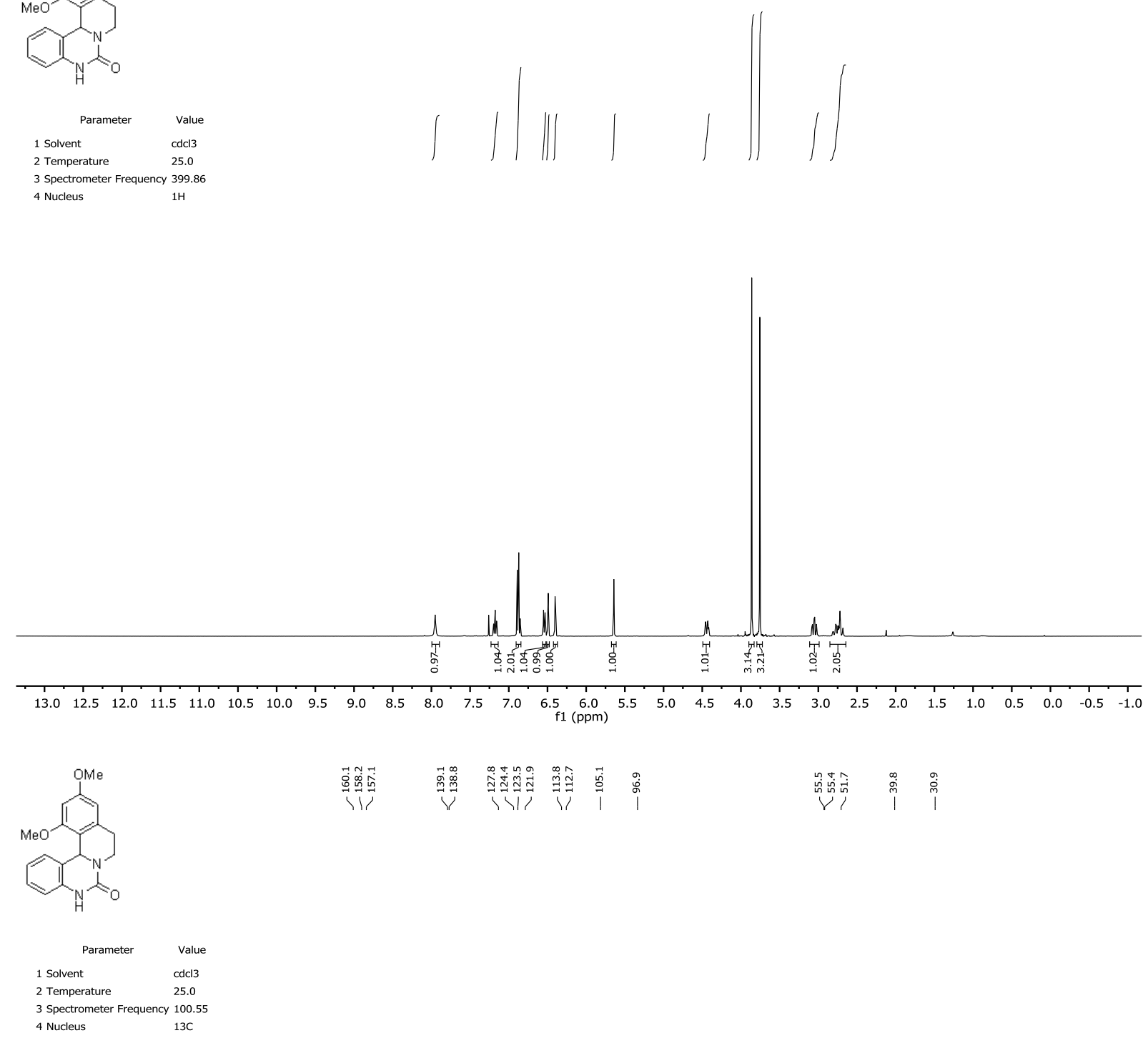

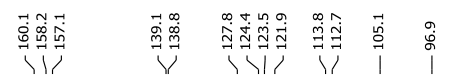

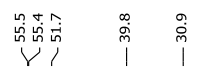

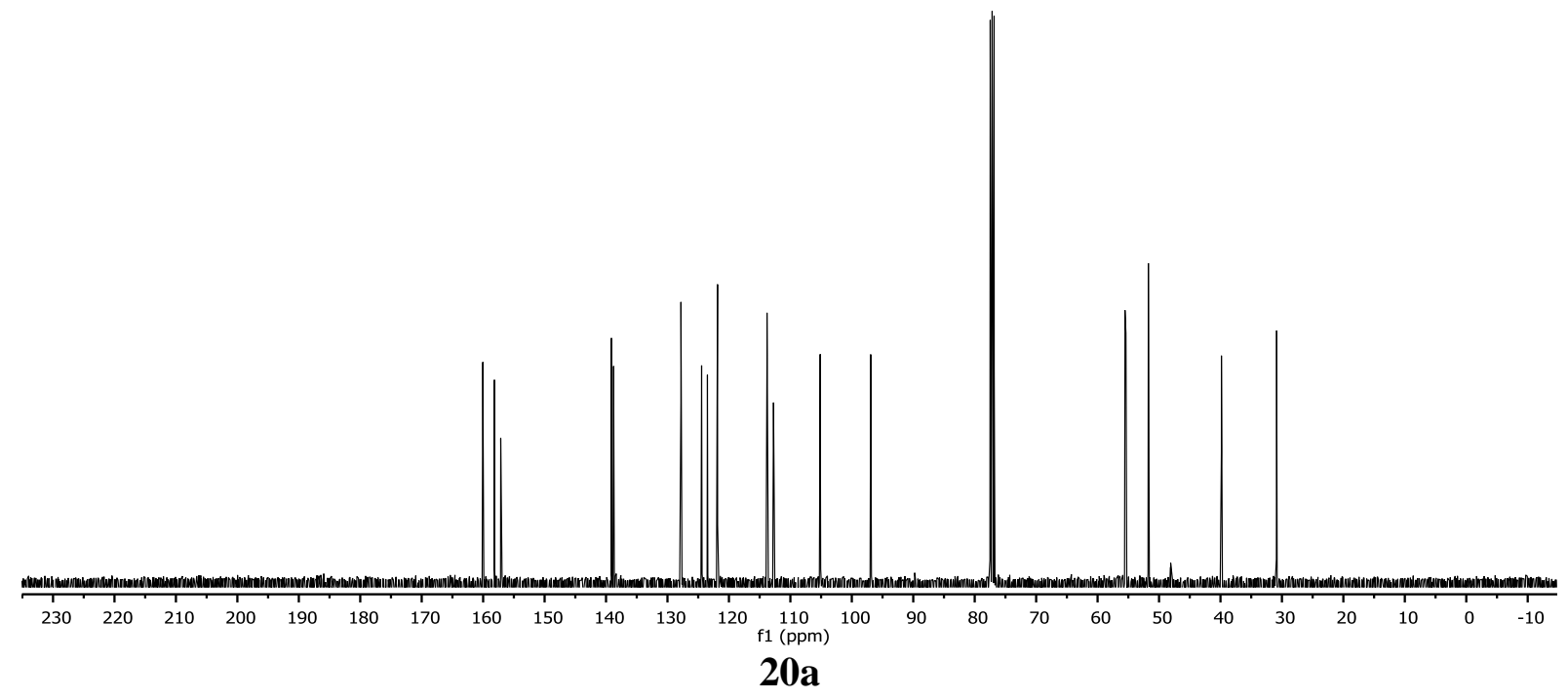




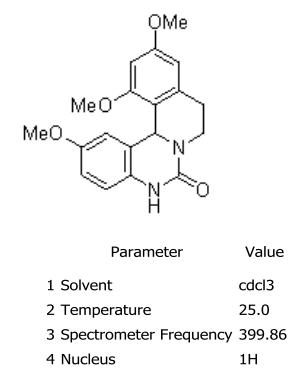

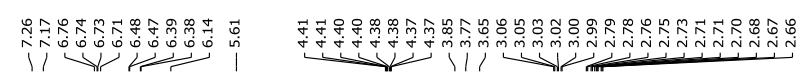
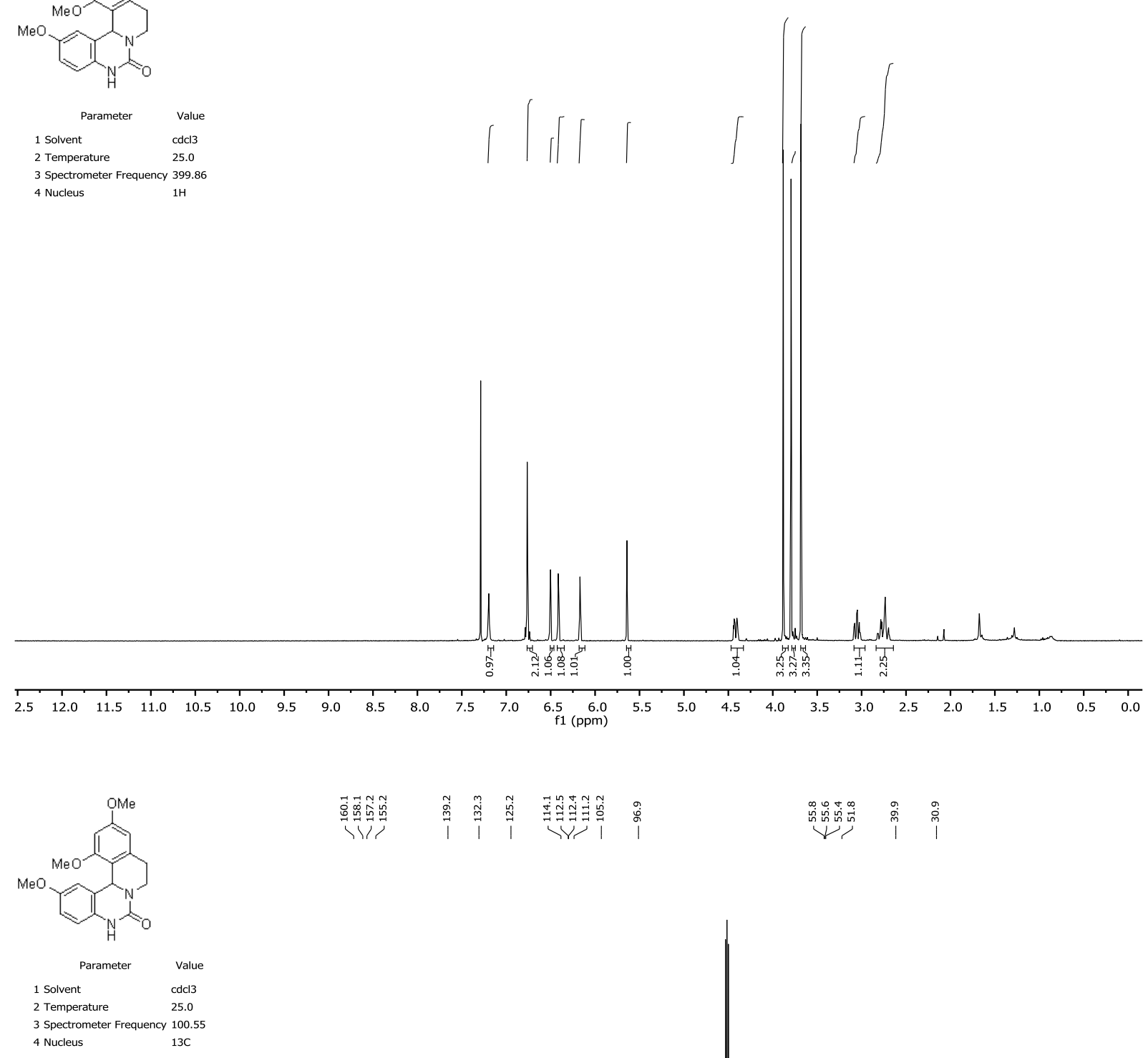

-

$$
\text { . }
$$



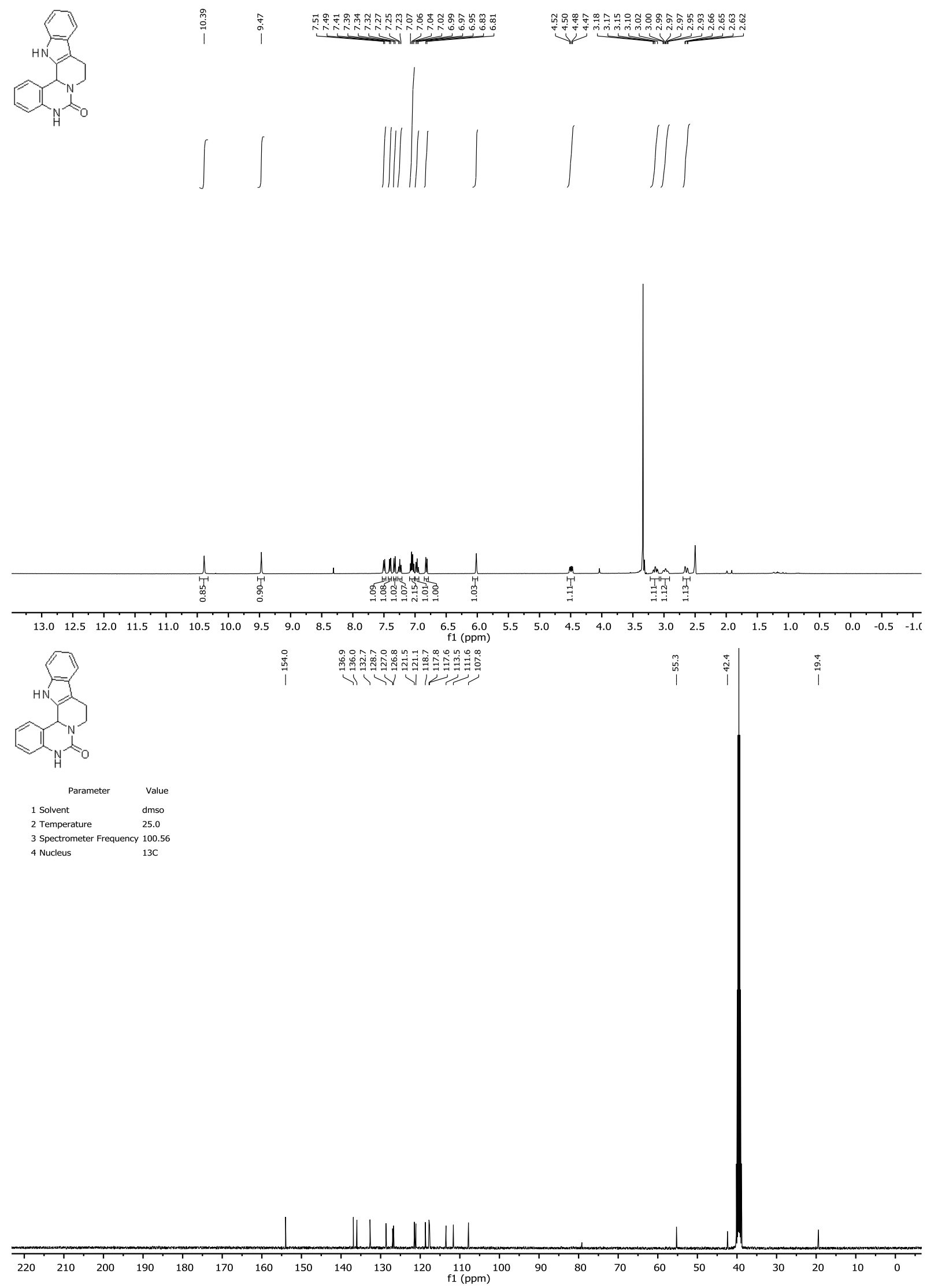

21a 

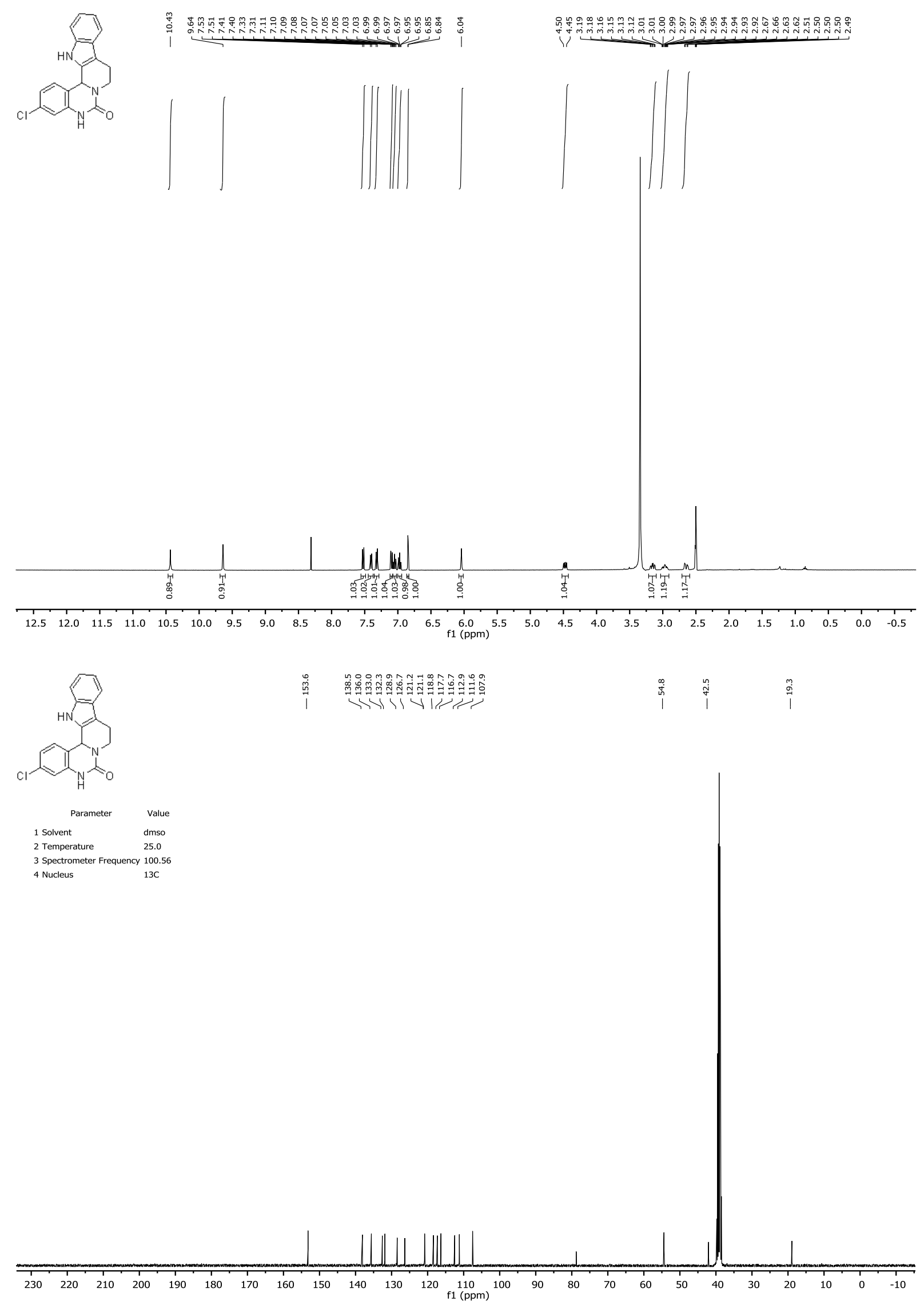

21b 

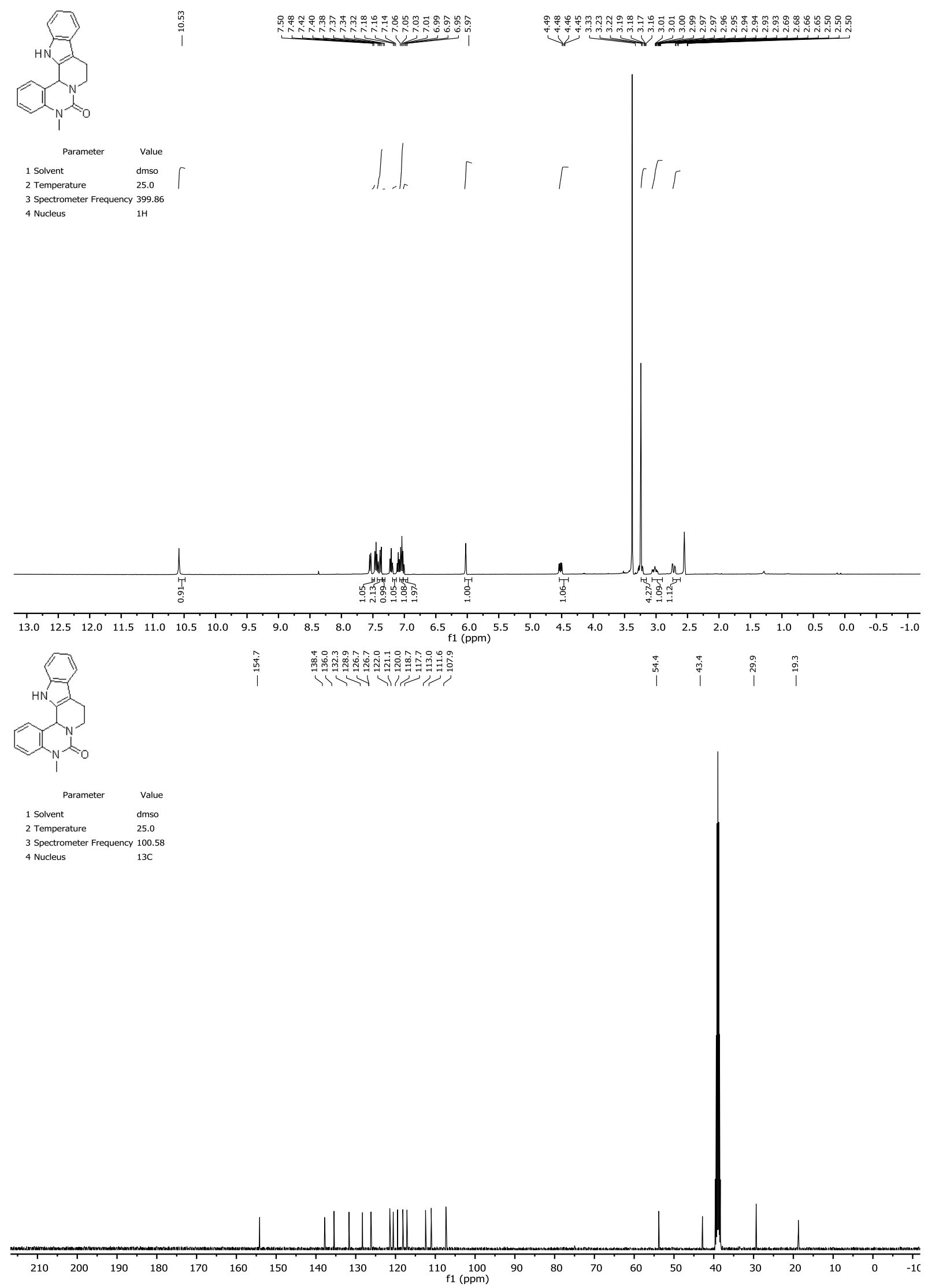

21c 


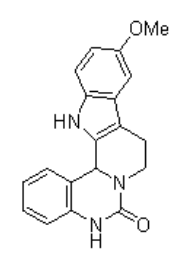

蛋

Parameter

1 Solvent

2 Temperature

4 Nucleus

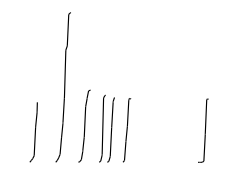

${ }_{11}^{399.8}$

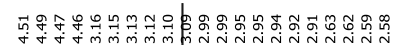
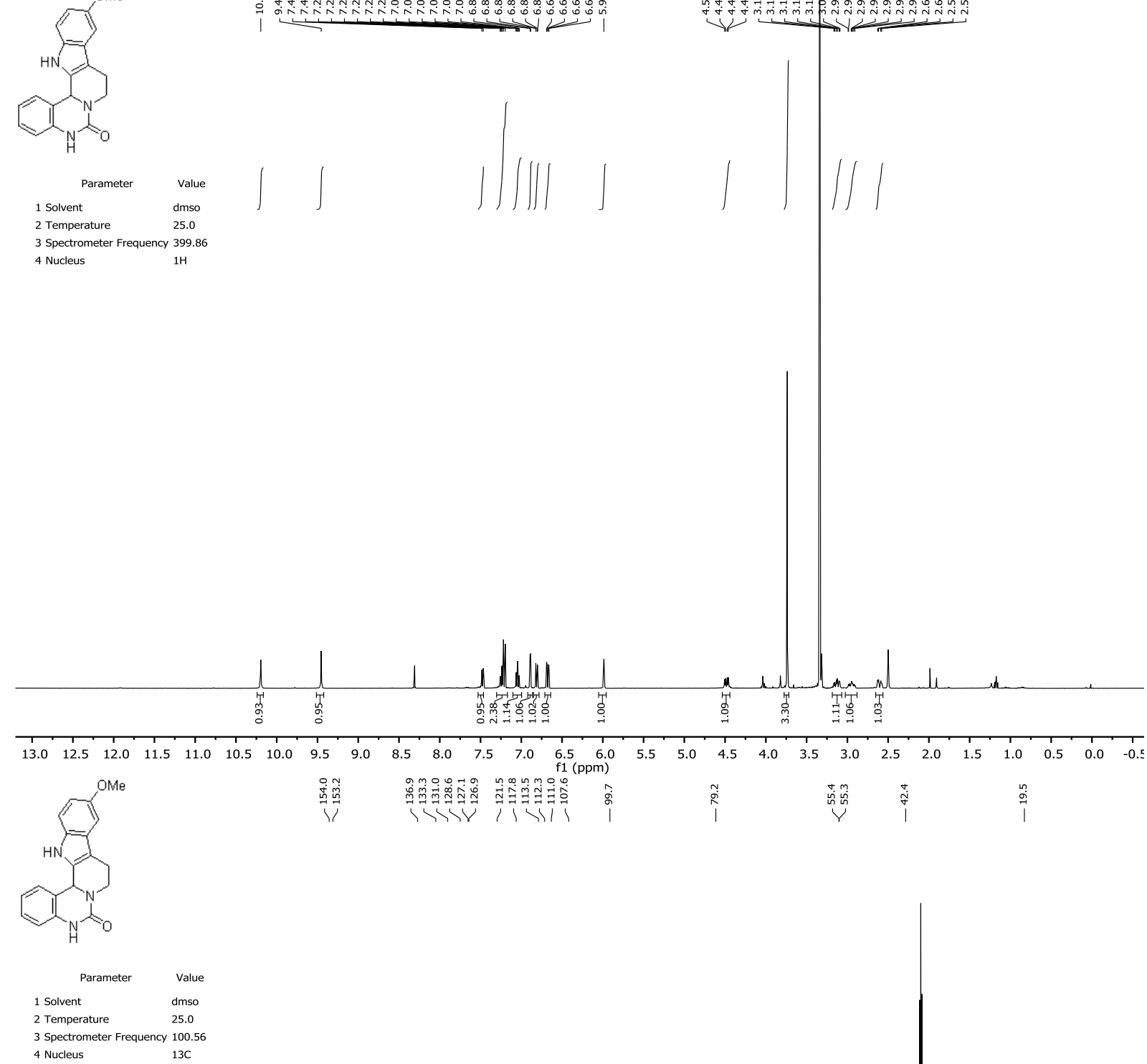

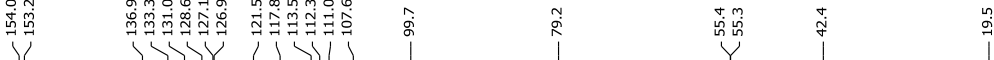

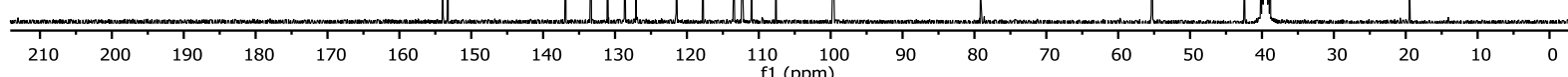

22 

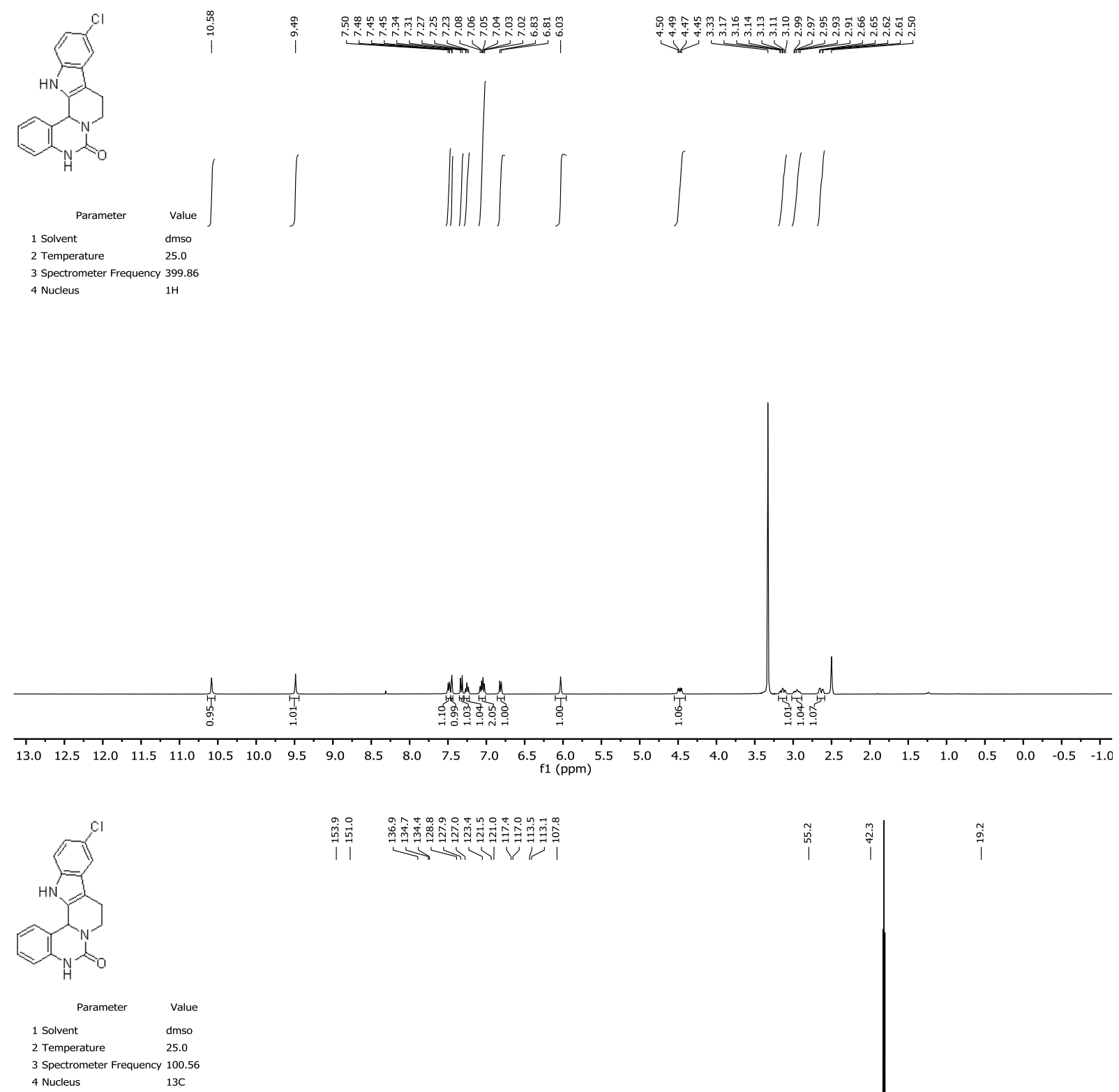

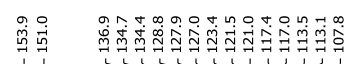

। ।

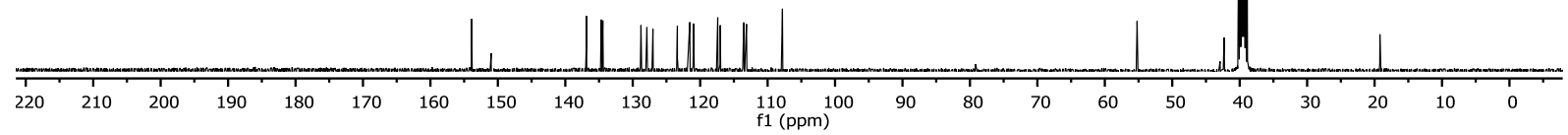



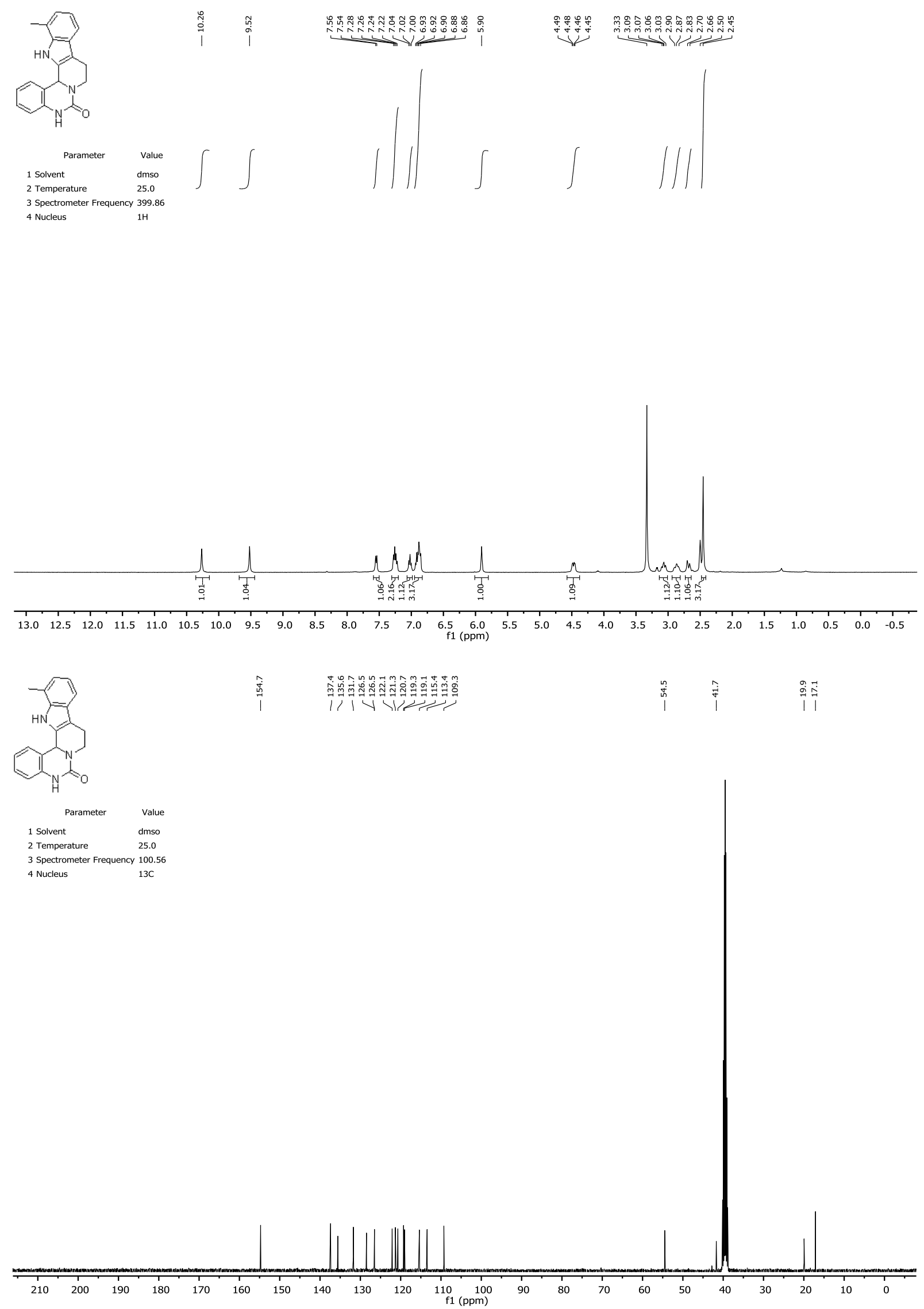


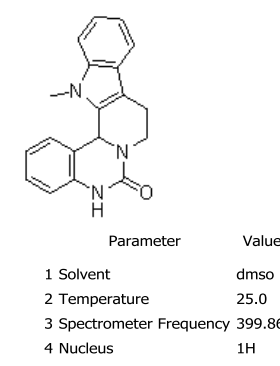

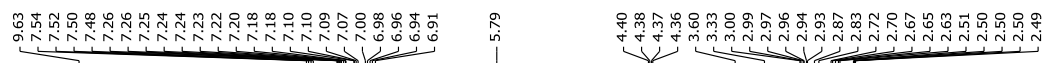
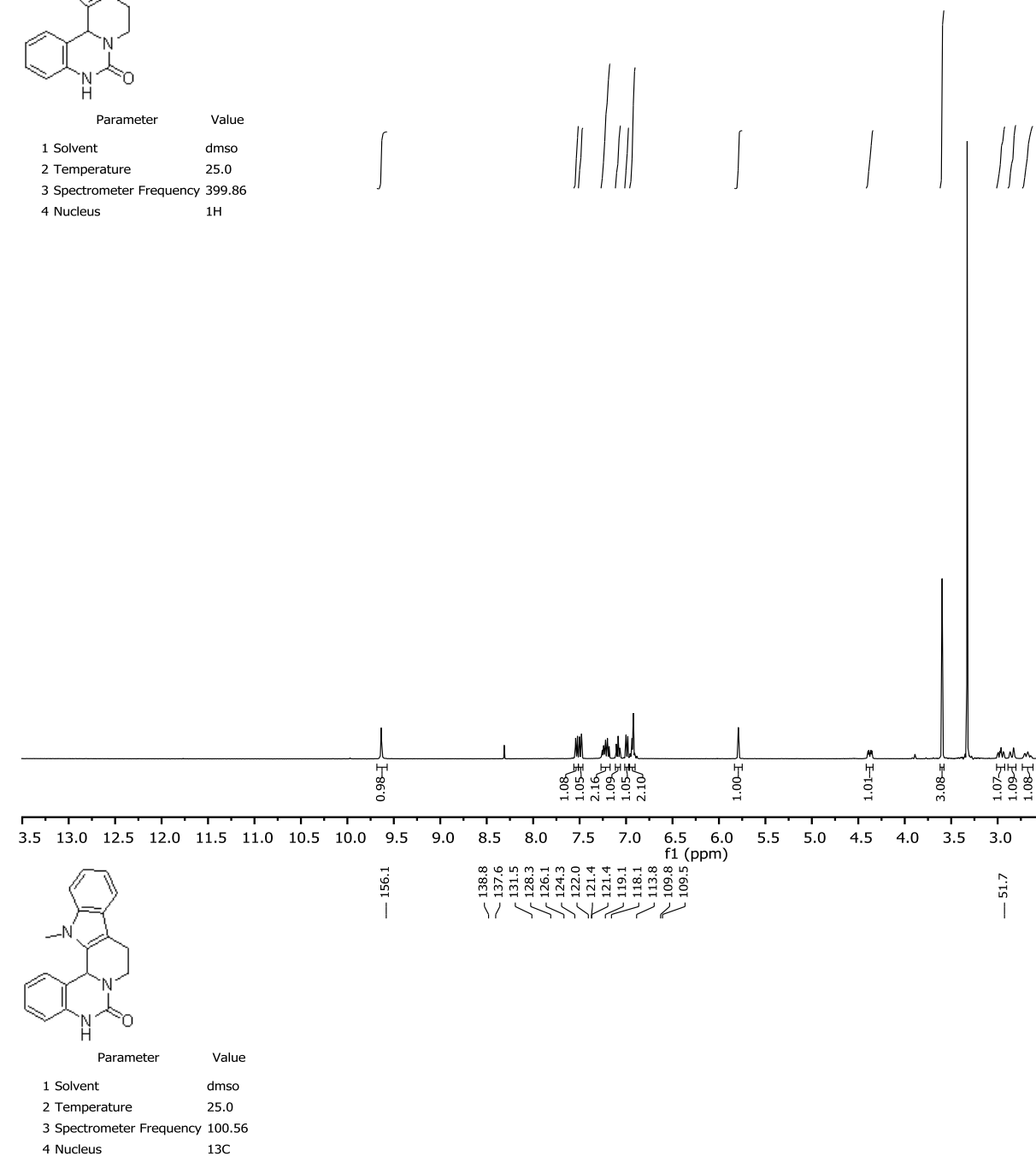

管等要

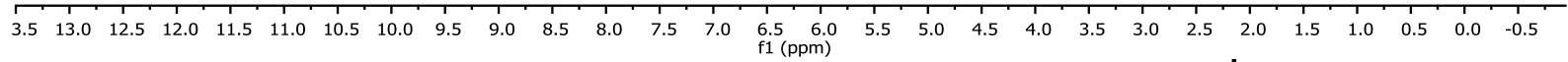

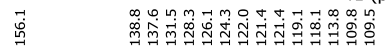

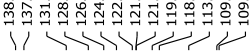

$13 \mathrm{C}$

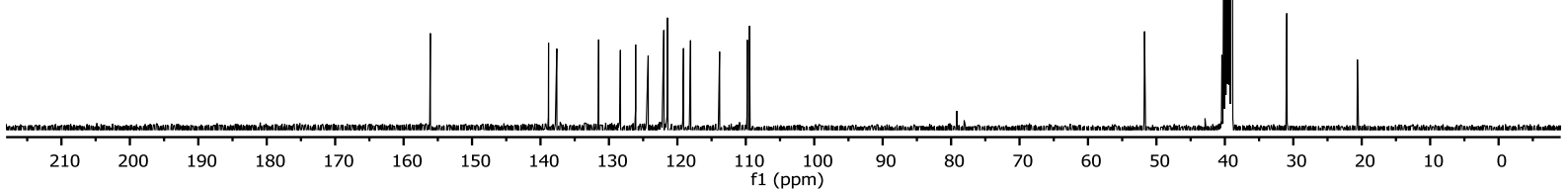



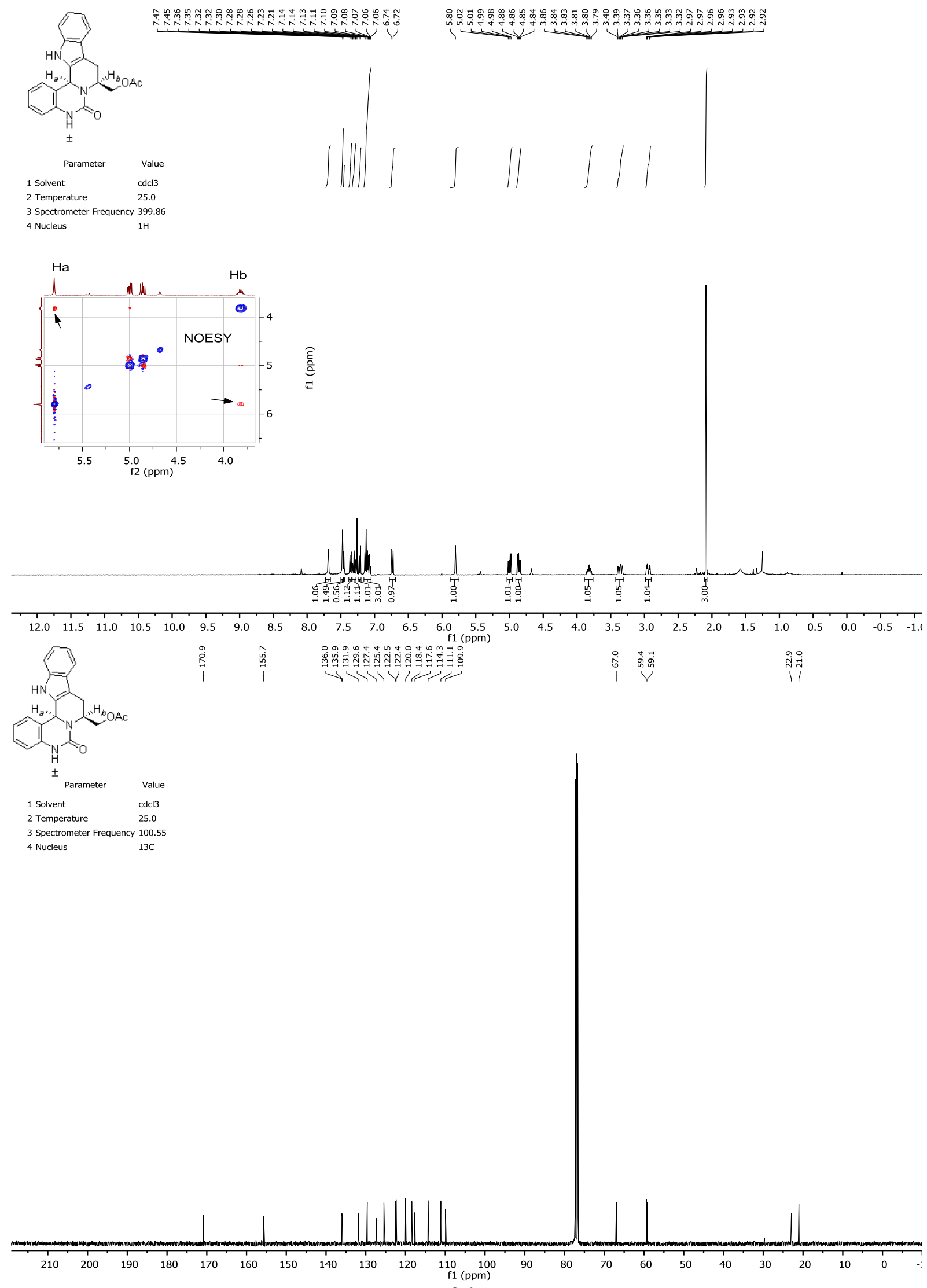

26a 

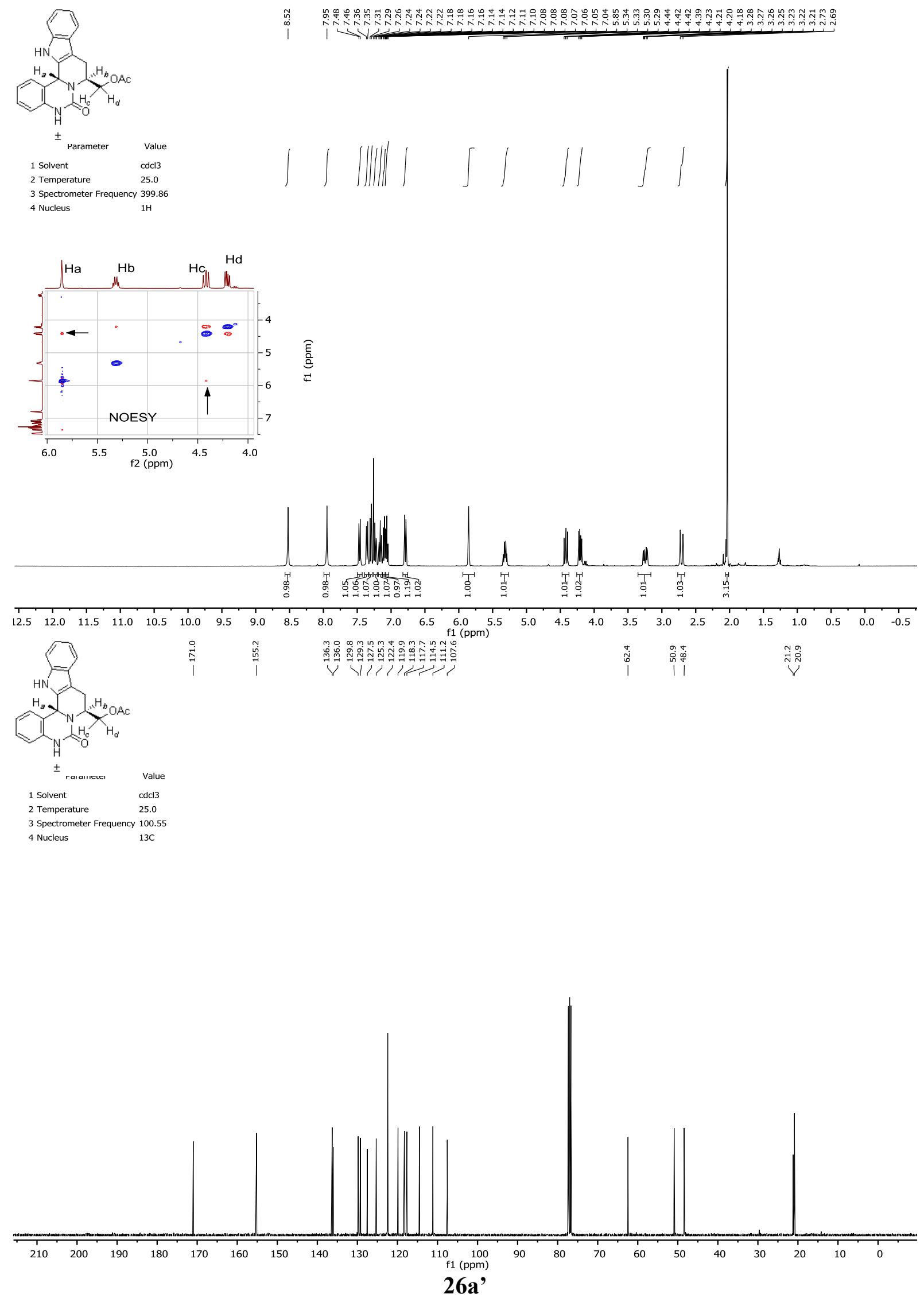


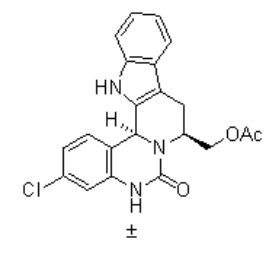

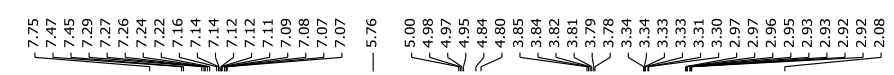
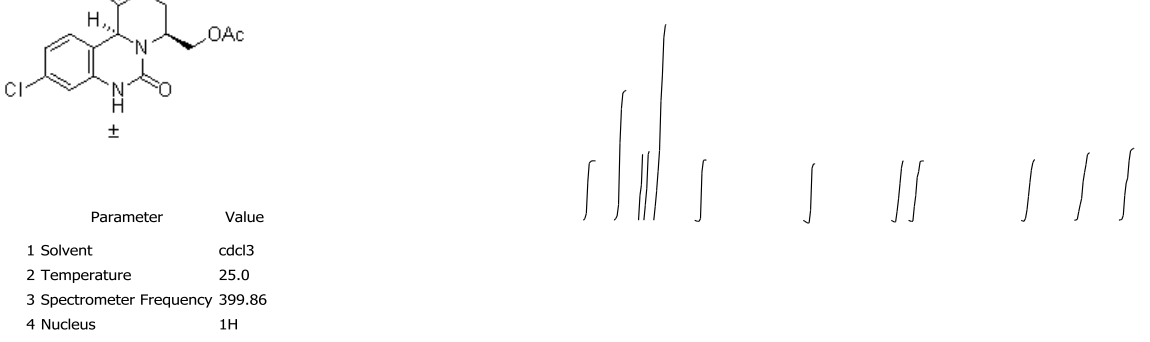

2 Temperature $\quad 25.0$
3 Spectrometer Frequency 399.86

4 Nucleus
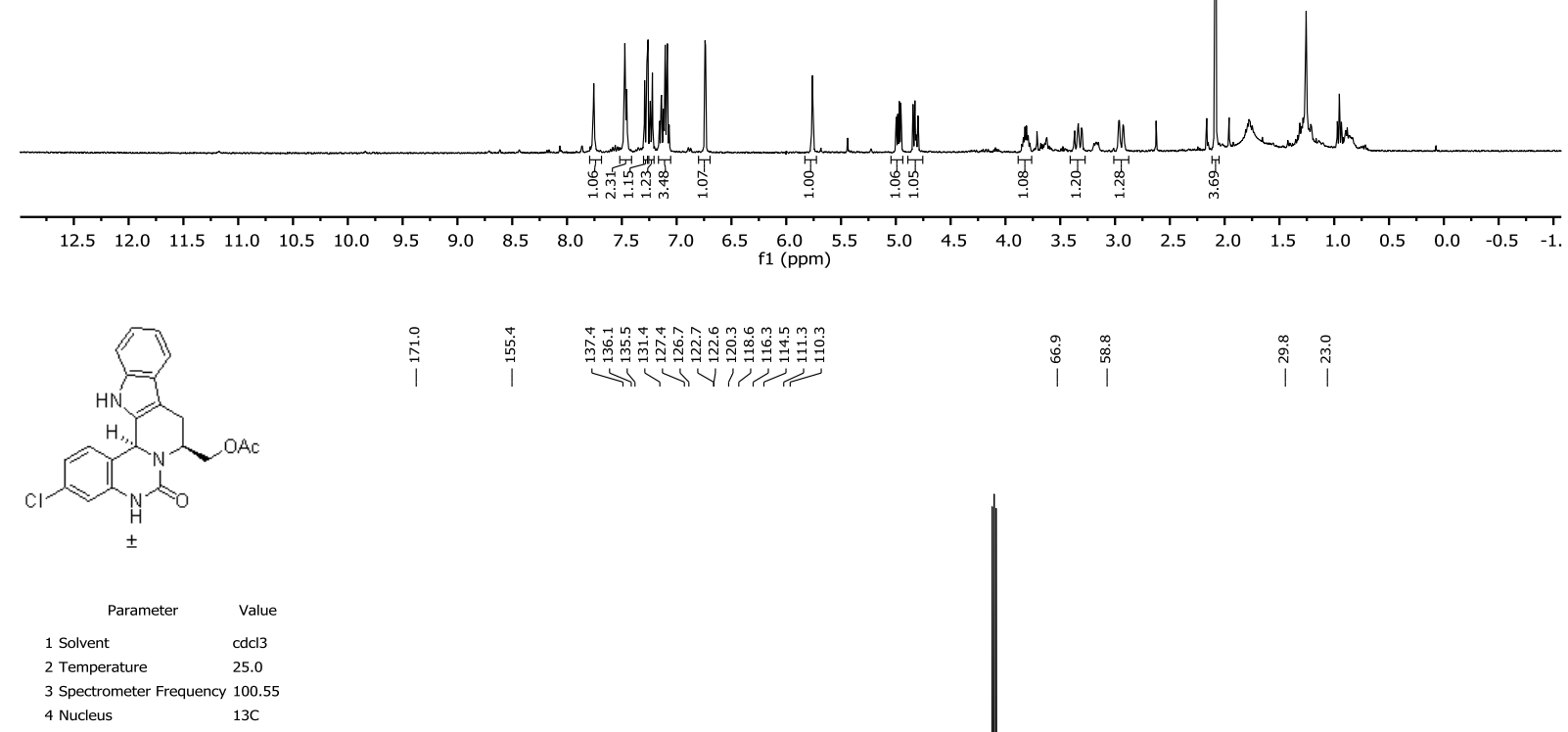

$$
4 \text { Nucleus } \quad 13 \mathrm{C}
$$

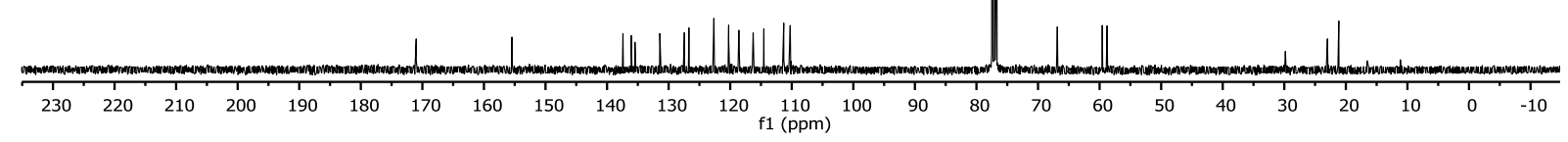



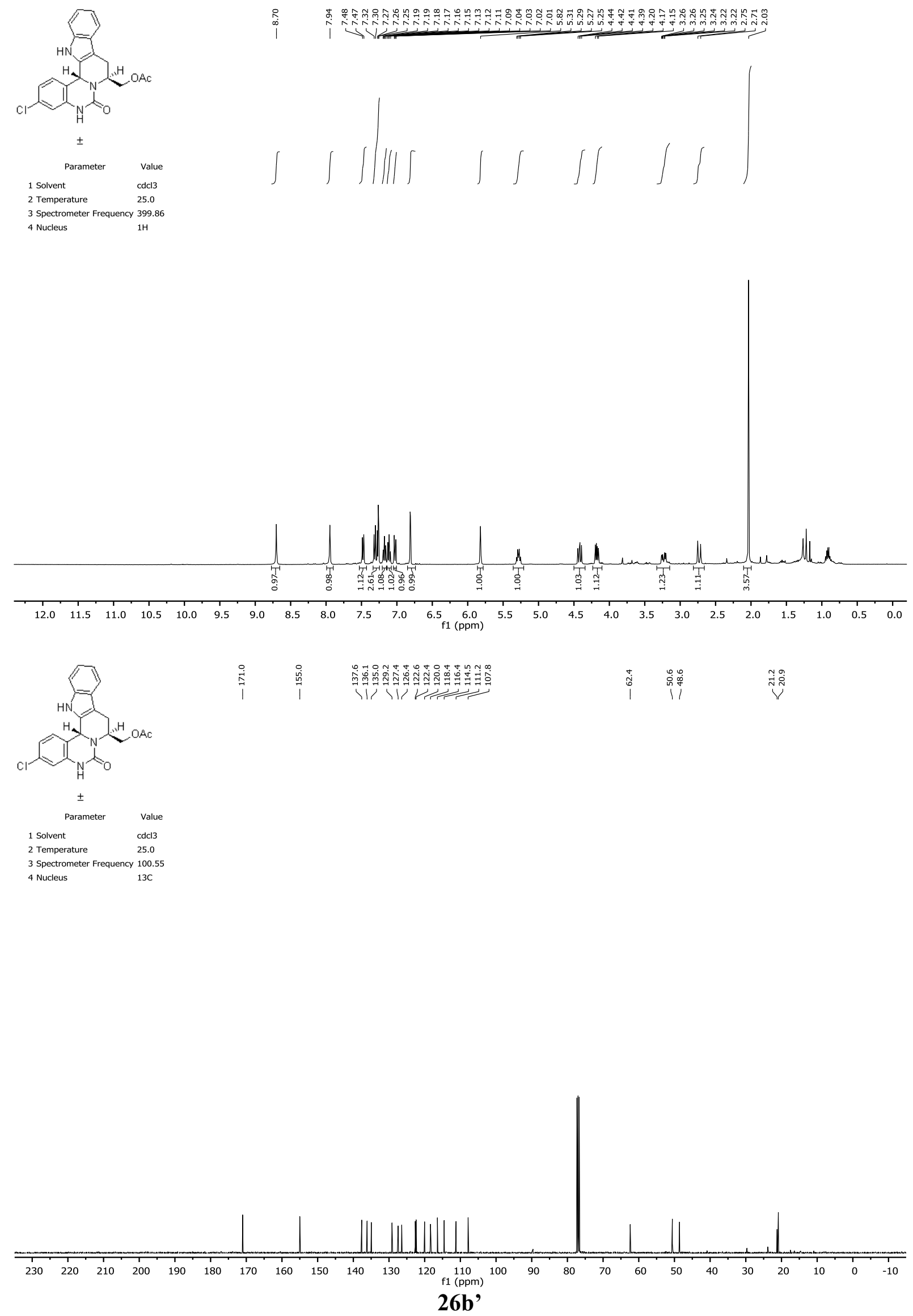
(N)

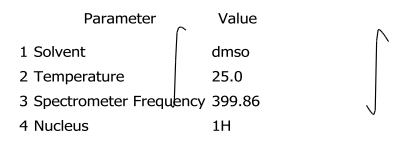

$\iiint \int$

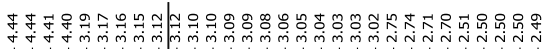

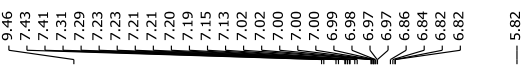

$4 \mathrm{~F}$

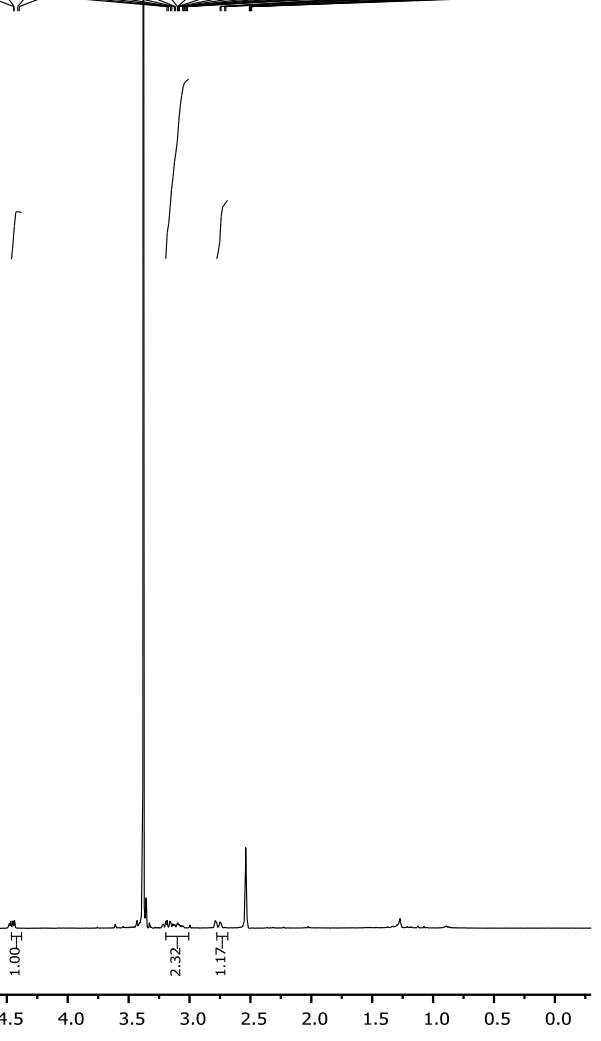

舟

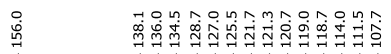

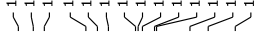

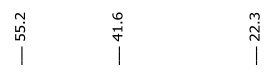

$\begin{array}{lc}\quad \text { Parameter } & \text { Value } \\ 1 \text { Solvent } & \text { dmso } \\ 2 \text { Temperature } & 25.0 \\ 3 \text { Spectrometer Frequency } 100.56 \\ \begin{array}{ll}4 \text { Nucleus } & 13 \mathrm{C}\end{array}\end{array}$

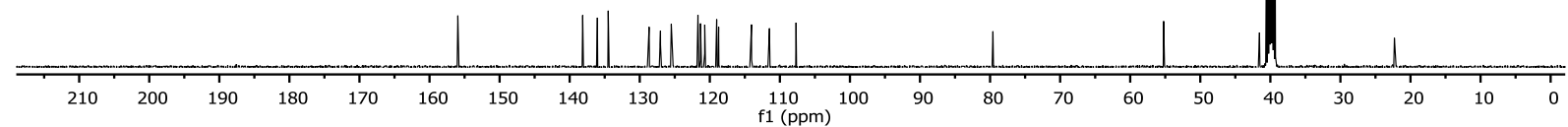
27a 

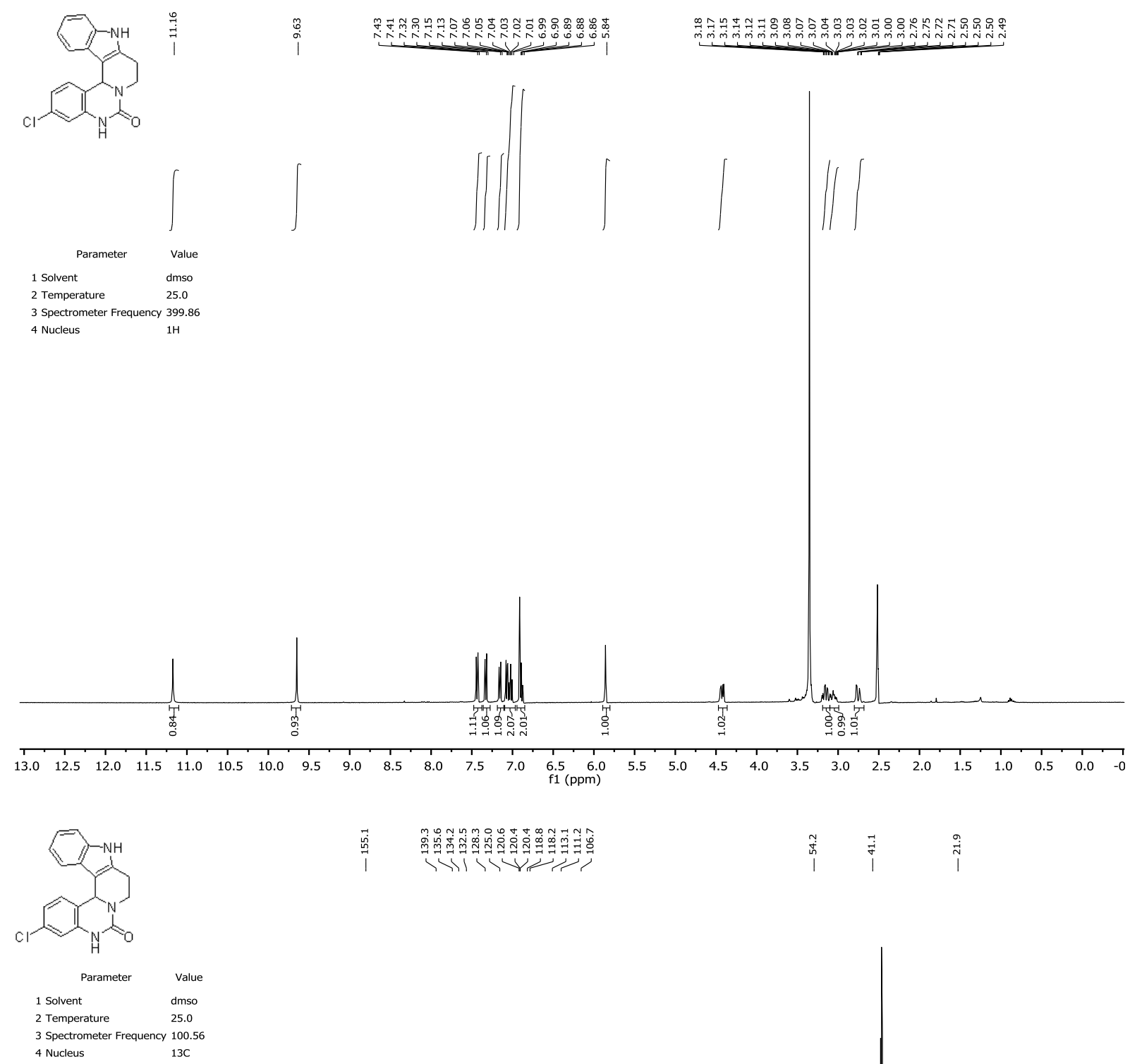

|

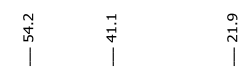

4 Nucleus

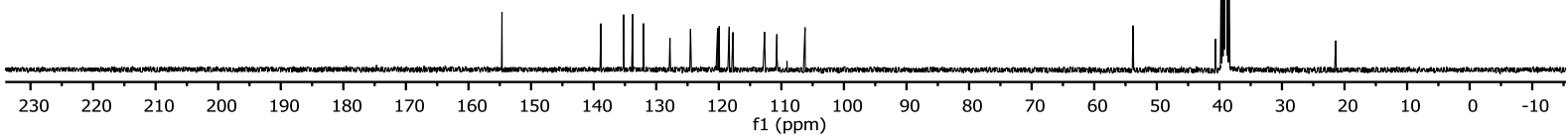



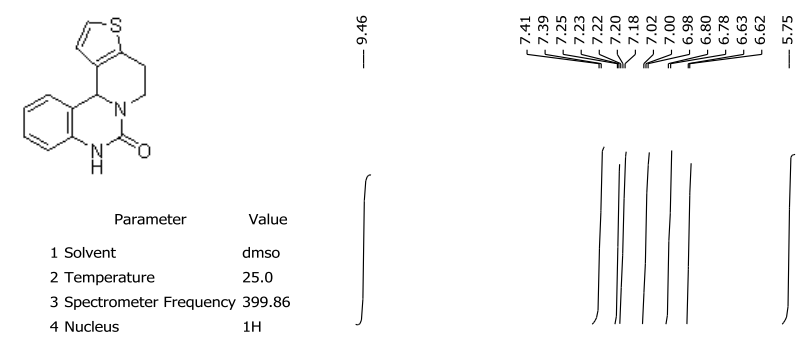

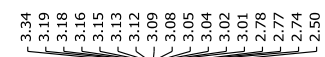
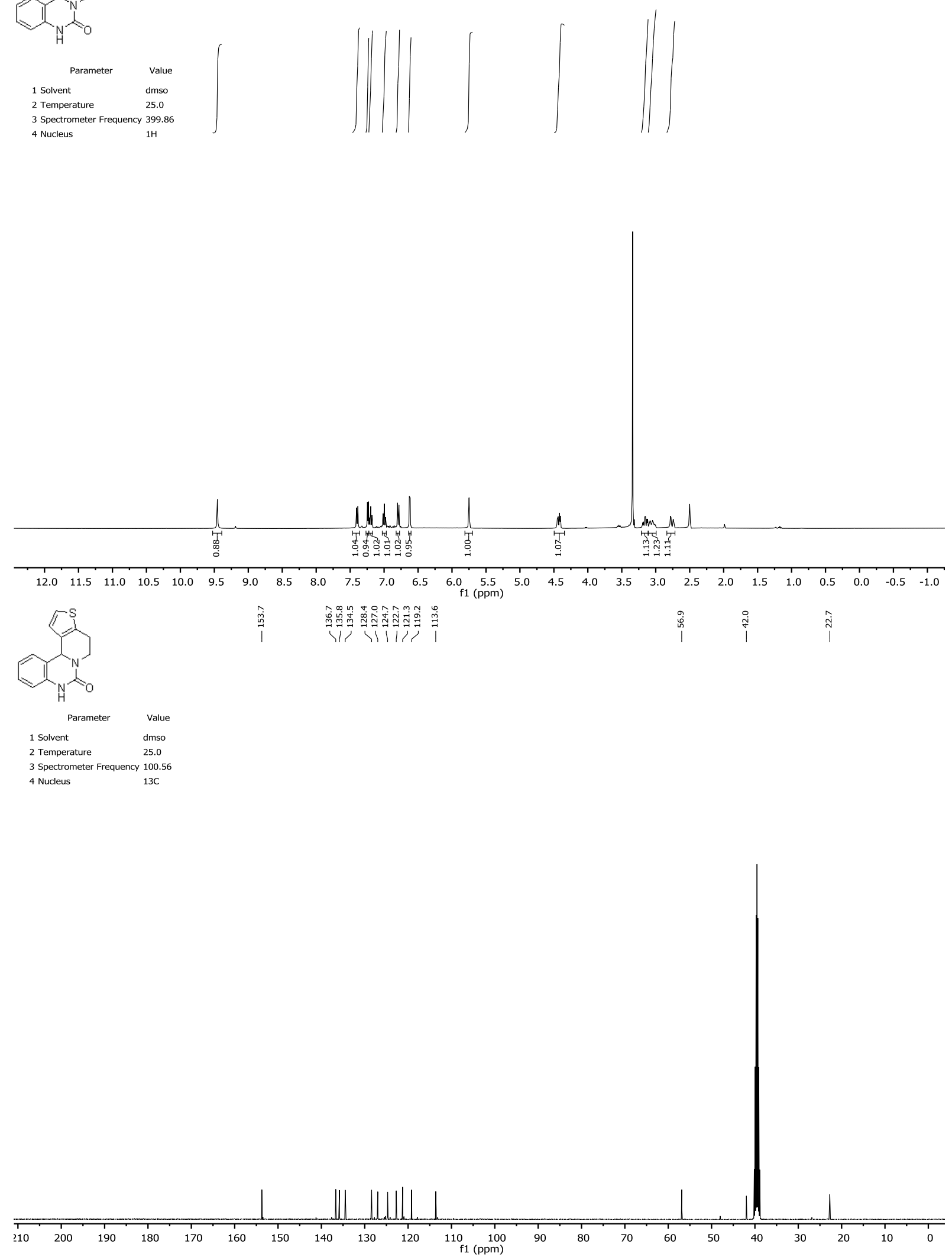

28a 


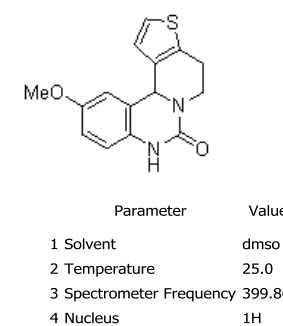

$\underbrace{\text { non }}$

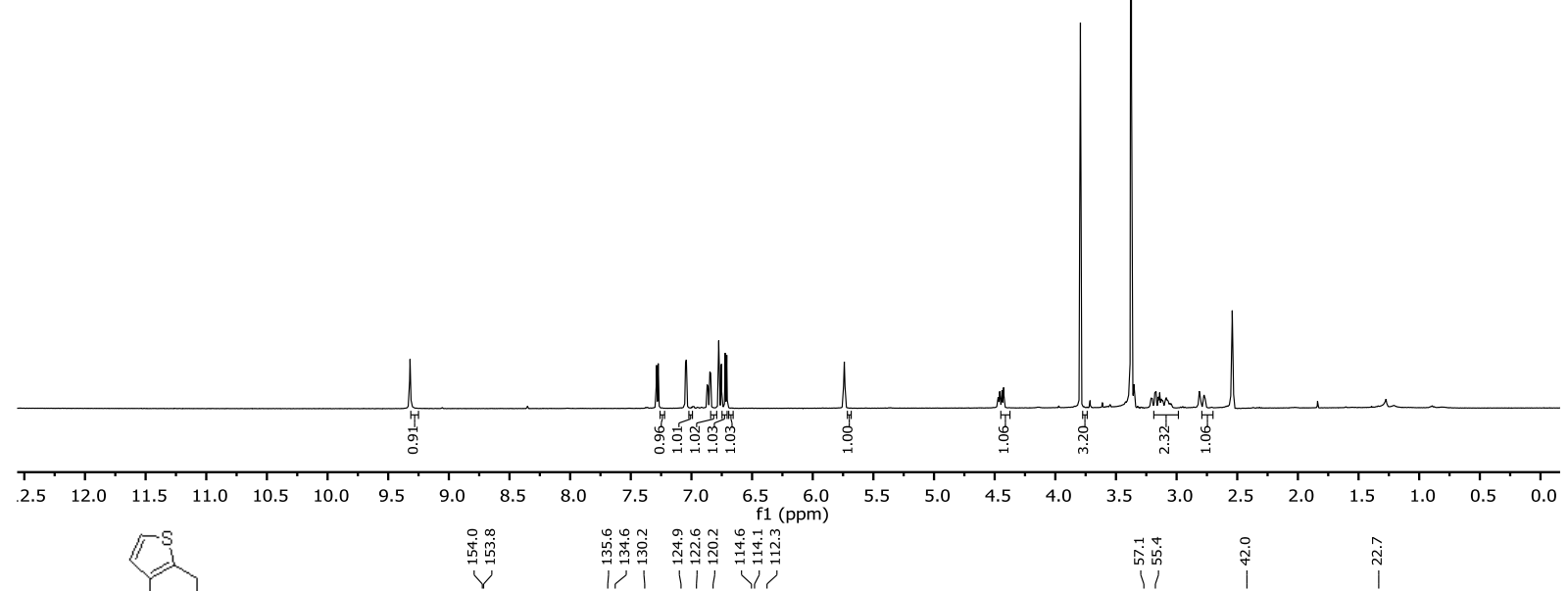

MeO

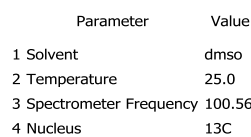

4 Nucleus $\quad 13 \mathrm{C}$

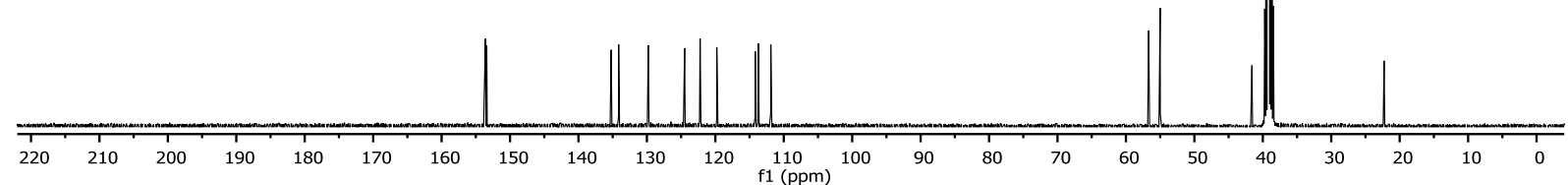

28b 

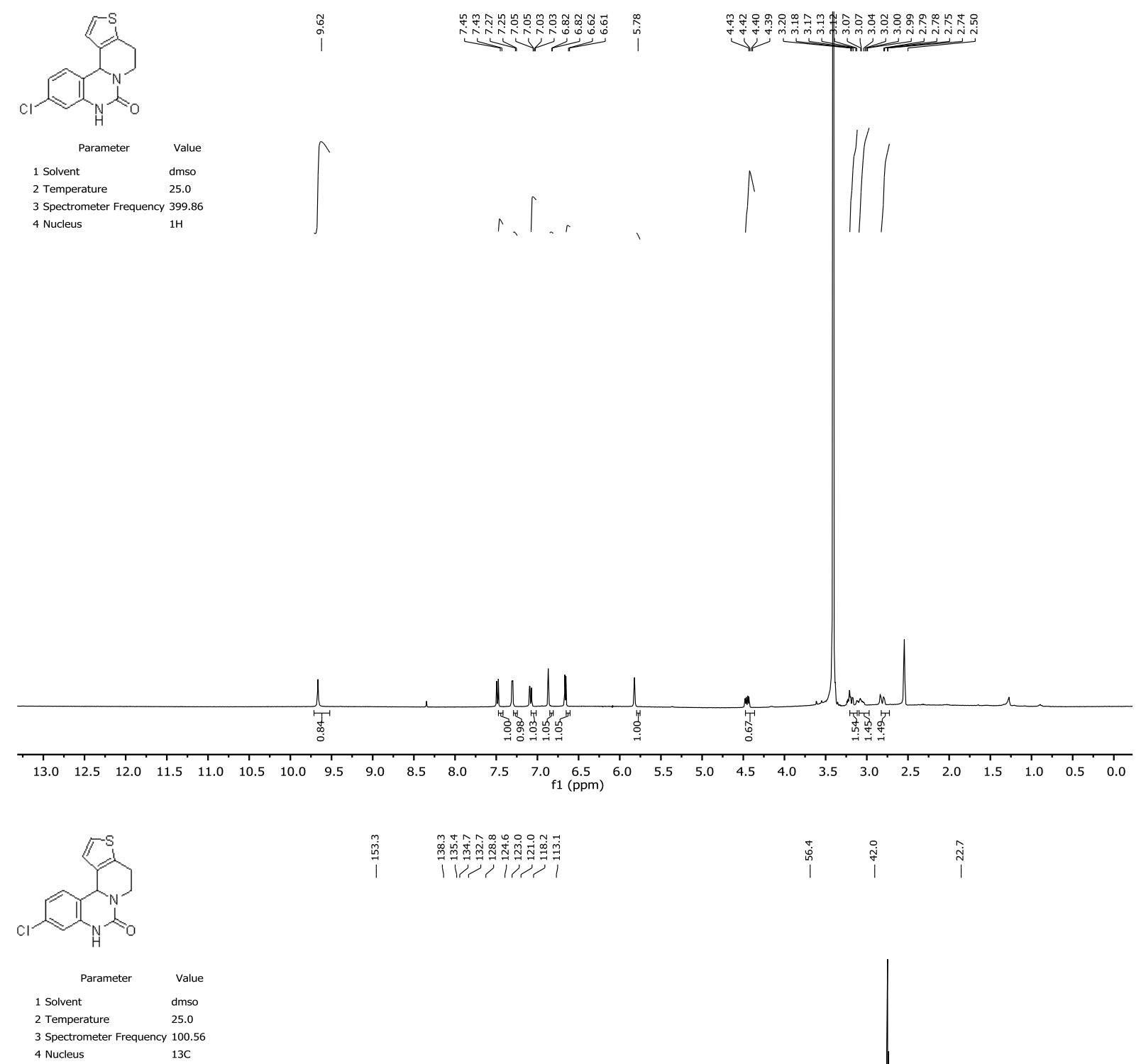

| l|

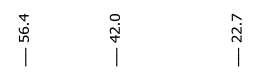

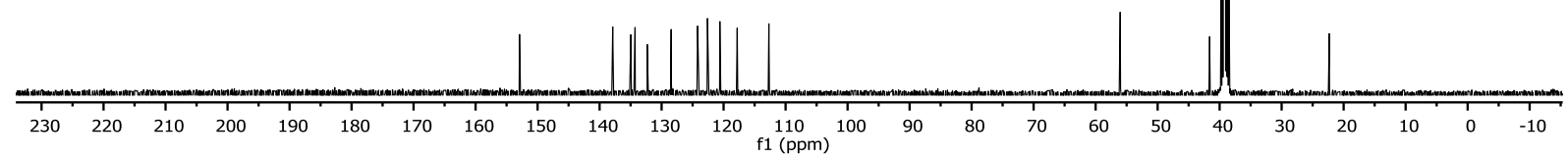

$28 c$ 

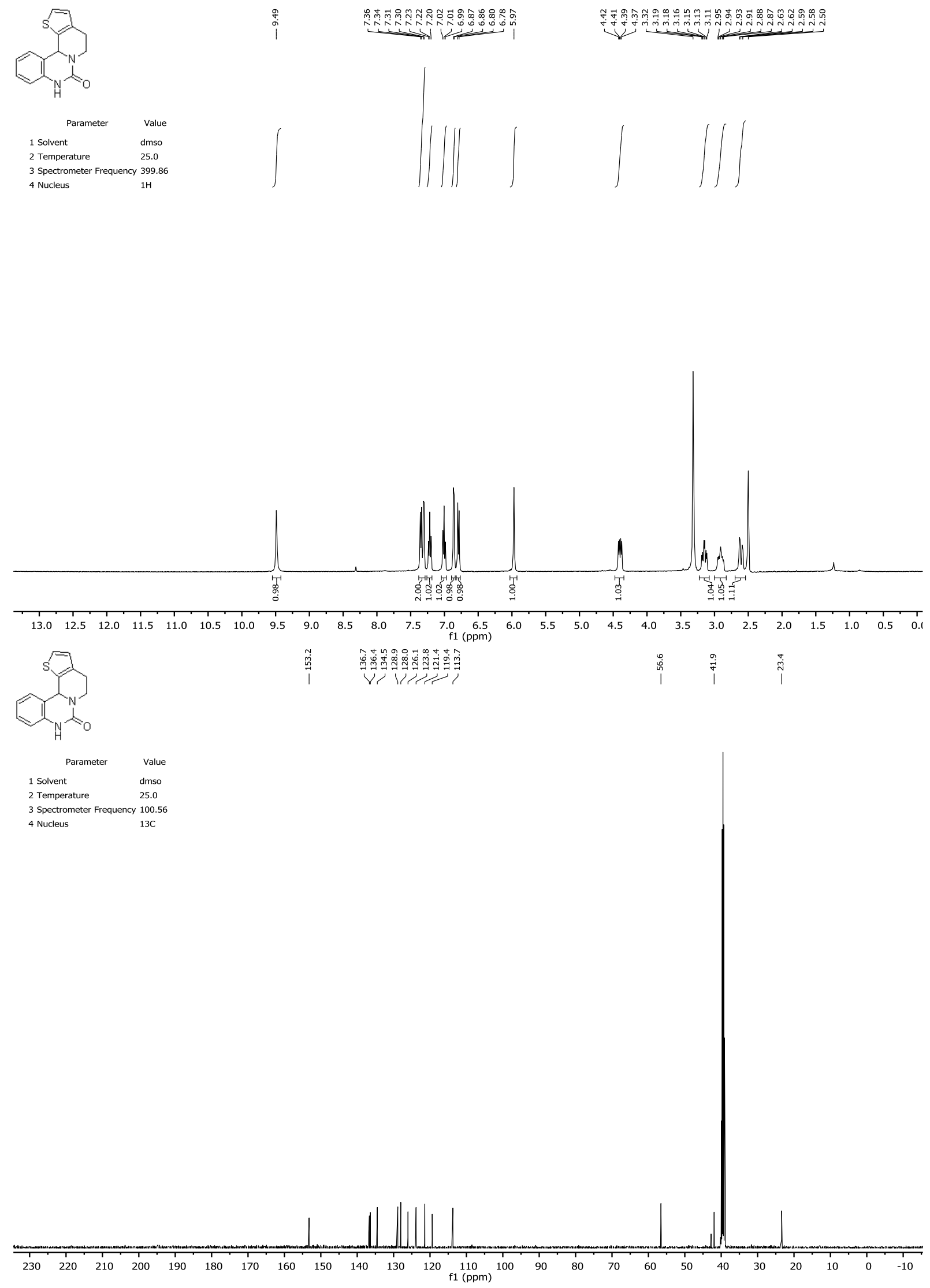

29a 

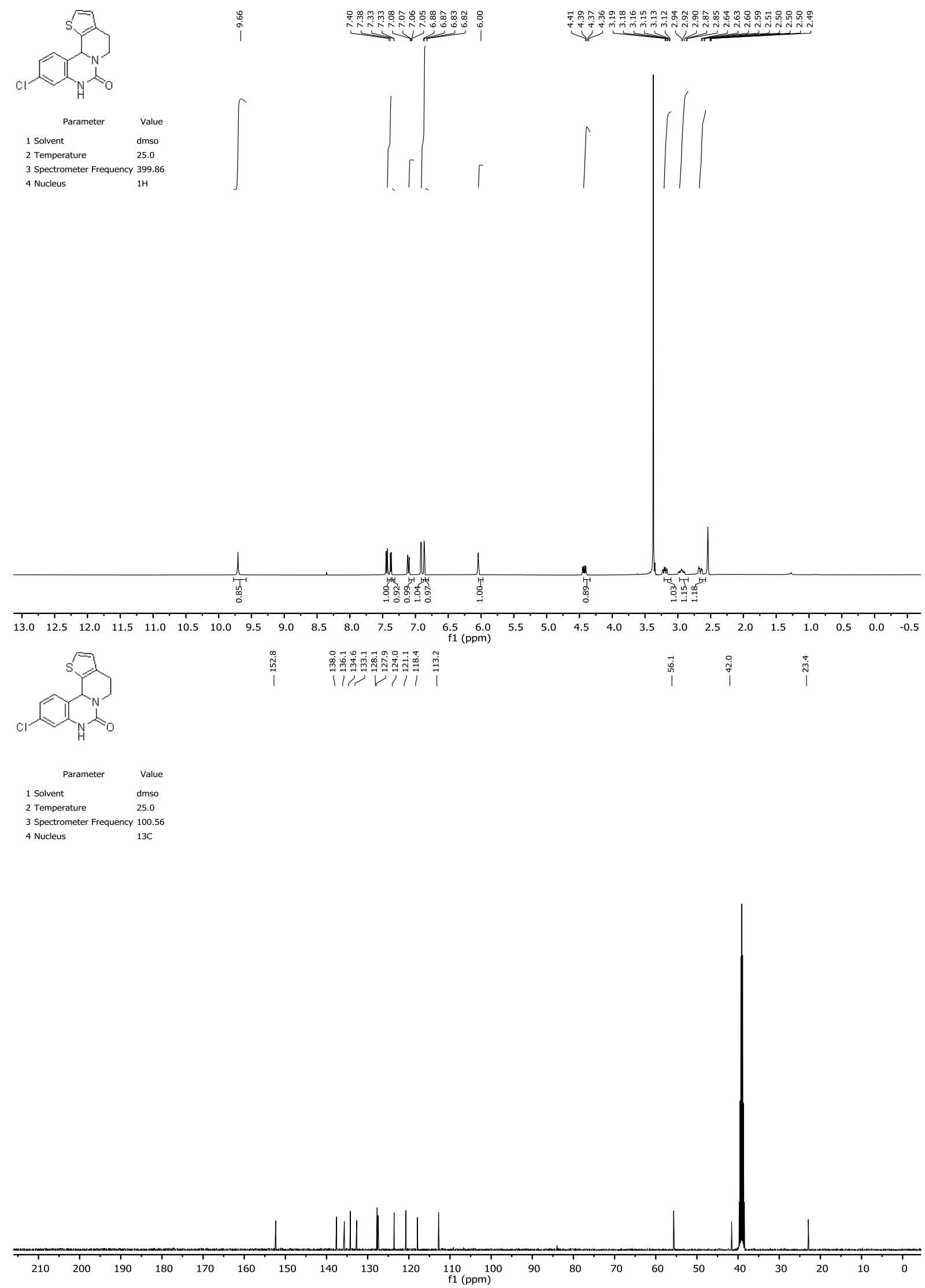

29b 


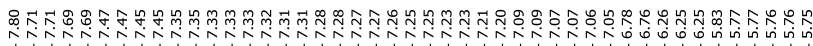
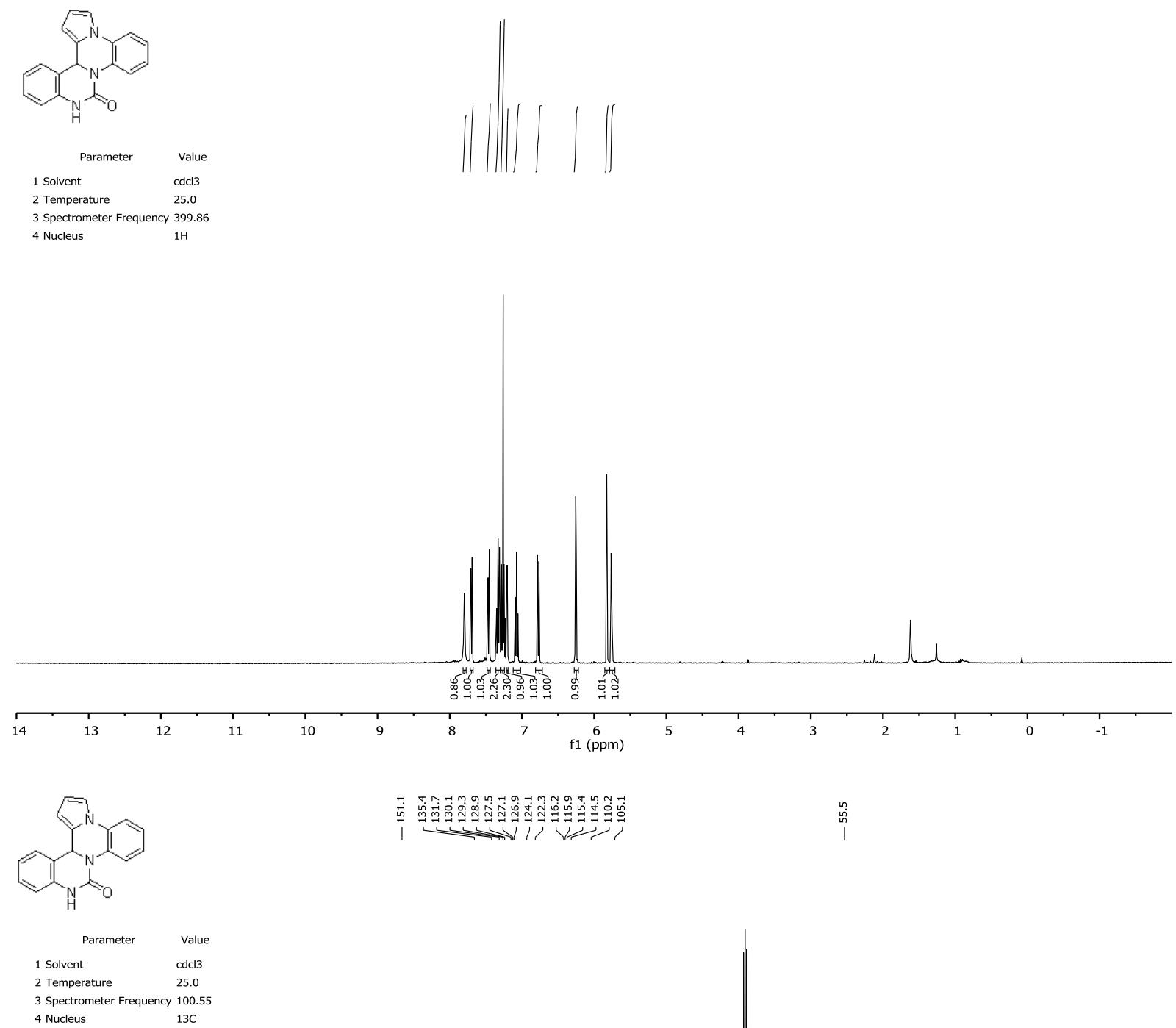

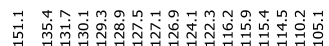

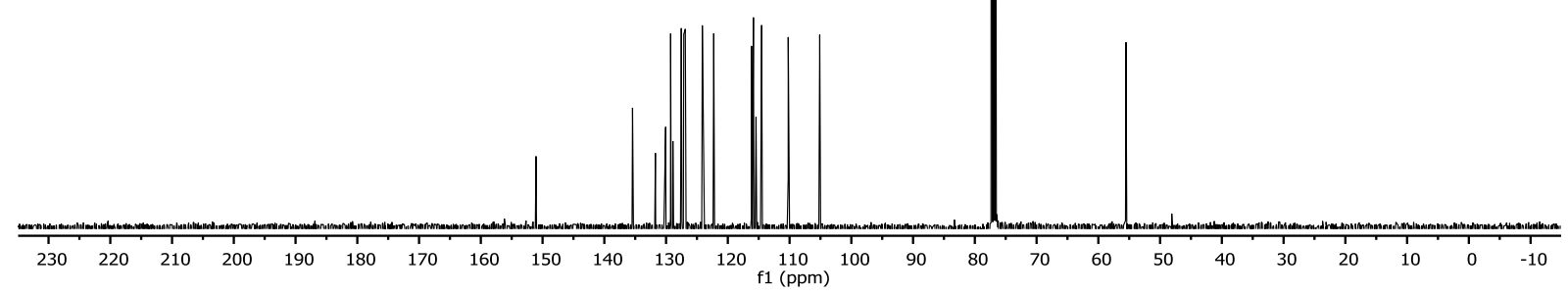

30a 

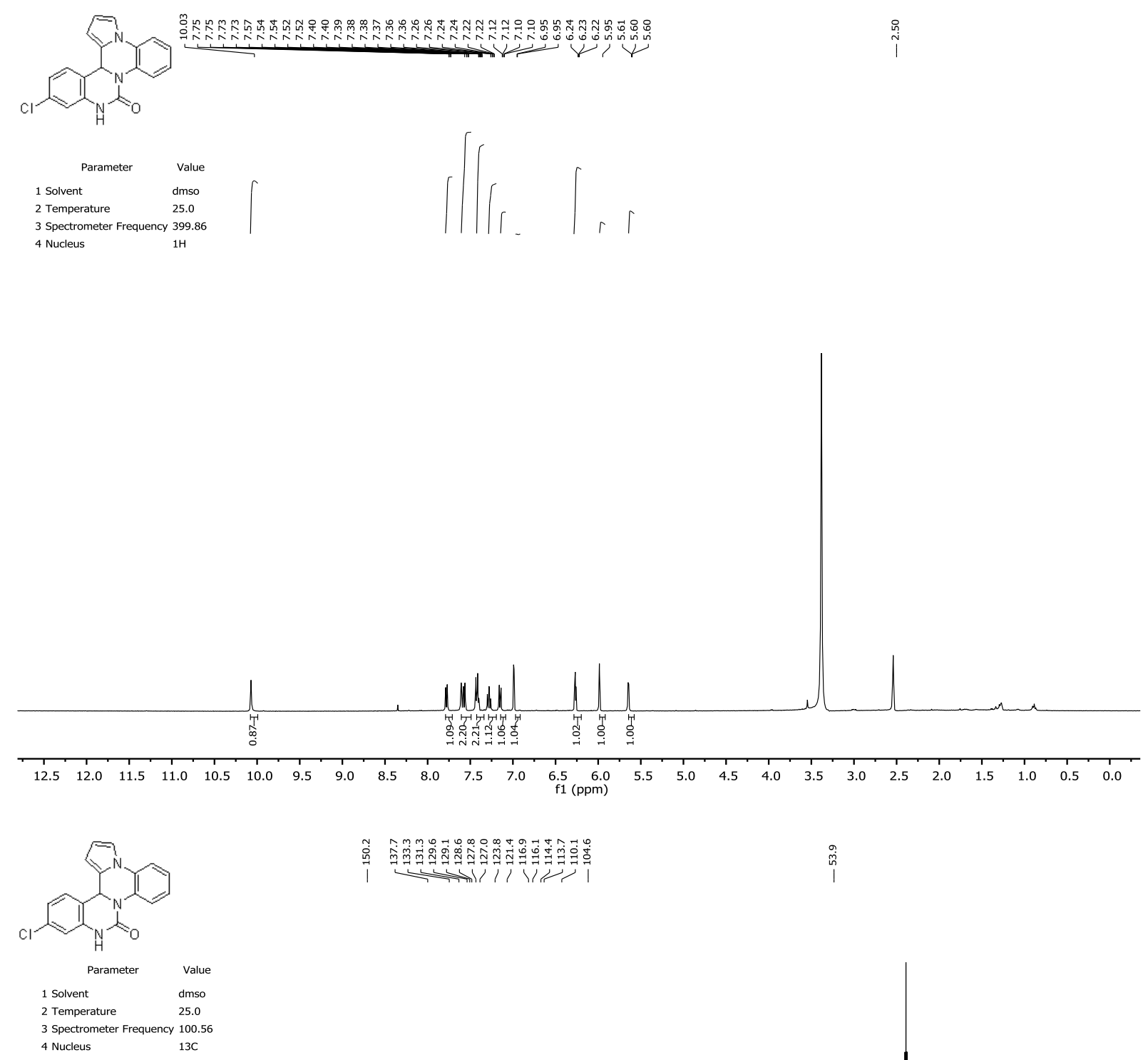

|

$\stackrel{\substack{n \\ \text { นn }}}{1}$

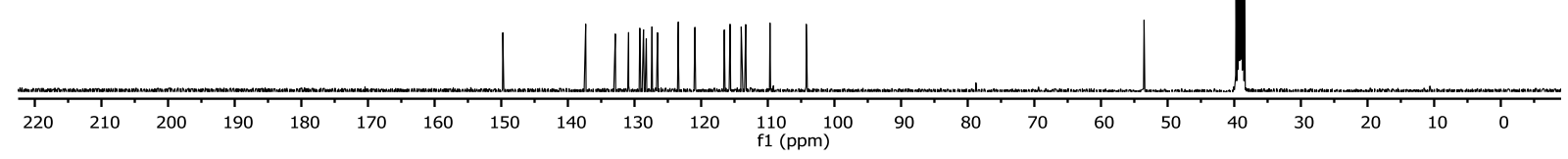
30b 

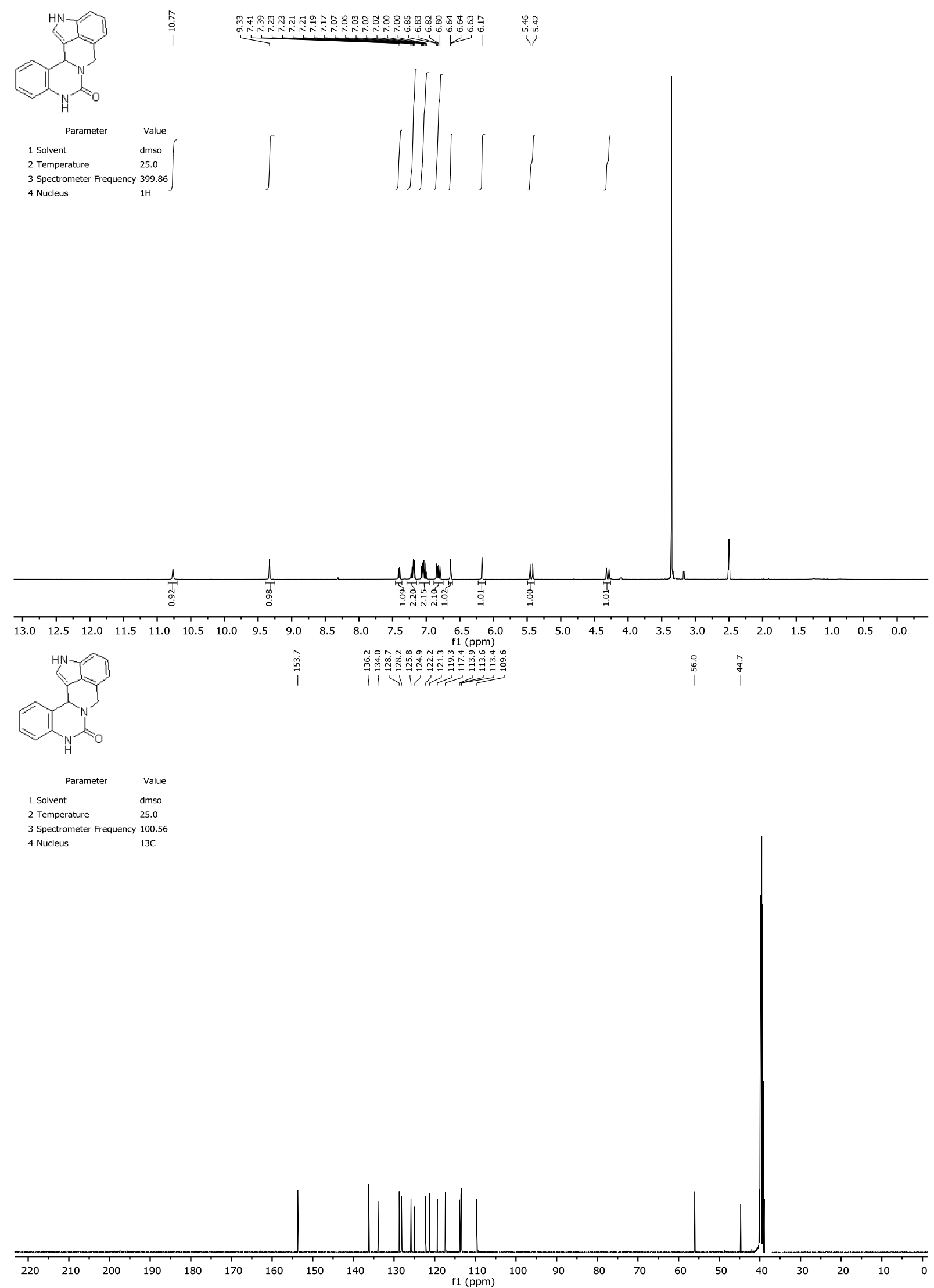

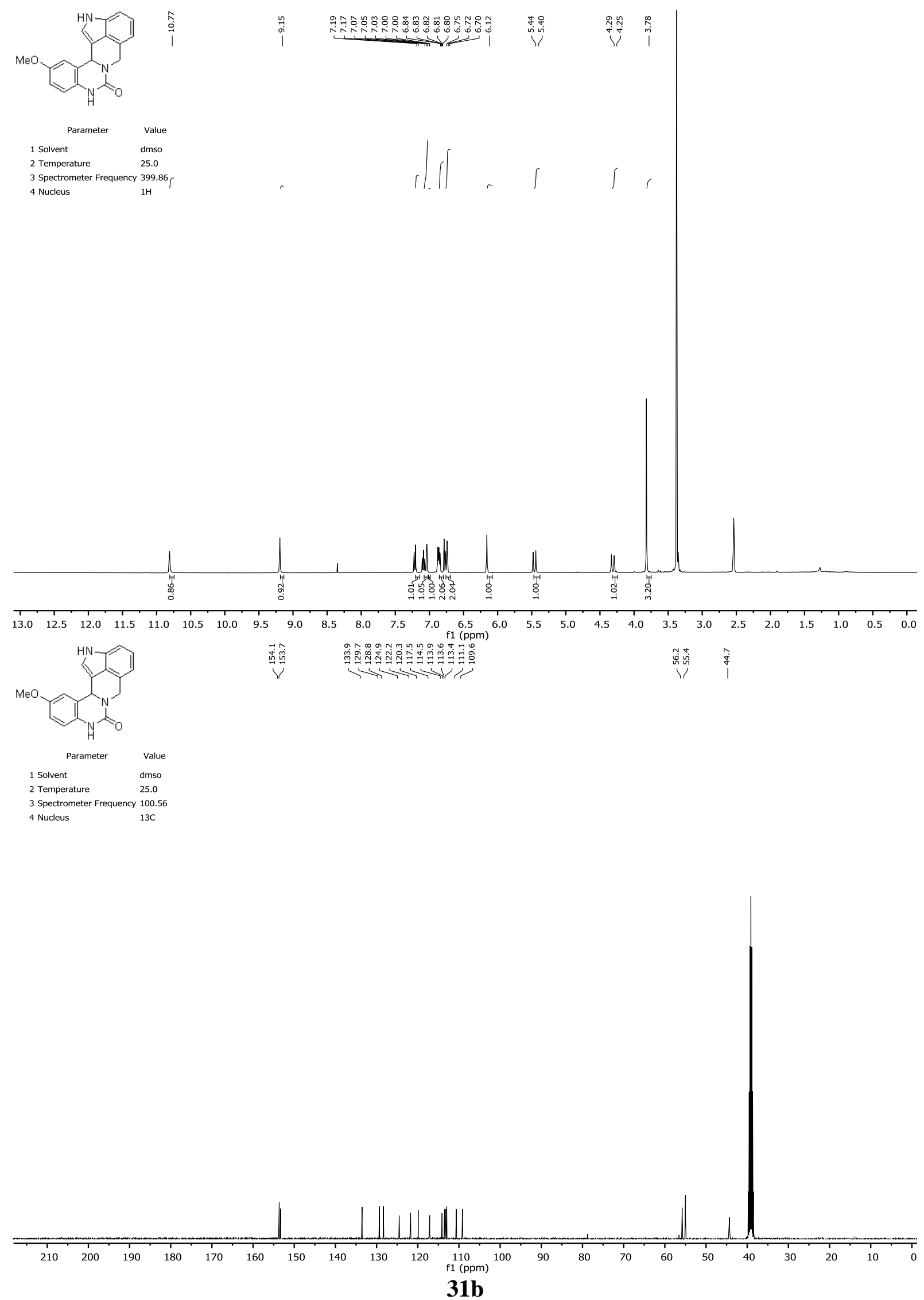

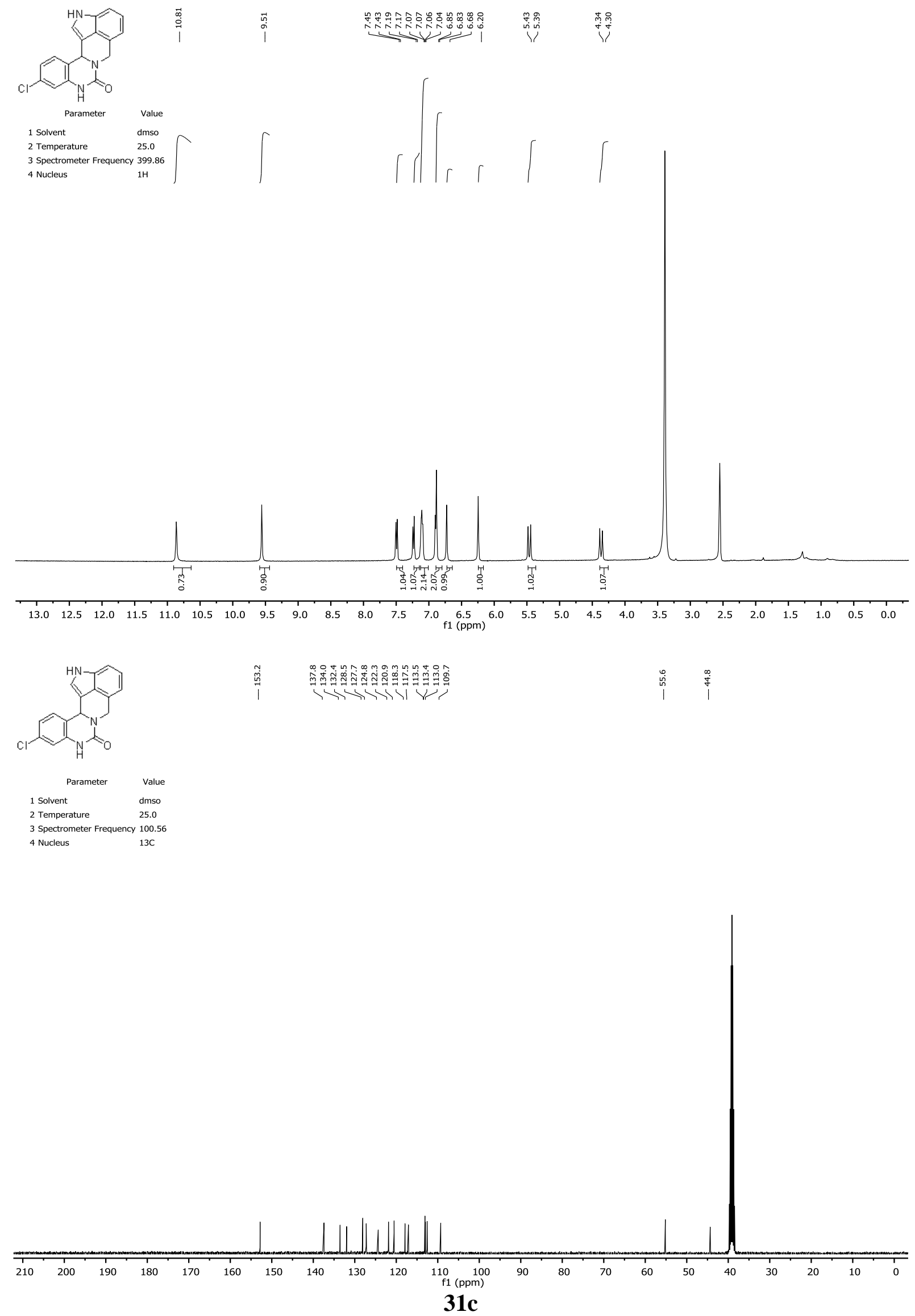

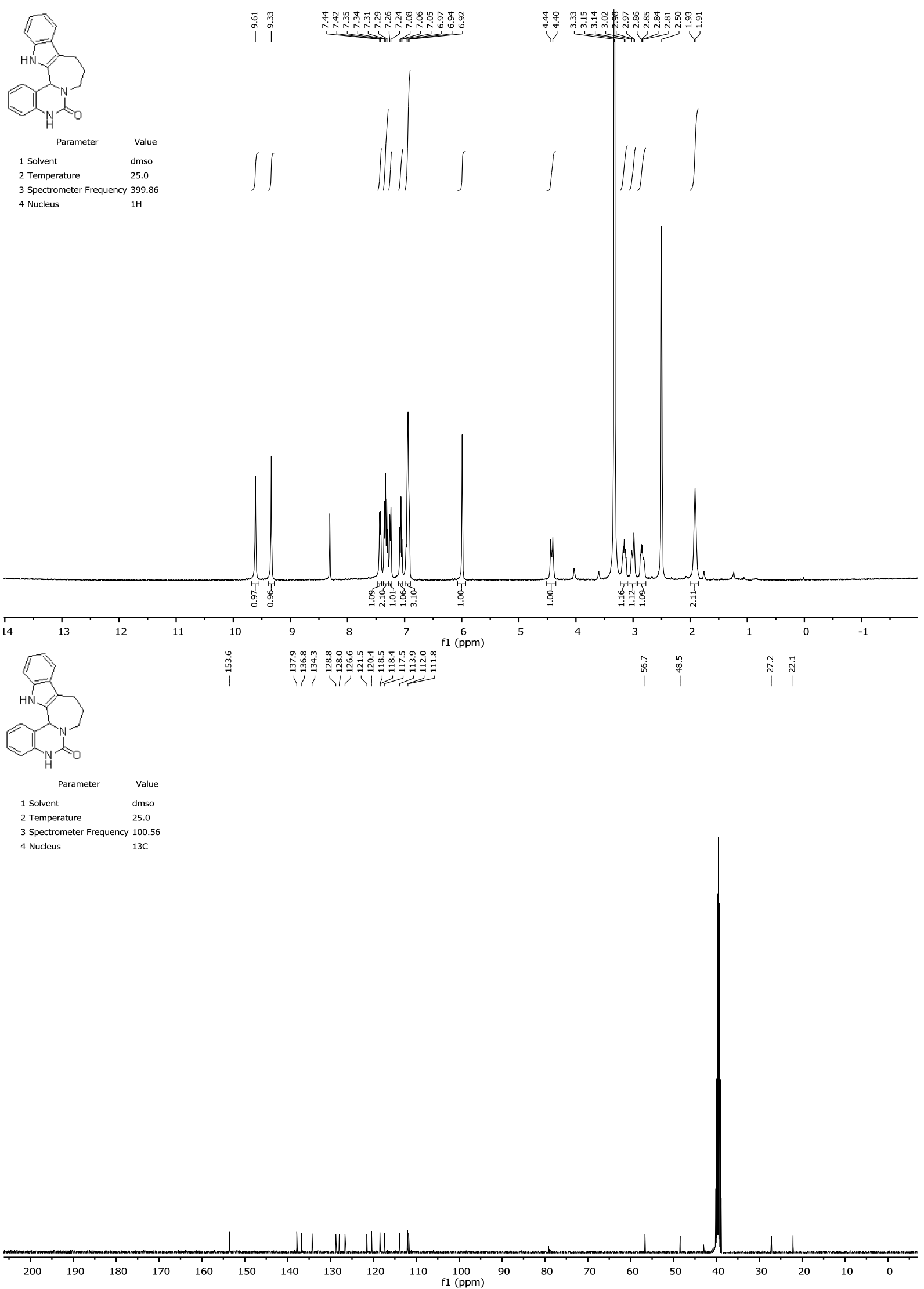


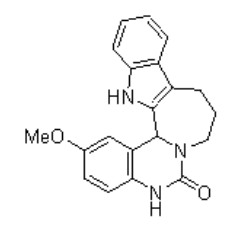

\begin{tabular}{lr}
\multicolumn{1}{c}{ Parameter } & Valu \\
1 Solvent & dmso \\
2 Temperature & 25.0 \\
3 Spectrometer Frequency & 399.8 \\
4 Nucleus & $1 \mathrm{H}$
\end{tabular}
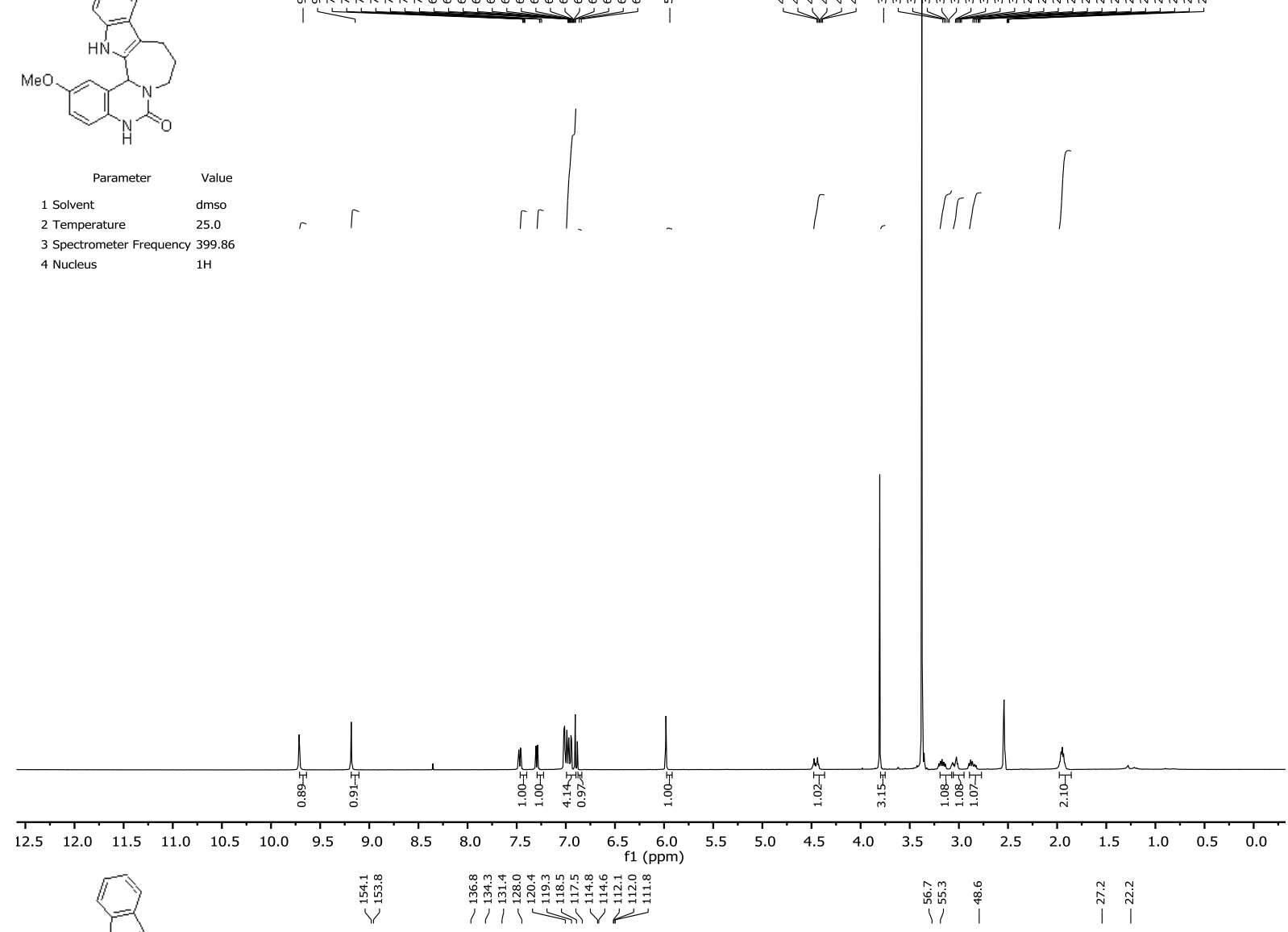

$\mathrm{MeO}$

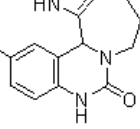

11।

\1।
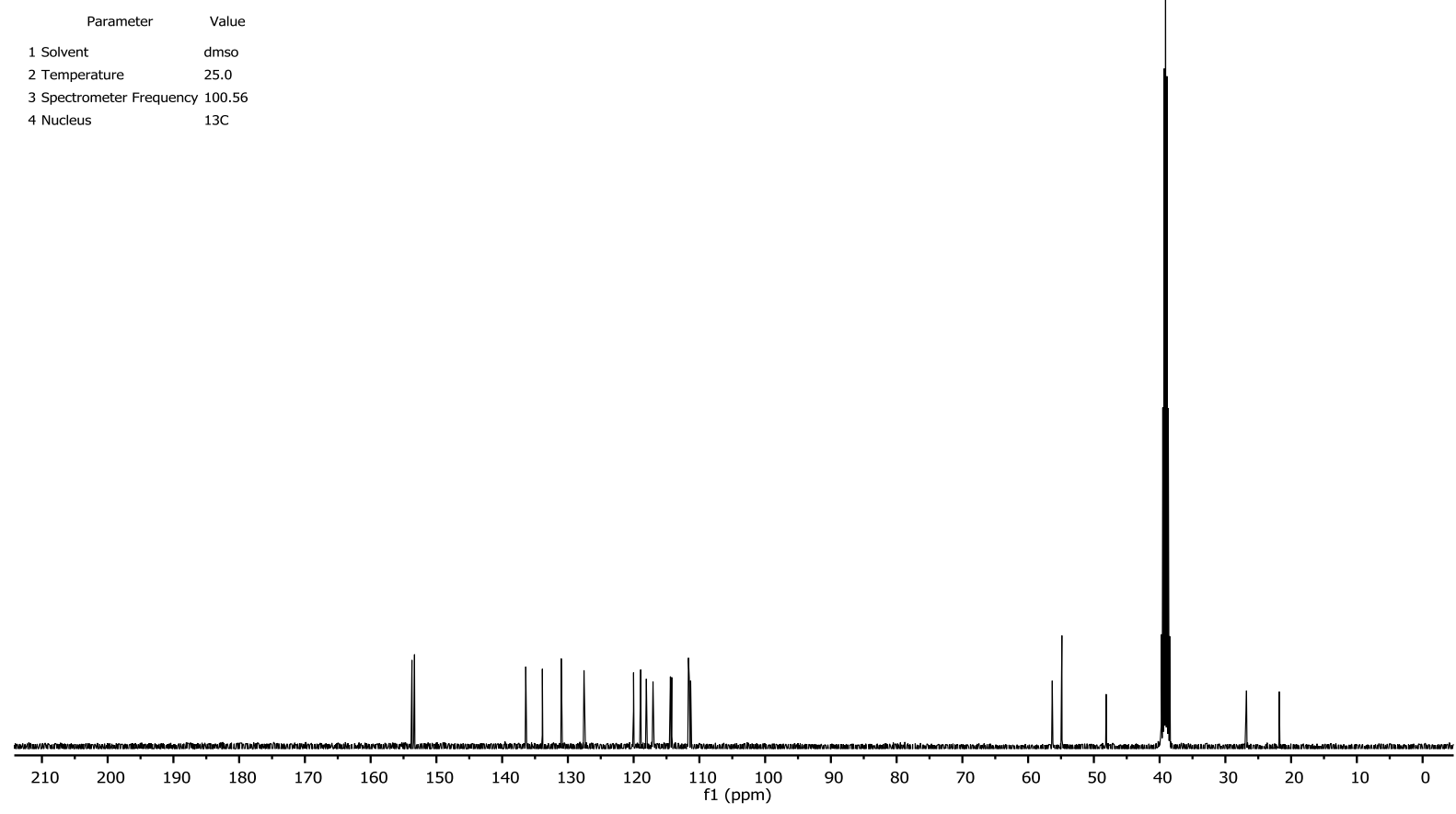

32b 

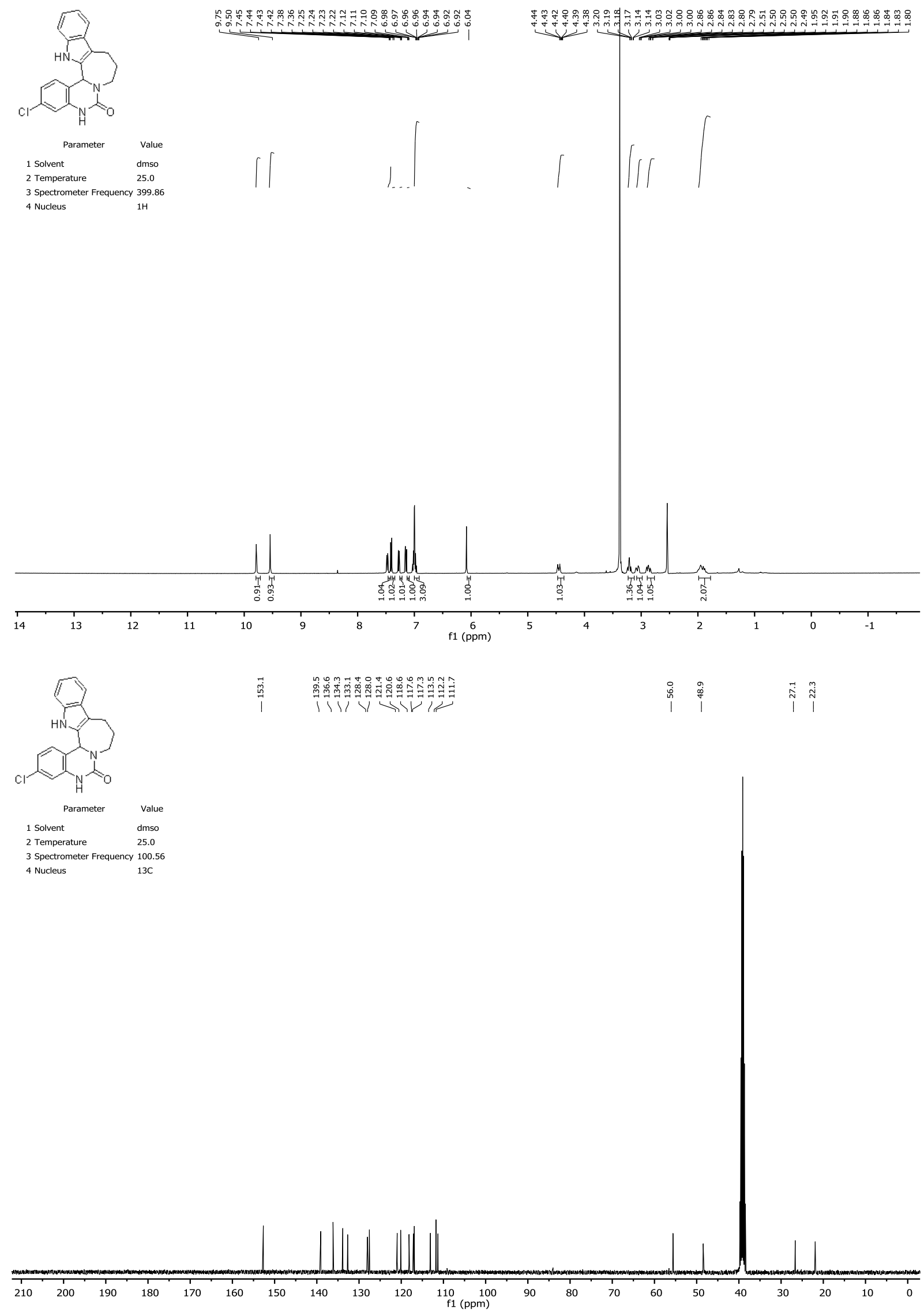

32c 


\section{$\mathrm{MeO}$}

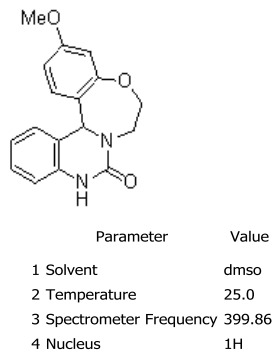

4 Nucleus $\quad 1 \mathrm{H}$

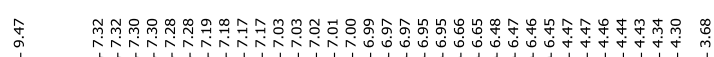

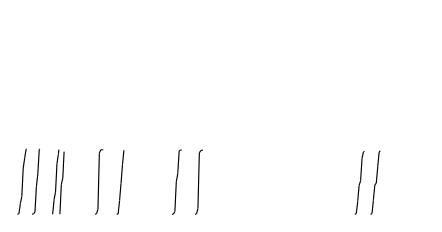

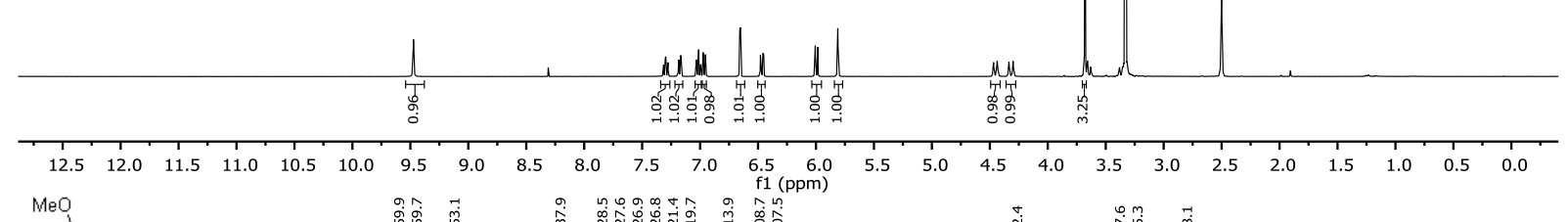

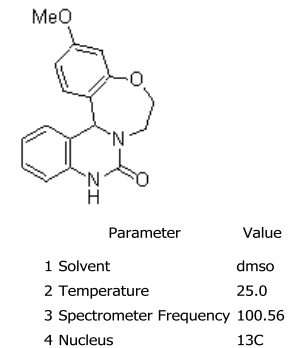

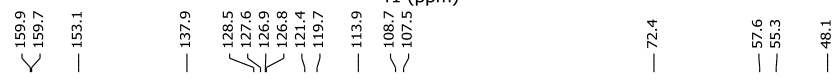

4 Nucleus

$13 \mathrm{C}$

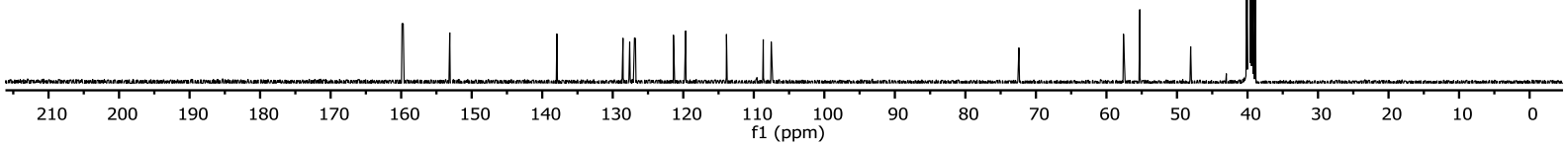

33a 


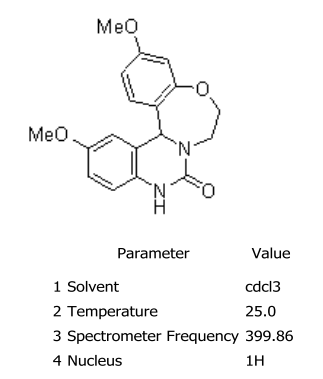

年
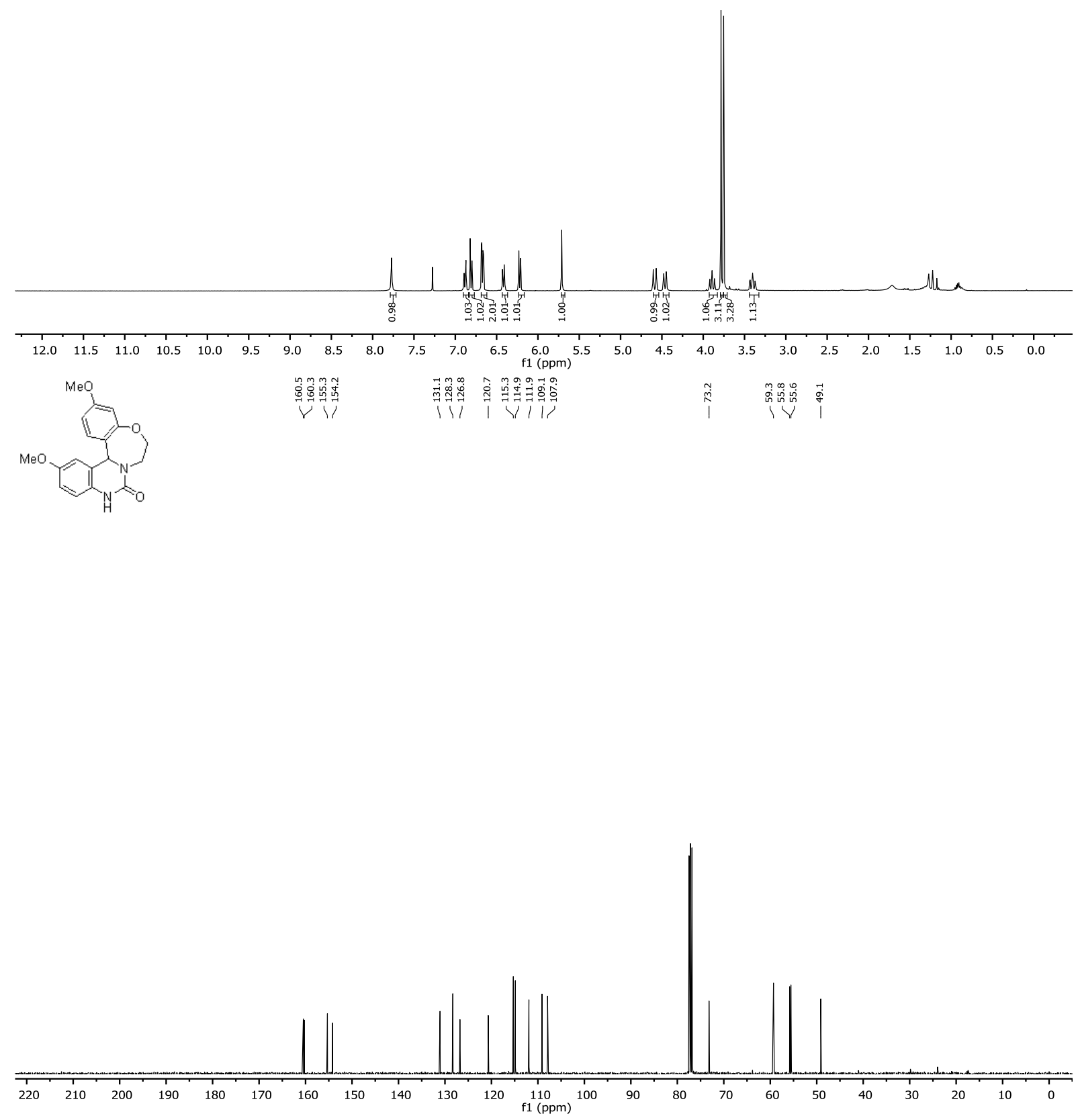

33b 

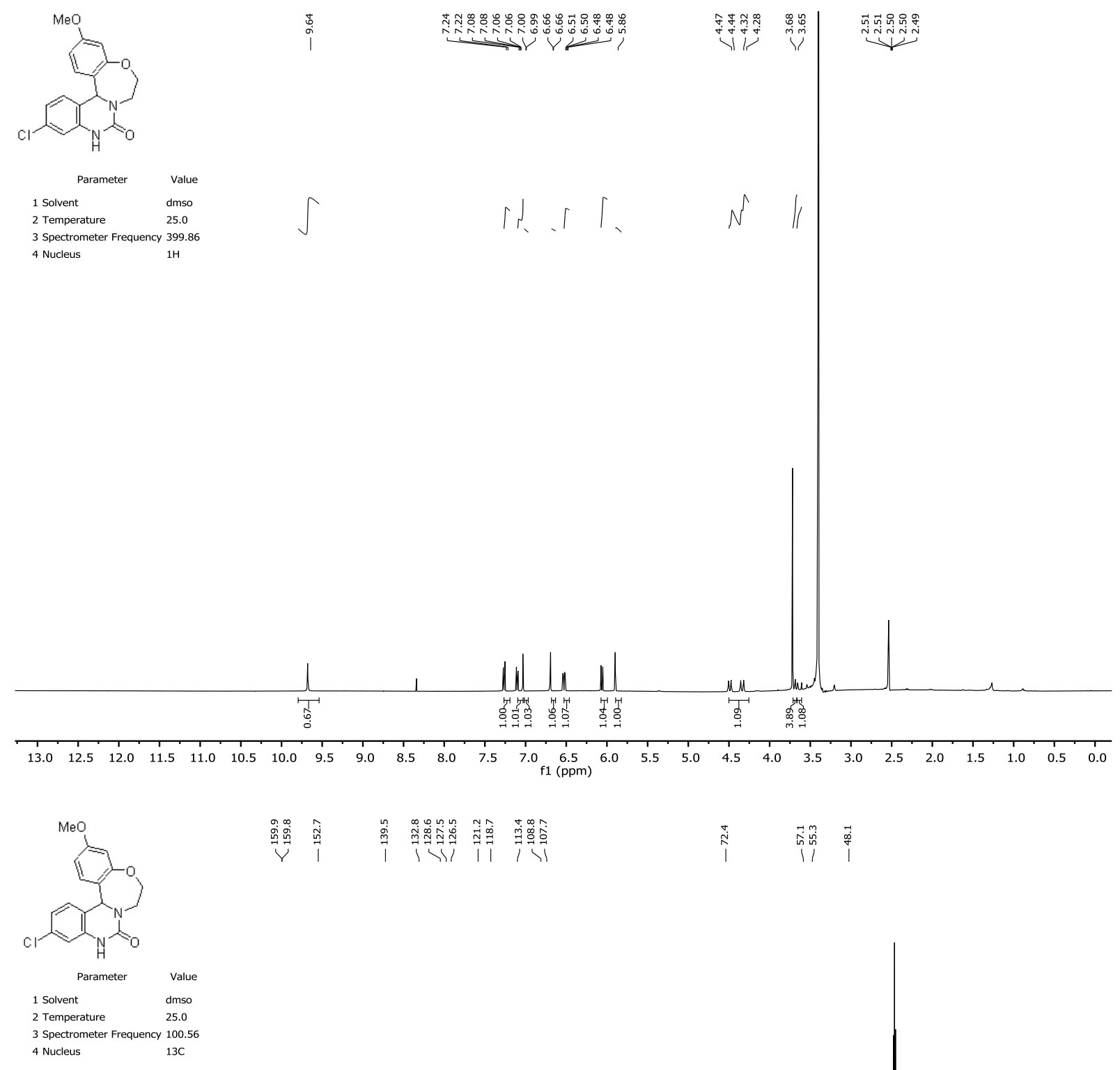

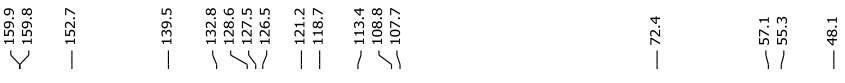

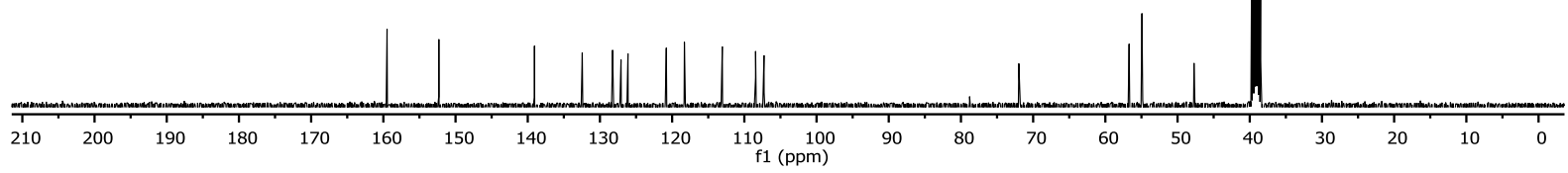

33c 

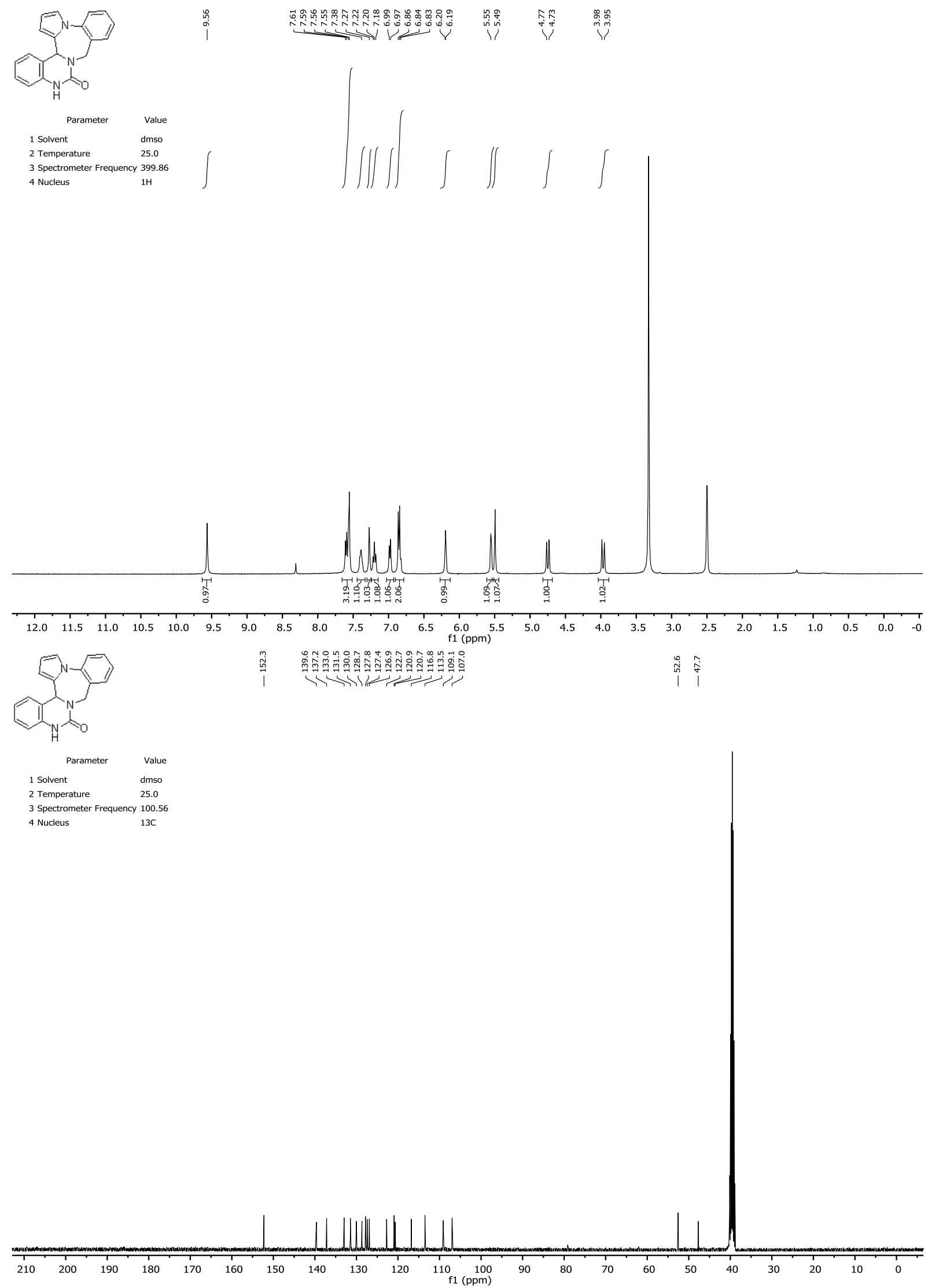

34a 

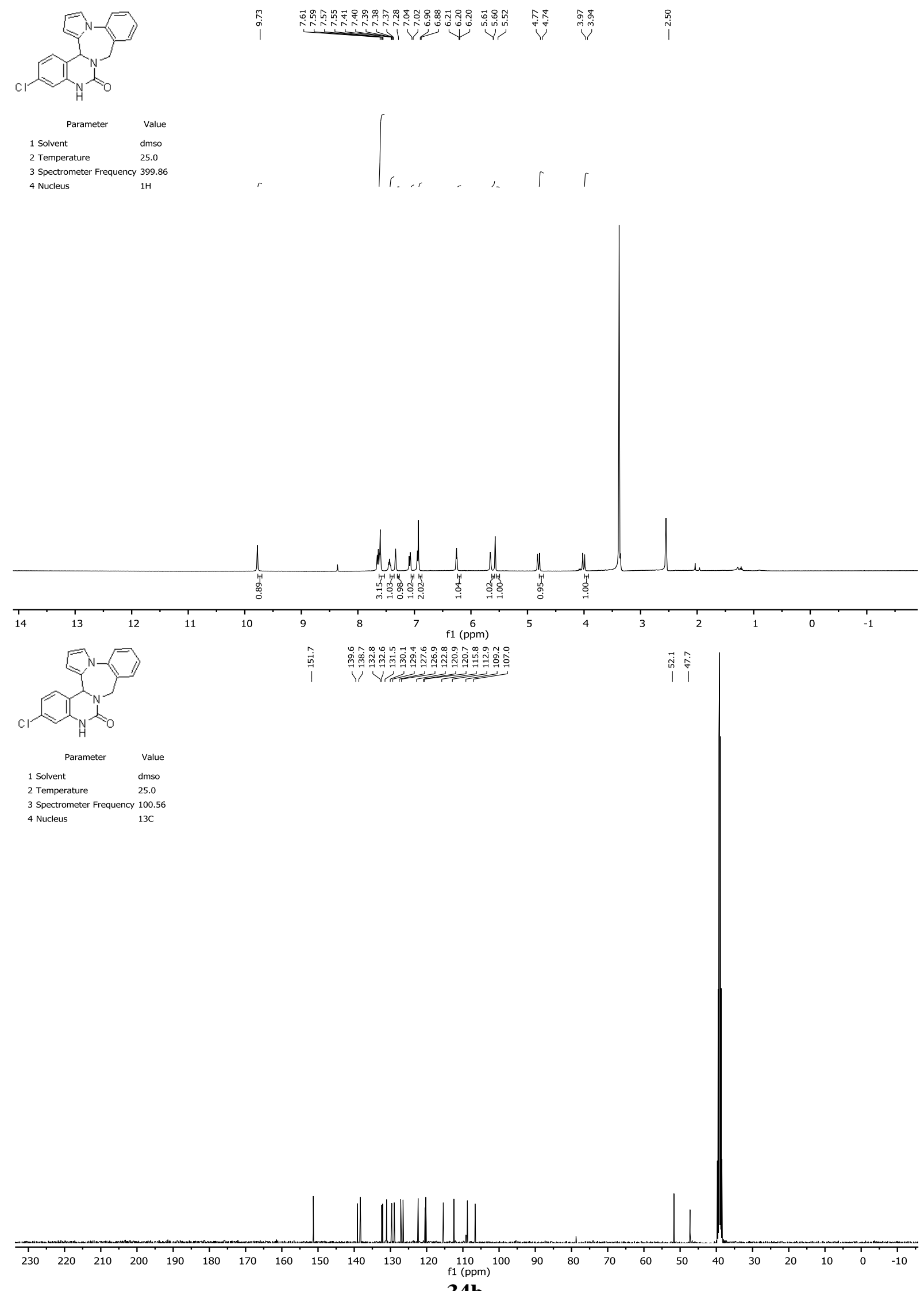

34b 

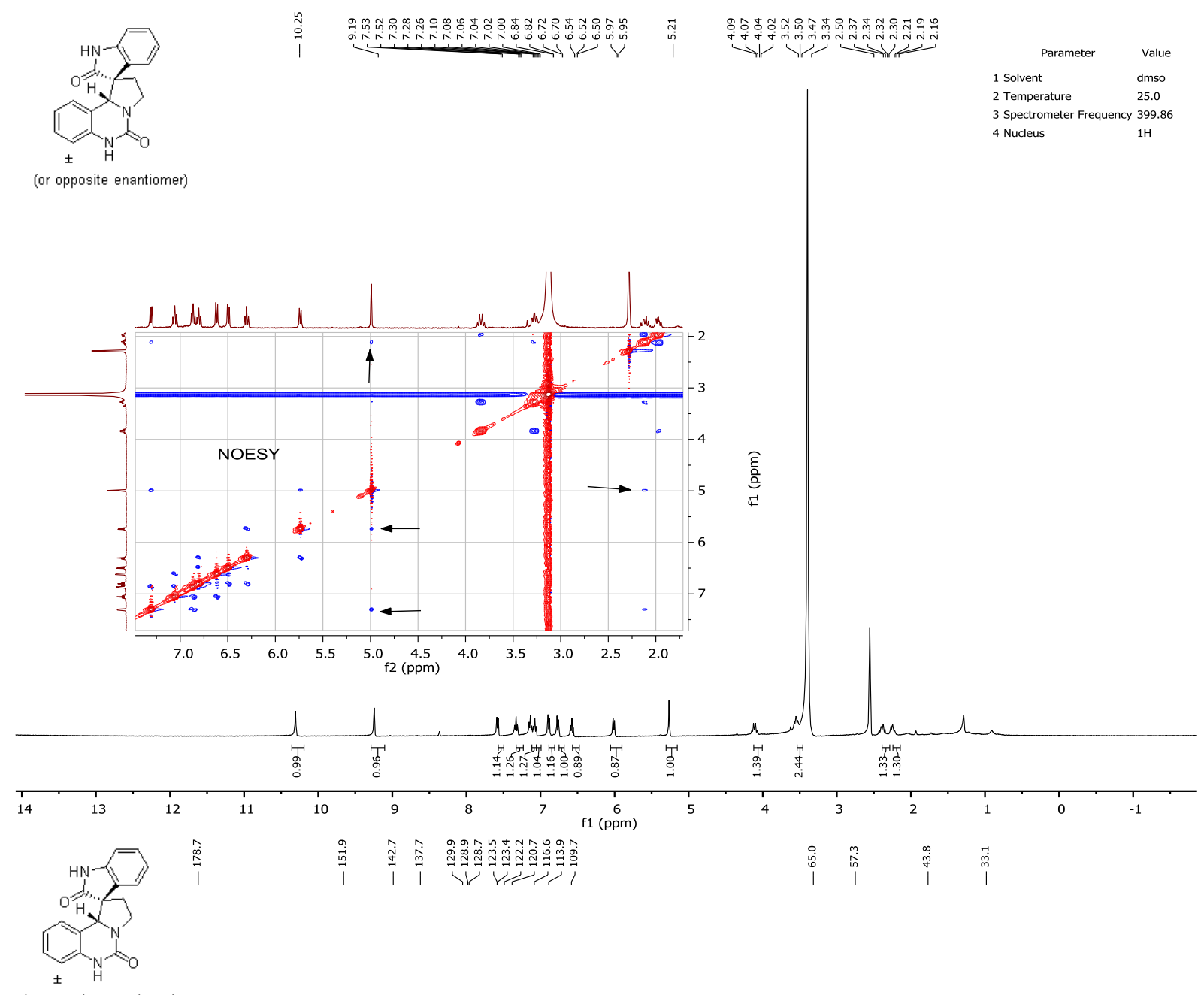

(or opposite enantiomer)
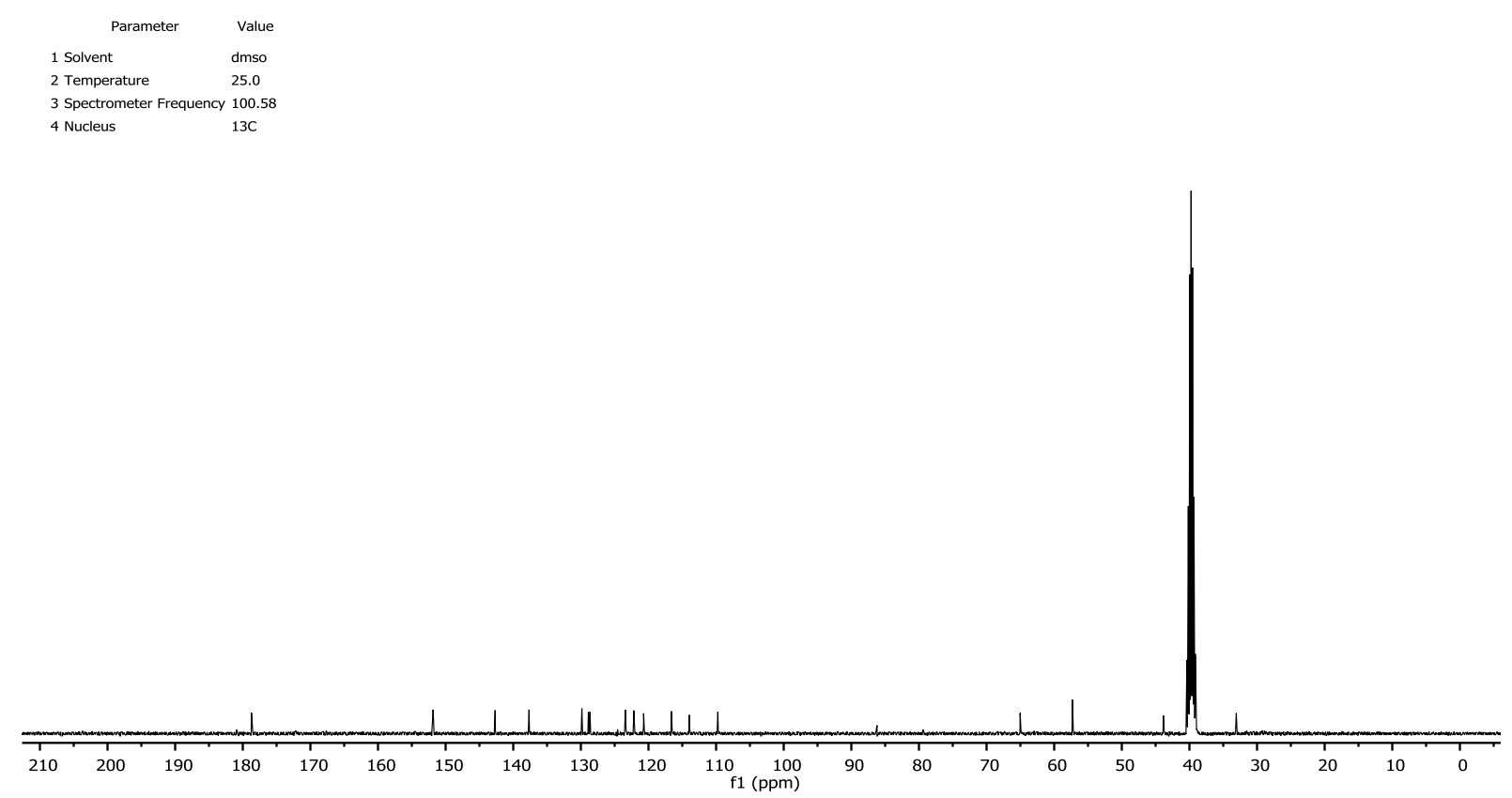

35a 

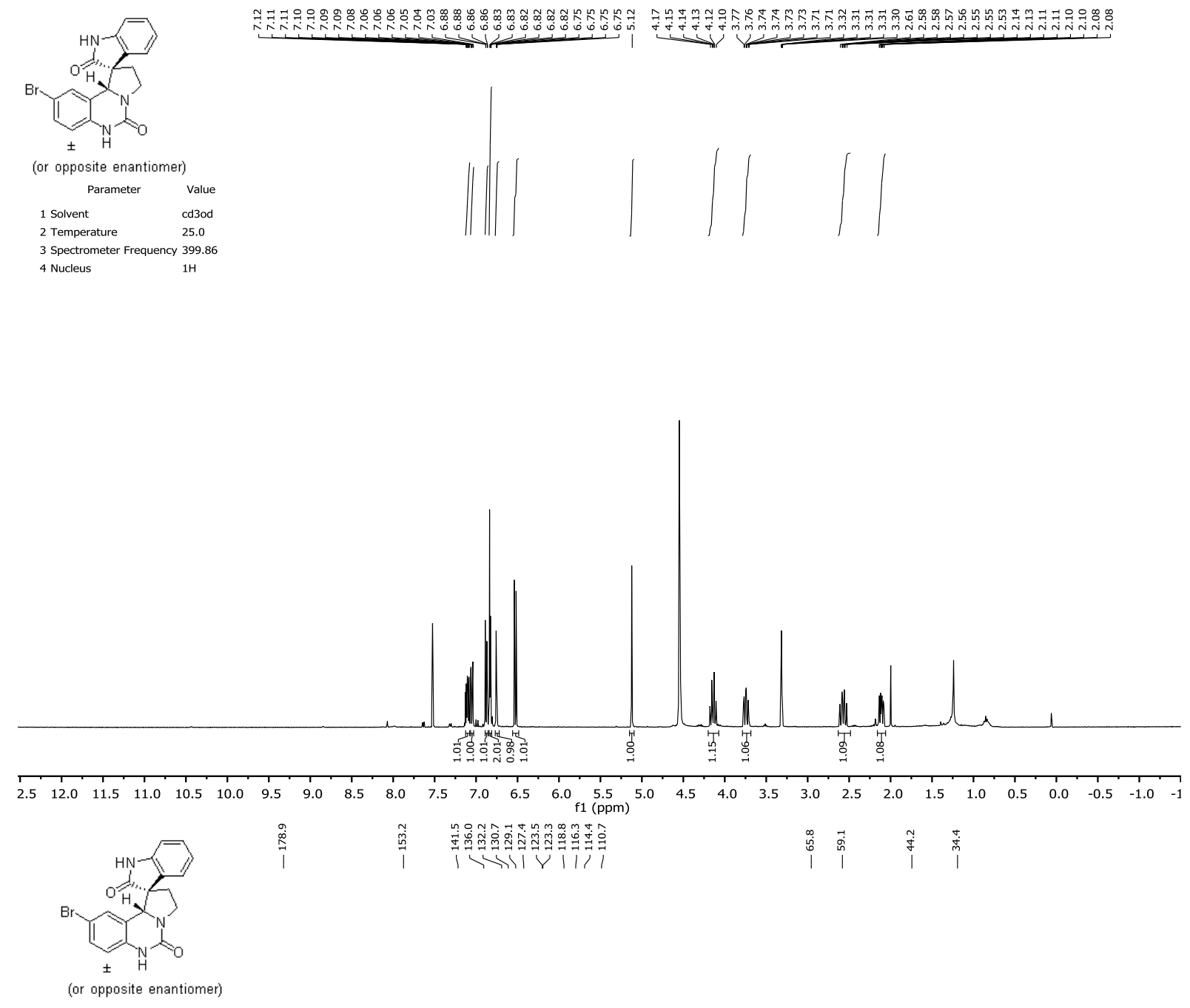

3 Spectrometer Frequency 100.56

4 Nucleus

$13 \mathrm{C}$

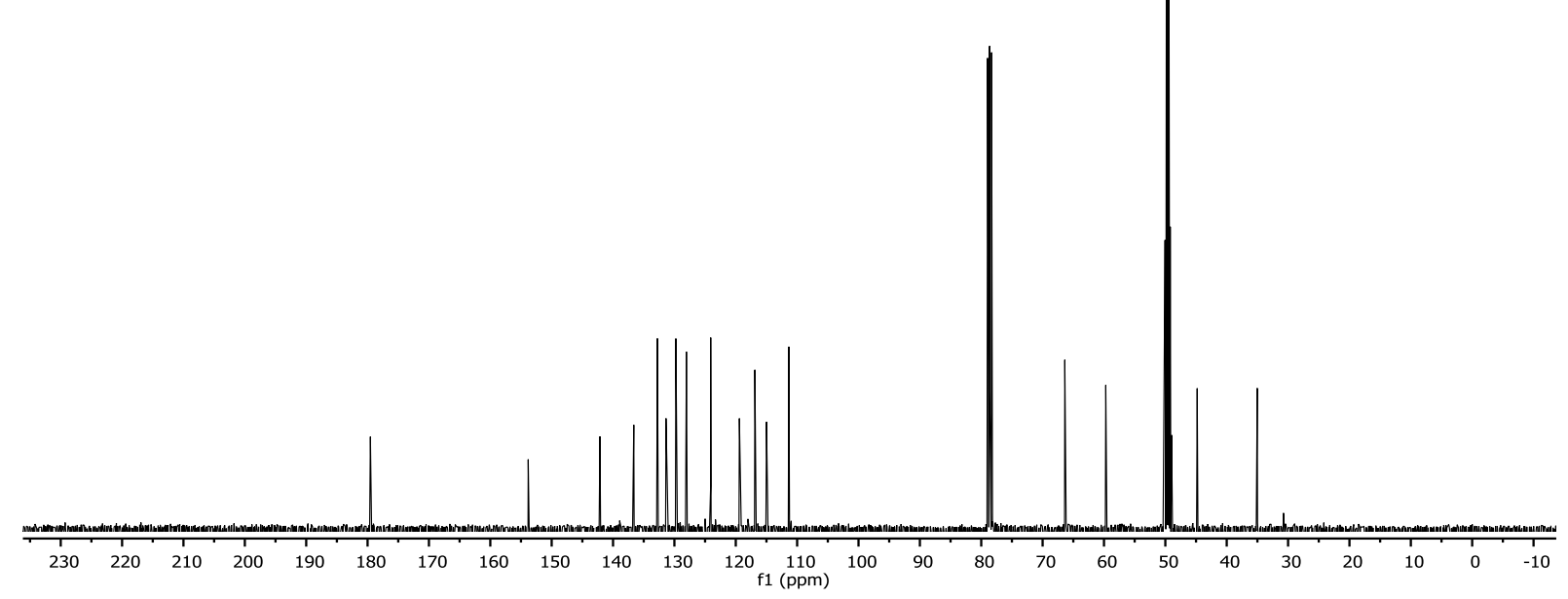
35b 

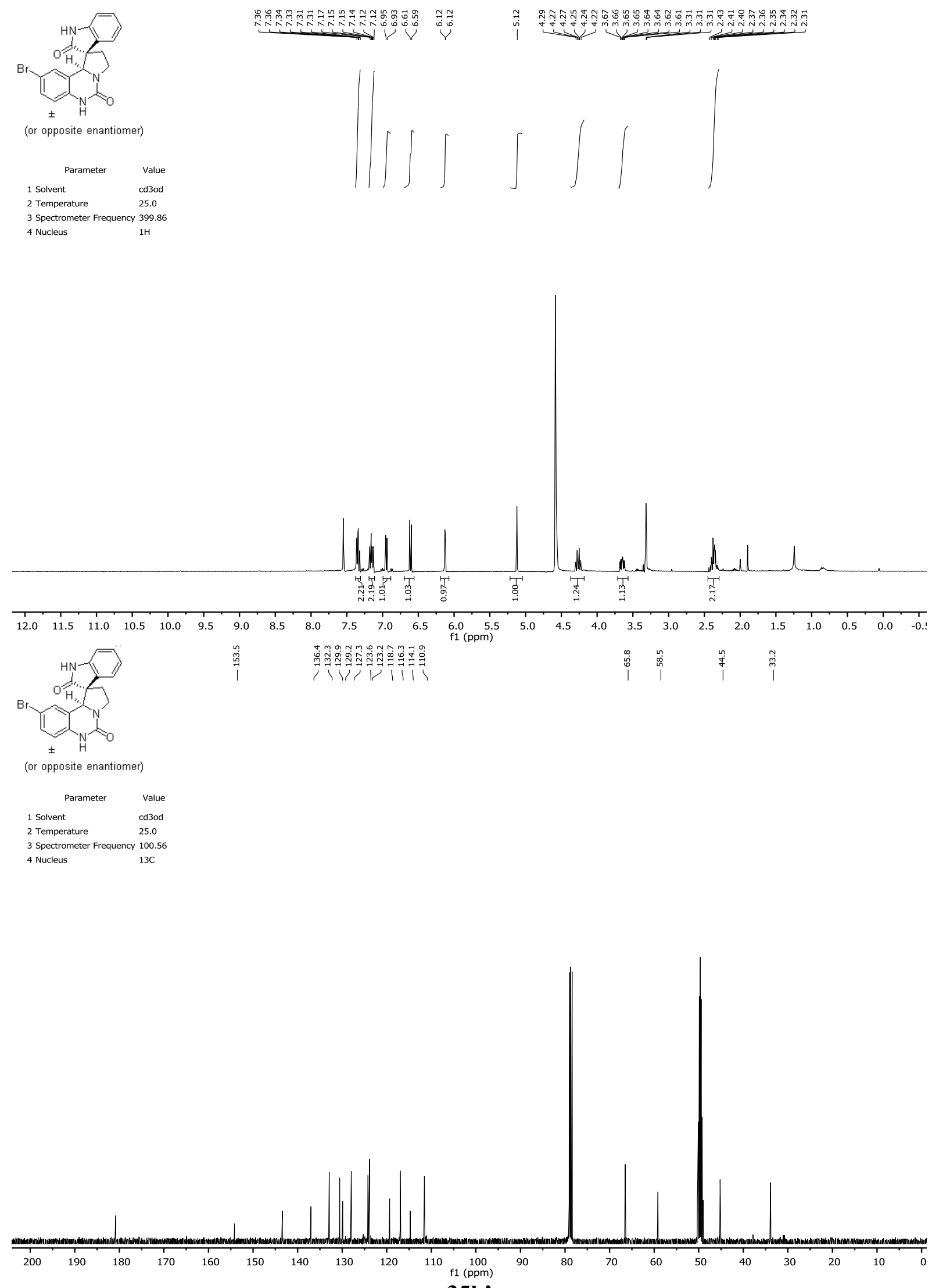

35b' 


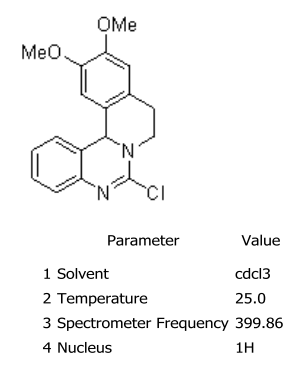

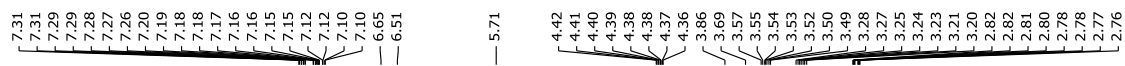
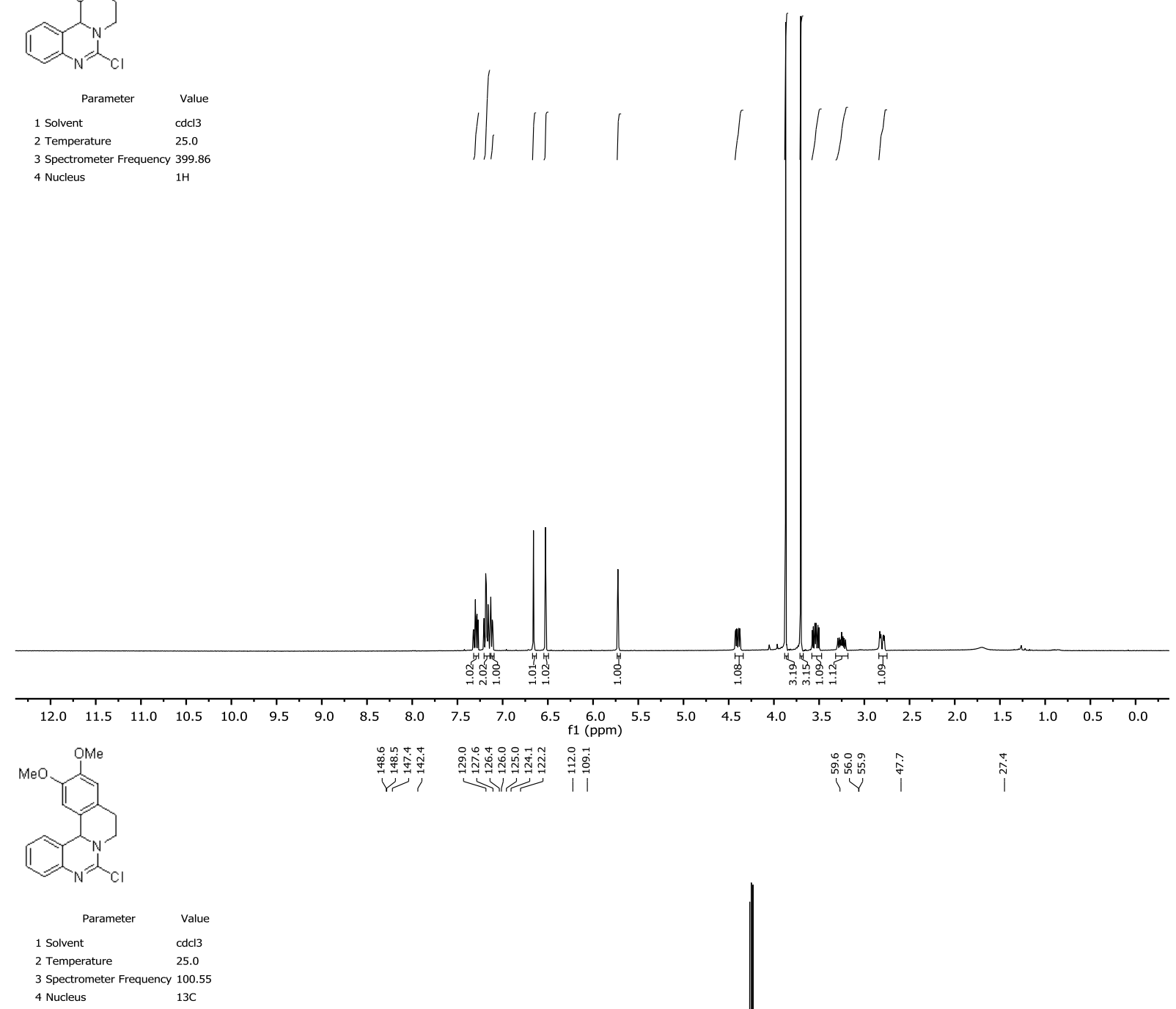

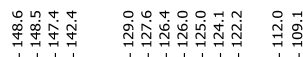

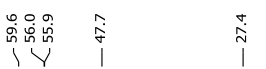

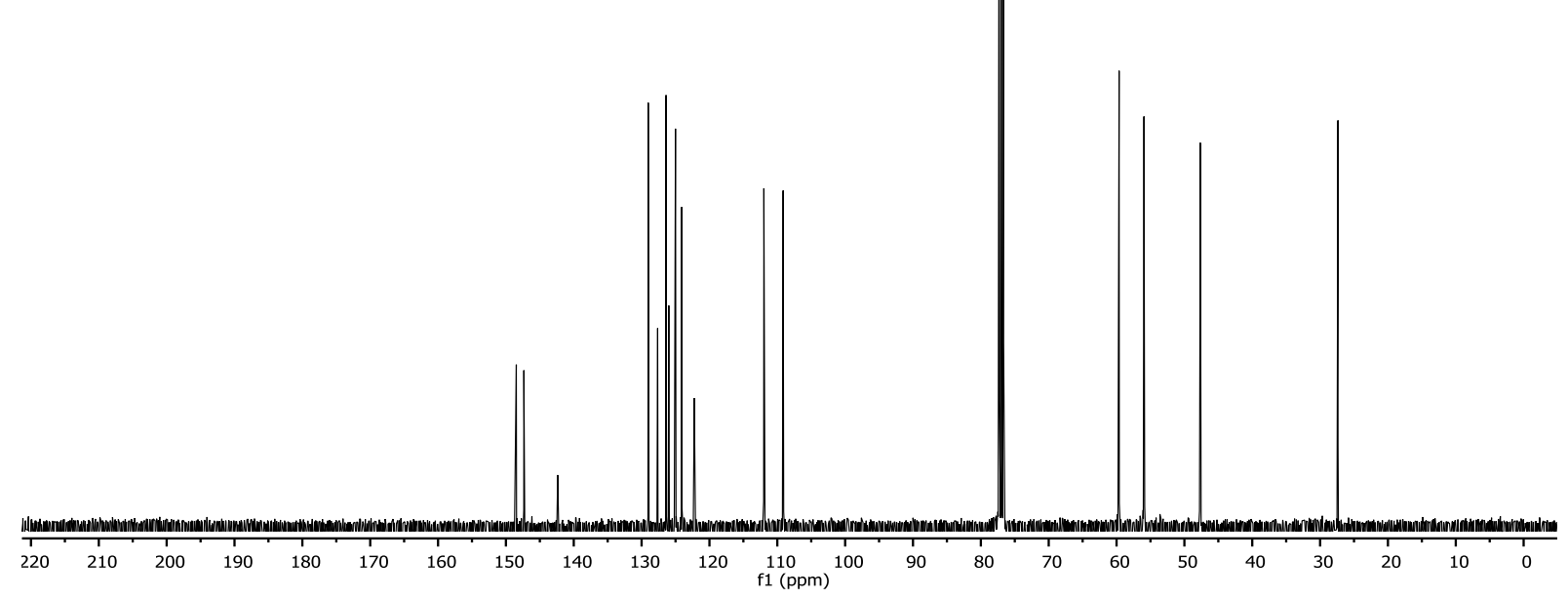

36a 


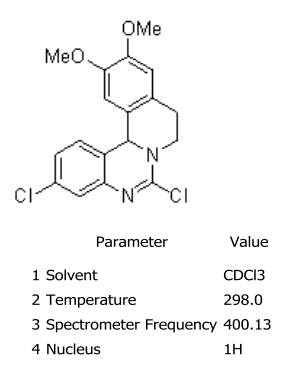

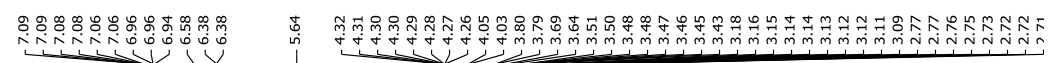
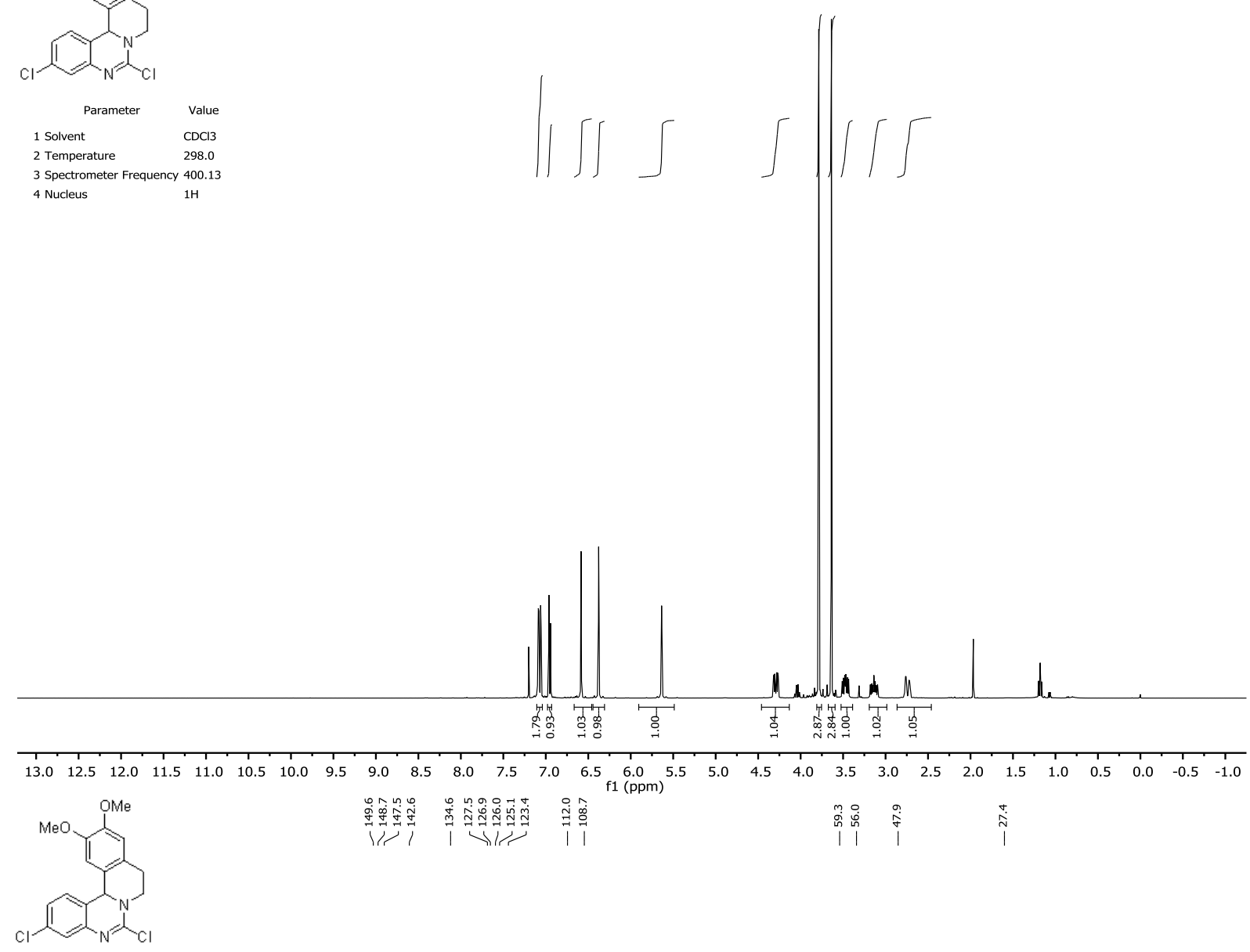

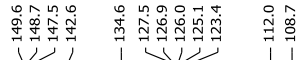

mån

\begin{tabular}{lc}
\multicolumn{1}{c}{ Parameter } & Value \\
1 Solvent & CDC13 \\
2 Temperature & 298.0 \\
3 Spectrometer Frequency & 100.62 \\
4 Nucleus & $13 \mathrm{C}$
\end{tabular}

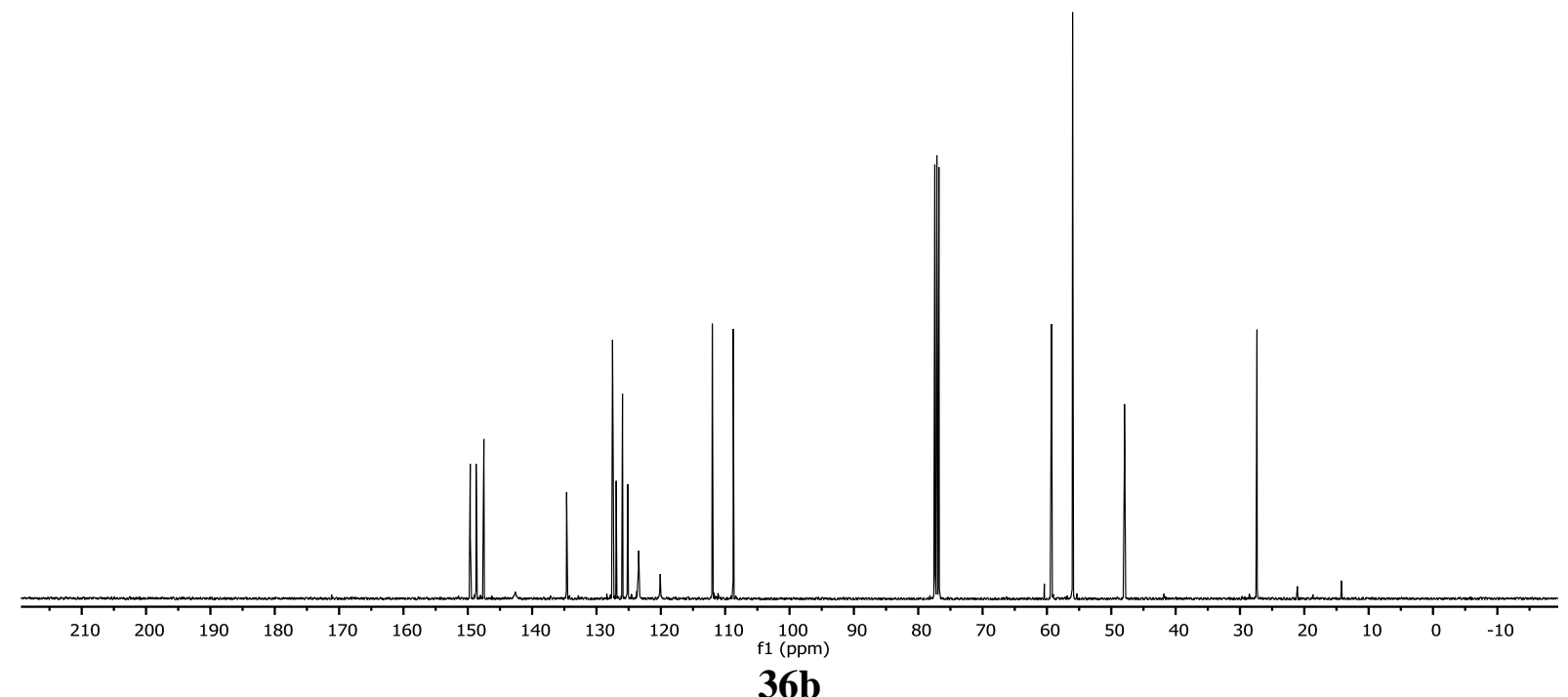



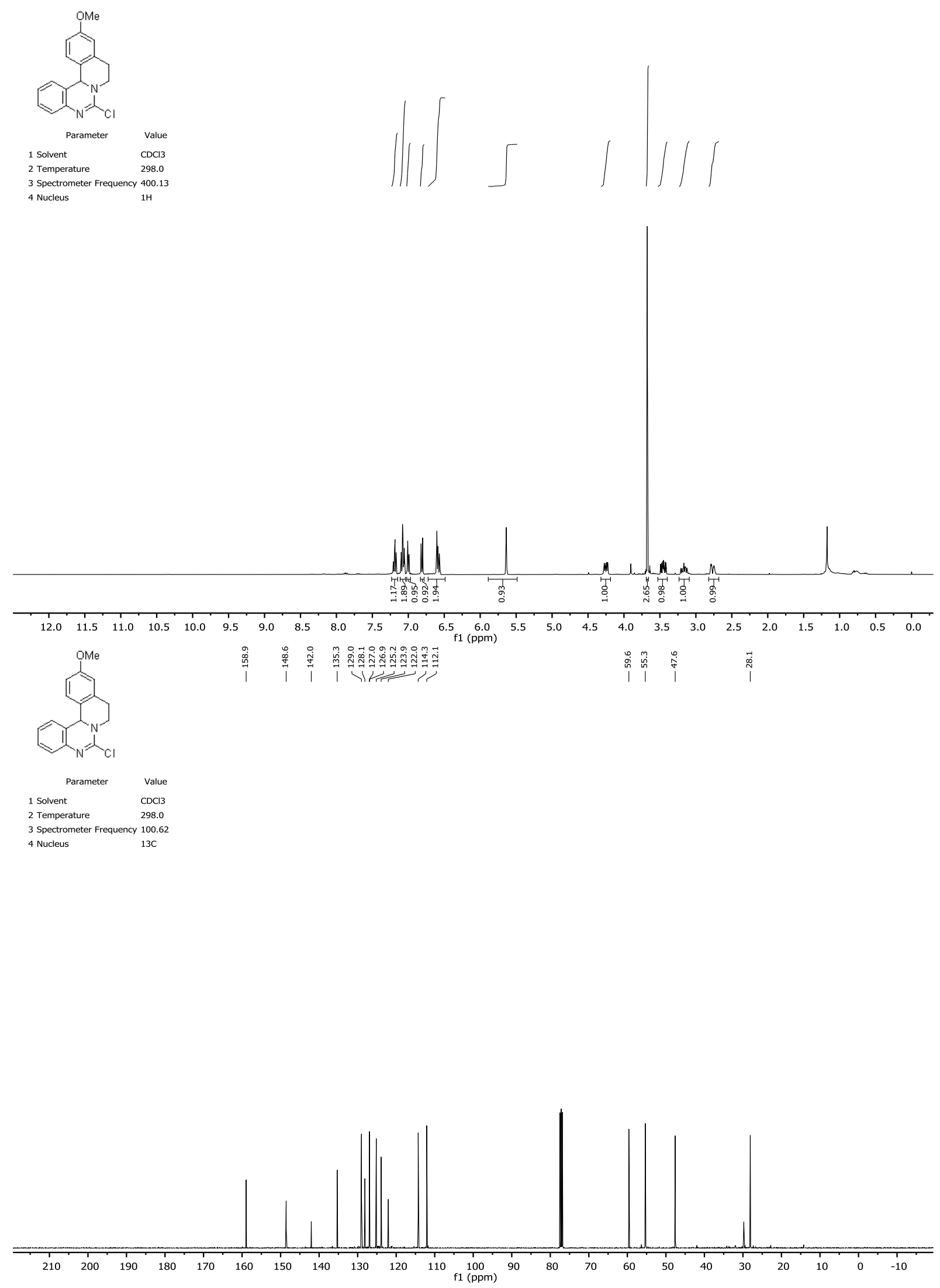

36c 

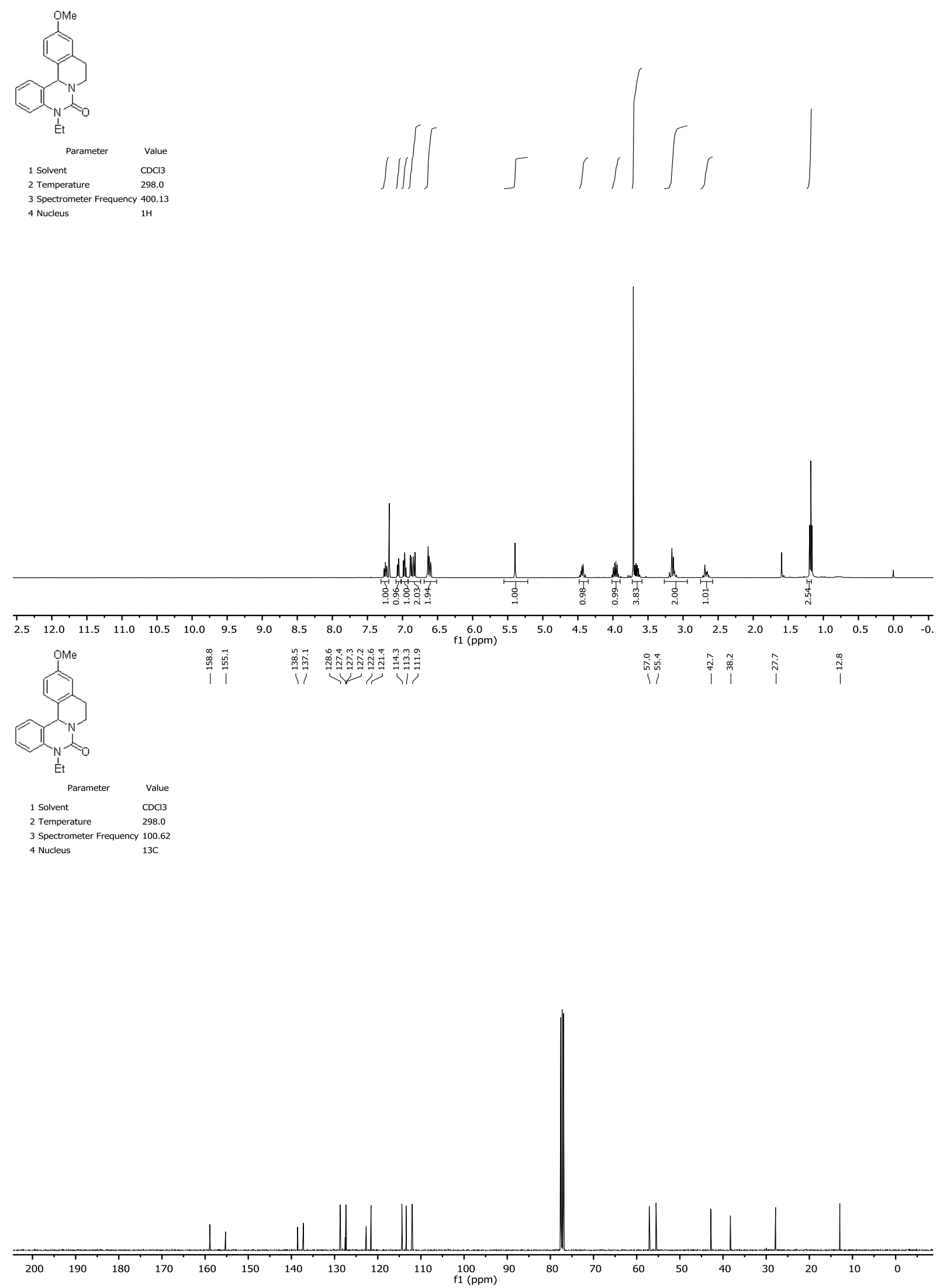

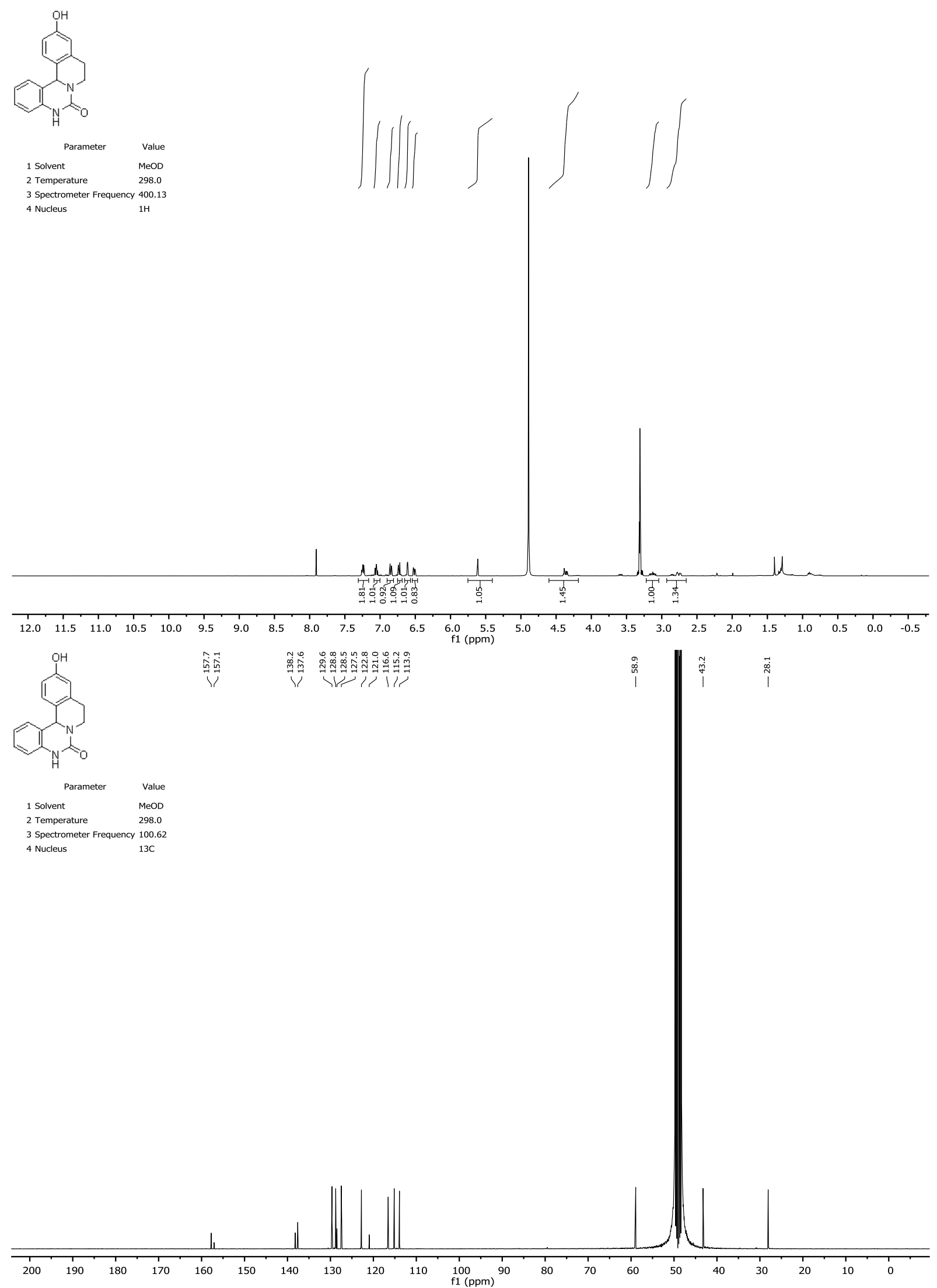


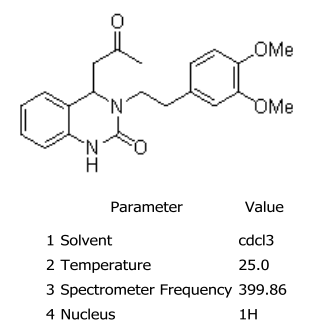

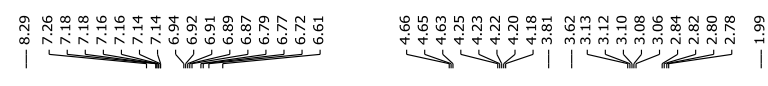

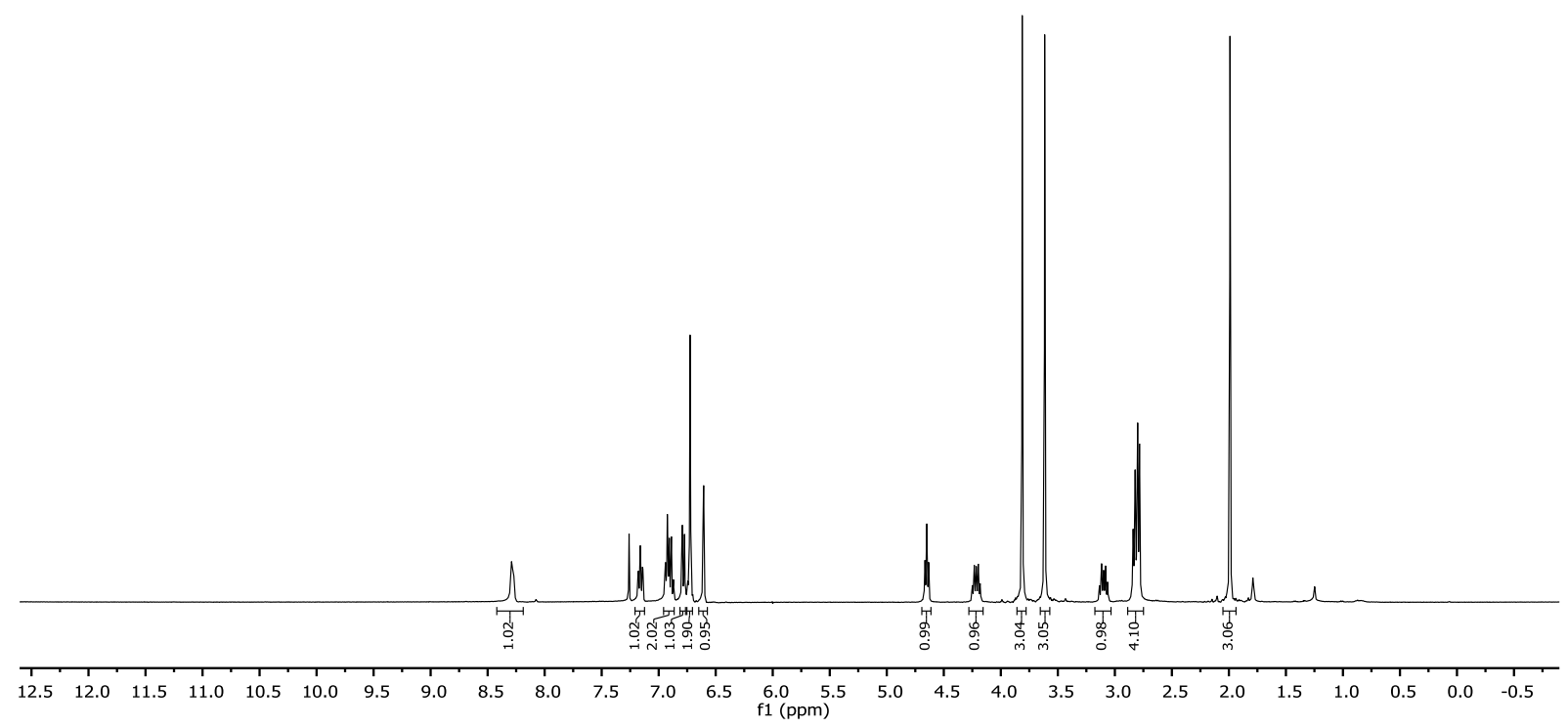

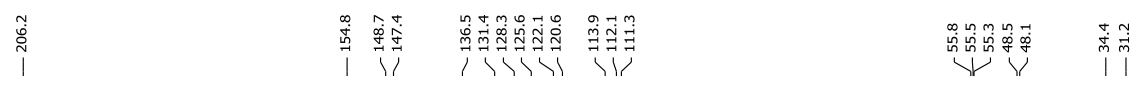
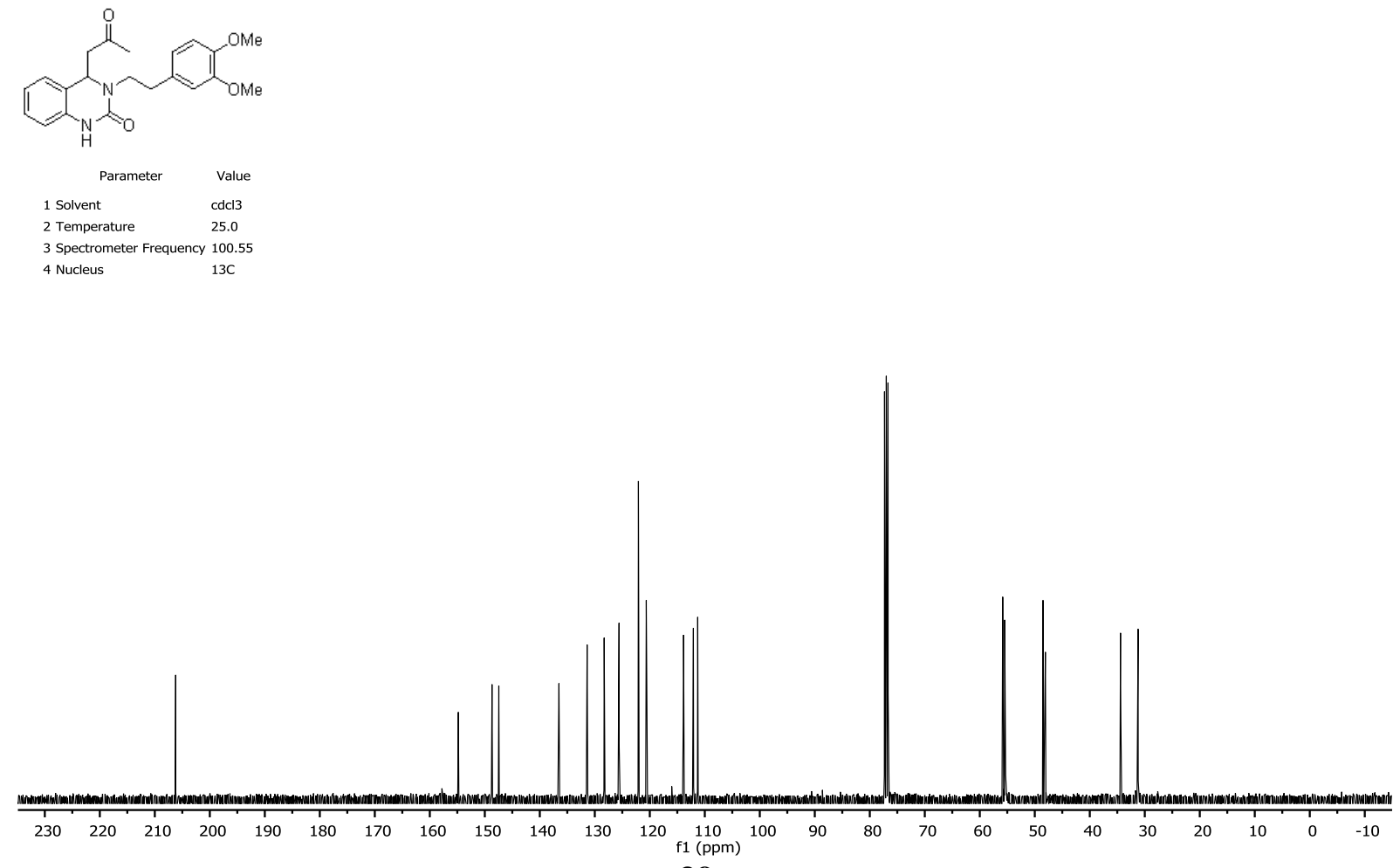

39a 

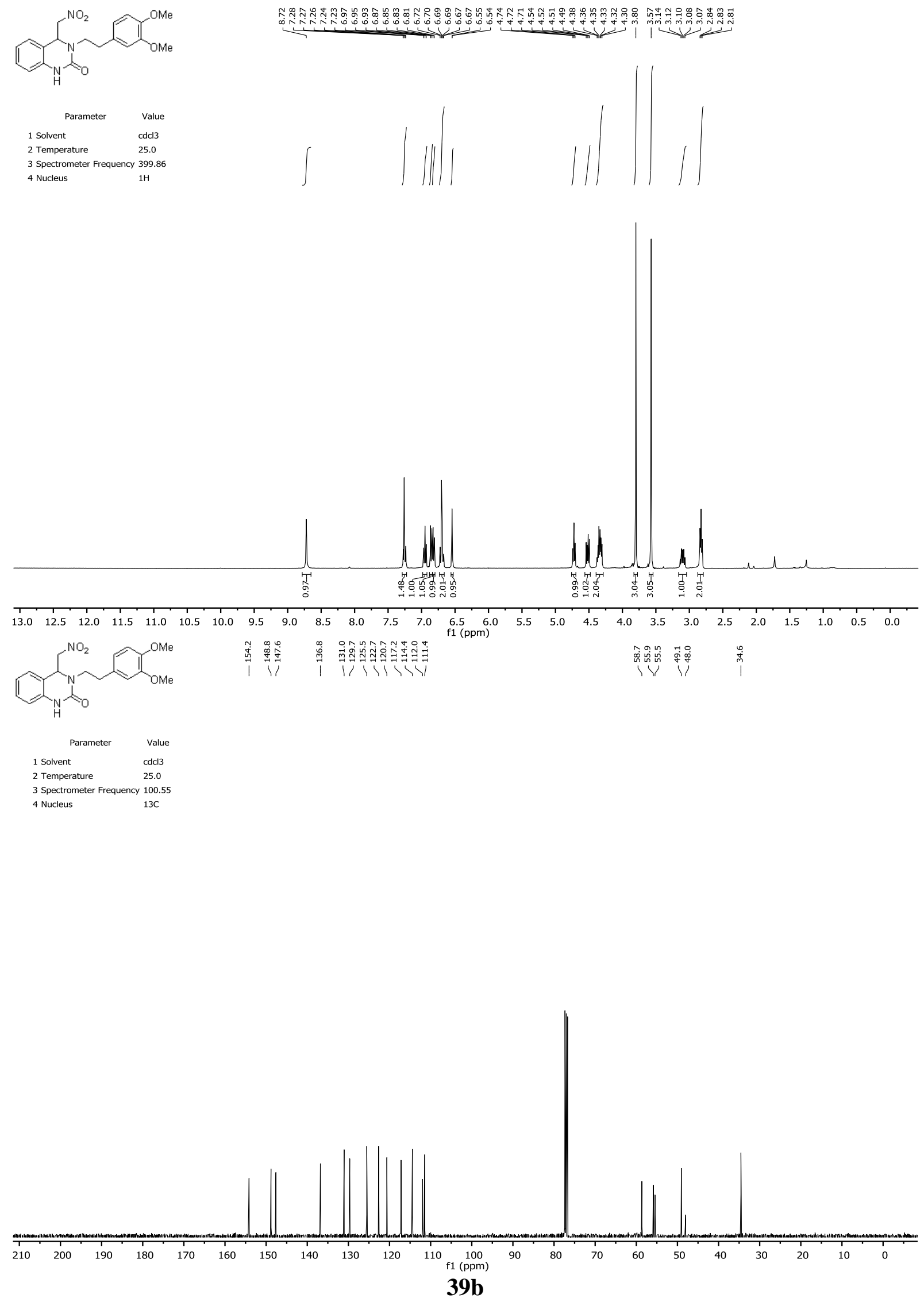

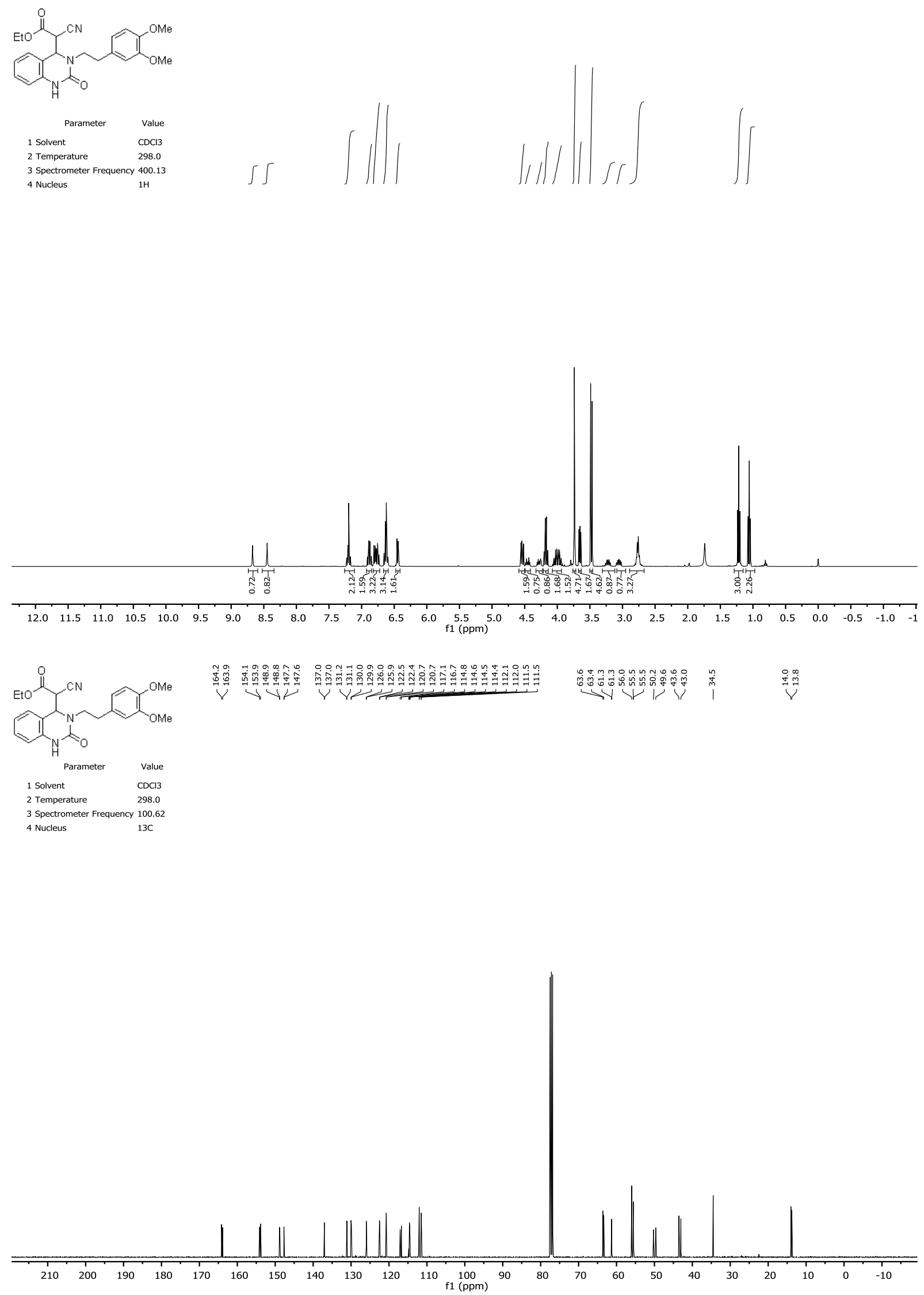

39c 